Purdue University

Purdue e-Pubs

Open Access Theses

Theses and Dissertations

Spring 2014

\title{
Experimental investigation of a forced response condition in a multistage compressor
}

William Louis Murray

Purdue University

Follow this and additional works at: https://docs.lib.purdue.edu/open_access_theses

Part of the Aerospace Engineering Commons, and the Mechanical Engineering Commons

\section{Recommended Citation}

Murray, William Louis, "Experimental investigation of a forced response condition in a multistage compressor" (2014). Open Access Theses. 735 .

https://docs.lib.purdue.edu/open_access_theses/735

This document has been made available through Purdue e-Pubs, a service of the Purdue University Libraries. Please contact epubs@purdue.edu for additional information. 


\section{PURDUE UNIVERSITY \\ GRADUATE SCHOOL \\ Thesis/Dissertation Acceptance}

This is to certify that the thesis/dissertation prepared

By William L. Murray III

Entitled

EXPERIMENTAL INVESTIGATION OF A FORCED RESPONSE CONDITION IN A

MULTISTAGE COMPRESSOR

For the degree of Master of Science in Aeronautics and Astronautics

Is approved by the final examining committee:

Dr. Nicole L. Key

Dr. Stephen Heister

Dr. Pavlos Vlachos

To the best of my knowledge and as understood by the student in the Thesis/Dissertation Agreement. Publication Delay, and Certification/Disclaimer (Graduate School Form 32), this thesis/dissertation adheres to the provisions of Purdue University's "Policy on Integrity in Research" and the use of copyrighted material.

Dr. Nicole L. Key

Approved by Major Professor(s):

Approved by: Wayne Chen $04 / 14 / 2014$ 

EXPERIMENTAL INVESTIGATION OF A FORCED RESPONSE CONDITION IN A MULTISTAGE COMPRESSOR

\author{
A Thesis \\ Submitted to the Faculty \\ of \\ Purdue University \\ by \\ William Louis Murray III \\ In Partial Fulfillment of the \\ Requirements for the Degree \\ of \\ Master of Science in Aeronautics and Astronautics
}

May 2014

Purdue University

West Lafayette, Indiana 
To my fiancée and the love of my life, Yekaterina. 


\section{ACKNOWLEDGEMENTS}

I would like to thank, firstly, Dr. Nicole Key for her guidance with my research, for always doing her best to be available when I needed assistance, and for providing such a wonderful experience at the Purdue Compressor Lab that has taught me so much about a field I knew so little about when I started two years ago. I would also like to thank Natalie Smith and Reid Berdanier for their immense support in setting up experiments, help with running codes, and moral guidance while here at Purdue, and I would not have done as much as I did if it were not for them. Lou Fangyuan has been a wonderful partner to have on the Honeywell work, and together we have accomplished an immense amount of work, and he has helped me tremendously. Nick, Evan, Patrick, Dave, Bryce, Matt. John Fabian, Allison, Geoff, and Dr. John Brossman have been wonderful friends and coworkers and I have enjoyed every day I have had the privilege to work alongside them. Lastly, if it wasn't

for the suggestions of David Reese and Jordan Forness, I wouldn't have made the trip from USC to Purdue to continue my studies in the first place.

Most importantly, I would like to thank the entire GUIde IV consortium for their funding, help, and support throughout this process, in particular Rakesh Srivastava and Roy Fulayter for their support and guidance. I also would like to thank Honeywell for their funding and support throughout my entire thesis. 


\section{TABLE OF CONTENTS}

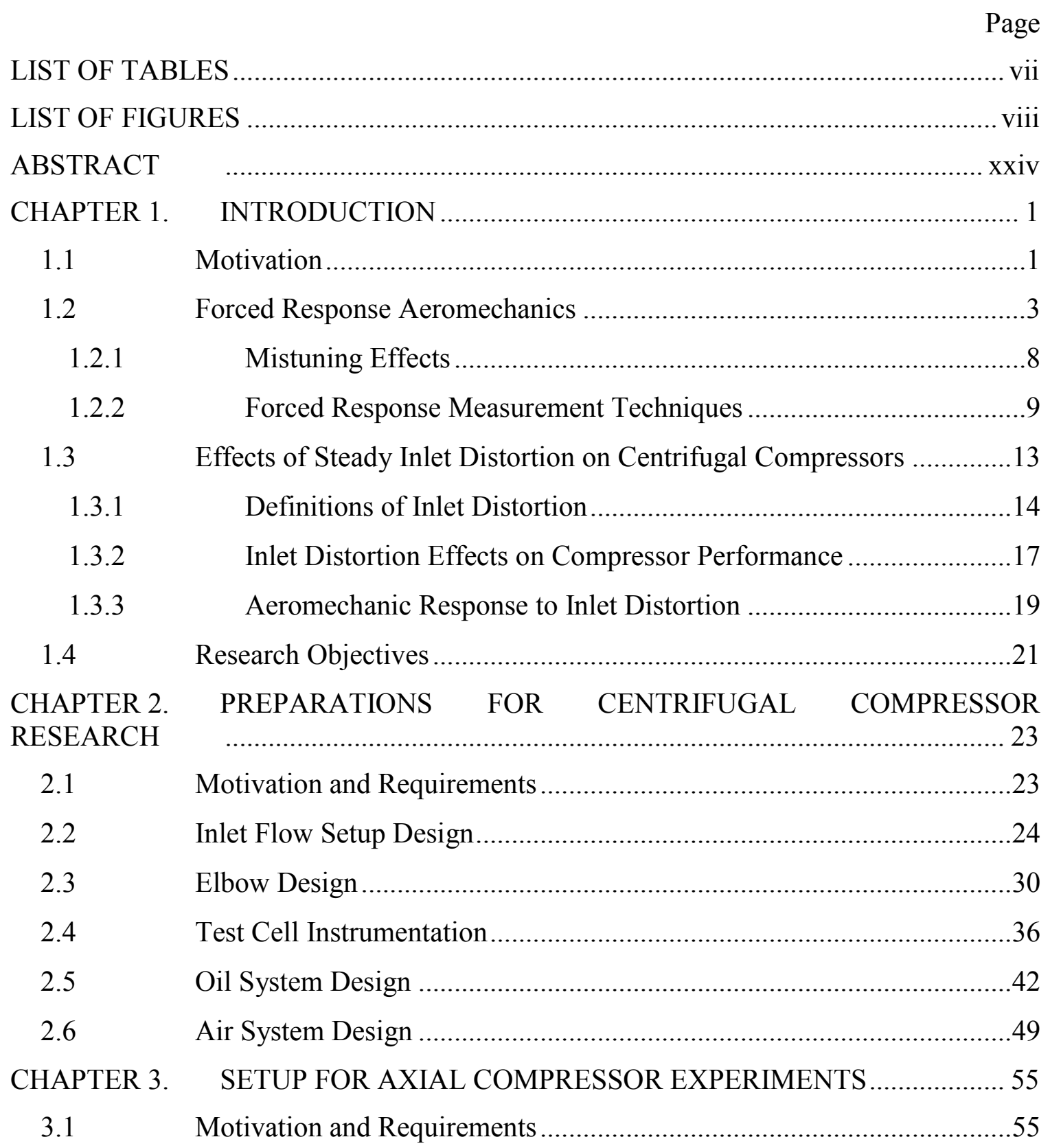




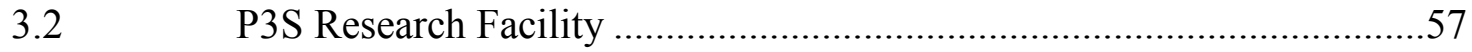

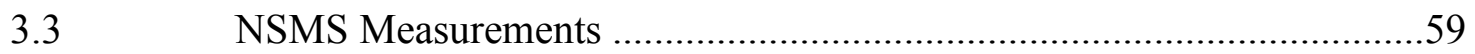

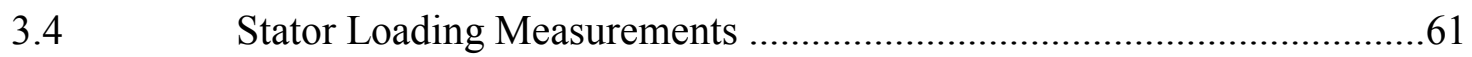

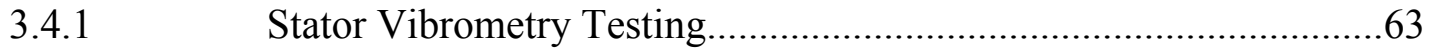

3.4.2 Stator Kulite Pressure Measurements ................................................66

3.5 Casing Pressure Measurements..........................................................

3.6 Thermal Anemometry Measurement ……….......................................

CHAPTER 4. CHARACTERIZTION OF FORCING FUNCTIONS \& ROTOR

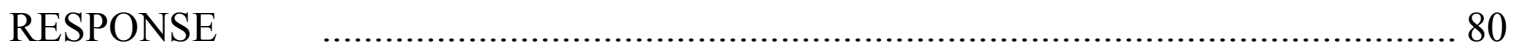

$4.1 \quad$ Compressor Operating Conditions ........................................................

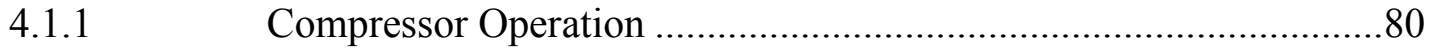

4.1.2 Steady Performance ……………………....................................

4.2 Forcing Functions for 44 EO R2 1T Resonance ....................................86

4.2.1 Vortical Forcing Function ...............................................................

4.2.2 Potential Forcing Function...............................................................93

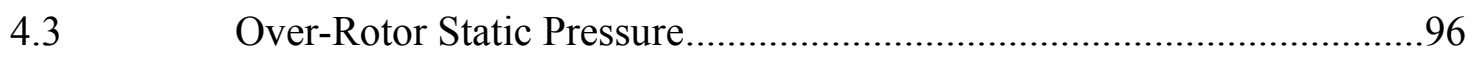

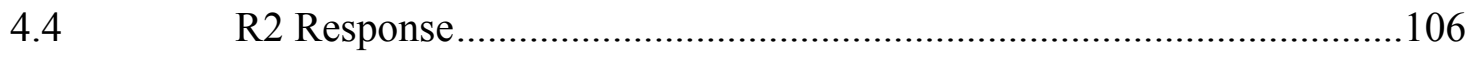

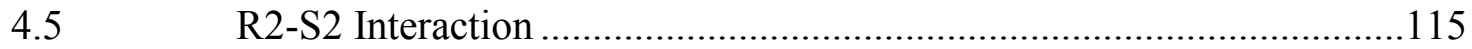

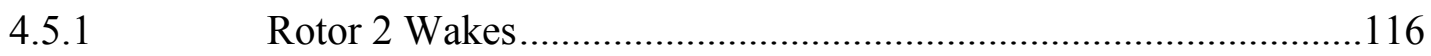

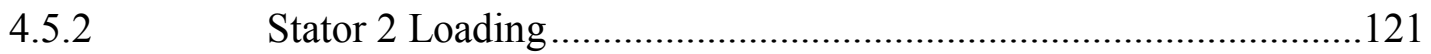

CHAPTER 5. EVIDENCE OF R2 VIBRATION IN UNSTEADY FLOW FIELD

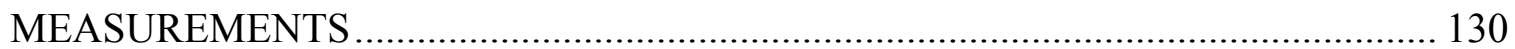

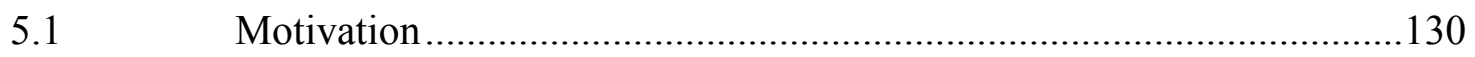

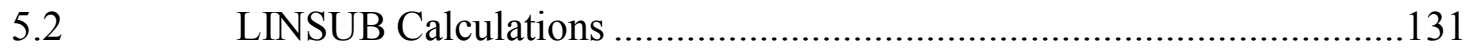

5.3 Data Processing Strategy to Detect Blade Vibration ..............................134

5.3.1 Reconciling Revolutions of Transient Data ....................................135

5.3.2 PXI Lag Offset .....................................................................

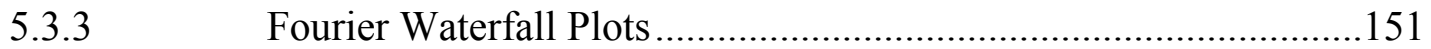

5.3.4 Cross-Correlation Techniques........................................................171

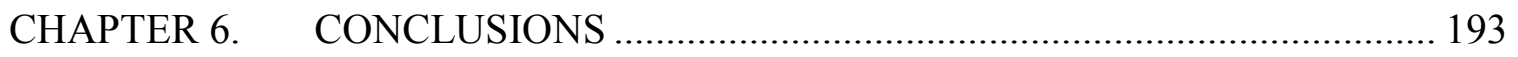




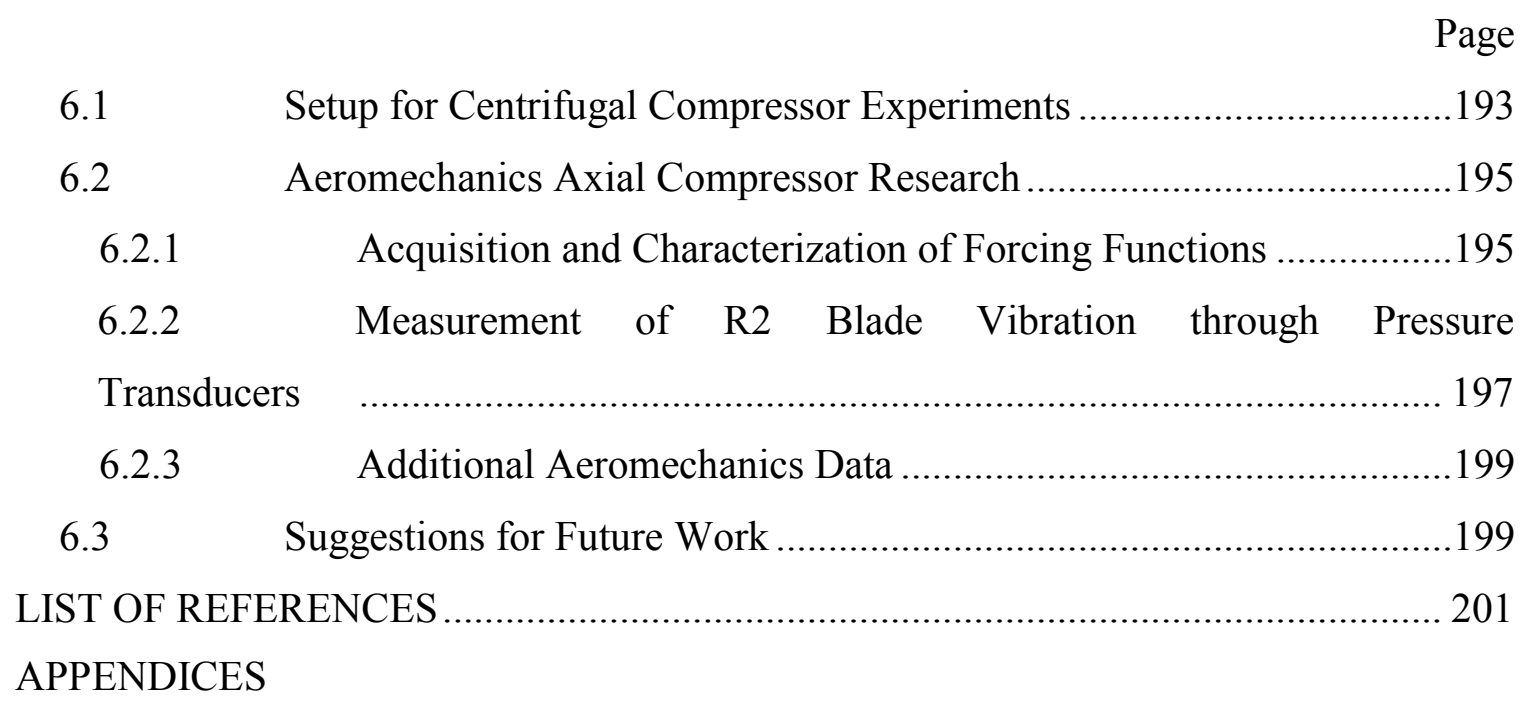

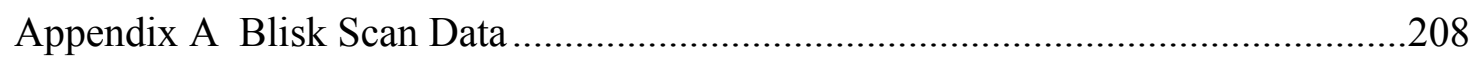

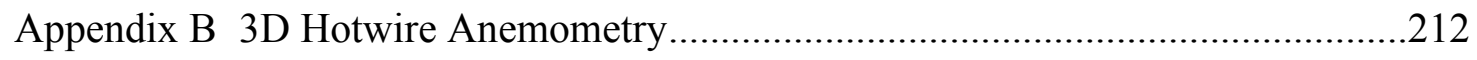

Appendix C Kulite Windowing Techniques ........................................................219

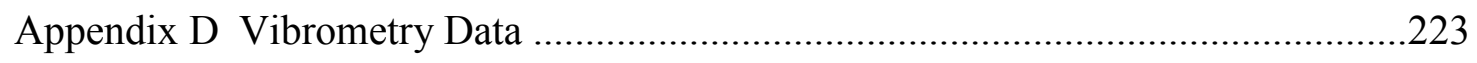

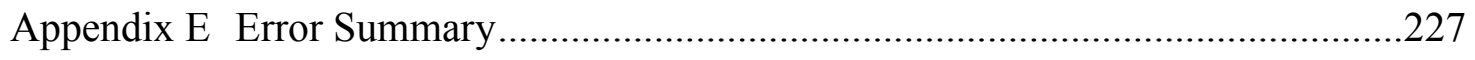




\section{LIST OF TABLES}

Table

Page

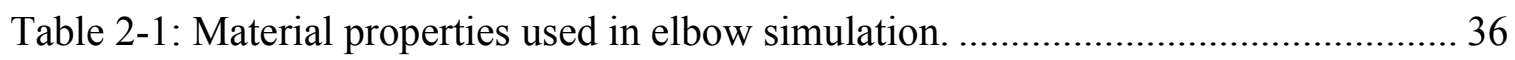

Table 2-2: Margins in Lucite and Stainless from overpressure simulation. .................... 36

Table 2-3: List of major data acquisition equipment purchased for Honeywell research. 37

Table 2-4: Honeywell oil requirements for sections of SSCC rig. …........................... 47

Table 2-5: Venturi design for Diffuser Match Bleed (1), Diffuser Back Face Bleed (2). 52

Table 2-6: Air flow requirements for all air regulators in Honeywell air system............. 54

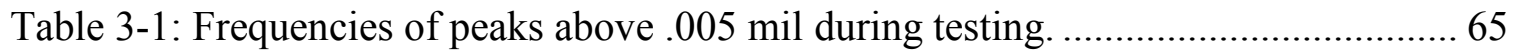

Table 4-1: Summary of R2 blade responses through 4 different runs at 2 loadings....... 113

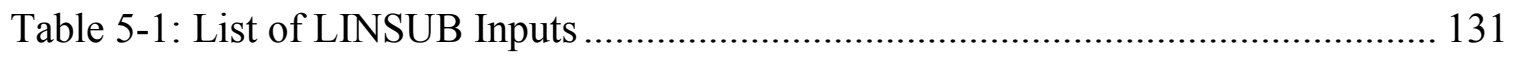

Table 5-2: File name, sample rate, and time of test files used to determine lag offset... 145

Table 5-3: Time offset per sample for different PXIE sample rates........................... 149

Table 6-1: Select points from calibration of Dantec Type 55-14677501 on 2/21/2013 . 214

Table 6-2: Select points from calibration of Dantec Type 55-1467750 on 3/10/2013 ... 215

Table 6-3: NSMS P-P deflection uncertainty and LSMF model fit number per R2

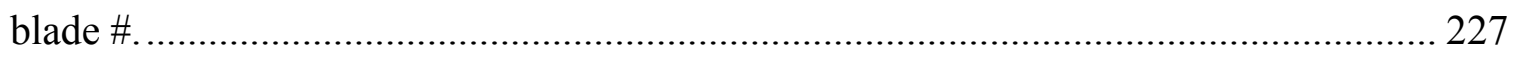




\section{LIST OF FIGURES}

Figure

Page

Figure 1.1: Campbell diagram showing 4 EOs and three modes, Richards et al. (2012).. 4

Figure 1.2: Design of radial inlet for a centrifugal compressor with IGVs, Kim and Koch (2004).

Figure 1.3: Inlet total pressure distortion, normalized by upstream pressure rakes, Zemp, et al. (2010). 16

Figure 2.1: Proposed cell layout with ABB motor, gearbox, compressor, and APU inlet. 24

Figure 2.2: Engineering drawing of Schutte and Koerting Air Ejector. 25

Figure 2.3: Ejector Flow Schematic. 27

Figure 2.4: Required $C v$ for different pressures to maintain ejector motive flow. 28

Figure 2.5: Test cell with Ejector setup, main dimension in inches. 29

Figure 2.6: APU Inlet installed in test cell in orientation for Ejector flow with LDV instrumentation access. 29

Figure 2.7: Outdoor ejector installation model, showing motive air plumbing. 30

Figure 2.8: Section view of redesigned elbow, illustrating Lucite SAR windows for LDV. 32

Figure 2.9: The final elbow design, with .062" thick aluminum sheet walls and LUCITE SAR acrylic windows for LDV optical access. 32 
Figure

Figure 2.10: Static structural stresses in metallic components. Yield allowable is 32.3

ksi. 34

Figure 2.11: Static structural stresses as seen by top of acrylic window, where max stress occurs. 35

Figure 2.12: Part deformations, maximum seen in window of 0.139 ” at max pressure... 35

Figure 2.13: Tower with instrumentation and ABB motor control pedestal. 40

Figure 2.14: View of Honeywell control station. 41

Figure 2.15: High speed data acquisition and amplification hardware 41

Figure 2.16: 3D SolidWorks model of outdoor heat exchanger enclosure. 44

Figure 2.17: Oil and air system legend. 48

Figure 2.18: Honeywell oil system. 49

Figure 2.19: Honeywell air system. 53

Figure 3.1: Campbell diagram showing R2 1T, where GUIde IV is centered around. .... 56

Figure 3.2: Purdue 3 stage axial compressor research facility. 58

Figure 3.3: Cross section of Purdue 3-stage axial compressor, showing relevant station numbers. 59

Figure 3.4: NSMS Probe circumferential placement in casing, forward facing aft......... 60

Figure 3.5: 3D model of stator before Kulite installation. 62

Figure 3.6: Stator 2 EDM cut into three sections for stator Kulite instrumentation......... 62 Figure 3.7: Depiction of stator blade with Kulite sensors installed at 10\%, 20\%, 30\% and $40 \%$ chord. 62

Figure 3.8: Vibrometry testing of stator half ring with piezo-electric driver at WPAFB. 64 
Figure

Figure 3.9: Vibrometry testing of stator 2 insert. Only case where piezo driver supported

the stator section being tested. 64

Figure 3.10: Stator section upon arrival from Kulite. 66

Figure 3.11: Close up of stator section showing RTV on backside of installed Kulites. . 66 Figure 3.12: Stator installed in cap of calibration chamber, instrumentation cables fed out of cap. 67

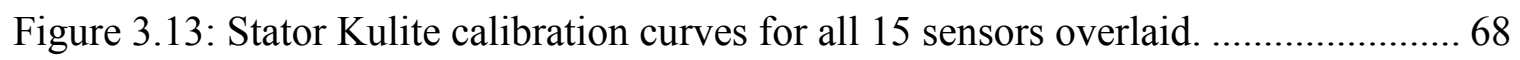

Figure 3.14: Calibration chamber with snap ring and removable bulkhead................... 69

Figure 3.15: Kulites mounted in window block of baseline compressor casing. ............ 70

Figure 3.16: Sensor positions relative to R2 blade, with select sensors labeled, CL 1 stator

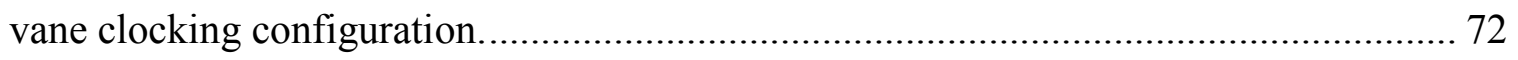

Figure 3.17: Calibration of all 25 sensors installed in casing Kulite window, overlaid... 72 Figure 3.18: Purdue Hotwire Calibration Facility (Morrison, 2013)............................. 73

Figure 3.19: Velocity calibration of TSI cross flow film probe. ................................. 74

Figure 3.20: Yaw calibration of TSI cross flow film probe...................................... 74

Figure 3.21: Density calibration for TSI 1247A-10 Cross Film probe, Wire 1............... 76

Figure 3.22: Density calibration for TSI 1247A-10 Cross Film probe, Wire 2 ............... 76

Figure 3.23: Change in density sensitivity as a function of velocity, Wire $1 \ldots \ldots \ldots \ldots \ldots \ldots . . . .78$

Figure 3.24: Change in density sensitivity as a function of velocity, Wire 2................ 78

Figure 3.25: Illustration of angle decomposition used to resolve velocity components for both hotwire axial locations. Flow from right to left, as seen by probe. 79 
Figure

Figure 4.1: NSMS data showing RPM band and relative amplitude of R1, R2, and R3 1T

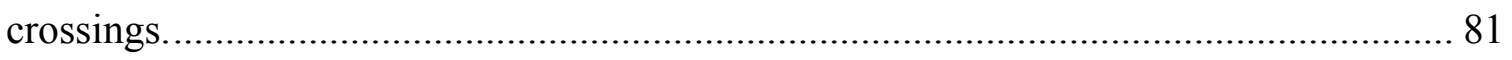

Figure 4.2: Compressor map showing regions of data acquired during aeromechanics

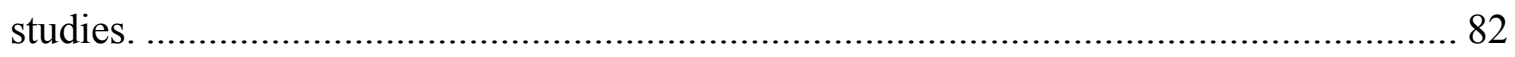

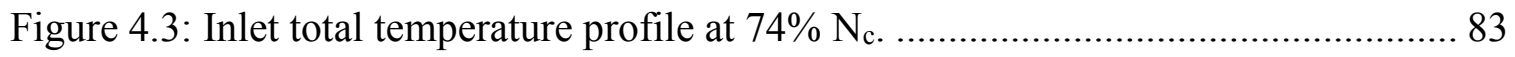

Figure 4.4: Casing surface temp as a function of axial position at $74 \% \mathrm{~N}_{\mathrm{c}} \ldots \ldots \ldots \ldots \ldots \ldots . . . . . . . . .83$

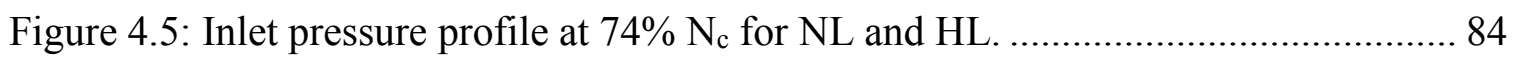

Figure 4.6: Steady total pressure measurements at three axial locations........................ 85

Figure 4.7: Steady total temperature measurements at three axial locations................... 85

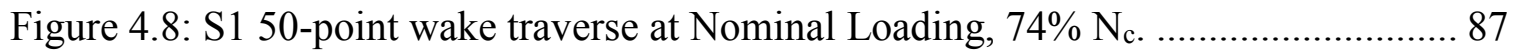

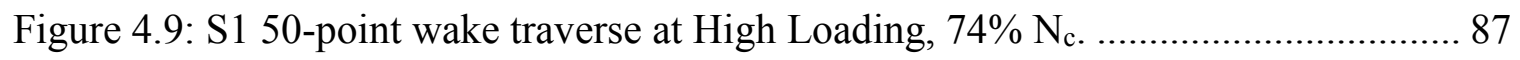

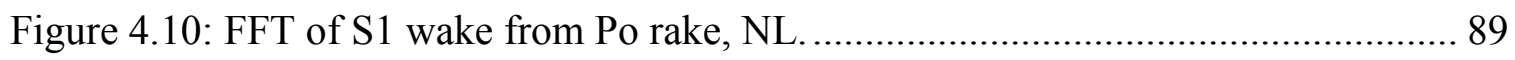

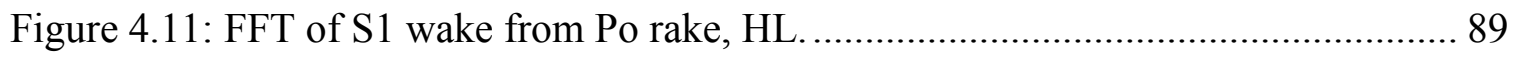

Figure 4.12: 40 point hotwire traverse of absolute velocity at S1 Exit, NL.................. 90

Figure 4.13: 40 point hotwire traverse of absolute velocity at S1 Exit, HL. .................. 91

Figure 4.14: FFT of S1 wake from hotwire traverse, NL...................................... 92

Figure 4.15: FFT of S1 wake from hotwire traverse, HL........................................ 93

Figure 4.16: Static pressure field downstream of Rotor 2 for NL and HL................... 94

Figure 4.17: Calculated $\alpha$ from a 20 point hotwire traverse of R2 exit, NL. ................ 95

Figure 4.18: Calculated $\alpha$ from a 20 point hotwire traverse of $\mathrm{R} 2$ exit, HL. ................. 95

Figure 4.19: Casing Kulite arrangement over Rotor 2 ........................................... 96 
Figure $\quad$ Page

Figure 4.20: Contour plot of time averaged casing Kulite pressures at 4100 RPM, flow from left to right, rotor rotation from top to bottom. (a) NL, (b) HL. 97

Figure 4.21: Standard deviation of mean pressure signal for casing Kulites at 4,100 RPM.

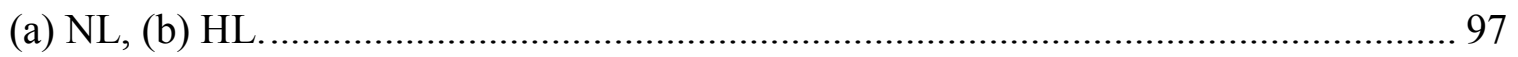

Figure 4.22: Overlaid selected pressure traces from casing Kulites, NL blue, HL red. Flow

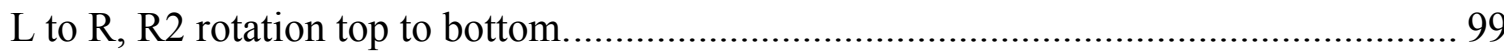

Figure 4.23: FT spectra of casing Kulites, NL, with focus on R2 BPF spectra. Flow L to R,

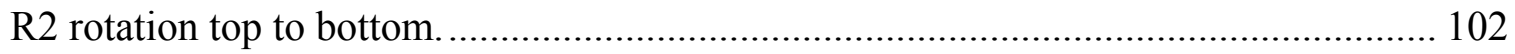

Figure 4.24: FT spectra of casing Kulites, HL, with focus on R2 BPF spectra. Flow L to R,

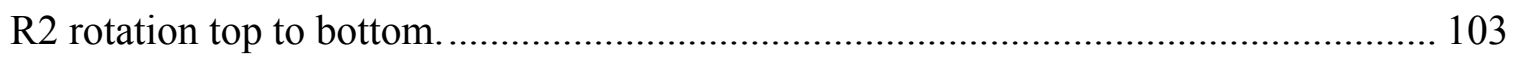

Figure 4.25: FT spectra of casing Kulites, NL, with focus on R1 and R3 BPF spectra. Flow

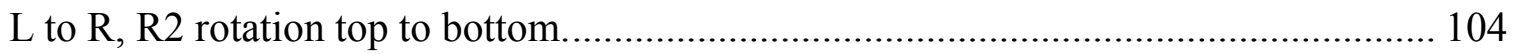

Figure 4.26: FT spectra of casing Kulites, HL, with focus on R1 and R3 BPF spectra. Flow

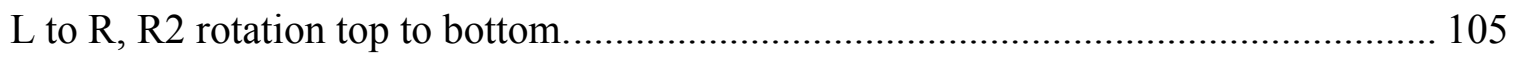

Figure 4.27: Agilis NSMS software output showing relative amplitude of blades

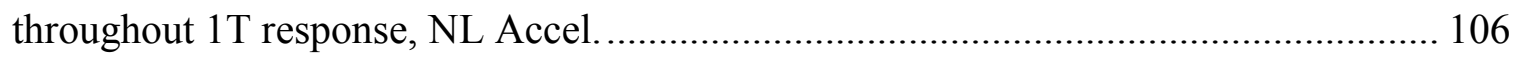

Figure 4.28: Rotor 2 maximum peak-to-peak deflection per blade. ........................... 107

Figure 4.29: P-P response of each R2 blade at NL, overlaid.................................. 108

Figure 4.30: P-P response of each R2 blade at HL, overlaid................................... 109

Figure 4.31: Rotor 2 frequency at point of maximum p-to-p deflection per blade........ 110

Figure 4.32: Rotor 2 damping coefficient, $\mathrm{C} / \mathrm{Cc}$, per blade..................................... 111

Figure 4.33: ND response from LSMF analysis on Agilis software for NL................. 114 
Figure $\quad$ Page

Figure 4.34: ND response from LSMF analysis on Agilis software for HL................. 115

Figure 4.35: S1 Exit EA of Alpha and FFT of EA, 10\% VP. (a) 50\% Span, NL, (b) 50\%

Span, HL, (c) 80\% Span, NL, (d) 80\% Span, HL.................................................. 117

Figure 4.36: S1 Exit EA of Alpha and FFT of EA, 55\% VP. (a) 50\% Span, NL, (b) 50\%

Span, HL, (c) 80\% Span, NL, (d) 80\% Span, HL.................................................. 118

Figure 4.37: R2 Exit EA of Alpha and FFT of EA, 45\% VP. (a) 50\% Span, NL, (b) 50\%

Span, HL, (c) 80\% Span, NL, (d) 80\% Span, HL.................................................. 119

Figure 4.38: R2 Exit EA of Alpha and FFT of EA, 85\% VP. (a) 50\% Span, NL, (b) 50\%

Span, HL, (c) 80\% Span, NL, (d) 80\% Span, HL................................................ 120

Figure 4.39: Mean pressures on SS and PS of Stator 2, NL................................... 121

Figure 4.40: Mean pressures on SS and PS of Stator 2, HL.................................... 122

Figure 4.41: Stator Kulite blade loading as a function of rotor position, 50\% Span NL.123

Figure 4.42: Stator Kulite blade loading as a function of rotor position, 50\% Span HL.124

Figure 4.43: Stator Kulite blade loading as a function of rotor position, 80\% Span NL.125

Figure 4.44: Stator Kulite blade loading as a function of rotor position, 80\% Span HL.126

Figure 4.45: FT Spectra of pressure side Stator Kulites, NL................................... 127

Figure 4.46: FT Spectra of pressure side Stator Kulites, HL .................................... 128

Figure 4.47: FT Spectra of suction side Stator Kulites, NL.................................... 128

Figure 4.48: FT Spectra of suction side Stator Kulites, HL...................................... 129

Figure 5.1: Calculated time between square wave pulses from the $1 / \mathrm{rev}$ channel as a function of revolution number for a data run accelerating through R2 1T................. 137

Figure 5.2 Sample blade pass signatures from casing Kulites for each revolution. ....... 138 
Figure

Figure 5.3: Example ensemble average of one blade pass from a revolution of Kulite data, HL, 3680 RPM, casing Kulite.

Figure 5.4: Comparison of blade-to-blade method of maximum blade pass pressure amplitude, NL decel Casing Kulites.

Figure 5.5: Blade 13 passage seen by casing Kulites acquired on different PXIE cards.

Figure 5.6: 400 successive revolutions shown of a blade pass, illustrating lag offset.... 143 Figure 5.7: Zoomed in image of Figure 5.6. Lag offset per revolution is only a fraction of a sample period.

Figure 5.8: Kulite blade passage shape showing multiple revolutions for sensor on same card as $1 /$ rev trigger.

Figure 5.9: Lag offset is also apparent in close up of $42 \mathrm{~Hz}$ sine wave. Channel 17 is plotted, and the " $1 /$ rev" sine wave used to parse the signal was taken to be Channel 0 . 146 Figure 5.10: Sine wave from channel 0, showing no offset, since it was acquired on the same card as the designated " $1 / \mathrm{rev"} \mathrm{signal.}$ 146

Figure 5.11: Sample number at 6V trigger plotted as a function of Rev \#, with a LSF line applied to the data. 147

Figure 5.12: Several consecutive channels plotted to show parallel LSF slopes...... 148 Figure 5.13: Time offset normalized by seconds, plotted as a function of channel number. The drift is nearly constant along all channels and acquisition time. 148 Figure 5.14 Illustration of consecutive addition of time lag offset for each successive sample. 150 
Figure $\quad$ Page

Figure 5.15: Blade 13 passage as seen by casing Kulite, after lag offset corrections. ... 151

Figure 5.16: Example of successive revolutions of data used for successive FFT generation. 154

Figure 5.17: FFT generated for each revolution of data in Figure 5.16....................... 155

Figure 5.18: Same FFT as in Figure 5.17, but zero padded to length of 30,000 points. 155 Figure 5.19: FFT Stator Waterfall, Kaiser Window, PS, 80\% Span, 10\% Chord, NL... 158 Figure 5.20: FFT Stator Waterfall, Kaiser Window, PS, 80\% Span, 20\% Chord, NL... 159 Figure 5.21: FFT Stator Waterfall, Kaiser Window, PS 80\% Span, 30\% Chord, NL.... 159 Figure 5.22: FFT Stator Waterfall, Kaiser Window, PS 80\% Span, 40\% Chord, NL... 160 Figure 5.23: 99/rev FFT amplitude, PS 80\% Span, 10\%-40\% Chord, NL................... 160 Figure 5.24: 66/rev FFT Amplitude, PS 80\% Span, 10\%-40\% Chord, NL................... 161 Figure 5.25: FFT Stator Waterfall, Kaiser Window, PS 80\% Span, 10\% Chord, HL... 162 Figure 5.26: FFT Stator Waterfall, Kaiser Window, PS 80\% Span 20\% Chord, HL..... 163 Figure 5.27: FFT Stator Waterfall, Kaiser Window, PS 80\% Span 30\% Chord, HL.... 163 Figure 5.28: FFT Stator Waterfall, Kaiser Window, PS 80\% Span, 40\% Chord, HL.... 164 Figure 5.29: 99/rev FFT amplitude, PS 80\% Span, 10\%-40\% Chord, HL.................... 165 Figure 5.30: FFT Stator Waterfall, Kaiser Window, PS 50\% Span, 10\% Chord, NL.... 166 Figure 5.31: FFT Stator Waterfall, Kaiser Window, PS 50\% Span 40\% Chord, NL.... 166 Figure 5.32: 66/rev FFT amplitude, PS 50\% Span, 10\% and 40\% Chord, NL ............. 167 Figure 5.33: 99/rev FFT amplitude, PS 50\% Span, 10\% \& 40\% Chord, NL............... 167 Figure 5.34: FFT Stator Waterfall, Kaiser Window, SS 80\% Span 10\% Chord, NL.... 168 Figure 5.35: FFT Stator Waterfall, Kaiser Window, SS 80\% Span 40\% Chord, NL.... 169 
Figure

Figure 5.36: 99/rev FFT Amplitude, SS 80\% Span 10\% \& 40\% Chord, NL 169

Figure 5.37: Stator Waterfall, Kaiser Window, SS 50\% Span, 10\% Chord, NL........... 170

Figure 5.38: FFT Stator Waterfall, Kaiser Window, SS 50\% Span 40\% Chord, NL.... 170

Figure 5.39: 99/rev FFT Amplitude, SS 50Span, 10\% \& 40\% Chord, NL. .................. 171

Figure 5.40: Relative position of instrumented stator section to window Kulite measurements. 176

Figure 5.41: Standard Deviation, Blade 26, PS 80\% Span 20\% Chord, HL. ................. 179

Figure 5.42: Cross Correlation, Blade 26, PS 80\% Span 20\% Chord, HL.................... 179

Figure 5.43: Cross Covariance, Blade 26, PS 80\% Span 20\% Chord, HL.................... 180

Figure 5.44: NSMS Peak to Peak Amplitude, Blade 26, HL...................................... 180

Figure 5.45: Rotor blade passage traces, Blade 26, PS 80\% Span 20\% Chord, HL...... 181

Figure 5.46: Cross Covariance, Blade 26, PS 50\% Span 10\% Chord, HL................... 182

Figure 5.47: Cross Covariance, Blade 26, PS 80\% Span 40\% Chord, HL................... 182

Figure 5.48: Cross Covariance, Blade 26, PS 80\% Span 20\% Chord, NL................... 183

Figure 5.49: NSMS Peak to Peak Amplitude, Blade 26, NL.................................... 183

Figure 5.50: Rotor blade passage traces, Blade 26, PS 80\% Span 20\% Chord, NL...... 184

Figure 5.51: Rotor blade passage traces, Blade 26, SS 80\% Span 10\% Chord, HL....... 185

Figure 5.52: Cross Covariance, Blade 26, SS 80\% Span 10\% Chord, HL................... 185

Figure 5.53: Cross Covariance, Blade 13, PS 80\% Span 20\% Chord, HL.................... 186

Figure 5.54: NSMS Peak to Peak Amplitude, Blade 13, HL................................... 187

Figure 5.55: Rotor blade passage traces, Blade 13, PS 80\% Span 20\% Chord, HL...... 187

Figure 5.56: NSMS Peak to Peak Amplitude, Blade 8, HL....................................... 188 
Figure $\quad$ Page

Figure 5.57: Cross Covariance, Blade 8, PS 80\% Span 20\% Chord, HL ...................... 188

Figure 5.58: Cross Covariance, Blade 22, PS 80\% Span 20\% Chord, HL.................... 189

Figure 5.59: NSMS Peak to Peak Amplitude, Blade 22, HL...................................... 190

Figure 5.60: NSMS Peak to Peak Amplitude, Blade 27, HL...................................... 191

Figure 5.61: Cross Covariance, Blade 27, PS 80\% Span 20\% Chord, HL.................... 191

Figure 6.1: Rotor 1 forward looking aft total displacement from nominal, mm. .......... 208

Figure 6.2: Rotor 1 Aft looking forward total displacement from nominal, mm. ......... 209

Figure 6.3: R2 forward looking aft total displacement from nominal, mm................. 209

Figure 6.4: R2 Aft looking forward total displacement from nominal, mm................ 210

Figure 6.5: R3 Forward looking aft total displacement from nominal, mm................. 210

Figure 6.6: R3 Aft looking forward total displacement from nominal, mm................ 211

Figure 6.7: Dantec 3D Hotwire Probe Type 55-14677501. Illustration courtesy Dantec

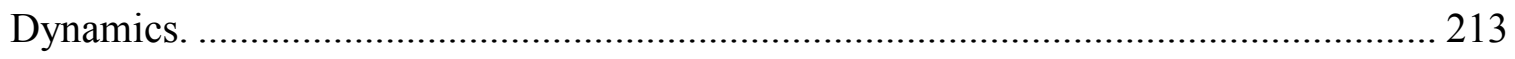

Figure 6.8: View of 3D calibration space in which pitch wise voltage variation is illustrated

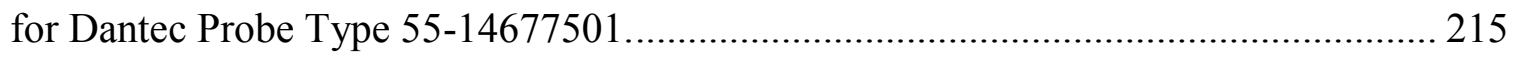

Figure 6.9: EA S1 exit data away from vane wake, NL, 50\% Span, showing unnaturally high pitch angles as a result of calibration drift in hardware. No temp or density

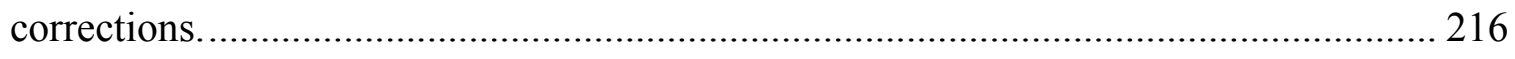

Figure 6.10: 3D Calibration space of Dantec Type 55-Crossflow probe. ..................... 217

Figure 6.11: 3D Calibration space of Dantec Type 55-14677501 probe...................... 218

Figure 6.12: Comparison of Kaiser, Hanning, and Hamming windows....................... 219

Figure 6.13: Stator Kulite 80\% Span 40\% Chord, no windowing, large range............. 220 
Figure 6.14: Same data as Figure 6.13, reduced range, no windowing. 220

Figure 6.15: Same data as Figure 6.14, Kaiser windowing. ..................................... 221

Figure 6.16: Same data as Figure 6.14, Hamming windowing................................... 221

Figure 6.17: Same data as Figure 6.14, Hanning windowing. ................................... 222

Figure 6.18: Same data as Figure 6.15, subtract out steady spectra, Kaiser windowing. 222

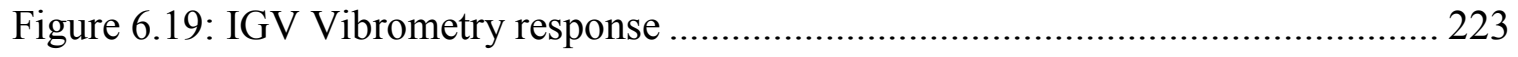

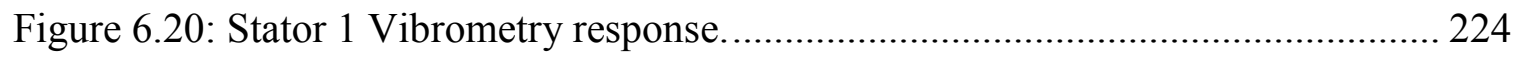

Figure 6.21: Stator 2 Medium Section Vibrometry response. ................................... 224

Figure 6.22: Stator 2 Large Section Response.................................................... 225

Figure 6.23: Stator 2 Small Section Response..................................................... 225

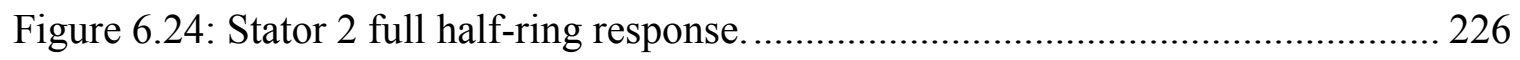

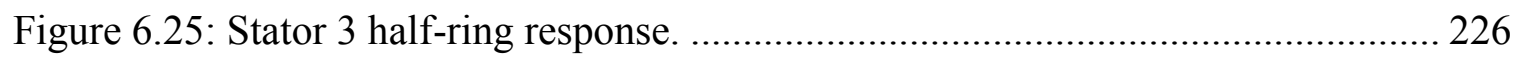




\begin{tabular}{|c|c|}
\hline$\underline{\text { Symbol }}$ & $\underline{\text { Definition }}$ \\
\hline $1 \mathrm{~T}$ & $1^{\text {st }}$ Torsion \\
\hline $316 \mathrm{SS}$ & 316 Stainless Steel \\
\hline $\mathrm{ADC}$ & Analog-Digital Converter \\
\hline AIP & Aerodynamic Interface Plane \\
\hline APU & Auxiliary Power Unit \\
\hline $\mathrm{BPF}$ & Blade Pass Frequency \\
\hline BPP & Blade Pass Period \\
\hline CFD & Computational Fluid Dynamics \\
\hline $\mathrm{CL}$ & Clocking \\
\hline CWB & Chord-Wise Bending \\
\hline DAQ & Data Acquisition \\
\hline EA & Ensemble Average \\
\hline EDM & Electrical Discharge Machining \\
\hline EO & Engine Order \\
\hline FAT & Factory Acceptance Test \\
\hline FEA & Finite Element Analysis \\
\hline FS & Full Scale \\
\hline
\end{tabular}




\begin{tabular}{|c|c|}
\hline FT & Fourier Transform/Fast Fourier Transform \\
\hline GPM & Gallons per Minute \\
\hline $\mathrm{HCF}$ & High Cycle Fatigue \\
\hline $\mathrm{HL}$ & High Loading \\
\hline HP & Horsepower \\
\hline IBR & Integrally Bladed Rotor \\
\hline IGV & Inlet Guide Vane \\
\hline LSMF & Least Squares Model Fitting \\
\hline LDV & Laser Doppler Velocimetry \\
\hline M & Mach Number \\
\hline $\mathrm{N}_{\mathrm{m}}$ & Mechanical Rotational Speed \\
\hline $\mathrm{N}_{\mathrm{c}}$ & Corrected Rotational Speed \\
\hline ND & Nodal Diameter \\
\hline NI & National Instruments \\
\hline NL & Nominal Loading \\
\hline NPT & National Pipe Thread \\
\hline NSMS & Non-Intrusive Stress Measurement System \\
\hline ODE & Ordinary Differential Equation \\
\hline $\mathrm{PH}$ & Precipitation Hardened \\
\hline PID & Proportional-Integral-Derivative \\
\hline P-P & Peak to Peak \\
\hline PS & Pressure Side \\
\hline $\mathrm{R}$ & Gas Constant \\
\hline
\end{tabular}




\begin{tabular}{|c|c|}
\hline $\mathrm{R} 1 / \mathrm{R} 2 / \mathrm{R} 3$ & Rotor $1 /$ Rotor $2 /$ Rotor 3 \\
\hline RPM & Rotations per Minute \\
\hline RTV & Room Temperature Vulcanizing silicone \\
\hline $\mathrm{S} 1 / \mathrm{S} 2 / \mathrm{S} 3$ & Stator $1 /$ Stator $2 /$ Stator 3 \\
\hline SAR & Super Abrasion Resistant \\
\hline SCFM & Standard Cubic Feet per Minute \\
\hline SS & Suction Side \\
\hline $\mathrm{SSCC}$ & Single Stage Centrifugal Compressor \\
\hline $\mathrm{TC}$ & Thermocouple \\
\hline TPR & Total Pressure Ratio \\
\hline VFD & Variable Frequency Drive \\
\hline VP & Vane Passage \\
\hline$a_{o}$ & Speed of Sound in Air \\
\hline$\alpha$ & Absolute Flow Angle w.r.t Compressor Axis \\
\hline$A$ & Amplitude of Torsion Vibration \\
\hline$\beta$ & Relative Flow Angle w.r.t Compressor Axis \\
\hline$B$ & Number of Blades \\
\hline $\mathrm{c}$ & Chord, Damping \\
\hline$c_{x y}$ & Covariance Term \\
\hline $\mathrm{C} / \mathrm{Cc}$ & Damping Coefficient \\
\hline$C_{v}$ & Flow Coefficient \\
\hline $\mathrm{E}$ & Voltage \\
\hline$F_{s y}, F_{s u}$ & Yield Strength, Ultimate Strength \\
\hline
\end{tabular}




\begin{tabular}{ll}
$G_{g}$ & Gas Specific Gravity \\
$\mathrm{k}$ & Wave Number, Spring Constant \\
$l b_{f}, l b_{m}$ & Pound Mass/Pound Force (US) \\
$\mathrm{m}$ & Integer, Harmonic Number, Mass, Lag Offset \\
$\dot{m}$ & Mass Flow Rate \\
$\mathrm{N}$ & Vector Length \\
$N_{2}$ & Constant for Unit Conversion \\
$N_{c}, N_{m}$ & Corrected Speed, Mechanical Speed \\
$\mathrm{P}, \mathrm{p}$ & Static Pressure \\
$\mathrm{P}_{\mathrm{o}}$ & Stagnation Pressure \\
$\mathrm{P}_{\mathrm{o}, \mathrm{AA}}$ & Stagnation Pressure, Area Averaged \\
$\phi$ & Maximum Torsion Angle in Radians \\
$\mathrm{q}$ & Volumetric Flow Rate \\
$\rho_{0}$ & Stagnation Air Density \\
$\hat{R}_{x y}$ & Cross Covariance Term \\
$\sigma$ & Interblade Phase Angle \\
$T_{w}$ & Stagnation Temperature \\
$\mathrm{T}$ & Rotor \\
\hline $\mathrm{V}$ & Absolute Frame Velocity, Voltage \\
\hline &
\end{tabular}


VP

$\omega$

$\omega$ W
Vane Passage

Torsional Vibration Frequency

Doppler Shifted Torsional Vibration Frequency

Velocity in the Rotor Relative Reference Frame 


\begin{abstract}
Murray III, William L. M.S.A.A., Purdue University, May 2014. Experimental Investigation of a Compressor Forced Response Condition in a Multistage Compressor. Major Professor: Nicole L. Key.
\end{abstract}

The objective of this research is twofold. Firstly, the design, development, and construction of a test facility for a Honeywell APU-style centrifugal compressor was implemented, as well as the design and construction of an inlet flow experiment. Secondly, the aeromechanical response of an embedded stage in the Purdue 3-Stage axial research compressor was analyzed through a suite of different measurement techniques in the fulfillment of the end of the GUIde IV Consortium contract.

The purpose of the first phase of Honeywell work was to comprehensively measure the flow field of an APU-style centrifugal compressor inlet through the use of Laser Doppler Velocimetry (LDV). A portion of a Honeywell supplied inlet was modified to provide optical access to the elbow, and a gas ejector system was designed and constructed to provide the same suction to the inlet that it would see during operation with the compressor. A performance and health monitoring electronics system was designed and purchased to support the testing of the Honeywell inlet ejector system and eventually it will be used for testing with a centrifugal compressor. 
Additionally, a secondary air and oil system has been designed and is currently being constructed in the test cell in preparation for the arrival of the Honeywell compressor this summer.

An embedded rotor stage in the Purdue 3-stage compressor, with a Campbell diagram crossing of the $1 \mathrm{~T}$ vibratory mode was analyzed with a suite of measurement systems. In addition to steady state compressor performance measurements, other types of measurements were used to characterize the aerodynamic forcing function for this forced response condition including: NSMS, high-frequency pressure transducers mounted in the casing and in a downstream stator, and cross-film thermal anemometry. Rotor geometry was measured by Aerodyne using an in-situ laser scanning technique. Vibrometry testing was performed at WPAFB to characterize safe operating speeds for stator vibrations.

Several unsteady data processing techniques were developed to analyze the fastresponse pressure and hot film data. Since it was unsafe to operate the compressor at R2 resonance, slow sweeps through the resonance were utilized, thus complicating the data processing strategy. After significant analysis, there is evidence of the R2 vibration in the fast-response pressure measurements using frequency and time-based analysis methods. Although not used in final data acquisition, the 3D hotwire calibration facility and data processing techniques have been improved. The overall purpose of the work is to create a detailed dataset centered on the forced response of $\mathrm{R} 2$ at the 1T Campbell diagram crossing to help further the development and validation of predictive aeromechanic simulations of axial compressors. 


\section{CHAPTER 1. INTRODUCTION}

\subsection{Motivation}

Srinivasan (1997), in a recent paper on vibrations in turbomachinery, quotes Hayne Constant, one of the first jet aircraft engineers in WWII, as saying, "On axial compressor blade vibration we have gone very cautiously and I believe have not met the problem in all its bleakness; the dark days are still ahead". Today, almost 70 years later, engineers and researchers are still continuing to forge ahead in developing methods to fully understand and mitigate the effects of axial compressor blade vibration.

Axial compressor turbomachinery has experienced numerous changes in design and manufacturing since its invention, with vibration being a key driving factor in many of the choices made in the development of new technology. Compressor design has trended toward higher pressure ratios, integrated bladed rotors (IBR), higher temperatures, and complex instrumentation including advanced health monitoring electronics that create an extremely complex and harsh environment for hardware from a vibration perspective. Higher pressure ratios create stronger forcing environments, higher temperatures combined with higher vibrations drive material requirements and IBRs have extremely low mechanical damping. However, high-speed electronics allow for close monitoring of vibration modes and High Cycle Fatigue (HCF). 
IBR assemblies have recently risen significantly in popularity, as has research surrounding them. IBRs, also known as blisks, consist of a solid machined blade and disk assembly, meaning that the blades are joined together as one structure. They have many benefits, such as reduced manufacturing time, reduced assembly complexity, and reduced weight compared to a traditional bladed rotor where each blade must be securely fastened to the rotating disk. However, due to the fact that a IBR is machined from one solid metal material, both blade and Nodal Diameter (ND) mode vibrations are amplified. The differences in the vibration characteristics between traditional rotor designs and blisks is significant - with the former, there is typically some mechanical damping due to the friction of the "fir tree" of each blade within its respective disk slot. However, IBRs must rely solely on aerodynamcs for any significant damping, as there there is little to no mechanical contribution.

It is important to accurately predict the amplitudes of Campbell diagram crossings, as it is impossible to avoid all crossings in a modern multistage compressor. HCF as a result of excessive vibration or dwelling at an engine speed with a significant vibratory response for an extended period of time can ground an entire fleet of aircraft or result in costly delays in engine development. Thus, it is extremely important to develop tools that can be accurately used to predict vibration amplitudes during the engine design process, and it is necessary to gather datasets that can be used to validate these tools for use in multistage compressors. 


\subsection{Forced Response Aeromechanics}

Forced excitation occurs from the uneven loading on the IBR or individual blade that occurs in the same circumferential position for each revolution of the hardware. It could be caused by upstream stator wakes, struts, or inlet distortion, as well as potential fields arising from downstream rotors or stators.

In the realm of turbomachinery forced response, a Campbell diagram is a useful tool for engineers to predict when engines could encounter potentially dangerous vibrations. Plotted on the abscissa is shaft speed, and on the ordinate, frequency. Known engine mode frequencies are plotted as a function of RPM. Centrifugal stiffening can cause the modal frequency to increase slighty with compressor shaft speed. Engine order lines are plotted, starting from the origin and extending outward. Engine order lines represent all possible excitations that could be produced as a function of rotational speed. Where an EO line and a mode line intersect, there is potential for a resonant response - the excitation frequency of the compressor is matching the natural frequency of the engine component of interest. With the use of campbell diagrams it is relatively straightforward to predict potential excitations if specific mode frequencies are known and the EOs of concern are known to excite the frequency of interest. However, it is difficult to predict the amplitude of the response and the damping associated with the vibration. Figure 1.1 shows an example Campbell Diagram for a centrifugal compressor impeller, where 4 Engine Orders are shown, and three impeller modes, denoted by F1, F2, and F3. The largest experimentally measured response, the crossing of EO 30 and F1. 


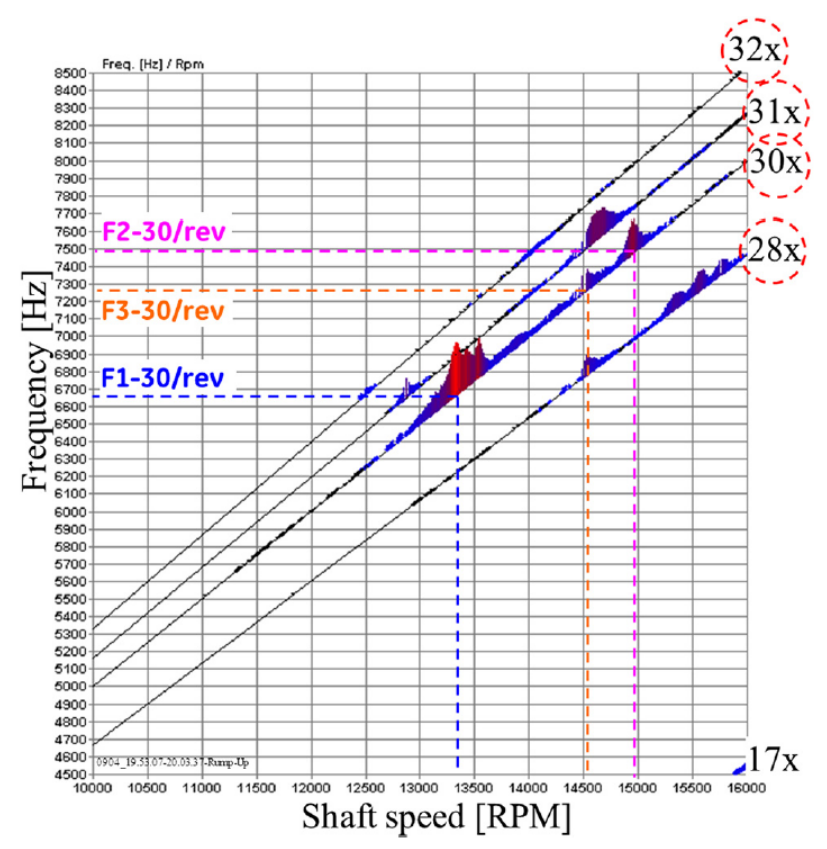

Figure 1.1: Campbell diagram showing 4 EOs and three modes, Richards et al. (2012).

One of the largest difficulties in predicting the amplitudes of resonant EO crossings is due to the complex nature of predicting aerodynamic forcing and damping effects. For example, a common modeling approach for an IBR is to treat all blades as $2^{\text {nd }}$ order ODE harmonic oscillators, accoring to Eqn. 1-1, where $m, c$, and $k$ are the mass, damping, and stiffness of the object, respectively. The unsteady cyclic forcing function that will drive the response of the object is given as $F e^{i \omega t}$.

$$
m \ddot{x}+c \dot{x}+k x=F e^{i \omega t}
$$

The unsteady cyclic forcing can come from stator vane wakes, gusts, or potential fields, as well as from non-aerodynamic sources, such as bearings or gear meshes. Regardless of the source, predicting the real-world amplitude of the forcing fucntion is the 
most difficult part of predicting forced response and one of the main focuses in aeromechanics today.

Multistage compressors exhibit interesting characteristics that are not seen within a single stage rig. Most derived equations pertaining to aeromechanic forcing or damping response of a single isolated vibrating blade row are devoid of any acoustic interaction with nearby stationary airfoils or struts. Mengle (1990) was able to derive relationships that show how blade vibration frequency shifts when detected in a stationary frame of reference, and he was able to show how the frequencies would be shifted as they are reflected from nearby stator or rotor rows, expanding to a stage and a half configuration. He remarked that more experimental work should be conducted to verify his multistage predictions. Hall and Silkowski (1997) created analytical methods that showed calculations of changes in unsteady lift due to forced response and how the perturbations to normal flow would have an effect on the vibratory patterns of a rotor in forced response.

There is another phenomenon that has been observed in fan blades, something that Hanson (1993) has termed "Mode Trapping." Mode trapping arises when there is a strong interaction between frequency spreading and reflection due to a rotor-stator interaction that can cause the relative spectral amplitudes of higher-order harmonics of blade pass frequency to grow or shrink based on the speed of the passing blades. He was able to create a matrix of equations that can be solved for the acoustic response of a trapped mode in a fan due to the scattering of blade-pass frequency information propagating upstream and downstream from a rotating fan blade. Both the shape and acoustic reflectivity of the stators and nozzle can affect the strength of the trapping effect and can cause a strengthening or dampening of blade pass harmonics. 
Blisk or blade mistuning is something that researchers and manufacturers have started using to reduce the aerodynamic response of blades in resonance. There are different methods that can accomplish this task, and they fall into two categories: by mass addition or by mass subtraction. Kaza and Kielb (1982) developed an analytical model where multidegree-of-freedom relationships were used to create a model of a fully mistuned bladed disk to better understand the effects of mistuning on wake-induced forced response and coupled bending-torsion flutter modes. Through mistuning, they could reduce the flutter response of a blisk, or speed up the circumferential propagation of flutter, but it could have either positive or negative effects on the amplitude of forced response.

Buffum (1995) created a computer model that predicted the effects of blade row interactions on flutter and forced response with a linearized model of a rotor-stator pair. In the specific case of analyzing forced response, he was able to show how the locality of the impinging rotor wakes from an upstream rotor would affect the resonant response of a stator section, showing that the stator vibrational amplitude response could vary from $15 \%$ to $60 \%$ that of non-aerodynamically coupled response by changing the gap between rotor and stator from 0 chord lengths to 1 chord length. The positioning of wakes, gusts, or shocks can change response patterns, and Im and Zha (2013) discussed how a CFD simulation of a transonic fan showed that the chordwise position of where a shock occurs is able to change when flutter occurs, by creating flow blockage within a fan blade passage. $\mathrm{Li}$ and $\mathrm{He}$ (2005) also conducted a similar analysis that agreed well with the work conducted by Buffum (1995) showing that the aerodynamic dampening on a rotating blade row is a significant function of the rotor-stator gap. If the rotor-stator gap is increased, the damping will decrease to a minimum before beginning to increase again, but it will never 
return to the minimum damping level. This effect is partially due to the interaction of rotor resonant reflections on stator vanes, and this interaction is known to also effect the blade vibration amplitude. Because of this, the amount of blade vibration damping due to stator reflections will also be a function of stator vane count, indicating that an optimal combination of vane number and rotor-stator gap exists that will minimize forced response.

Salontay et al. (2011) conducted an experimental and computational study on the Purdue 3-stage axial compressor and showed that the response of an embedded rotor stage undergoing forced response was a function of the stator clocking geometry around the responding rotor. In particular, they found that computational models were able to accurately predict the ratio of response amplitudes between different stator vane clocking positions, and maximum blade vibration amplitudes could be decreased by up to $37 \%$ by changing vane clocking configurations.

The effects of forced response modes are not limited to simply axial machines. Richards et al. (2012) conducted a CFD and experimental investigation on a GE oil and gas centrifugal compressor stage fed by a radial inlet with deswirl vanes followed by a centrifugal impeller and vaneless diffuser. Through the use of strain gauges, they were able to discover a $30 /$ rev excitation that was driven by interactions between the impeller and wakes reflected off of downstream return channel vanes. These reflected wakes would then impinge on the trailing edge of the impeller, and a spectral analysis of the CFD and strain gauge data showed this excitation was the greatest at the impeller trailing edge. There was also a significant 14/rev excitation at the leading edge of the impeller due to the interaction with upstream inlet deswirl vane wakes. 


\subsubsection{Mistuning Effects}

Mistuning, intentional or not, has an enormous effect on the response of a blisk, and it has been studied quite extensively in recent years. Researchers have devised many methods of determining the optimal mistuning patterns for different designs of blisks, usually resorting to a Monte Carlo-type analysis, where the characteristics of every blade in a bladed disk design are altered randomly to give either the best or worst possible mistuning pattern within a statistical bound of physically possible configurations. The loss of the assumption of cyclic symmetry when analyzing a mistuned disk creates a more computationally intensive problem, and if CFD is utilized, it usually requires a very large grid to achieve results.

Firstly, the direct result of mistuning is the spreading of the blades' response to several different frequencies, where separation in the frequency domain is proportional to the amount of mistuning between each blade. In one of the pioneering efforts in investigating mistuning, Ewins (1968) showed how blade mistuning will cause the frequency range of the response of a blisk to increase in width, as well as peak at different frequencies. The main response of the blisk assembly will have a maximum at a given frequency, followed by a smaller, separate peak at a higher frequency. His work also showed that mistuning can increase blade stress by up to $20 \%$ above a non-mistuned blisk system, and this increase in blade stress should be assumed to occur in all production engines based on manufacturing defects and wear over the lifetime of the engine. Although computational methods exist that are capable of estimating the amount of mistuning in a blisk assembly and the resulting increase in stress, it is still difficult to predict real-world 
mistuning, as well as real world stress amplitude increases due to mistuning, given the complex flow environment in an operating engine.

Kaneko (2013) showed that the speed at which a mistuned bladed disk was accelerated through resonance was related to the amplitude of the transient vibration that it experienced. In particular, he studied a mistuned blisk where the $1^{\text {st }}$ mode natural ND frequencies were far removed from the neighboring modes and relatively constant. A massspring-damper model and an FEA model of the blisk were simulated, and the decrease in blade vibration responses was proportional to an increase in acceleration rate, and this proportionality between the blades was not the same from blade to blade. The higher responding blades decreased in amplitude faster with respect to an increase in acceleration rate. Additionally, the highest reduction in response occurred for ND type vibrations, or blisk response, because the reduction in vibration response appears to be inversely proportional to the amount of damping.

Zhai et al. (2012) showed that the mistuning of a bladed disk will have different effects on both forced response and flutter. CFD studies illustrated that as little as $1 \%$ frequency response offset per blade is enough to significantly decrease flutter amplitude and have a beneficial effect on forced response. It was remarked, however, that intentional mistuning can have a beneficial or detrimental effect on rotor forced response, although the mistuning pattern discussed was one with slight benefits.

\subsubsection{Forced Response Measurement Techniques}

Measuring vibration response in turbomachinery can be accomplished by a number of measurement techniques including, but not limited to, strain gauges, pressure 
transducers, microphones, non-intrusive measurement techniques (NSMS), and accelerometers.

Strain gauge techniques can be employed in a number of ways, but the most common technique involves mounting strain gauges on the blades of the rotating turbomachinery and using a slip-ring device to transmit the signals from the strain gauges to the recording equipment. This measurement is much easier if instead the strain gauges are mounted to stationary hardware, such as a stator vane or used in a cascade experiment, similar to Freund et al. (2013).

Pressure transducers can be mounted either on a stationary part of the machine, most often the casing wall, similar to work conducted by Baumgartner et al. (1995), or they can be mounted on rotating machinery, for example or Gill and Capece (2004), where the signal would be transmitted through a slip ring device to a recorder. The latter is a more difficult measurement, and therefore, most pressure measurements have been made using a stationary source. To resolve vibration frequencies occurring in a rotating reference frame, one must Doppler shift frequency data from the rotating to the stationary reference frame similar to work conducted by Mengle (1990) and Kurkov (1981).

Reducing noise and unwanted frequency information from pressure transducers is a non-trivial operation, and it must be completed to ensure a good signal to noise ratio, especially in the case of forced response where pressure response amplitudes are low. One method of reducing unwanted noise is to take a pressure trace of the engine while it is operating in a condition without vibrations, to record engine-order and blade pass related frequency information, which can be used as a reference signal. Kurkov (1981) showed that the spectrum of this non-vibrating signal can be subtracted from the spectrum of the 
pressure signal measured during the forced response vibration to view the frequency spectrum of forced response. This method is effective at reducing blade pass frequency related noise, but it doesn't fully get rid of it. This method has also been employed by Mengle (1990) in his attempt at removing integral engine order frequencies from observed spectra of a rotating compressor blade.

Kurkov (1981) was able to take an average frequency response of 16 revolutions of data without vibration and subtract that frequency spectrum from the vibratory response spectrum. He was also able to take a representative blade pass pressure signature from a non-vibrating blade and subtract this pressure information from the blade pass signature during vibration on a blade-by-blade basis. This resulting pressure trace was then used in a spectral analysis to understand the effect of blade vibration.

Rotating stall and flutter have been studied extensively in the realm of aeromechanics. When compressor blades go into stall or experience flutter, the mechanism of vibration is not tied to a phenomenon linked to compressor shaft speed, and thus, the vibration frequency will not be a multiple of the rotation frequency of the compressor. Stall and flutter have generally much larger vibration amplitudes, and correspondingly larger pressure wave amplitudes, making them easier to measure with pressure transducers. Liechtfu $\beta$ et al. (2013) measured non-engine order flutter spectral signatures due to stall in stationary Kulite pressure transducers from a blisk-construction compressor rotor blade. Schoenenborn and Breuer (2012) measured torsion and flutter of blades during surge, and was easily able to detect aeroacoustic signatures from unsteady pressure transducers related to blade motion in this flow regime, as well as relate it to NSMS tip timing blade deflection 
data. However, the aerodynamic flow regime inside a compressor during surge is much different from the flow regime during a forced response, as is the focus of this thesis.

It is not known to the author if any reasearchers have directly measured low amplitude engine-order forced response directly through the use of stationary pressure transducers in an experimental compressor facility, as the amplitudes of the acoustic waves are generally very small in comparison to other flow field features. Most research conducted to this point has been focused on flow measurement tied to non-integral engine order vibrations, such as stall, flutter, surge, or acoustic resonances. Fridh et al. (2013) measured the spectral component of forced response from partial admission inlet distortion in a turbine using pressure transducers in a rotating reference frame. They were able to detect resonance at campbell crossings and create campbell diagrams from strain gauge and pressure transducer data. However, the strain gauge data showed much more detailed spectral data on the response characteristics of important campbell crossings compared to the pressure data.

Baumgartner et al. (1995) used a single hot wire film downstream of the tip of a vibrating rotor blade and some casing-mounted Kulite XTE-190 pressure transducers to investigate rotating stall and flutter modes of rotating blades. They found that the spectral magnitudes of the instability or vibration was significantly smaller in amplitude than the spectral magnitude related to blade passing frequency.

Macro-fiber composites have been used both as a blade excitation and a blade strain measurement system. They are made of rectangular rods oriented parallel to each other in a planar direction, and are sensitive to strain in that particular direction, and made into thin sheets. If a voltage is applied to them, they can expand and contract. Belz et al. (2013) used 
them to excite fan blades in an aeroelastically unstable fashion to simulate blade vibration shapes that have been known to cause failure, as opposed to using an aerodynamic excitation. This is useful if one would like to only study the aerodynamic flowfields generated by blade motion without a forcing aerodynamic flowfield that could make measurements difficult. They also instrumented the same blades with Kulite pressure transducers and strain gauges to measure blade deformation and unsteady blade pressure fields.

\subsection{Effects of Steady Inlet Distortion on Centrifugal Compressors}

Centrifugal compressors are used in a breadth of applications ranging from refrigeration pumps to high-powered aircraft engines and as such, are subject to a wide range of airflow conditions at the inlet to the compressor. Inlet distortion can be defined as any property of the inlet flow that causes part of the flow to become non-uniform, most commonly categorized into pressure, temperature, or flow angle distortion. This distortion can come from any number of sources, usually categorized into transient or steady flow distortion, depending on whether or not the distortion is transient (exists for less than approximately one rotor revolution), or steady, (lasting for a significant number of blade rotations). Most inlet distortion research is concerned with the effects of steady inlet distortion, as transient distortions usually have less of an effect on compressor performance and are particularly difficult to measure. Only steady inlet distortion research will be presented.

The non-uniformity of inlet air flow is usually detrimental to many aspects of the performance of a centrifugal compressor. Flow distortion leads to a decrease in stall and 
surge margin, a decrease in efficiency and pressure rise at a given mass flow, and can lead to an amplification of vibrational modes that can lead to HCF. In the case of stall margin and vibrations, certain kinds of distortion patterns have been shown to dampen vibration modes.

\subsubsection{Definitions of Inlet Distortion}

Static and total pressure distortion are the most common forms of flow distortion that are studied in research, and they are the subject of most papers covering the topic of centrifugal inlet distortion. A total pressure distortion consists of any variation in total pressure along a plane perpendicular to the direction of fluid flow. Total pressure distortion, for example, could result from a flow disturbance (strut, vane, etc.) that would create a low velocity wake surrounded by higher velocity fluid. The non-isentropic nature of such a flow disturbance will ultimately lead to a pressure loss, and it will be of a non-uniform nature across the total inlet flow.

Due to the need for efficient packaging of engine hardware in aerospace applications, the requirement for specialized, non-uniform, complex inlets may arise. A common inlet design is similar to the advanced radial inlet studied by Kim and Koch (2004), Figure 1.2, where flow from a circular port is wrapped around the impeller inlet, in a scrolllike fashion, resulting in a complex and distorted pressure flow field at the impeller inlet. These types of inlets are popular with space-constrained compressor systems. 


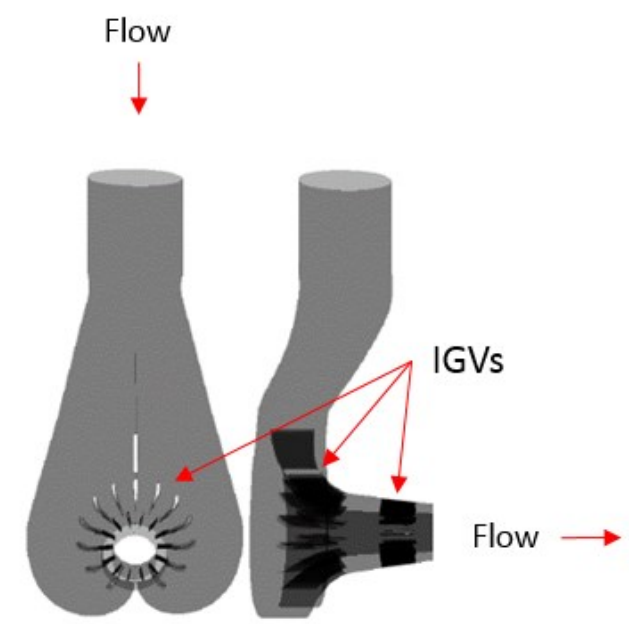

Figure 1.2: Design of radial inlet for a centrifugal compressor with IGVs, Kim and Koch (2004).

Other sources of pressure distortion can arise from support struts that are placed in the inlet flow field, studied by Kammerer and Abhari (2010), boundary layer growth along inlet walls, flow separation at bends, or inlet guide vanes. A popular subject of study is pressure distortion generated from a bend in a circular pipe. This introduces several secondary flow mechanisms that result in a non-uniform flow at the exit of the bend.

A common way of defining temperature and pressure distortion is by referring to the number of "lobes" of distortion present in the flow. This stems from the use of screens that block the flow in a circumferential pattern, with the number of lobes defining the number of symmetric sections of distortion. An example can be found from Zemp et al. (2010) in Figure 1.3, where a five-lobe total pressure distortion was generated. 


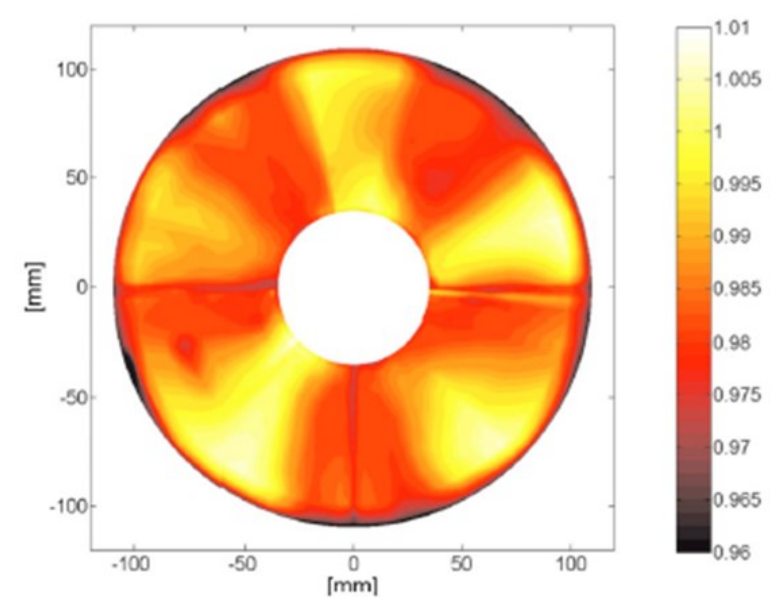

Figure 1.3: Inlet total pressure distortion, normalized by upstream pressure rakes, Zemp, et al. (2010).

Temperature distortion studies in modern research literature are much less common, but this does not discount the importance of studying its effects on compressor performance. Cousins et al. (1994) cite that temperature effects on compressor performance, in particular surge and stall margin, can outweigh the effects of pressure distortion, much to the contrary of researchers who claim the opposite. Temperature distortion decreased surge margin by nearly $10 \%$, and similar results were seen with combined pressure and temperature distortion. The largest degradation in stability margin was seen where the existing stability margin was largest, and was strongly dependent on simulated altitude conditions during testing.

Flow angle distortion is usually defined by any variation in flow angle away from the meridional, or axial direction of the compressor. This is often a subject of concern when a significant bend, or change in flow direction exists. Engeda et al. (2003) showed that flow angle distortion changes the incidence angle at the impeller inlet, causing efficiency loss 
and total pressure loss as well as vibrational problems, depending on the type and intensity of the distortion.

Distortion is characterized by two categories - radial and circumferential distortion, with radial distortion being further subdivided into hub and tip distortion. Beyond these categories, the amount of distortion and method of generating distortion varies significantly between researchers, which can make it difficult to directly compare results. This is due to the fact that researchers may use different combinations of distortion, such as a combination of pressure and flow angle distortion, at different magnitudes and quantified through various means and averaging schemes.

Engeda et al. (2003) created inlet distortion using a 90-degree circular bend upstream of the compressor, as did Kim et al. (2001), Kang et al. (2012), and Engeda et al. (2003). Zemp et al. (2010) used different mesh screens to generate a lobed distortion patterns. Ariga et al. (1987) used honeycomb patterns to create distortion patterns, not in a lobe-type pattern, but hub and tip distortion, as well as a half-circumferential distortion pattern.

\subsubsection{Inlet Distortion Effects on Compressor Performance}

The most evident effects of inlet distortion are in the Total Pressure Ratio (TPR) and efficiency of a compressor stage, but the causes of such effects are very complex. Cousins et al. (1994) notes that the aerodynamic time constant for a compressor blade is a function of blade chord, and that a longer time constant of a centrifugal compressor is significantly longer than that of most axial machines, which makes them more tolerant to distortion effects than axial compressors. This is affirmed in research by Zemp et al. (2010) and Kammerer and Abhari (2010) showing that by the time the airflow reaches the exit of 
the impeller blade chord, the effects of inlet distortion are much less significant than at the inlet. Cumpsty (2004) explains that for an axial compressor, circumferential distortion has the most significant effect on performance, mostly in the reduction of surge margin. There is usually a limit for the swept circumferential angle of inlet distortion at which point additional distortion angle causes almost no change in compressor surge. Research into this effect on centrifugal compressors was not found, but results from Ariga et al. (1987) show that at higher mass flow rates, hub distortion as opposed to circumferential distortion has the most significant impact on performance, whereas circumferential distortion can sometimes even have beneficial effects, such as an increase in stall margin. Generally all research points to the fact that effects of distortion increase in severity with a decrease in flow coefficient.

Researchers tend to agree that as the magnitude of total pressure distortion increases, there will be a decrease in the overall pressure ratio for a centrifugal compressor at a given operating speed and, consequently, a loss in efficiency. A major part of the loss is usually driven from a change in incidence angle at the impeller inlet, as described by Gancedo et al. (2012). If the relative flow angle coming into the impeller does not coincide perfectly with the blade angle, the kinetic energy of the fluid (in the tangential direction) will be wasted and result in head loss and efficiency loss at design point conditions. This can arise equally from steady or quasi-steady distortion.

In the specific case of inlet distortion generated by a sharp bend in the inlet flow coming into a compressor, a pressure gradient normal to the streamline curvature is created which generates a secondary flow pattern that results in pressure and flow angle distortion, as studied by Kim and Koch (2004). 
Research done by Cousins et al. (1994) claims that total temperature distortion has a greater effect on compressor stability than total pressure distortion. They operated a twostage centrifugal compressor that was outfitted with a hydrogen gas burner to create temperature distortion patterns and screens to create pressure distortions. The surge line moves toward higher flow rates at higher rotational speeds but interestingly moves toward lower flow rates for lower rotational speeds. Although combustion by-products from the hydrogen flame will alter the gas properties of the air flowing through the compressor, the author made no note concerning this phenomenon.

\subsubsection{Aeromechanic Response to Inlet Distortion}

Inlet distortion, either steady or quasi-steady, can also be responsible for the amplification or dampening of vibrational modes in the impeller. Due to the nature of research that encompasses vibrational response, ultra-high response electronics are required to characterize the nature of aerodynamic blade forcing functions that develop due to distorted inlet flow. This requires expensive, state-of-the-art equipment, making this type of research relatively new and quickly developing field of research.

Kammerer and Abhari (2010) instrumented a centrifugal compressor with highfrequency response pressure transducers and strain gauges on the blades of the impeller to measure the vibrational response as well as aerodynamic forcing functions. Distortion screens were installed to create a 5 or 6-lobe circumferential inlet distortion. By measuring the unsteady pressure fluctuations directly on the impeller, they found that pressure side fluctuations tend to be larger than suction side fluctuations, which was confirmed by CFD research by Zemp et al. (2010). These pressure fluctuations were mostly driven by changes 
in flow area between blades, and this driving force outweighed contributions from inlet distortion.

Kammerer and Abhari (2010) showed that with distortion screens installed on a centrifugal compressor, higher engine orders are excited (EO 5 in particular) at offresonance conditions, and engine orders already present with no distortion are amplified. They showed that during resonance, blade displacement and pressure measurements can be related - as the pressure side of the blade moves in the direction to lower the flow area between blades, it encounters a lower pressure, whereas the suction side experiences a higher pressure. These strong pressure fluctuations were caused by the acceleration and deceleration of fluid flow between the blades by means of an increase or decrease in flow area. Aerodynamic work measurements indicate pressure fluctuations due to blade movement outweigh the pressure fluctuations due to inlet distortion. This finding is consistent with findings from Lawless and Fleeter (1994) where during events where inlet distortion was generated in an attempt to suppress the first engine order, but the third engine order was amplified. These amplifications increased with an increase in engine speed. CFD research by Zemp et al. (2010) based off of the same compressor setup indicate that flow separation caused by incidence changes from flow distortion considerably altered amplitude and phase angle of the unsteady pressure fluctuations, which helps explain the shift in engine order amplitudes. In similar research, Fridh et al. (2013) showed that turbines under 5 different configurations of partial admission "inlet" distortion saw significant increases in the amplitude of low-engine order forced response magnitudes over no inlet distortion, especially in low engine order frequencies. 
Rodgers (1988) showed that inlet distortion decreased impeller stage efficiency by $1.8 \%$, but diffuser efficiency remained essentially unchanged, indicating that the effects of distortion on impeller performance becomes less significant when advancing along the meridional direction toward the diffuser.

\section{$1.4 \quad$ Research Objectives}

The purpose of this research can be divided into two separate parts. The first part of the research is focused primarily around the instrumentation and set up of a test cell in the Purdue High Speed Compressor Research Laboratory to accommodate research in a centrifugal research compressor which will be instrumented with Kulite pressure transducers, total pressure and temperature rakes, strain gauges, capacitance probes, and an LDV measurement system. The centrifugal compressor research will investigate the effects of inlet distortion on forced response, overall performance, and will also focus on impeller/diffuser interactions by adjusting the gap between the two components. Additional testing of an APU-style inlet will also be conducted before the delivery of the centrifugal compressor. This inlet was modified to incorporate optical access for a LDV measurement systems to characterize the flow velocity. This will allow for the characterization of the inlet flow field to the compressor, validating CFD simulations before the arrival of the compressor. A gas ejector system was constructed to provide similar airflow to the inlet that it will experience during normal operation with the centrifugal compressor. Also, a large electronics instrumentation suite was designed and constructed that can accommodate hundreds of steady pressure and temperature channels 
for compressor testing, as well as the amplification and analog to digital conversion of fast response instrumentation.

Secondly, this research focuses on a different compressor, the Purdue 3-Stage axial compressor, and the flow field around an embedded stage within the compressor and how it is responsible for creating a forced response vibratory condition. All rotor blades in the 3-stage compressor are manufactured in a bladed-disk or "blisk" design, meaning they are all part of one solid machined assembly. While this can create a lighter, easier to build rotor assembly, blisk designs have been known to accentuate the amplitude response of bladed rotors. The response characteristics of the R2 assembly were measured with an NSMS tip timing system, and the upstream vortical forcing as well as the downstream potential forcing were measured with thermal anemometry and stagnation pressure rakes to quantify the forcing function that drives the R2 response. High response pressure transducers were used in an attempt to detect $\mathrm{R} 2$ forced response vibration with a pressure transducer to develop a new method of detecting resonant vibration in a compressor. These data were acquired to create a database for aerodynamic code validation and to advance model development. Additionally, the response characteristics of all rotor and stator assemblies were extensively measured to provide a complete map of safe operating speeds and conditions for the Purdue 3-stage axial compressor. 


\section{CHAPTER 2. PREPARATIONS FOR CENTRIFUGAL COMPRESSOR RESEARCH}

\subsection{Motivation and Requirements}

This chapter describes the design of experiments and supporting systems needed to run the Honeywell Single Stage Centrifugal Compressor (SSCC). This is a new research program started in 2011 and funded by Honeywell. Significant planning and resource investments have taken place in the past 2 years with the estimated $1^{\text {st }}$ spin to occur at the end of 2014. This project will focus on understanding the effect that different inlet geometries have on the performance and aeromechanical response of a centrifugal compressor, with heavy emphasis on acquiring and processing unsteady data. The two different compressor inlets include an Auxiliary Power Unit (APU) style inlet, characterized by a vaned diffuser attached to a 90 degree elbow that exits to the compressor, and an axisymmetric cylindrical inlet. Before the arrival of the compressor, and while the test cell was being prepared for its installation, the test cell would be modified for flow characterization studies of the APU-style inlet.

To investigate the flow field of an APU-style inlet for a centrifugal compressor, air suction capability similar to the Honeywell SSCC compressor was installed. The purpose of the experimental setup is to characterize the flow field inside a rectangular duct undergoing a 90-degree bend using Laser Doppler Velocimetry (LDV) equipment. 
The main motivation for the inlet characterization was evidence previously acquired at Honeywell of an unexpected forced response measured in the compressor when operated with this inlet. The 3-component velocity within the APU style inlet will be compared with Honeywell CFD simulations to better understand the flow field and improve understanding of necessary elements of the model to predict this situation in future builds. Figure 2.1 shows a model of the test cell with the proposed compressor, exhaust plenum, APU inlet, gearbox, and $\mathrm{ABB}$ motor.

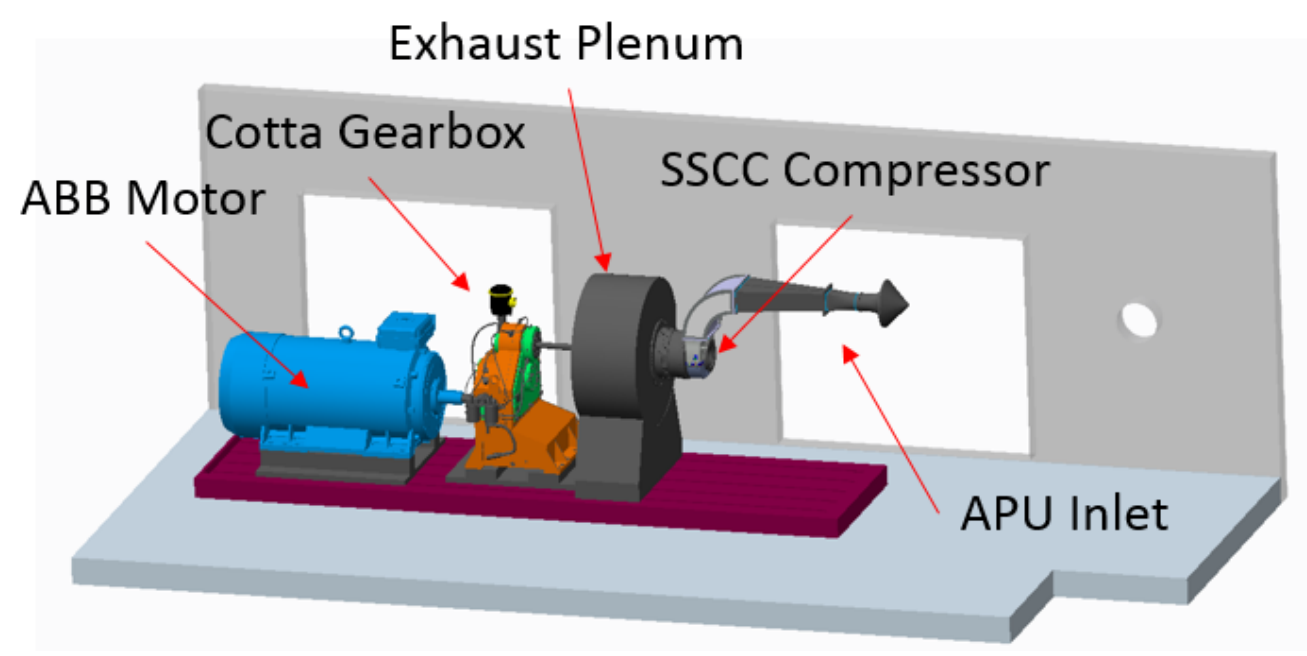

Figure 2.1: Proposed cell layout with ABB motor, gearbox, compressor, and APU inlet.

\subsection{Inlet Flow Setup Design}

The APU inlet will operate at a flow rate of $5.5 \mathrm{lbm} / \mathrm{s}$. Large areas of optical access were added to the Honeywell-supplied APU hardware so that LDV data could be obtained. It was important to take into consideration the focal length of the LDV equipment and the amount of space required in order to obtain data throughout the entire elbow. The suction applied to the inlet should allow long duration runs to enable LDV traverses of large flow regions. All data would be taken at the air mass flow rate equal to $100 \%$ operating speed 
of the compressor. However, fine tuning of the flow rate would be required to compensate for changes in air density as a result of ambient pressure and temperature conditions, as well as changes in the physical properties of the motive air stream.

To fulfill the requirements for the suction flow to the facility, a Schutte and Koerting air ejector, also known as a jet pump, was installed outside of the test cell, as seen in Figure 2.2.

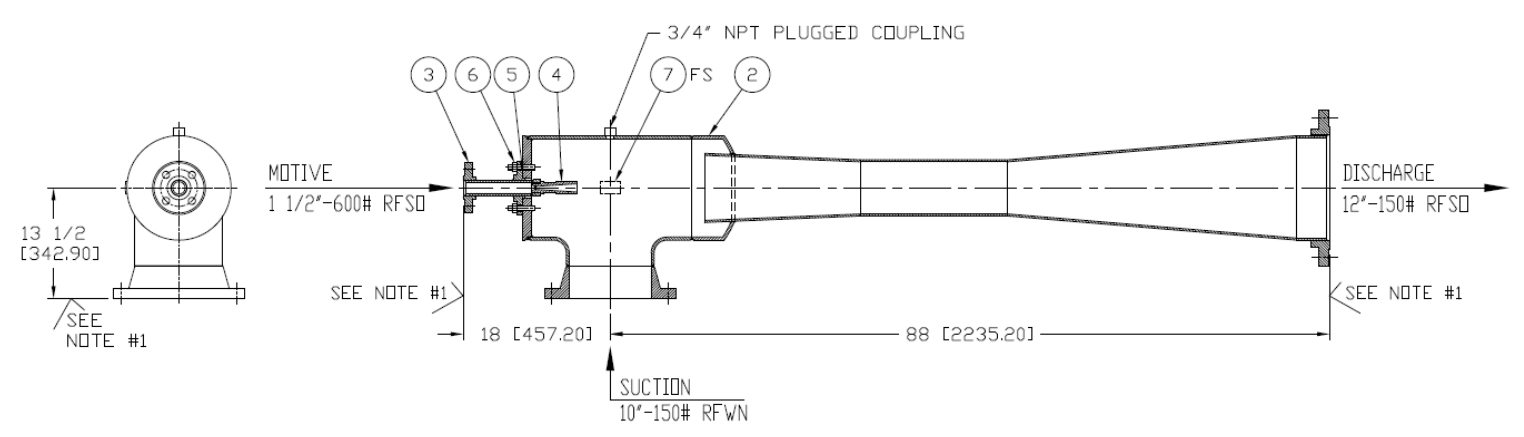

Figure 2.2: Engineering drawing of Schutte and Koerting Air Ejector.

The ejector works by entraining a "suction" airflow through the use of a "motive" airflow. The pressure in a motive airstream is converted to kinetic energy through the use of a venturi nozzle, in this case a supersonic nozzle, and this high velocity airstream creates a low pressure zone within the body of the ejector that creates a suction enabling air flow through the system. Viscous mixing within the two airstreams also contributes a portion of the entrained massflow. With this particular ejector, the entrained suction mass flow rate is 2.5 times that of the motive air flow, which creates essentially a high mass flow rate air pump with no moving parts. The kinetic energy of the two mixed airstreams is then converted partially back to pressure through the use of a subsonic diffuser before being exhausted to ambient air. By varying the pressure of the incoming motive flow and, 
therefore, the motive mass flow rate, the amount of suction mass flow rate and suction pressure the ejector creates can be controlled.

The Zucrow high pressure air system supplies the required motive air flow to the ejector. The ejector was designed to operate at 500 psi with $2.2 \mathrm{lbm} / \mathrm{s}$ second of motive airflow (1997 SCFM) in all ambient temperature conditions. The high pressure air system can deliver anywhere from 600 to 2,200 psi of air pressure, depending on the amount of air pumped into the high pressure holding tanks. At $2.2 \mathrm{lbm} / \mathrm{s}$ of motive flow, the ejector was designed to provide $5.5 \mathrm{lbm} / \mathrm{s}$ of suction flow at $12.2 \mathrm{psia}$. The motive airflow is controlled by a TESCOM dome-loaded 26-1200 regulator that requires a 26-2000 pilot regulator to drive the dome, which itself is controlled by an electronic ER-3000 regulator. The ER3000 regulator is controlled through LabView and the built-in TESCOM PID control software to provide the needed motive pressure for a desired suction mass flow rate, which is measured by a Honeywell-calibrated bellmouth at the entrance of the APU inlet. All air flowing to the main 26-1200 regulator and the ER-3000 is filtered by Norman cartridge air filters. The pressure upstream and downstream of the regulator is measured to calculate overall remaining run time based on the pressure in the high pressure air tanks and provide the final feedback loop control for the ER-3000 PID control system. Figure 2.3 shows the flow layout that is used to control the motive air stream. 


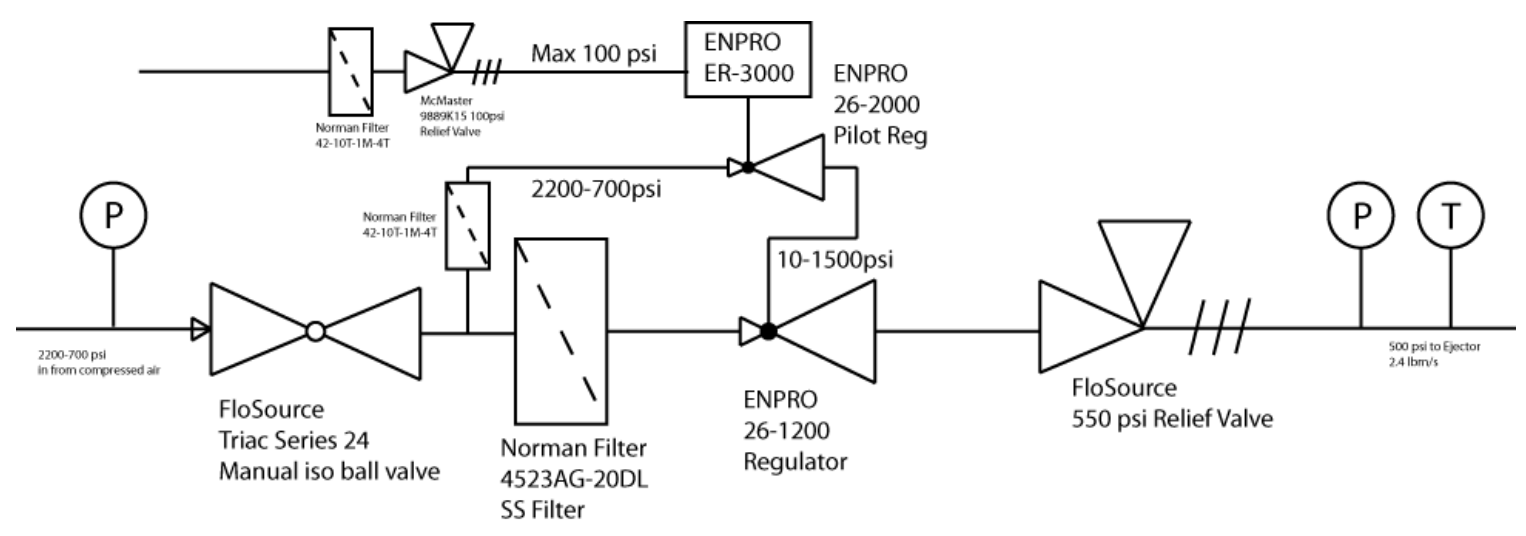

Figure 2.3: Ejector Flow Schematic

Since the ENPRO 26-1200 regulator has a $C_{v}$ of 12 , the motive air system could still deliver the required 1997 SCFM of airflow at 500 psi with a pressure loss of approximately $50 \mathrm{psig}$, giving the system the ability to still function at the lowest tank pressure of $600 \mathrm{psi}$, Figure 2.4. Taken from the Swagelok Valve Sizing Technical Bulletin, MS-06-84-E, the required $C_{v}$ was calculated two ways depending on whether or not the valve was choked. The parameter $N_{2}$ is a constant set to 22.67 to balance out units, $G_{g}$ is the gas specific gravity, $q$ is flow rate, $T_{1}$ and $p_{1}$ are upstream pressure and temperature, and $p_{2}$ is the downstream pressure. By combining Eqn. 2-1 Eqn. 2-2, Figure 2.4 is generated for the ejector system, showing flow rate conditions are met even at a pressure drop of less than 100 psi.

$$
\begin{array}{cc}
C_{v}=\frac{q}{N_{2} p_{1}\left(1-\frac{2\left(p_{2}-p_{1}\right)}{3 p_{1}}\right)} \sqrt{\frac{p_{1} G_{g} T_{1}}{\left(p_{2}-p_{1}\right)}} \quad p_{2}>0.5 p_{1} \quad \text { Eqn. 2-1 } \\
C_{v}=\frac{q \sqrt{G_{g} T_{1}}}{0.471 N_{2} p_{1}} \quad p_{2}<0.5 p_{1} & \text { Eqn. 2-2 }
\end{array}
$$




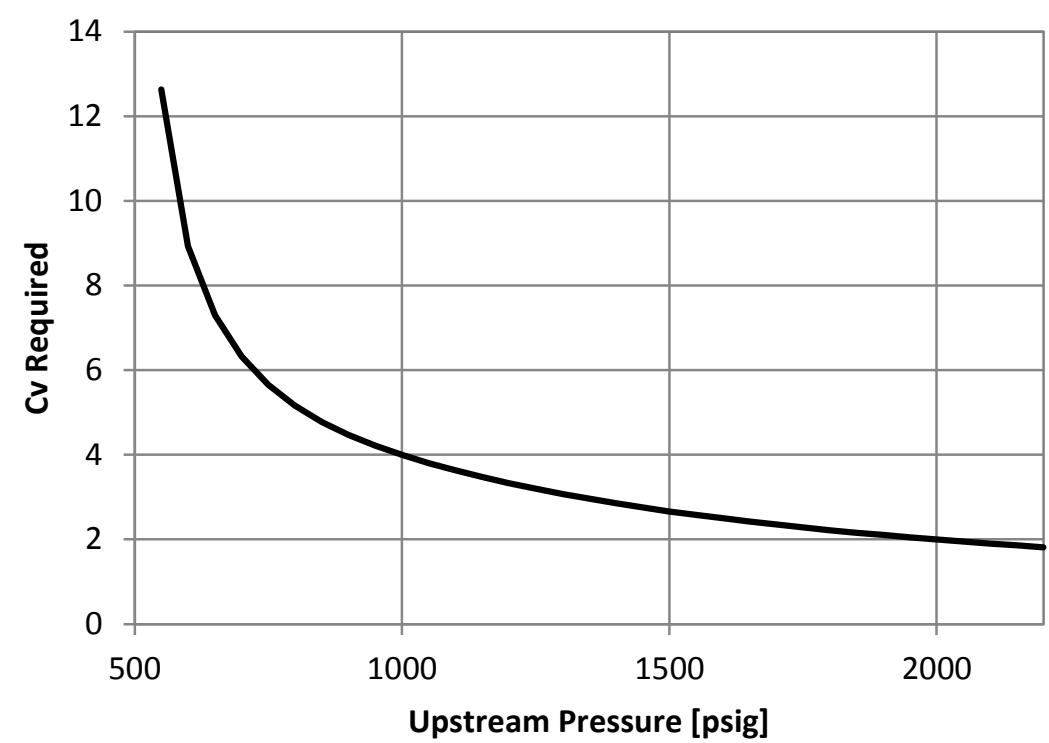

Figure 2.4: Required $C_{v}$ for different pressures to maintain ejector motive flow.

A manual isolation ball valve is used to cut off air to the entire flow circuit before and after the ejector is used. There is currently a 675 psi burst disc installed upstream of the ejector inlet that will burst in case of a failure from either of the two regulators. The main body of the ejector is rated for pressures up to 1,000 psi.

The mounting system for the APU inlet and the ejector went through several design iterations, and the final choice of having the ejector outside of the test cell was chosen to provide enough distance between the APU inlet and the ejector to avoid any potential field effects as a result of downstream obstructions or bends in the flow ducting. Flow entering the bellmouth, likewise, was required to be uniform, and thus, the APU inlet was mounted horizontally, with the two LDV windows facing the floor and the ceiling. A 47" long rectangular duct was installed downstream of the inlet system to provide uniform suction flow before connecting to a rectangular-to-circular transitional duct. This transition piece connects to a 10 " pipe that bolts to the suction inlet of the ejector. The combined motive 
and suction flows are then vented outdoors. Figure 2.5 shows a top down view of the test cell with the flow system installed, including the SSCC gearbox and ABB motor.

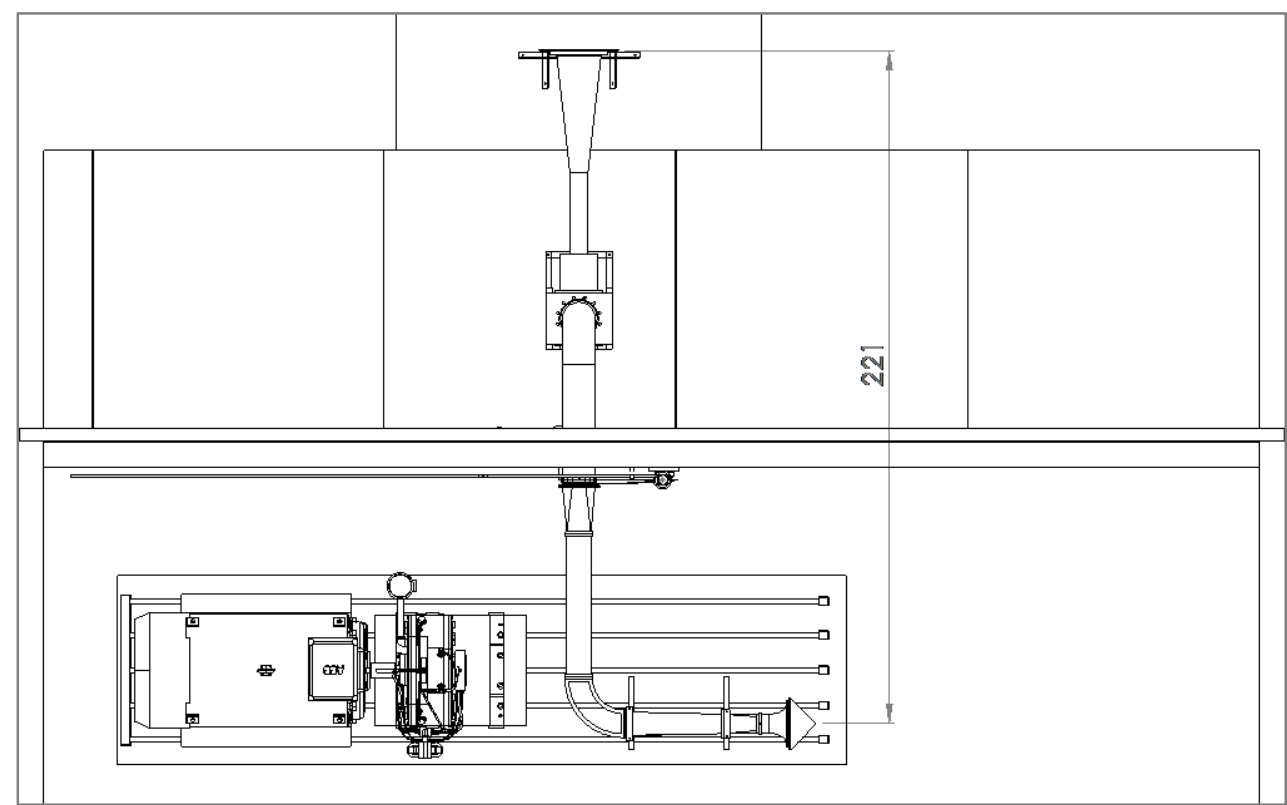

Figure 2.5: Test cell with Ejector setup, main dimension in inches.

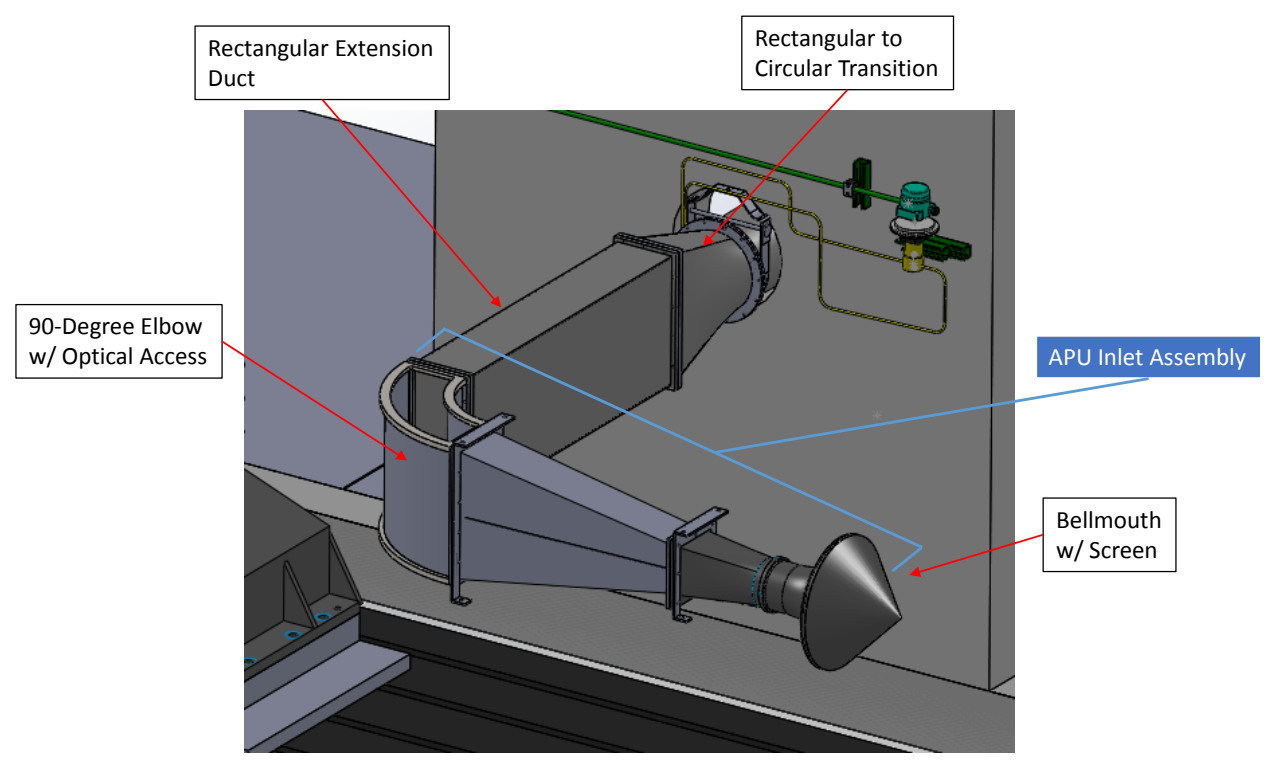

Figure 2.6: APU Inlet installed in test cell in orientation for Ejector flow with LDV instrumentation access. 


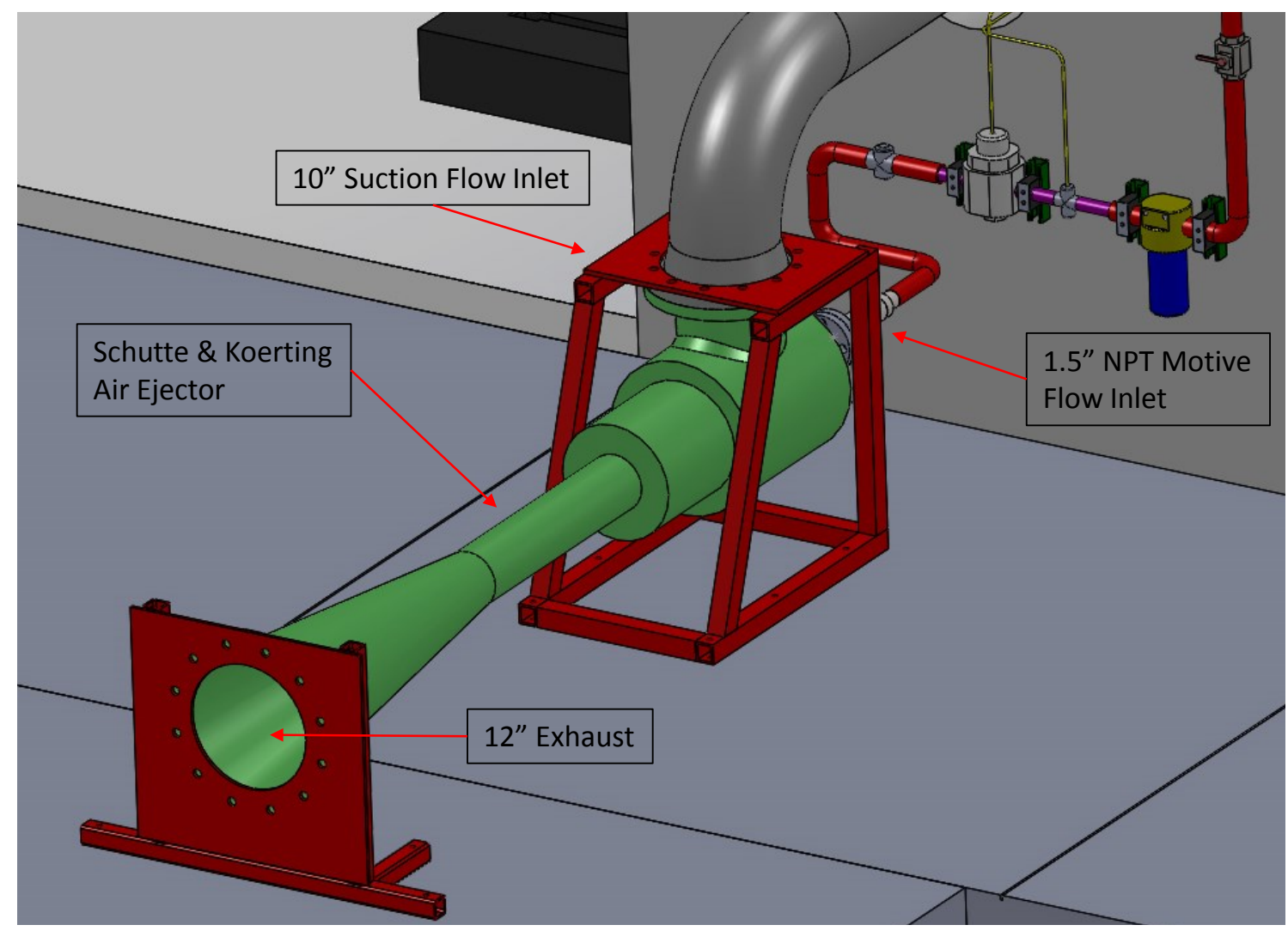

Figure 2.7: Outdoor ejector installation model, showing motive air plumbing.

Figure 2.6 and Figure 2.7 are solid models of the inside and outside of the test cell, respectively. Figure 2.6 shows the APU inlet with the optical access windows, and Figure 2.7 shows the ejector mounted to a welded steel beam fixture to secure it to the concrete outdoors. The motive air enters the back of the ejector, and it exhausts outwards, perpendicular to the building.

\subsection{Elbow Design}

To acquire LDV measurements on the APU inlet flow, the inlet system was modified to provide optical access, specifically at the location of the 90 degree elbow. The new elbow had to provide the same internal flow path geometry as the original stainless steel elbow that was provided by Honeywell. The elbow needed scratch-resistant windows that 
would handle the vibrations and pressure loading from normal compressor operation as well as loads generated by stall and surge of the compressor, since this elbow design would eventually be used to acquire LDV data when compressor testing began. A design requirement withstanding an ultimate load of 20 psig overpressure acting from the inside on all wetted surfaces was provided by Honeywell.

A removable window design was considered to facilitate cleaning through the use of fasteners around the perimeter of the optical window. However, this was difficult to manufacture and difficult to maintain a consistent inner surface that would match the same profile of the Honeywell elbow.

The final design consisted of a window that was bonded in place through the use of $3 \mathrm{M} ®$ VHB tape that would secure the window to a female flange feature on the inner side of two outer frames. The flange would hold the window firmly in place, take the loads of an overpressure from surge, and bear the atmospheric pressure loads during operation when the air inside the duct would have a negative gauge pressure. This design was very simple, required only two permanent seals (around the window edges), and required very few parts to construct, and thus, was a cost effective solution.

Figure 2.8 shows a cutaway of the elbow halfway through the bend, where the window mounting flange design is displayed. Figure 2.9 shows the completed assembly as designed. 


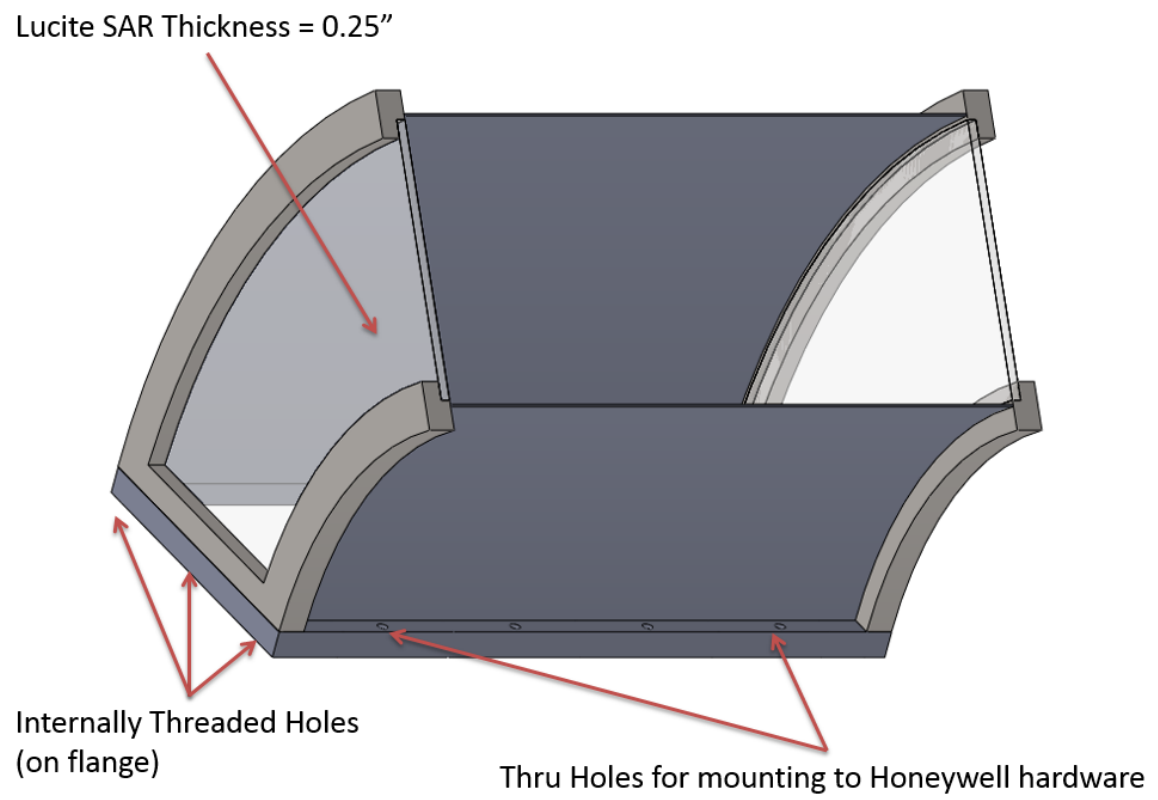

Figure 2.8: Section view of redesigned elbow, illustrating Lucite SAR windows for LDV.

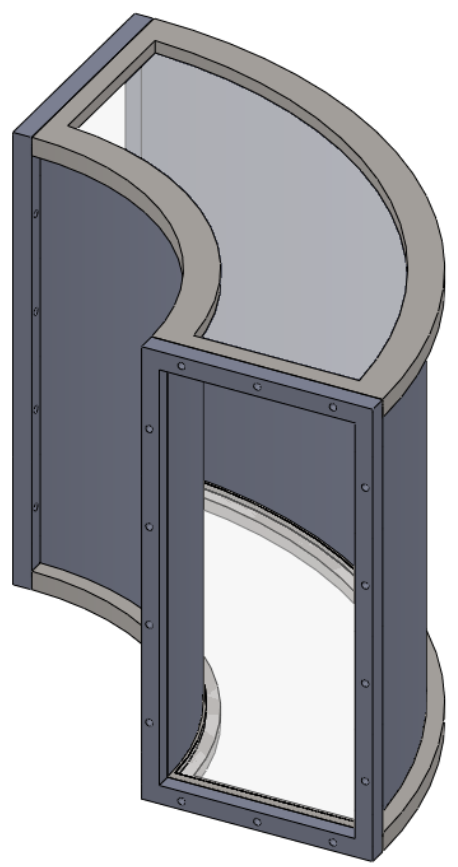

Figure 2.9: The final elbow design, with .062" thick aluminum sheet walls and LUCITE SAR acrylic windows for LDV optical access. 
The windows were constructed of Lucite SAR (Super Abrasion Resistant) acrylic. This is a material often used for basketball backboards, racquetball courts, and aquariums, and it is noted for its superior abrasion resistance. This was recommended by Dantec Dynamics, the manufacturer of the LDV system.

ANSYS 14.0 was used to simulate the overpressure loads and ensure that the elbow duct would not experience excessive deformation or yielding throughout operation. Generic 316 Stainless Steel material properties in ANSYS were used for the metal framing, and average acrylic properties (Young's Modulus) were used for the Lucite material because of a lack of published data on Lucite. However, published $F_{\text {sy }}$ values from Lucite manufacturer specs were used to calculate the ultimate yield margins. The mesh size was limited to 200,000 nodes per the ANSYS educational license, so 2-way symmetry (cutting the elbow at the $45^{\circ}$ part of the bend, and halfway along the height of the duct), was used to allow increased mesh density. It was solved with 7 iterations over 1 second of simulation time. Maximum mesh element size was 0.135 ", with a medium relevance setting and hex dominant method forced along the entire part. This resulted in 167,804 nodes with 30,057 elements, with an average mesh quality of 0.803 .

The simulation was set up with frictionless supports on the top and inner radius. There was a 20 psi overpressure load on all inner surfaces, and a proportional blowoff load of $1,317 \mathrm{lb}_{\mathrm{f}}$ applied to the bolt holes on the flange, with two symmetry planes to hold the rest of the assembly in place. Bonded connections for all parts were used to simulate welds and the connection between the window and its inset mounting flange.

The results show that the stresses in the acrylic windows are very low, and the yield margin is met. This was with an overly conservative bonded connection that was probably 
much stiffer than the VHB tape. The adhesive tape is expected to absorb some of the stress related to the deformation of the metal flange, resulting in a predicted higher stress than will occur in actual operation. The stresses in the 316SS exceed yield strength in the corners for only one element width. This is most likely due to the nature of the mesh grid and the use of a linear solver. The parts on the design that are indicating failure are of the same dimensions as the elbow that Honeywell supplied Purdue which has already seen extensive use with no catastrophic failures. Therefore, these locally yielding elements were ignored. Should yielding occur in real-life operation, this local yielding will occur and lower overall stresses of the part, preventing ultimate tensile failure. Figure 2.10 shows the equivalent von-Mises stress in the metallic components, and the max stress occurs at the corner marked with the red indicator tag.

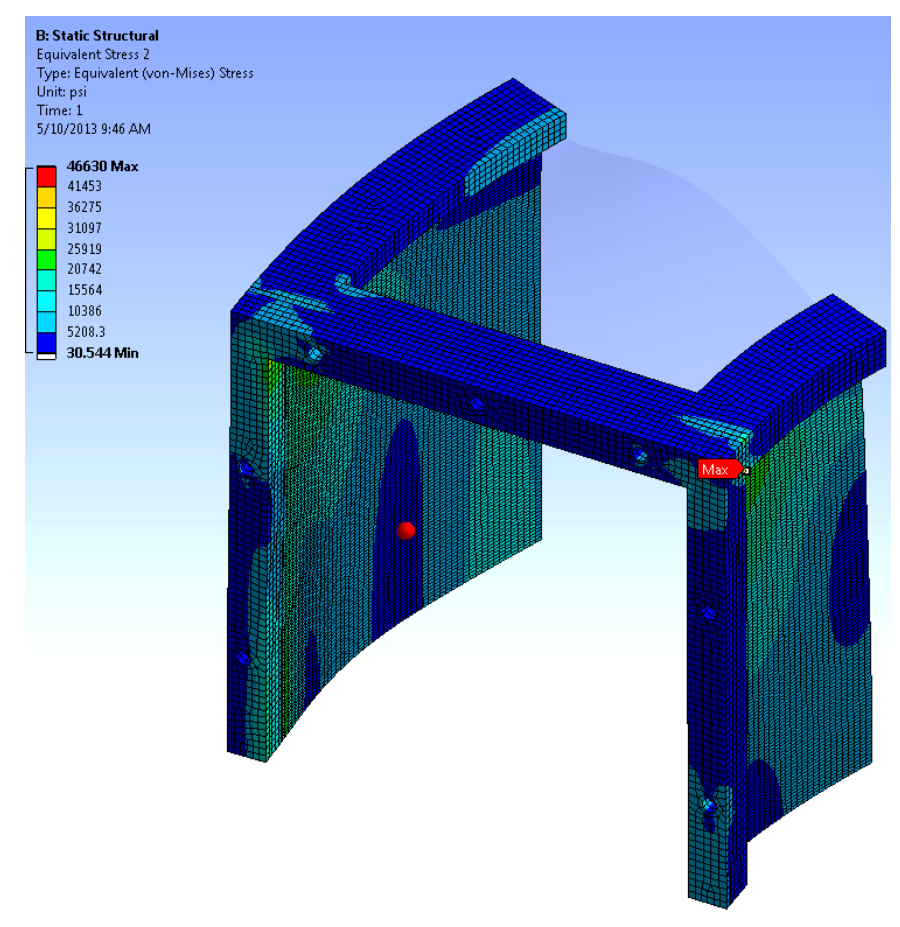

Figure 2.10: Static structural stresses in metallic components. Yield allowable is $32.3 \mathrm{ksi}$. 


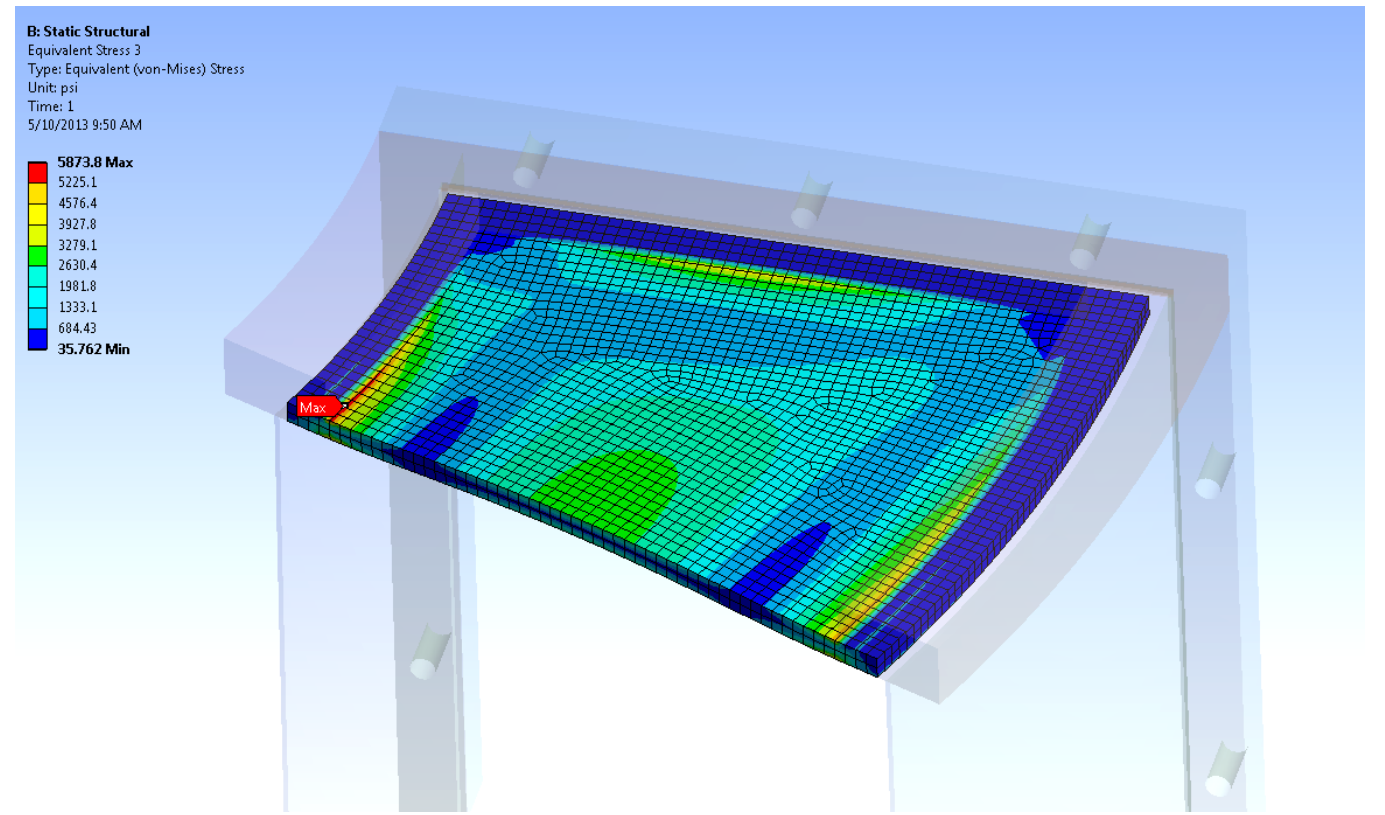

Figure 2.11: Static structural stresses as seen by top of acrylic window, where max stress occurs.
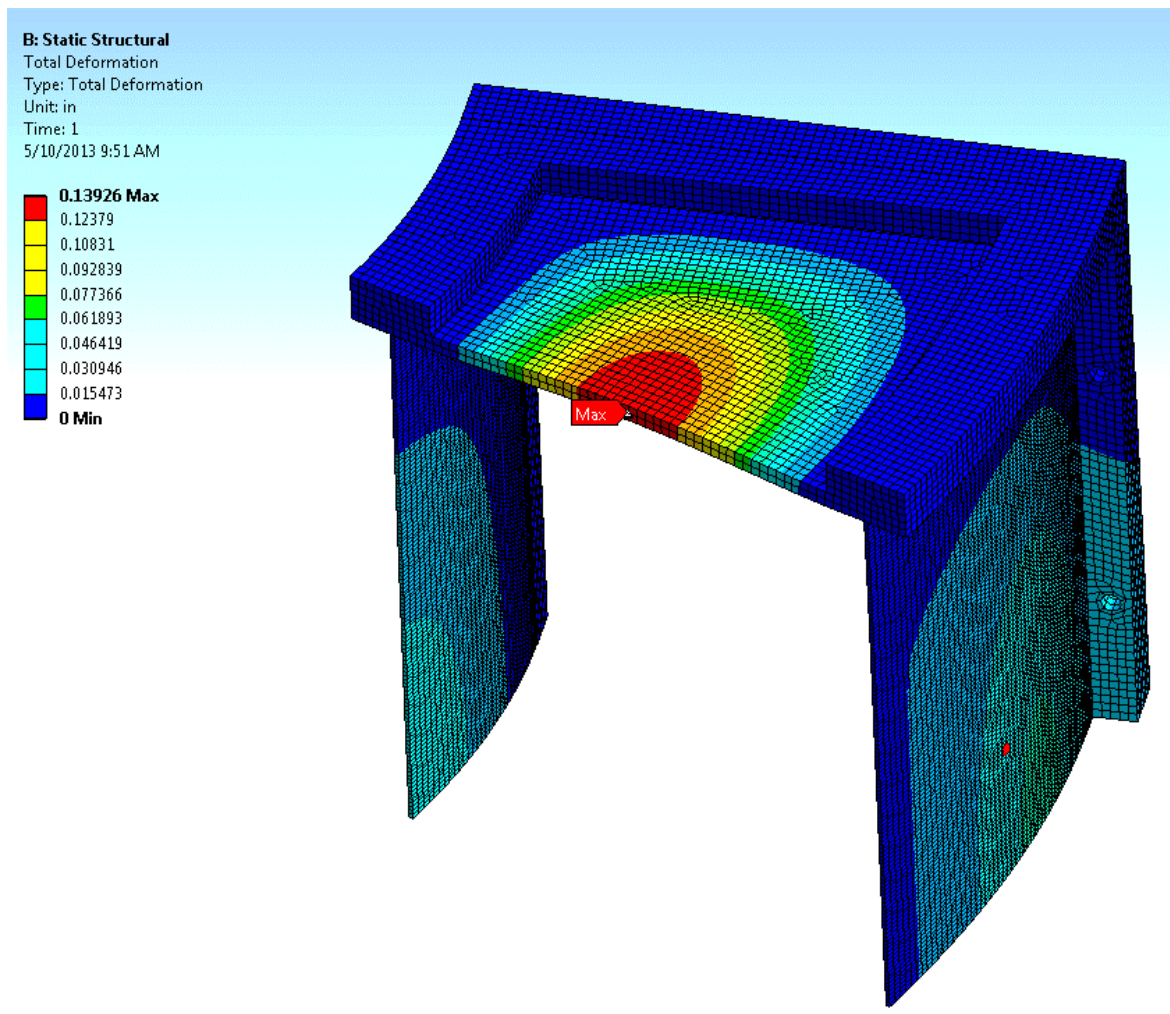

Figure 2.12: Part deformations, maximum seen in window of 0.139 ” at max pressure. 
Figure 2.11 shows the maximum stresses in the window alone, where the maximum stress occurs where the lip of the steel frame holds the window in place, and another large stress area occurs in the center of the window. Due to the low modulus of the window, it is allowed to deform significantly in comparison to the steel, Figure 2.12, keeping stress values below yield allowables. Table 2-1 shows the material properties used for the steel and the window. Table 2-2 shows the max stress, yield, and ultimate margins based on ANSYS simulations and the input material properties.

Table 2-1: Material properties used in elbow simulation.

\begin{tabular}{|c|c|c|c|c|c|}
\hline & Density & Fsy & Fsu & Young's Modulus & Poisson's Ratio \\
\hline & $\mathrm{lb} / \mathrm{in}^{3}$ & $\mathrm{psi}$ & $\mathrm{psi}$ & $\mathrm{psi}$ & \\
\hline Acrylic (Lucite SAR) & 0.042 & 6820 & N/A & $4.12 \mathrm{E}+05$ & 0.37 \\
\hline 316SS Annealed & 0.279 & 36260 & 66720 & $2.90 \mathrm{E}+07$ & 0.30 \\
\hline
\end{tabular}

Table 2-2: Margins in Lucite and Stainless from overpressure simulation.

\begin{tabular}{|c|c|c|c|}
\hline Margins & Max Stress (psi) & Yield Margin & Ultimate Margin \\
\hline Acrylic Lucite & 5873 & 1.16 & N/A \\
\hline 316SS 1 element from corners & 46630 & 0.78 & 1.43 \\
\hline 316SS $>$ 1 element from corners & 19882 & 1.82 & 3.36 \\
\hline 316SS Sheet & 31414 & 1.15 & 2.12 \\
\hline
\end{tabular}

\section{$2.4 \quad$ Test Cell Instrumentation}

This section presents the instrumentation that is used for both the SSCC compressor as well as the APU/ejector layout. Outside of the concrete test cell, a control station will be used to monitor the gearbox, motor, and compressor. It will also be used to control and monitor instrumentation as well as acquire fast response data. The layout of the instrumentation system is such that there are two separate data acquisition systems, one for 
performance measurements and one for health monitoring. The health monitoring system will be responsible for acquiring all data pertaining to the operation of the gearbox and monitoring and control of the compressor. The compressor performance system is comprised of similar hardware and a separate computer, with the addition of high-speed data acquisition hardware and amplifiers.

The compressor performance DAQ system consists of Agilent multiplexer-enabled TC readers, 256 channels of Scanivalve dry pneumatic pressure channels, backup power supplies, a PXI chassis for analog to digital conversion, and Precision Filter amplifiers used for strain gauges and Kulite pressure transducers. The Agilent TC readers are capable of accepting any type of thermocouple. It will also consist of two Mensor pressure transducers, used for the acquisition of pressures for calculating compressor inlet mass flow rate.

The health monitoring system will be comprised of a similar computer, backup power supply, and Agilent multiplexer, but it will have its own PXI chassis with a slower analog-to-digital converter, and it will control all cameras that will be used to monitor the test cell. The PXI chassis will be responsible for monitoring oil, air, and gearbox system temperatures and pressures, as well as output voltages for control systems. The health monitoring computer will also handle all TESCOM controls for the air system as well as read in pressures from the Rosemount pressure transducers used to measure venturi mass flow rates of the outgoing air bleeds from the compressor. Table 2-3 shows a detailed list of all instrumentation:

Table 2-3: List of major data acquisition equipment purchased for Honeywell research.

\begin{tabular}{|c|c|c|c|c|}
\hline Manufacturer & Instrumentation & $\begin{array}{c}\text { \# of } \\
\text { Ch }\end{array}$ & Qty & Description \\
\hline Agilent & $33220 \mathrm{~A}$ & 1 & 1 & Waveform Generator \\
\hline Agilent & $34410 \mathrm{~A}$ & 1 & 1 & Digital Multimeter \\
\hline
\end{tabular}




\begin{tabular}{|c|c|c|c|c|}
\hline Precision Filter & PF 28118 & 8 & 4 & Unsteady Full Bridge Amp \\
\hline $\begin{array}{c}\text { National } \\
\text { Instruments }\end{array}$ & NI PXIe 6356 & 8 & 1 & $1.2 \mathrm{MS} / \mathrm{s}$ per ch DAQ Card \\
\hline $\begin{array}{c}\text { National } \\
\text { Instruments }\end{array}$ & NI PXIe 6358 & 16 & 2 & $1.2 \mathrm{MS} / \mathrm{s}$ per ch DAQ Card \\
\hline Scanivalve & $\begin{array}{c}\text { DSA3016 100 } \\
\text { PSID }\end{array}$ & 16 & 10 & Steady Pressure Transducer \\
\hline Scanivalve & DSA3016 5 PSID & 16 & 4 & Steady Pressure Transducer \\
\hline Scanivalve & $\begin{array}{c}\text { DSA3016 2.5 } \\
\text { PSID }\end{array}$ & 16 & 2 & Steady Pressure Transducer \\
\hline Agilent & 34980 & 40 & 3 & TC Multiplexer/Reader \\
\hline $\begin{array}{c}\text { National } \\
\text { Instruments }\end{array}$ & PXIe 6224 & 32 & 1 & Health Monitoring DAQ \\
\hline Mensor & 61101.5 PSID & 1 & 1 & $\begin{array}{c}0.01 \% \text { Accuracy P } \\
\text { Transducer }\end{array}$ \\
\hline Mensor & $611013-15$ PSIA & 1 & 1 & $\begin{array}{c}0.01 \% \text { Accuracy P } \\
\text { Transducer }\end{array}$ \\
\hline Rosemount & $3051 \mathrm{C}$ 0-3 PSID & 1 & 2 & $\begin{array}{c}\text { PSID transducer for air } \\
\text { venturis }\end{array}$ \\
\hline Rosemount & $3051 \mathrm{C}$ 0-19 PSIA & 1 & 2 & $\begin{array}{c}\text { PSIA transducer for air } \\
\text { venturis }\end{array}$ \\
\hline Agilent & $33220 \mathrm{~A}$ & 1 & 1 & Waveform Generator \\
\hline Agilent & $34410 \mathrm{~A}$ & 1 & 1 & Digital Multimeter \\
\hline
\end{tabular}

APU inlet mass flow rate is measured by the supplied calibrated bellmouth attached to the 5-vane diffuser section, and the axisymmetric inlet flow will be measured by an 8 " venturi flow meter. Flow rates on both meters will be measured by the two Mensor 6110 high-accuracy pressure transducers. Uncertainty on the bellmouth flow measurement is $0.55 \%$ at $2.0 \mathrm{lbm} / \mathrm{s}$ and $0.65 \%$ at $5.5 \mathrm{lbm} / \mathrm{s}$.

A Precision Filter amplifier system was purchased for the amplification of Kulites and strain gauges and currently has quantity 4 PF 28118 cards installed, capable of amplifying 32 channels and providing up to $20 \mathrm{~V}$ excitation to any sensor type. It interfaces with computer software that allow for the individual customization of all channel settings, including low-pass 4 pole (LP4P) filters from $10 \mathrm{kHz}$ to $100 \mathrm{kHz}$. It has a "self-test" system, 
comprised of an Agilent digital multimeter and waveform generator, which can operate in several different test modes, ranging from a quick "Go/No-Go" test that will check each channel to make sure it is operating properly to a Factory Acceptance Test (FAT) that will adjust each channel for gain, noise, frequency response, etc., and adjust them to factory specifications. A "Go/No Go" test should be run before each data acquisition session, whereas a FAT should be run once per year per manufacturer suggestions.

All data acquisition equipment are located outside of the test cell, with computer monitors for the health monitoring and performance systems, and a large television screen for viewing cameras located in the test cell. A breakout panel for steady pressure channels and thermocouples is also located at the control station and will facilitate reconfiguration of the instrumentation layout for different research setups, as an identical breakout panel will be located inside the test cell. Figure 2.13 shows the electronics rack housing the discussed electronics, as well as the ABB motor control pedestal. Figure 2.14 is a display of the entire Honeywell control station with custom-built 80/20 frame shelving and ample room for future equipment expansion. Figure 2.15 shows the high-speed Precision Amplifier cart, complete with self-test electronics. 
2X Agilent 34921A Multiplexer Modules in 34980A Chassis

1X Agilent 34921A Multiplexer Modules in 34980A Chassis

8X Scanivalve DSA3016 Modules in DSAENCL4000 Chassis

8X Scanivalve DSA3016 Modules in DSAENCL4000 Chassis

PXIE 6224 Card in PXI 1073 Chassis

NI SCB-68 Input Block

ABB Motor Control Pedestal

2X Minuteman Backup Power Supply

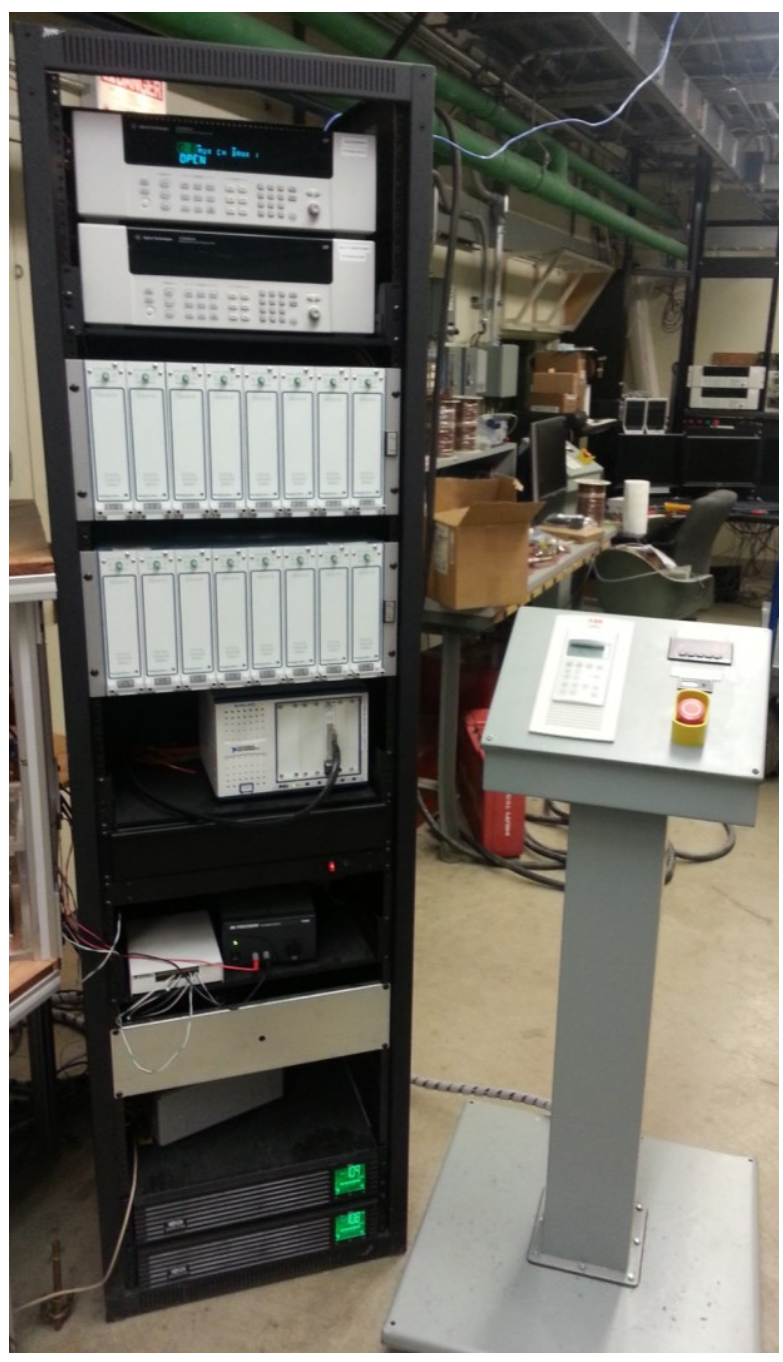

Figure 2.13: Tower with instrumentation and ABB motor control pedestal. 


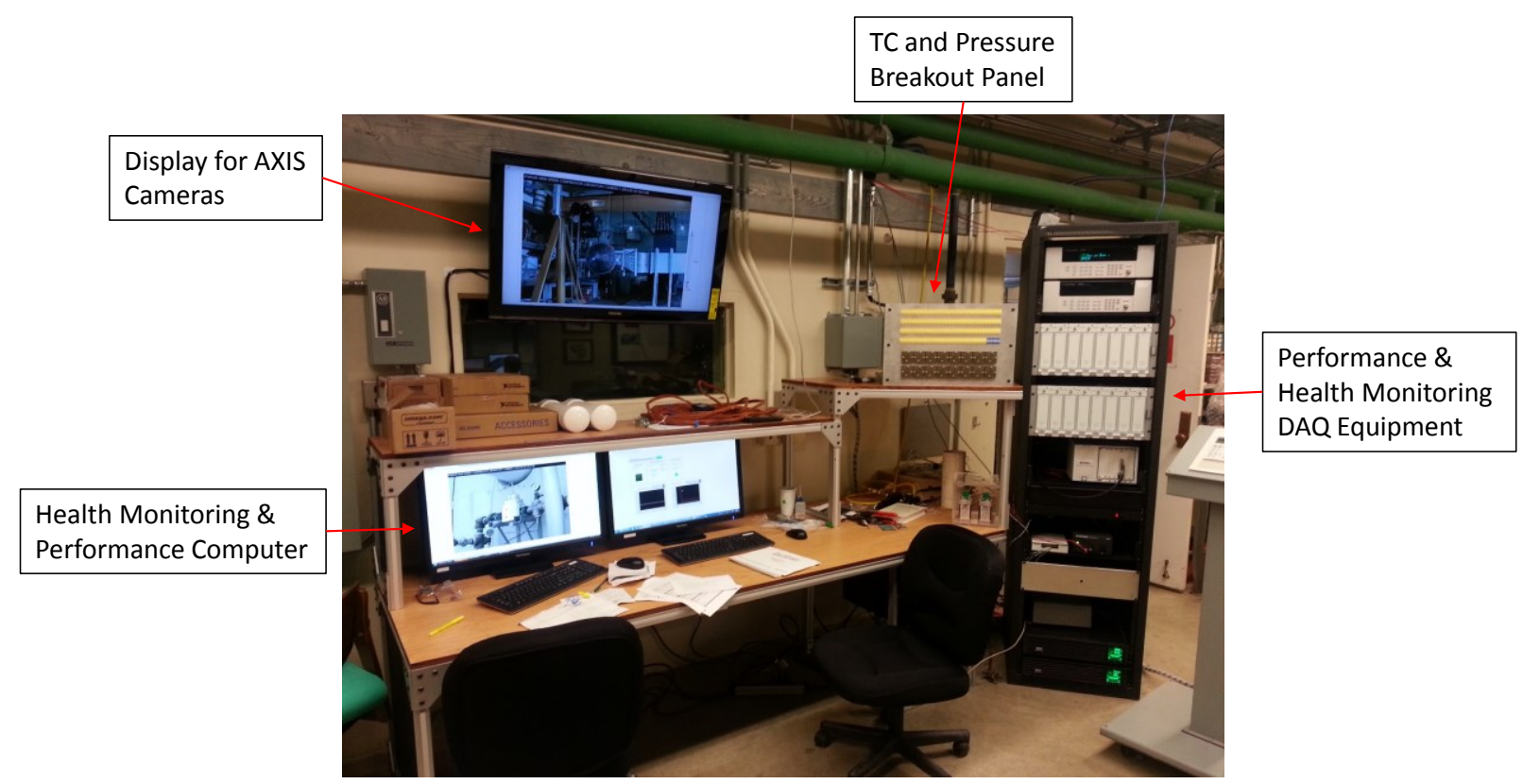

Figure 2.14: View of Honeywell control station.

"Self-Test" System

Agilent 33220A Waveform Generator Agilent 34410A Digital Multimeter

Precision Filter 28000 Chassis with 4X Precision Filter PF 28118 8-Channel Configurable Bridge Amplifiers

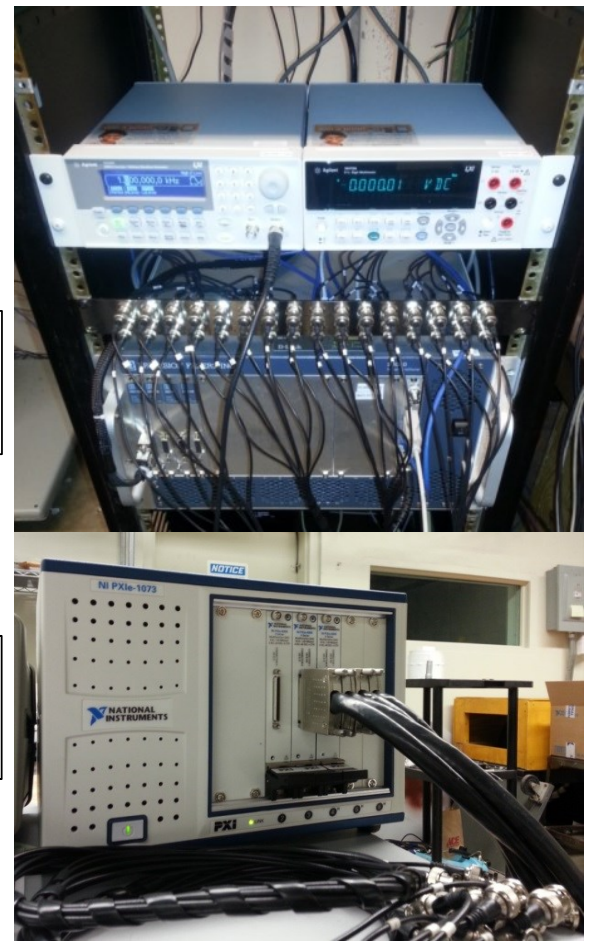

Figure 2.15: High speed data acquisition and amplification hardware. 


\subsection{Oil System Design}

A secondary oil and air system is required for the Honeywell SSCC centrifugal compressor. The oil system will be installed in the test cell and interface with the rig to provide coolant and lubrication to key components in both the compressor and gearbox system. Air lines at varying flow rates and pressures will also be plumbed to the rig to provide purge air to different components of the compressor, as well as provide suction flow for flow path buffer air.

There are many requirements for the oil system for the compressor. The oil must be kept at a temperature between $180^{\circ} \mathrm{F}-220^{\circ} \mathrm{F}$ and total flow rates throughout the system are about 6 GPM. The oil reservoir (specified by Honeywell) must be at least 10 gallons in size, and the oil must be filtered by at least 10 micron filter. Honeywell set up a generalized approach for the oil system, and Purdue completed the detailed design work of getting the system built and functioning. There are three main oil delivery lines that feed the compressor, and each was required to have pressure and flow meters, as well as pressure regulators to set the required pressure. There are five oil return lines connecting the compressor back to the oil tank, with temperature measurements, metal chip detectors, and oil sight glasses on each.

The oil reservoir was designed to be mounted on the wall in the back corner of the test cell, to conserve floor space as well as provide a small gravitational pressure advantage for the oil feel lines. It will house 20 gallons of Mobil Jet II oil, the same oil that will be used in the Cotta gearbox. The same oil is used because there is not a perfect seal at the gearbox output shaft. The height of the oil inside the tank will be measured by a 30 " vertical sight glass installed on the outside and attached to the same vent line coming from the main 
oil tank. A Microsoft webcam will be installed nearby to give the ability to visually monitor the oil level inside the tank while running the compressor. The main line size chosen for the oil system was $1 / 2$ " NPT pipe, as this was large enough to supply the total 6 GPM oil flow rate requirement without significant cost or pressure loss. The outlet of the tank feeds into a Clark Solutions 5 HP gear pump, capable of providing up to 200 psi max outlet pressure, with a VFD so that the pump power output can be adjusted. The higher pressure rating for this pump was chosen so that it would be able to sufficiently provide enough pressure to the oil system so that cheaper, lower flow regulators could be used, and sufficient pressure would be available after all oil system components were installed.

The oil is then fed outside to a heat exchanger enclosure that will house an American Industrial Heat Transfer Inc. heat exchanger. The heat exchanger enclosure was designed to house this heat exchanger as well as the two heat exchangers that will be supplied by Cotta for the gearbox. The heat exchanger for the compressor will be controlled by a thermostat that is connected to a thermocouple on the incoming oil line, so that the fan will run when cooling is needed. Figure 2.16 shows a solid model of the heat exchanger enclosure, constructed from Unistrut and protected from the elements by sheet metal paneling. There are automatic louvers installed in front of each heat exchanger. All heat exchangers exhaust to the center of the enclosure, where hot air is then vented outward by three outward facing louvers. 


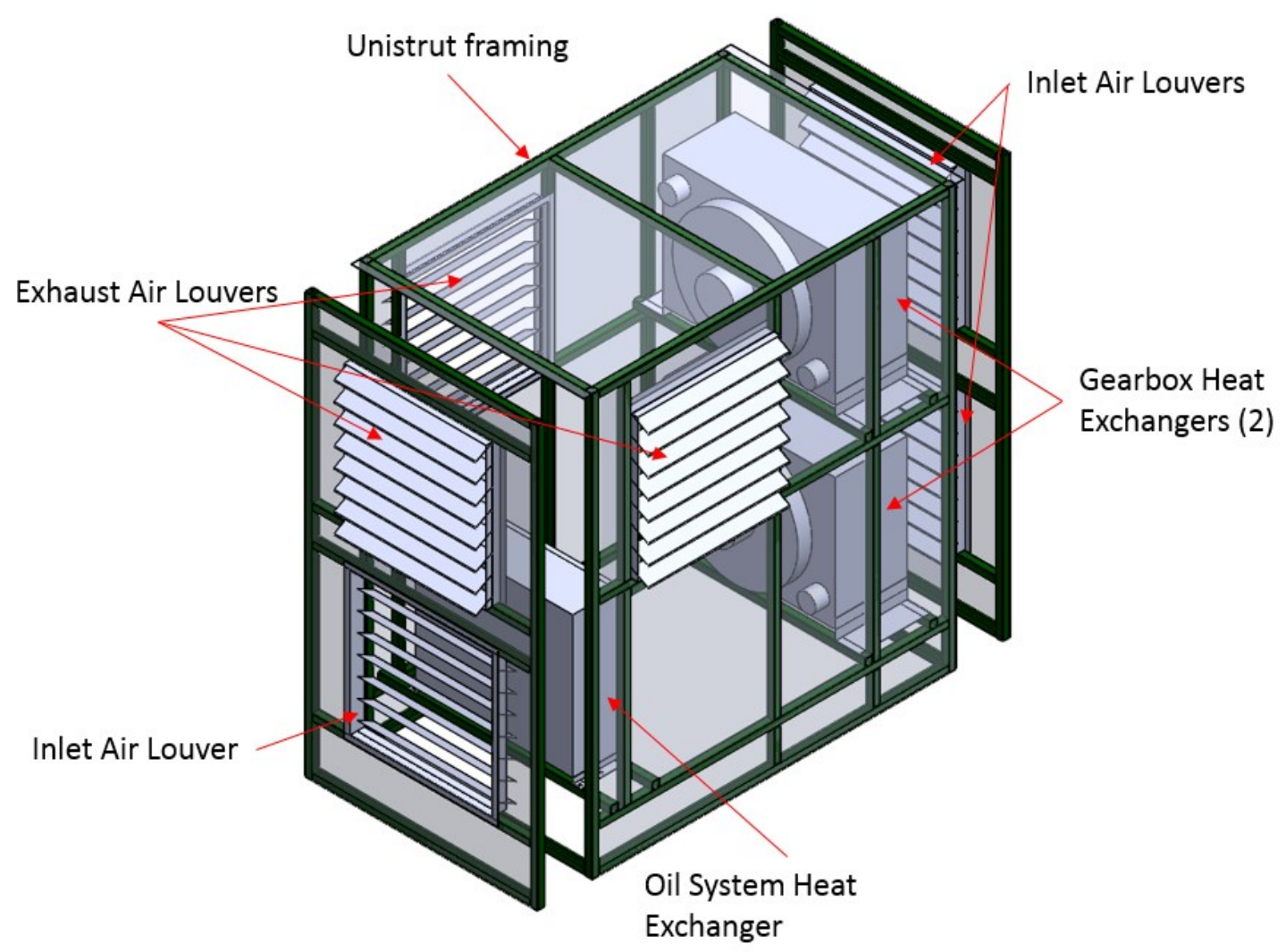

Figure 2.16: 3D SolidWorks model of outdoor heat exchanger enclosure.

The oil coming from the heat exchanger will then be piped back into the test cell and through a 10 micron Norman oil filter. The oil temperature will be recorded with the LabView health monitoring system. The oil will then be fed through a $20 \mathrm{~kW}$ in-line electric heater built by Warren Electric. The electric heater is thermostat controlled, and will need to maintain the oil exit temperature anywhere between $180^{\circ} \mathrm{F}-220^{\circ} \mathrm{F}$, per Honeywell requirements. After leaving the heater, the oil passes through a three-way valve, and this allows the oil system to be switched between different "modes" of operation. One mode of operation will simply have the ball valve return all oil leaving the heat exchanger to the oil tank so that the oil system is in a "heating" mode - this will allow the 20 gallons 
of oil to be brought up from $40^{\circ} \mathrm{F}$ to $220^{\circ} \mathrm{F}$ in as little as 15 minutes, assuming the heater is at full power and there is no heat loss from the oil system. Since the heat loss from the heat capacity of the plumbing hardware, as well as steady state convective and radiative heat losses are difficult to estimate, the power of this heater is oversized. The intention is that the operator of the compressor will have to wait no longer for the oil to come to an appropriate temperature than he/she will have to wait for the variable frequency drive that controls the $\mathrm{ABB}$ motor to come to temperature and be ready to drive the motor.

Once the oil temperature is within an acceptable range, the operator will have to go to the test cell and turn the 3-way valve to the other position, allowing oil to flow freely through the entire compressor. At this point, there will most likely be another drop in the oil temperature as the oil flows through the rest of the cold plumbing and compressor, but hopefully this temperature drop will not add significantly more time to the warm-up procedure.

Once in normal operating mode, the oil will travel from the heat exchanger and then be diverted into three separate lines. These will deliver oil to the Front sump, Aft Sump, and Torque tube of the compressor.

For each of these three sections of the compressor, the oil will be fed through an isolation ball valve, a manual set regulator, a relief valve set at $80 \mathrm{psi}$, an Omega turbine flow meter, another smaller 10 micron Norman filter, and a cross fitted with a pressure gauge and transducer before heading to the compressor. The regulators will be manually set for the pressure ranges required for each compressor component and monitored visually by gauges as well as electronically through LabView. In case of a regulator failure, the exit lines from the relief vales are routed back to the main oil holding tank. The flow meters are 
constructed from stainless steel and are capable of handling the high oil temperatures. The extra filters were put in place to capture any remaining particles in the oil system generated from plumbing, the heat exchanger, or in case the regulators or flow meters fail and send debris into the oil line.

There are 5 separate lines returning oil from the compressor, with an expected temperature of $205^{\circ} \mathrm{F}-225^{\circ} \mathrm{F}$, assuming a $180^{\circ} \mathrm{F}-200^{\circ} \mathrm{F}$ inlet oil temperature. The oil return lines are fitted each with oil sight glasses, thermocouples, chip detectors, scavenge gear pumps, and check valves before being manifolded together and dumped back into the oil tank. The thermocouples and oil sight glasses will monitor the flow of oil (the sight glasses will be viewed with another remote camera), and the temperature of each oil line will be measured and recorded with LabView. The chip detectors are fitted to the lines to detect chips coming from excessive wear within the compressor. The scavenge pumps will pump the oil back to the tank through check valves to prevent the backflow of oil from one line to a lower pressure line.

In addition to the main oil lines, the Front Sump and Aft sump will have oil vapor return lines that will be routed to the main oil tank. The Aerodyne slip ring will have its own oil mister cart installed for lubrication and cooling, and the oil lines supplied to the mister cart are a completely self-contained system. Lastly, the oil tank will be vented to ambient through an air/oil separator to equalize pressure both inside and outside of the tank.

The oil circuit for the Cotta gearbox consists of the following main components: an oil pump, the gearbox, and two heat exchangers plumbed in line with respect to each other. The oil level within the Cotta gearbox will be monitored via an installed oil sight glass on the gearbox housing, and this level will be watched to see if significant oil transfer between 
the gearbox and the compressor is occurring over time. Oil will be manually transferred to the respective oil reservoir in either the gearbox or compressor oil system if necessary. Table 2-4 shows the required pressure, temperature, and flow rate for the three inlet oil lines to the compressor.

Table 2-4: Honeywell oil requirements for sections of SSCC rig.

\begin{tabular}{|c|c|c|c|}
\hline Component & Pressure Required & Temp Required & Flow Rate \\
\hline Front Sump & $60-70 \mathrm{psi}$ & $180 \mathrm{~F}-220 \mathrm{~F}$ & $1.6-2.0 \mathrm{GPM}$ \\
\hline Aft Sump & $60-70 \mathrm{psi}$ & $180 \mathrm{~F}-220 \mathrm{~F}$ & $1.57-1.9 \mathrm{GPM}$ \\
\hline Torque Tube & $40-50 \mathrm{psi}$ & $180 \mathrm{~F}-220 \mathrm{~F}$ & $1.53-2.0 \mathrm{GPM}$ \\
\hline
\end{tabular}

The legend for the oil system is shown in Figure 2.17, and the oil system schematic is shown in Figure 2.18. 


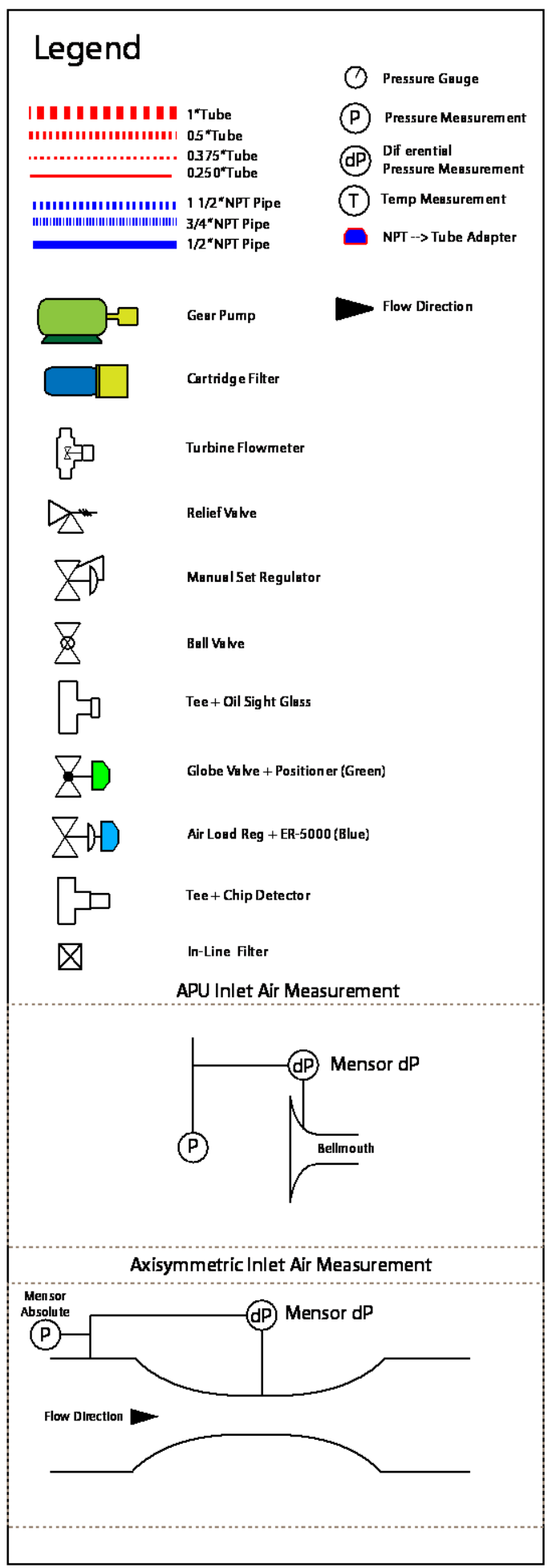

Figure 2.17: Oil and air system legend. 


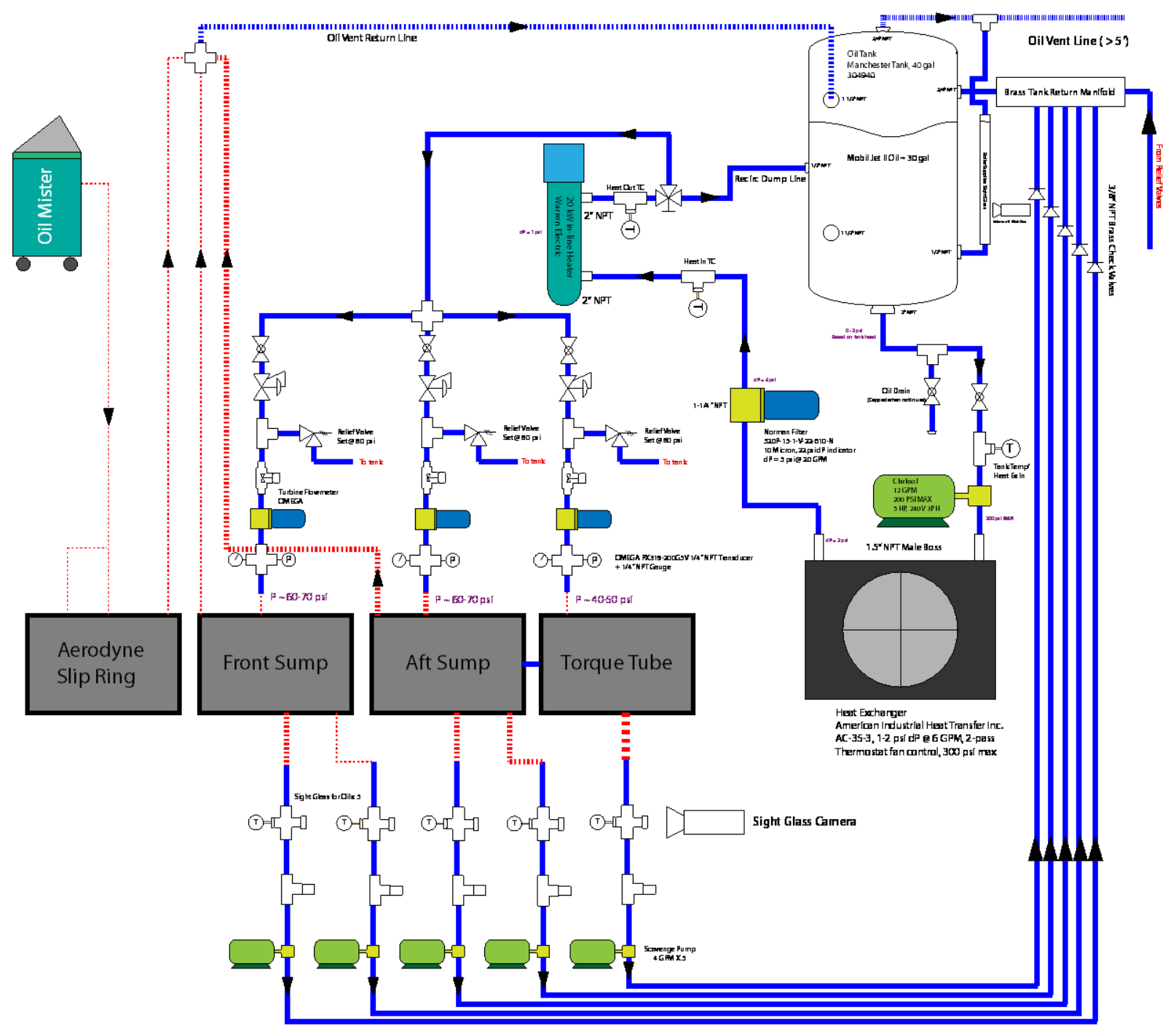

Figure 2.18: Honeywell oil system.

\section{$2.6 \quad$ Air System Design}

The air system is divided in to three separate regions of the compressor: Forward, Mid and Aft sections. The forward section requires plumbing to two main circuits, one providing a negative gauge pressure suction flow, and one providing positive pressure for the Forward Carbon Seal Buffer Air. The Mid section requires no flow, but the aft section requires two different air pressures and flow rates to control the thrust piston. The Mid 
section will need backpressure adjustment and flow metering for the Diffuser Match Bleed. The Aft section will need two separate metered and backpressure controlled air lines for Back Face Bleed and Thrust Piston Vent/Aft Carbon Seal Buffer Air Out. All three of these outlet lines will be vented to ambient pressure. All air lines going to or coming from the compressor will need to be electronically regulated, which requires the automation and control schemes that will be implemented through a health monitoring code written in LabView.

The air supplied to the compressor will come from the air system already plumbed for the air ejector used in the APU inlet testing. Once the compressor is installed, the air ejector will no longer be in use, and thus, the air circuit used to provide the motive air for that system will be diverted into the test cell and deliver all the required air for the secondary flow systems. The large $\mathrm{Cv}=12$ regulator will have more than enough flow capability to provide air at the required pressure and flow rate for the whole compressor system. The source of the air will come from the Zucrow high pressure air system, with pressure varying from $600 \mathrm{psi}$ to $2,200 \mathrm{psi}$, depending on the amount of air stored in the outdoor tanks at any one time. The compressor will be able to run whether or not the tanks are at either pressure, since both pressures will still provide plenty of air flow. The air flow will be filtered by the Norman cartridge air filter to 20 micron.

The air from this large TESCOM regulator will be piped into the test cell in $1 \frac{1 / 2}{2}$ NPT pipe, and diverted to four different regulators, each delivering a different pressure. All of the regulators will be the same TESCOM DK series regulator, and each will be controlled by an ER-5000 electronic air regulator that will receive its control air pressure from the shop air line, which can vary from 80-110 psi. The flow rate for the ER-5000 
control will be negligible. Each of the ER-5000 units will be connected to the health monitoring computer for control.

For the flow path Buffer Air, a vacuum is required, as the air pressure for this line needs to be between 10.97 and 13.05 psia. A custom designed ejector supplied by Fox Valve will provide this vacuum, which will require $30 \mathrm{psi}$ at $0.017 \mathrm{lbm} / \mathrm{s}$ to create the maximum required suction flow. The company did not provide off-design flow curves for the ejector, but based on experience with the large APU-style Schutte and Koerting ejector, it is assumed that the motive air pressure and flow rate for the Foxvalve ejector will be able to adequately provide suction throughout the entire required flow range. Forward carbon seal buffer air, thrust piston buffer air, and thrust piston supply air will simply be plumbed with a DK Series Regulator, ER-5000, relief valve, and downstream pressure transducer. Air relief valves will be connected to the outlet line coming from each of the DK series regulators and will be sized to $125 \%$ maximum required delivery pressure.

The Diffuser Match Bleed, Back Face Bleed, and Thrust Piston Vent will all experience elevated temperatures from $170^{\circ} \mathrm{F}$ to $550^{\circ} \mathrm{F}$. TESCOM was unable to supply backpressure regulators that will be able to either provide a high enough temperature or flow capability. Instead, these lines will be fitted with globe valves controlled by a mechanical positioner that will be controlled via LabView to provide the required backpressure for each operating condition. The Diffuser Match Bleed and Back Face Bleed will be fitted with ASME standard venturis and Rosemount absolute and differential pressure transducers to monitor the exit flow rates. Rosemount absolute pressure transducers with a range of 0-30 psia were chosen but calibrated from 0-19 psia, and the differential pressure transducers have a range of 0-3 psid. Table 2-5 shows the flow rate 
and conditions used for venturis sizing. Pressure drop calculations were performed by the venturi manufacturer, Flowmaxx.

Table 2-5: Venturi design for Diffuser Match Bleed (1), Diffuser Back Face Bleed (2).

\begin{tabular}{|c|c|c|c|c|}
\hline Venturi No. & Flowrate [lbm/s] & dP [psi] & Temp [F] & Condition \\
\hline 1 & 0.0448 & 1.49 & 370 & $100 \%$ Speed, Hot \\
\hline 1 & 0.0560 & 2.60 & 370 & $125 \%$ Max Flow Rate \\
\hline 1 & 0.0448 & 1.10 & 170 & $100 \%$ Speed, Cold \\
\hline 1 & 0.0234 & 0.37 & 370 & $60 \%$ Speed, Hot \\
\hline 1 & 0.0234 & 0.28 & 170 & $60 \%$ Speed, Cold \\
\hline 2 & 0.128 & 1.59 & 550 & $100 \%$ Speed, Hot \\
\hline 2 & 0.160 & 2.77 & 550 & $125 \%$ Max Flow Rate \\
\hline 2 & 0.128 & 1.07 & 239 & $100 \%$ Speed, Cold \\
\hline 2 & 0.0615 & 0.34 & 550 & $60 \%$ Speed, Hot \\
\hline
\end{tabular}

To size the regulators for the air system, the $C_{v}$ of each valve was calculated. For each regulator, Honeywell supplied required pressures and flow rates for at $60 \%$ and $100 \%$ speed operation points. The required $C_{v}$ for each valve was calculated at each operating condition, and the larger $C_{v}$ value was used to size the valve. Eqn. 2-1 and Eqn. 2-2 were used for $C_{v}$ calculations. The result of the calculations is shown in Table 2-6. Figure 2.17 shows the air and oil system legend, and Figure 2.19 is the schematic for the air system. Construction of the oil and air system will be completed in the summer of 2014. 


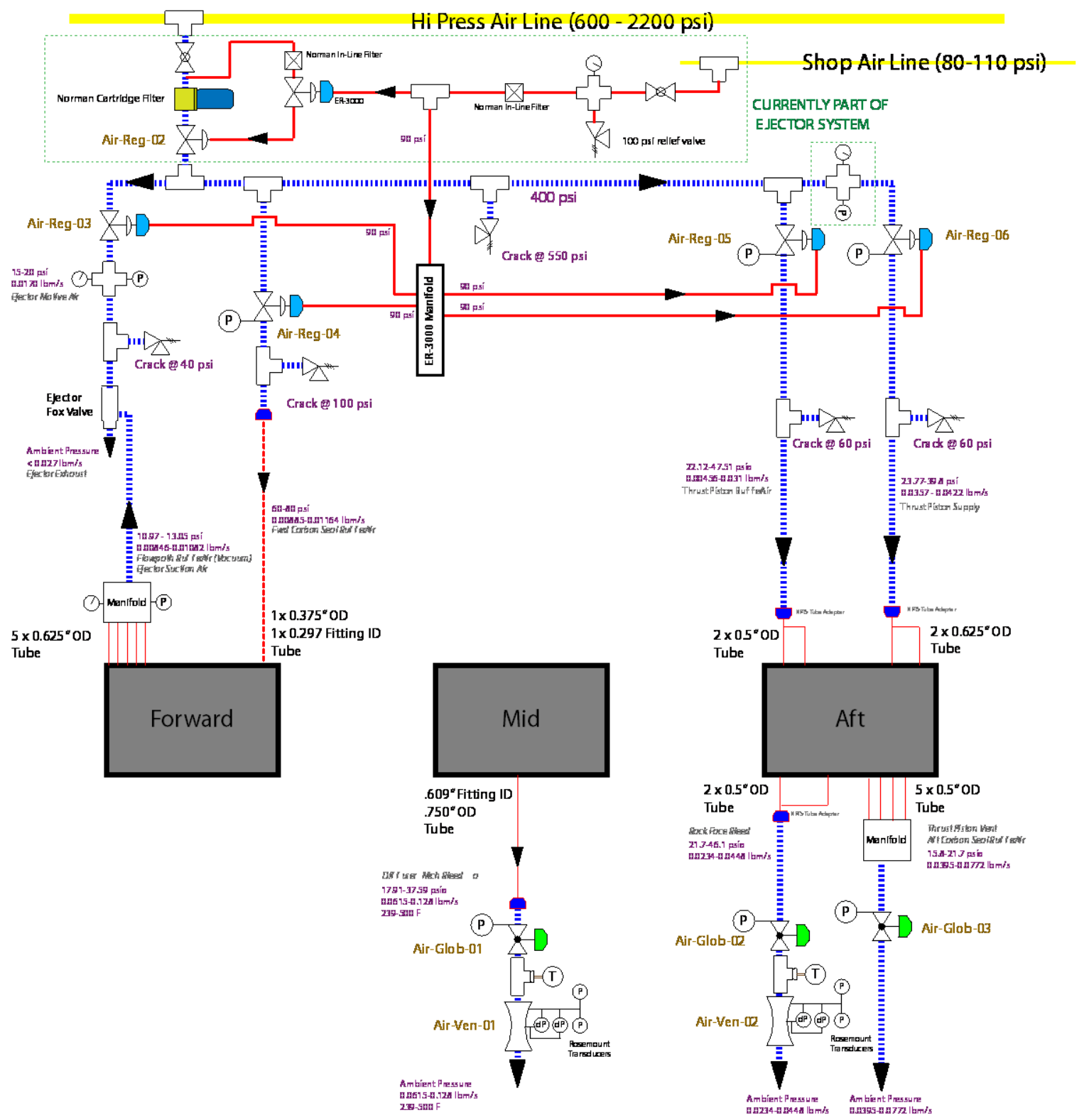

Figure 2.19: Honeywell air system. 
Table 2-6: Air flow requirements for all air regulators in Honeywell air system.

\begin{tabular}{|c|c|c|c|c|c|c|c|c|c|c|c|}
\hline & \multirow{2}{*}{$\frac{\mathbf{P 1}}{[\mathrm{psia}]}$} & \multirow{2}{*}{$\frac{\text { P2 }}{[\mathrm{psia}]}$} & \multirow{2}{*}{$\begin{array}{c}\text { Flowrate } \\
{[\mathrm{lbm} / \mathrm{s}]}\end{array}$} & \multirow{2}{*}{$\frac{\mathbf{T}}{[\mathrm{F}]}$} & \multirow[t]{2}{*}{ Cv Req. } & \multirow{2}{*}{$\frac{\mathbf{P 1}}{[\mathrm{psia}]}$} & \multirow{2}{*}{$\frac{\text { P2 }}{[\text { psia }]}$} & \multirow{2}{*}{$\begin{array}{c}\text { Flowrate } \\
{[\mathrm{lbm} / \mathrm{s}]} \\
\end{array}$} & \multirow{2}{*}{$\begin{array}{c}\mathbf{T} \\
{[\mathrm{F}]} \\
\end{array}$} & \multirow[t]{2}{*}{ Cv Req. } \\
\hline & & & & & & & & & & & \\
\hline APU Ejector Reg & Air-Reg-02 & 2200 & 400 & 0.05591 & 0 & 0.040 & 600 & 400 & 0.09844 & 110 & 0.287 \\
\hline Ejector Motive Flow & Air-Reg-03 & 400 & 15 & 0.00680 & 70 & 0.029 & 400 & 30 & 0.01360 & 70 & $\mathbf{0 . 0 5 7}$ \\
\hline Fwd Carbon Seal Buffer Air & Air-Reg-04 & 400 & 60 & 0.00885 & 70 & 0.037 & 400 & 80 & 0.01164 & 70 & 0.049 \\
\hline Thrust Piston Buffer Air & Air-Reg-05 & 400 & 22.14 & 0.00456 & 70 & 0.019 & 400 & 48 & 0.03100 & 70 & 0.131 \\
\hline Thrust Piston Supply Air & Air-Reg-06 & 400 & 24 & 0.03570 & 70 & & 400 & 40 & & 70 & 0.178 \\
\hline Diffuser Match Bleed & Air-Glb-01 & 17.91 & 14.7 & 0.06150 & 239 & 8.405 & 37.59 & 14.7 & 0.12800 & 550 & 7.930 \\
\hline Back Face Bleed & Air-Glb-02 & 21.7 & 14.7 & 0.02340 & 170 & 2.095 & 46.1 & 14.7 & 0.04480 & 370 & 2.051 \\
\hline Thrust Piston Vent & Air-Glb-03 & 15.8 & 14.7 & 0.06150 & 80 & 12.405 & 21.7 & 14.7 & 0.03950 & 160 & 3.508 \\
\hline
\end{tabular}




\section{CHAPTER 3. SETUP FOR AXIAL COMPRESSOR EXPERIMENTS}

\subsection{Motivation and Requirements}

This chapter describes the instrumentation used to measure the 44 EO excitation of the $1 \mathrm{~T}$ vibratory mode of Rotor 2 in the Purdue 3 stage axial compressor. The upstream and downstream vane counts are 44 , and this results in a resonant speed around $74 \%$ speed, or 3700 RPM. The aim of this project was to create a detailed high quality data set by which predictive tools could be validated. Characterizing boundary conditions needed for CFD was just as important as characterizing the response and unsteady forcing function using fast response instrumentation. The data acquired during this study can be immensely useful to aid in the development of advanced vibrational response predictive tools, where experimental verification of predictions are sparse. It will allow for the tuning and calibration of computer analyses, as well as provide insight into advanced data processing techniques aimed at quantifying the unsteady flow field associated with bladed disks in resonance. The Campbell diagram for this compressor, Figure 3.1 shows that the other crossings associated with the $44 \mathrm{EO}$ excitation are outside of the operating range. 


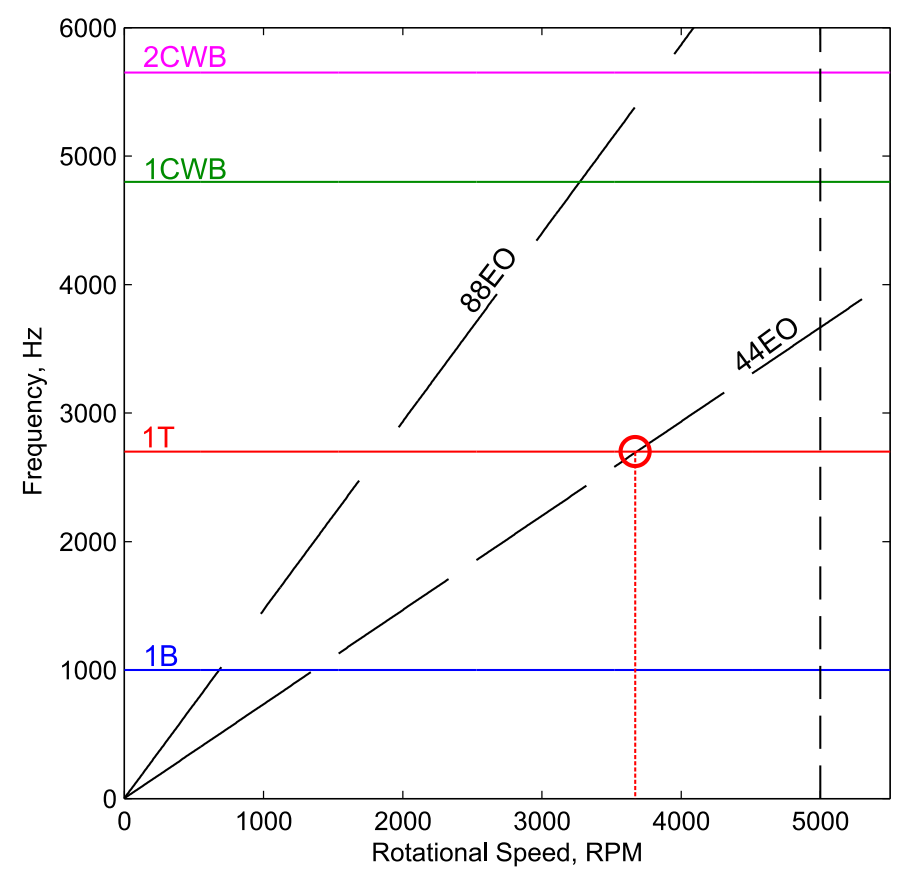

Figure 3.1: Campbell diagram showing R2 1T, where GUIde IV is centered around.

To measure the vibratory response of R2, an optical Non-Intrusive Measurement System (NSMS) was used to track the tip deflection of each individual blade as the rotor went through resonance. Fast-response Kulite pressure transducers were mounted both over Rotor 2 and downstream, in Stator 2, to measure the unsteady pressure field. Hotwire measurements were used to characterize the unsteady velocity both upstream and downstream of R2. Data are acquired at two loading conditions, focusing on $50 \%$ and $80 \%$ span locations. All fast response data were taken at a constant speed near resonance and also while slowly sweeping (accelerating or decelerating) through resonance speeds.

Vibrometry data of all stator sections was acquired to quantify the response spectra of the non-rotating hardware in the compressor as well as to aid in the selection of safe steady compressor operating speeds. A laser scan of all three rotor blisks was performed 
by Aerodyne, and this data can be used to help identifying the mistuning pattern unique to each blisk assembly in addition to documenting as-built rotor geometry.

\subsection{P3S Research Facility}

Experiments performed in the Purdue 3-stage (P3S) axial compressor was used to acquire all data pertaining to aeromechanics studies. The compressor driveline consists of an $\mathrm{ABB}$ 1,400 HP motor that drives a 5:1 gearbox. The motor is controlled by a Variable Frequency Drive (VFD) that is manually operated outside of the test cell and is capable of holding the motor at set speed within $0.01 \%$. Programmable ramp rates for compressor speed were used to acquire unsteady data through rotor resonances.

Incoming air is passed through a settling chamber with filters and aluminum honeycomb that creates uniform flow, and it is then passed through an ASME standard long form venturi to measure massflow. The air is then diverted by a nosecone to the flow annulus (24" in diameter with a 5/6 hub-to-tip ratio) at which point it passes through the IGV and through the rest of the blading. A movable throttle valve is used to set the loading for the compressor, and the flow is exhausted out the side of the building. Figure 3.2 shows the layout of the test cell including the inlet duct system outside. 


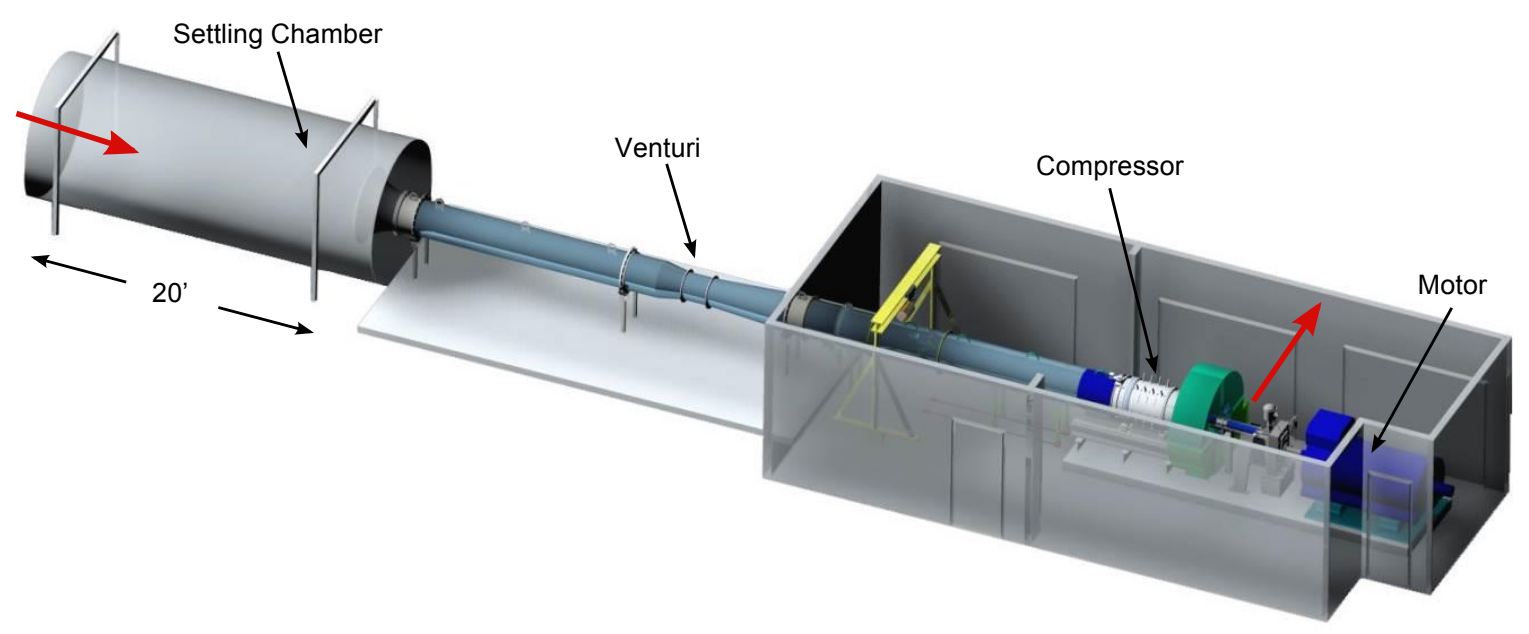

Figure 3.2: Purdue 3 stage axial compressor research facility.

The gearbox output shaft is connected to the axial compressor, which consists of an Inlet Guide Vane (IGV) and three stages. Compressor speed is verified using an optical tachometer on the gearbox output shaft and this speed is used to manually maintain the compressor within a desired corrected speed within $0.1 \%$. At each station, Figure 3.3, there exist 7-element rakes for measuring stagnation pressure and temperature in addition to static taps for casing pressure. Also, at each station there exists mounting ports for traversing mechanisms for probes such as hotwire and kiel head total pressure probes used for this research. Overall uncertainty in temperature measurements is 0.3 degrees Celsius. The numbering scheme of each station is shown in Figure 3.3. Mass flow rate is calculated from measurements by very high accuracy differential pressure transducers connected to an ASME standard long-form venturi, with a maximum relative uncertainty in mass flow rate of $1.4 \%$ when taking into account ambient density and relative humidity. Ambient pressure is measured with a high accuracy barometric pressure transducer with a full scale uncertainty of $+/-0.073 \%$. 


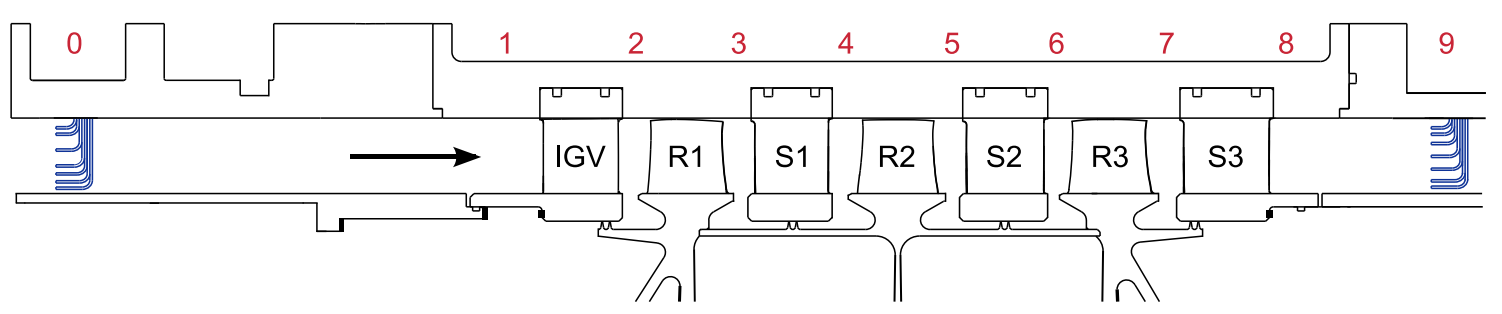

Figure 3.3: Cross section of Purdue 3-stage axial compressor, showing relevant station numbers.

Each vane row is connected to actuators that move the vanes in the circumferential direction (up to 2 vane passages) allowing stationary probes to acquire data in the pitchwise direction. The IGV, Stator 1 (S1), and Stator 2 (S2) consist of 44 vanes, whereas Stator 3 (S3) has 50 vanes. Rotor 1 (R1), Rotor 2 (R2), and Rotor 3 (R3), have 36, 33, and 30 blades, respectively. This project focuses primarily on S1, R2, and S2, as the aeromechanic response of R2 is greatly affected by its neighboring stators.

\subsection{NSMS Measurements}

An Agilis NSMS system was used to measure Rotor 2 blade response as it passed through 1T resonance condition. The system consisted of 8 fiber-based optical probes, laser and detector boxes, and an NI 5112 ADC data acquisition chassis. The laser module generates a signal that is sent to each of the 8 probes that shines on each blade as it passes by the sensor. Each probe is also equipped with a sensing optical cable that transmits the reflection of the laser off of the tip of the blades as they pass by. The timing of each blade arrival and the correlation of arrival times with all 8 sensors circumferentially placed around the compressor is performed by the Agilis NSMS software to detect the amplitude 
and phase of the vibration of each individual blade as a function of time. One measurement of amplitude and phase is made per blade per rotation of the compressor, and thus, the amount of data that the NSMS system acquires is a function of the ramp rate of the compressor rotational speed. The probes were placed at the trailing edge of R2 and arranged circumferentially so that the error in the 44 EO driven response was minimized. Figure 3.4 shows the angular placement of all 8 probes in the casing.

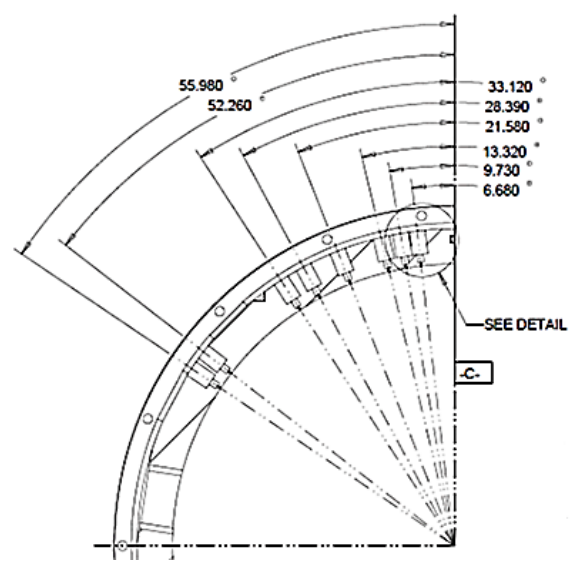

Figure 3.4: NSMS Probe circumferential placement in casing, forward facing aft.

The ABB motor that drives the compressor was programmed to operate the compressor at speeds corresponding to R2 resonance at sweep rates varying from 2.11 $\mathrm{RPM} / \mathrm{s}$ to $5 \mathrm{RPM} / \mathrm{s}$. Traditionally, the critical sweep rate, as calculated by the method of Von Flotow (2003), for this compressor and this Campbell diagram crossing is $15 \mathrm{RPM} / \mathrm{s}$, but lower sweep rates were used to achieve a higher time-domain resolution in flow field data. The sweep rate of $2.11 \mathrm{RPM} / \mathrm{s}$ was the lowest achievable by the ABB motor controller. 


\subsection{Stator Loading Measurements}

Since the budget and scope of this project did not allow for rotor-mounted instrumentation, unsteady surface pressures were acquired on the downstream vane to provide unsteady loading data for code validation processes. To provide a removable S2 passage that could be sent to Kulite for sensor installation, a new S2 half ring was designed that split into three sections. The part was constructed of 17-4 PH Stainless Steel and EDM cut into segments. The blades of the removable passage were machined to accommodate a total of 16 Kulite LQ-062 pressure transducers, 8 on each blade. They were positioned at $50 \%$ span and $80 \%$ span, at $10 \%, 20 \%, 30 \%$, and $40 \%$ chord on the pressure side of one blade and the suction side of another. The Kulites had no screen resulting in a higher frequency response, as much as $100-150 \mathrm{kHz}$ per manufacturer's specifications. Figure 3.5 shows the stator section cutout without installed Kulites, and Figure 3.6 shows the position from the Stator 2 half-ring from which the 2-vane section was EDM cut. Figure 3.7 is a profile illustration of the Kulite placement within the thickness of the vane. This cross section is representative of both span wise locations for suction side sensors. The pressure side sensors are similarly installed but on the opposite side of the vane. 


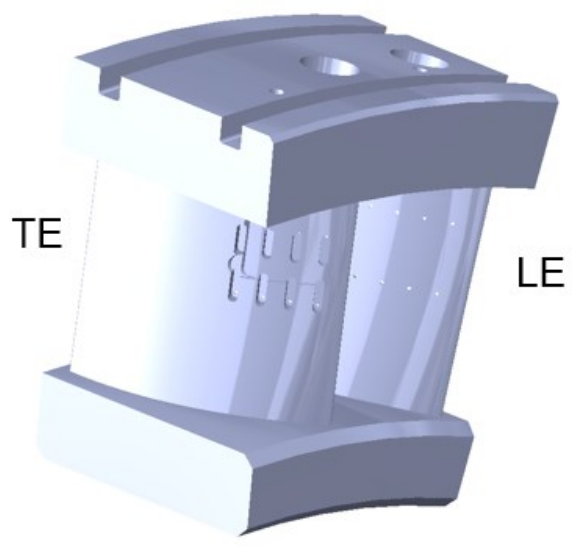

Figure 3.5: 3D model of stator before Kulite installation.
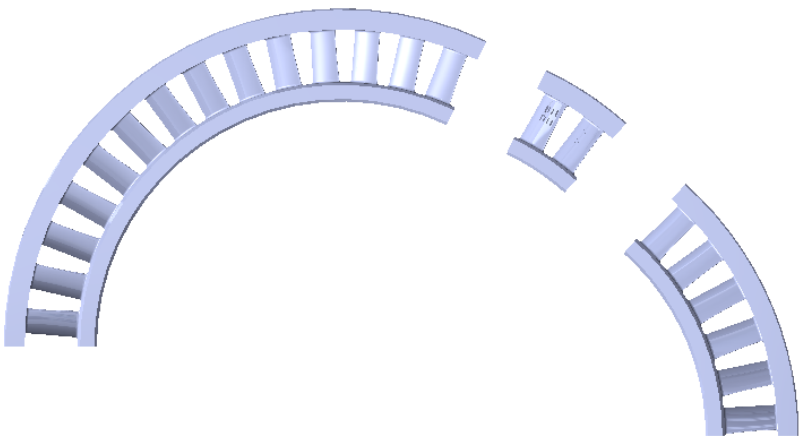

Figure 3.6: Stator 2 EDM cut into three sections for stator Kulite instrumentation.

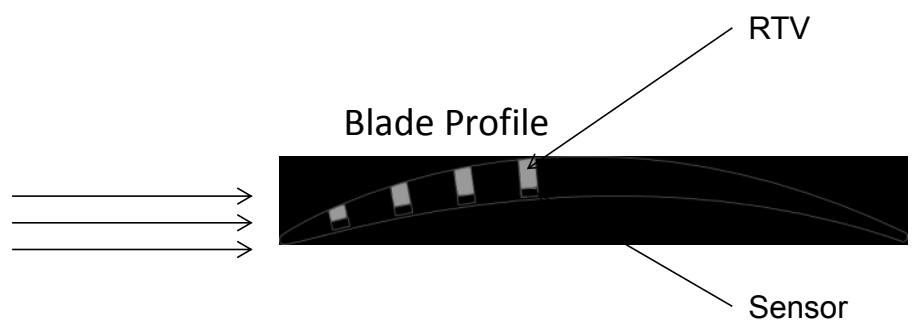

Figure 3.7: Depiction of stator blade with Kulite sensors installed at 10\%, 20\%, 30\% and $40 \%$ chord. 


\subsubsection{Stator Vibrometry Testing}

The frequency of vibration of the new S2 ring could be affected by this modification, and this frequency had to be determined before being used in the 3-stage compressor. An uncertainty of $+/-20 \%$ of the vibration frequency is commonly associated with shrouded stators because of the difficulty of modeling the proper boundary conditions. Thus, the frequencies must be determined experimentally to reduce this uncertainty. Vibrometry testing was conducted at Wright Patterson Air Force Research Laboratory by means of a piezo-electric transducer and an optical laser displacement measurement system. One halfring of each stator section was tested, as well as the IGV, and the three-part Stator 2 half ring that has been sectioned for Kulite transducer installation. In total, 7 individual stator sections were individually tested for frequency response within the frequency band of the compressor operating range.

Initially, the stator sections were placed in the compressor casing, and the casing itself was excited by a piezo transducer, which was fastened to the compressor casing and used a large internal neodymium magnet to shake the casing, and acoustic waves from a loudspeaker. Due to the large mass of the casing half and stator sections, the system was extremely damped, and it was determined that no unsafe response would occur if the vanes were properly mounted into the casing. The stator sections were also removed from the casing and individually excited by physically mounting a piezo-electric transducer to threaded bosses already present on the stator shroud to obtain some frequency information for future knowledge.

Figure 3.8 shows the setup for the vibrometry testing with actuator attached directly to the half ring that was removed from the casing. 


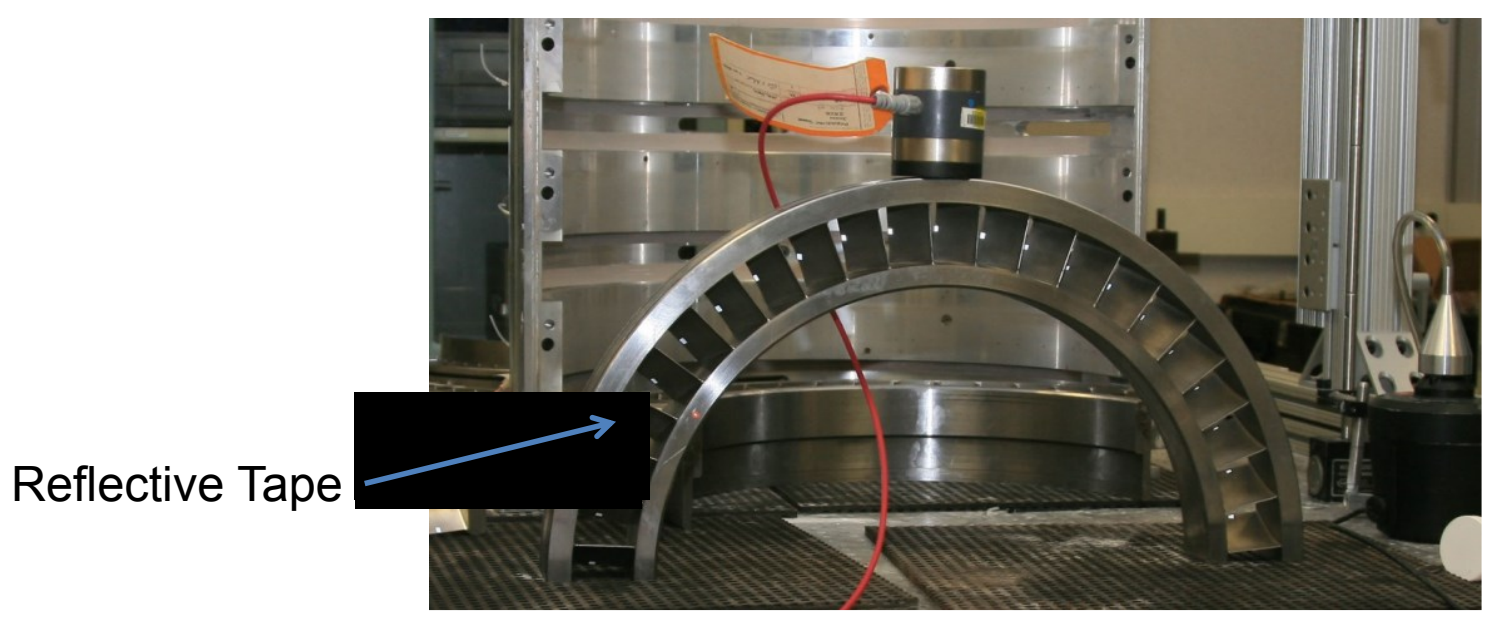

Figure 3.8: Vibrometry testing of stator half ring with piezo-electric driver at WPAFB.

The shiny spots on each vane are the reflective tape used to get a good signal from the vibrometer. The new 3-piece S2 half ring was also tested. Figure 3.9 shows the setup for the removable passage. This was done prior to the Kulite installation to avoid damaging the sensors.

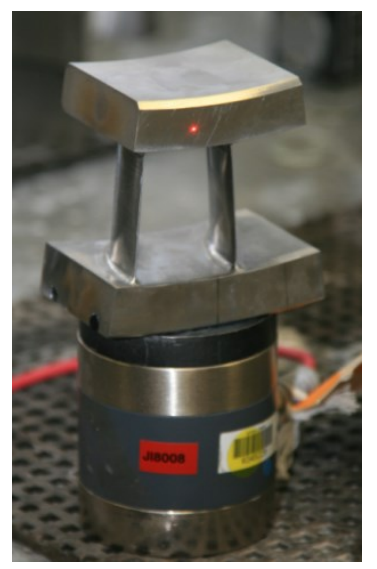

Figure 3.9: Vibrometry testing of stator 2 insert. Only case where piezo driver supported the stator section being tested.

Each blade was marked with reflective silver tape near the leading edge, and the stators were placed upright on a vibration isolating rubber matt. The piezo transducer 
excited each stator section with a frequency band ranging from $0.2-5 \mathrm{kHz}$, repeating this excitation for each individual blade as blade displacement was recorded during the sweep by focusing the laser on each blade's silver tape. To get blade mode shapes throughout the excitation range, one blade from each of the 7 tested sections was completely covered in reflective tape, and the laser measured individual displacements at over 100 points over the surface of the blades. Because this modal analysis was labor intensive, one blade from each section was chosen to be representative of the modal shapes of the other blades on each stator section. The FT response plots shown are an overlay of the FT of each individual blade as excited by the piezo-electric transducer and represent the full frequency response envelope of each stator section.

Referring back to Figure 3.6, the EDM cut stator half-ring can be divided into three sections_-"large", "medium" and "small". Table 3-1 shows the results of all 7 tested stator sections, documenting the mean frequencies at which the vibration amplitude was greater than or equal to.005 mils.

Table 3-1: Frequencies of peaks above .005 mil during testing.

\begin{tabular}{|r|c|c|c|c|c|c|c|}
\hline & IGV & S1 & S2 Large & S2 Med & S2 Small & S2 Full & S3 \\
\hline & {$[\mathbf{H z}]$} & {$[\mathbf{H z}]$} & {$[\mathbf{H z}]$} & {$[\mathbf{H z}]$} & {$[\mathbf{H z}]$} & {$[\mathbf{H z}]$} & {$[\mathbf{H z}]$} \\
\hline 1 & 1352 & 1606 & 685.2 & 1811 & 3185 & 2295 & 1583 \\
\hline 2 & 2077 & 2234 & 1204 & 4075 & 3826 & 2678 & 1692 \\
\hline 3 & 3009 & 2573 & 1831 & 4948 & & 2831 & 2738 \\
\hline 4 & 3102 & 2708 & 2319 & & & 3656 & 2873 \\
\hline 5 & 3784 & 2809 & 2573 & & & 4149 & 3363 \\
\hline 6 & 3973 & 3612 & 3384 & & & 4244 & 4231 \\
\hline 7 & 4494 & 4045 & 3788 & & & 4356 & 4447 \\
\hline 8 & 4601 & 4173 & 3956 & & & & 4621 \\
\hline 9 & & 4269 & 4382 & & & & 4916 \\
\hline 10 & & & 4652 & & & & \\
\hline
\end{tabular}




\subsubsection{Stator Kulite Pressure Measurements}

After the vibrometry testing was complete, the removable passage was shipped to Kulite for sensor installation. When the part arrived at Purdue, it was immediately noted that one of the sensors was not working - the $50 \%$ span pressure side sensor at $20 \%$ chord. Figure 3.10 shows the passage with the sensors installed, and Figure 3.11 shows a view of the RTV on the back side of the instrumented vanes.

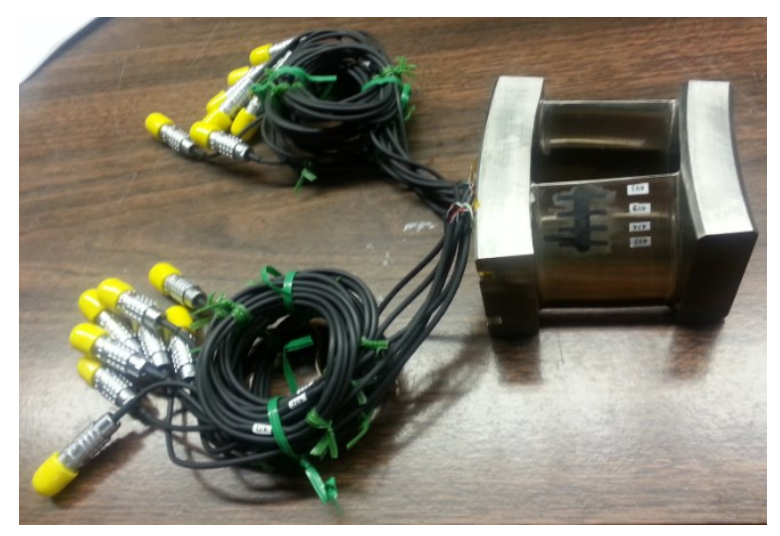

Figure 3.10: Stator section upon arrival from Kulite.

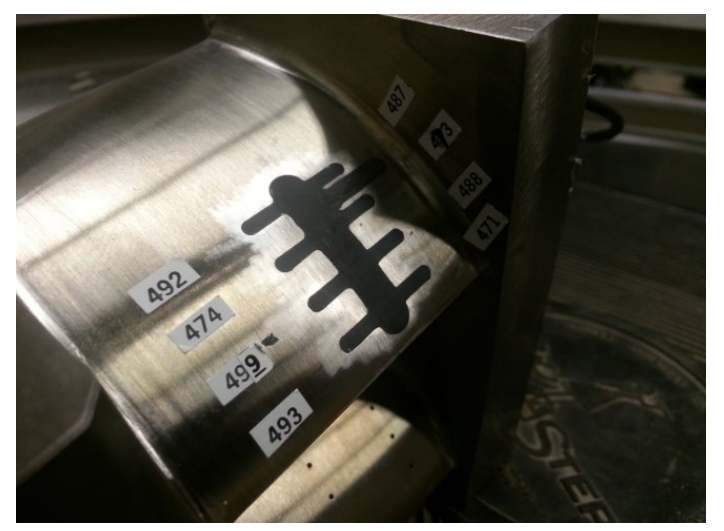

Figure 3.11: Close up of stator section showing RTV on backside of installed Kulites.

The stator section Kulites were calibrated for the mean operating pressure range. A calibration chamber was constructed from 6" cast aluminum pipe that was 6" long with 
caps on each end. The stator was installed in the chamber by a short piece of threaded rod that was threaded into one of the machined and tapped threads in the shroud of the stator, shown in Figure 3.12. The LEMO connectors were fed out of two holes drilled in one of the caps and sealed off with silicone sealant. Heat shrink used on the outside of each of the wires was also sealed at each end with silicone to keep air from leaking through the heat shrink and out of the calibration chamber.

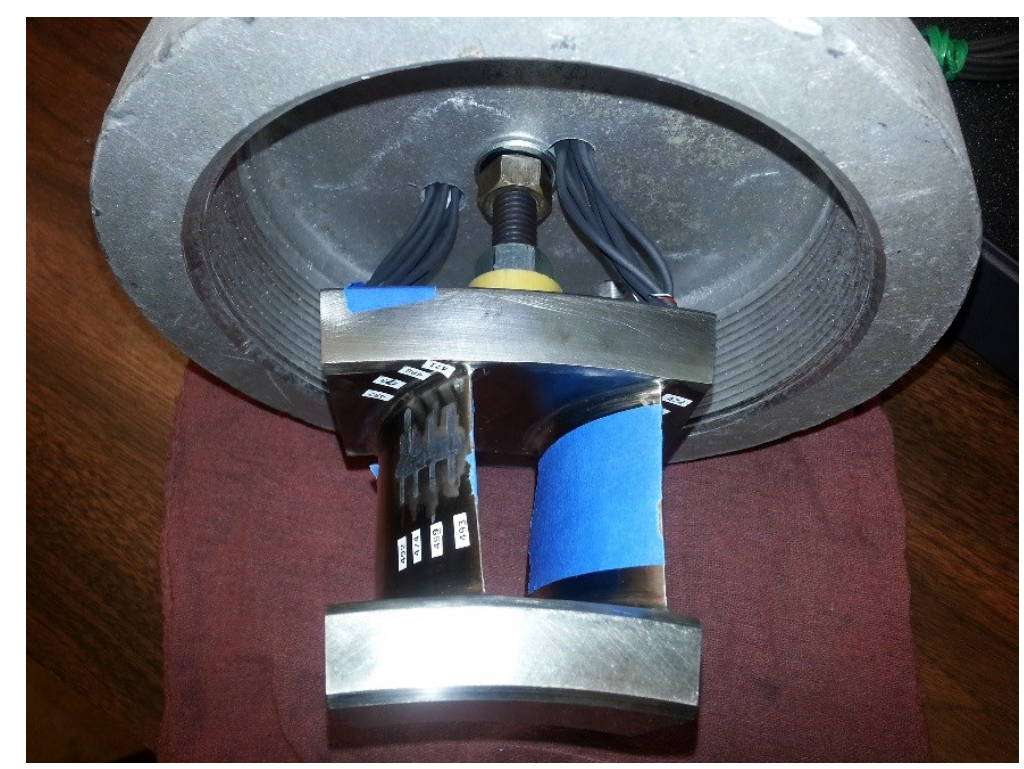

Figure 3.12: Stator installed in cap of calibration chamber, instrumentation cables fed out of cap.

The chamber was pressurized through a range of 0-6 psi, and the calibration information measured at Purdue differed from the data provided by Kulite by as much as $25 \%$ in slope, and this was attributed to the change in response of the Kulite after it was installed in the stator section and potted with hard RTV. Figure 3.13 shows the calibration performed for the stator section, with a gain of 100 on the pressure transducers set in the Precision Filter software. 


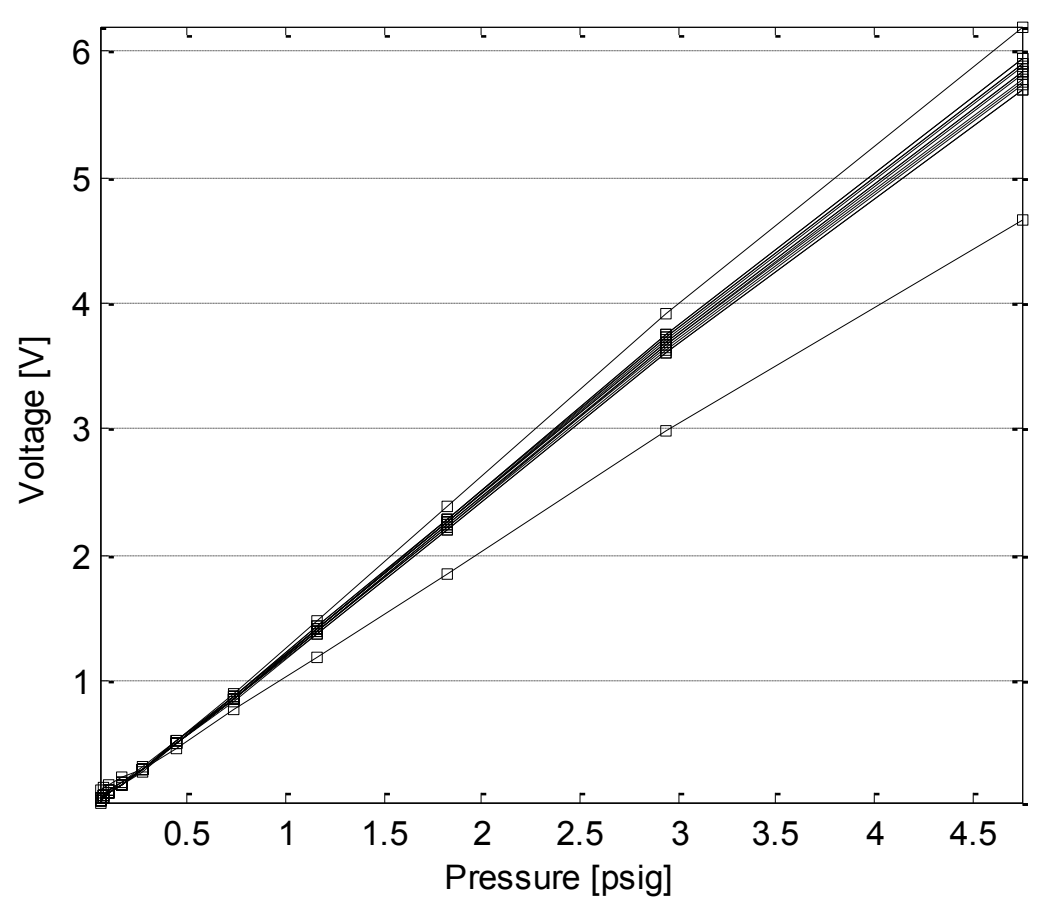

Figure 3.13: Stator Kulite calibration curves for all 15 sensors overlaid.

The stator Kulite data were acquired at several steady operating points, including 74\% corrected speed, with at least 500 revolutions of data for both nominal and high loading conditions. The stator section was also run through several different sweeps through resonance, at both loading conditions, as well as through acceleration and deceleration. Upwards of 1600 revolutions of data are present in each of these sweep runs. The excitation signal and amplification were provided by a Precision Filter 28000 chassis with quantity 4, 28118 full bridge amplification cards installed, each capable of amplifying 8 channels each. No analog filter was applied to the signal by the Precision Filter equipment. Data were acquired with a NI PXI-1073 chassis with one 16-channel NI PXIE-6358 card, with a total bandwidth of 1.25 MS/s per channel for all 16 channels simultaneously. Data was acquired at a sample rate of $300 \mathrm{kHz}$. 


\subsection{Casing Pressure Measurements}

Unsteady casing pressure was measured with flush-mounted fast-response pressure sensors over R2 and in the gap between the upstream and downstream vane rows. A total of 25 Kulite XTL-140 B-Screen Subminiature pressure transducers were installed, with a range of 0-5 psig and a frequency response estimated at $20 \mathrm{kHz}$ by Brossman (2012). The excitation signal and amplification were provided by a Precision Filter 28000 chassis with quantity 428118 full bridge amplification cards installed, each capable of amplifying 8 channels each. Data were acquired with a NI PXI-1073 chassis with two 16-channel NI PXIE-6358 cards, with a total bandwidth of $1.25 \mathrm{MS} / \mathrm{s}$ per channel for all 32 channels simultaneously.

A total of 25 Kulite pressure transducers were installed over the tip of Rotor 2 , extending upstream and downstream of the blade, spanning $120 \%$ of R2 axial chord and $110 \%$ of one blade passage. Prior to installation, all of the transducers were calibrated from 0 to 6 psi in a custom-built aluminum calibration chamber, shown in Figure 3.14.

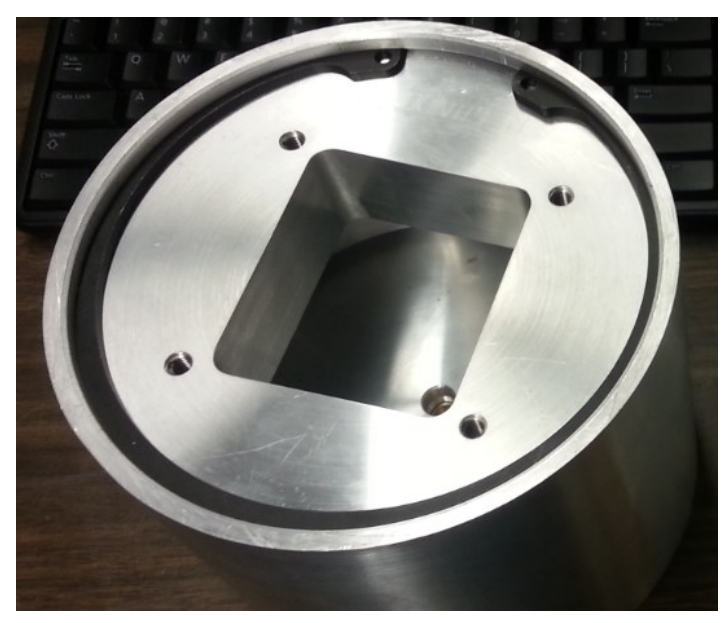

Figure 3.14: Calibration chamber with snap ring and removable bulkhead. 
The pressure transducers were zeroed before installation with a 32-channel Precision Filter amplification system, and the zero-pressure voltage offset was recorded. A photograph of the Kulite window installed in the casing is shown in Figure 3.15.

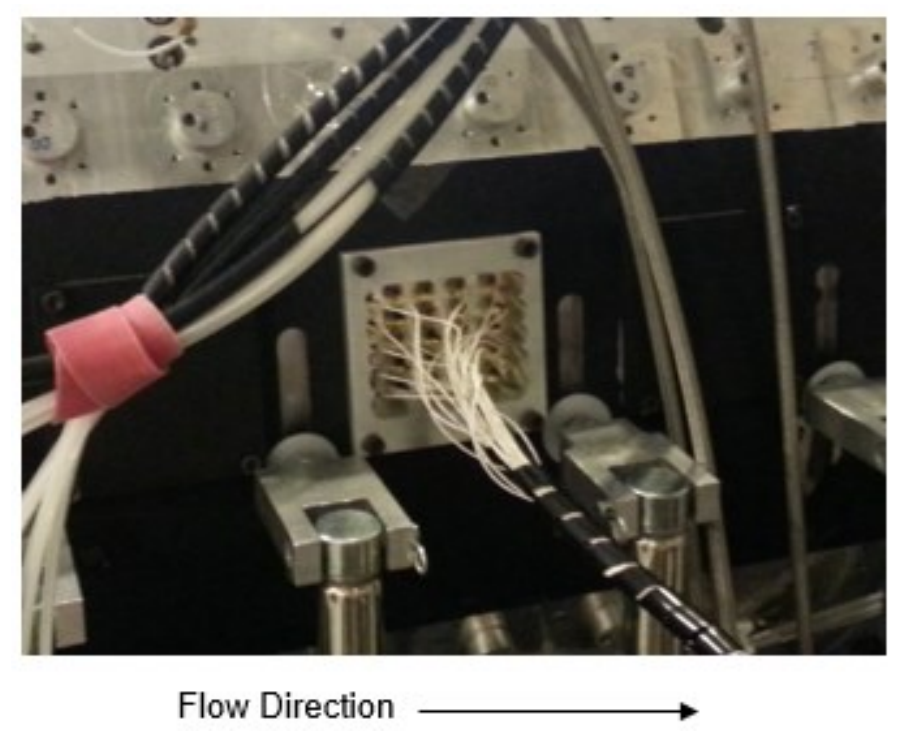

Figure 3.15: Kulites mounted in window block of baseline compressor casing.

Throughout the data acquisition process, many different settings were explored. Sample rates were varied from $150 \mathrm{kHz}$ to $300 \mathrm{kHz}$, compressor ramp speed was varied from 1.8 to $5 \mathrm{RPM} / \mathrm{s}$, accelerations and decelerations through resonance were considered, and both the nominal and high loading conditions were investigated. All data were acquired using LabView while outputting data in binary format and saved to disk.

Depending on the sampling frequency and ramp rate at which the data were acquired, the total amount of time which the sensors were recording data varied from 8 to 30 seconds. Some of the Kulite acquisition runs were at a steady speed, either at the low end or the top end of resonance (3400 RPM or 4000 RPM, for example). The data length 
was characterized by how many revolutions of data were acquired during a given run. On average, throughout a sweep from 3670 to 3750 RPM, about 1800 revolutions of the compressor occurred, giving 1800 "revs" of data for each individual Kulite. On the output shaft of the gearbox, there is a laser tachometer that outputs a $5 \mathrm{~V}$ square wave once per shaft revolution, which corresponds to a specific circumferential blade orientation. This $5 \mathrm{~V}$ square wave was used as a trigger to break up the data from each channel in to manageable vectors, each 1 revolution of the rotor in length. The position at which the $1 /$ rev signal occurs in relation to blade clocking allowed the identification of individual blades in rotor 2 based on the same numbering scheme used by the NSMS data. This allowed direct comparison of NSMS data to pressure data, something that potentially could be used to analyze the aerodynamic characteristics of high or low responding blades.

Figure 3.16 shows the relative positions of the upstream and downstream stators with respect to the Kulite window and sensor grid. The stators were positioned in CL 1 clocking configuration for the duration of testing. Figure 3.17 shows the calibration of all 25 Kulites, overlaid. The calibration was performed with Precision Amplifier gain set to 100. 


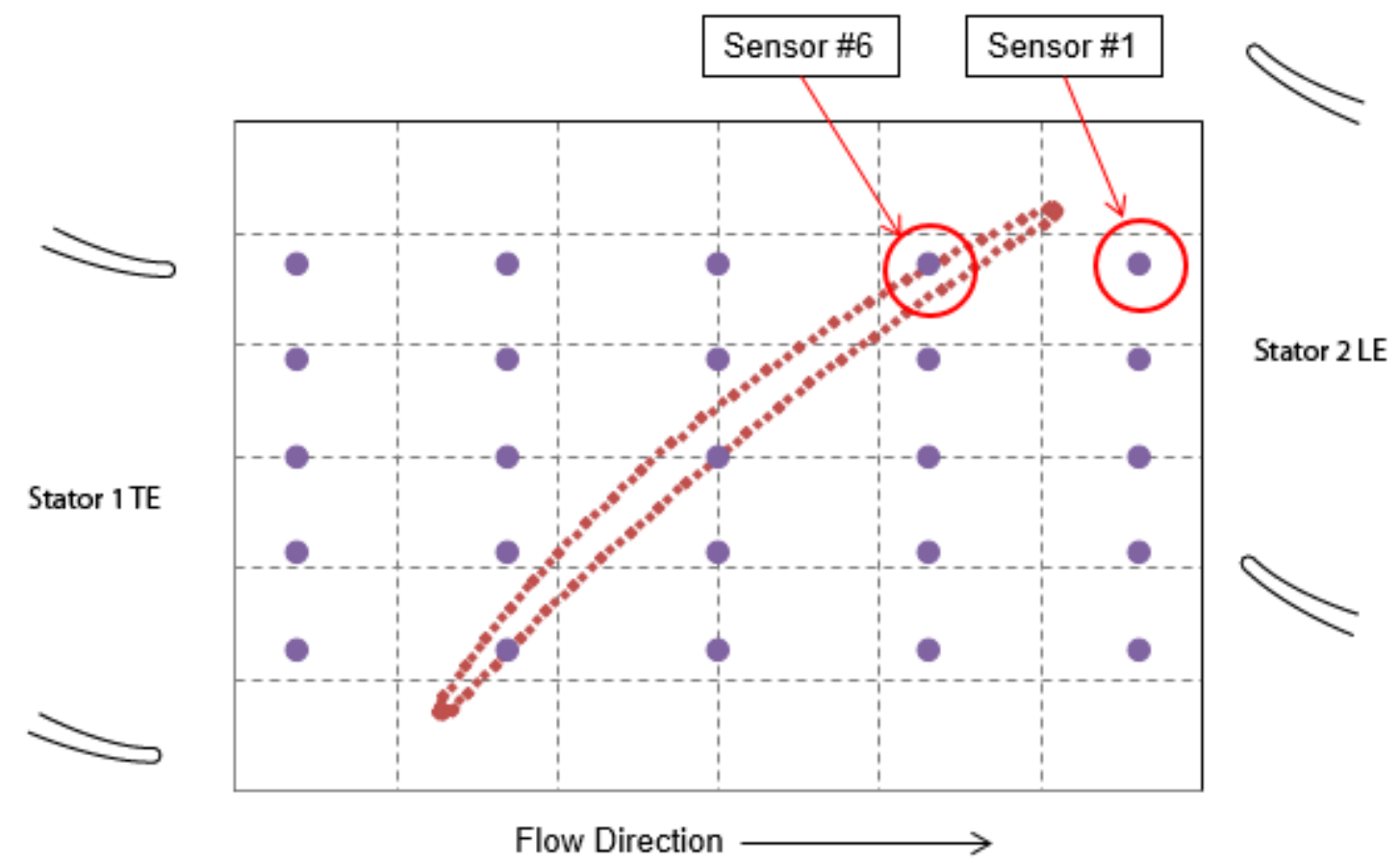

Figure 3.16: Sensor positions relative to R2 blade, with select sensors labeled, CL 1 stator vane clocking configuration.

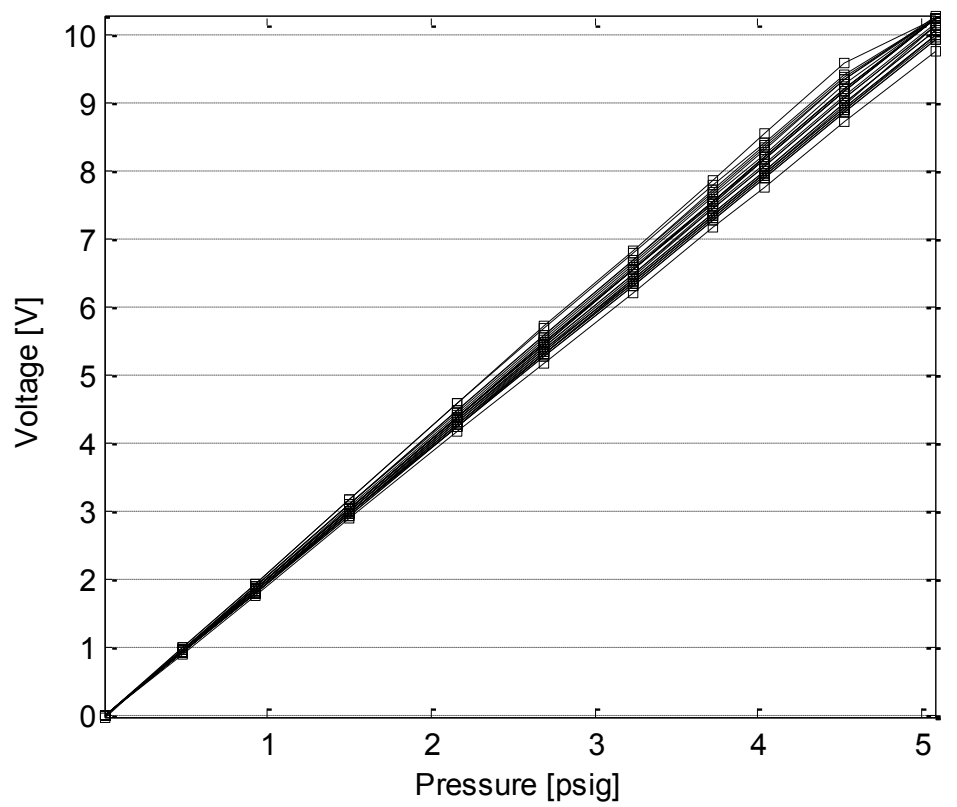

Figure 3.17: Calibration of all 25 sensors installed in casing Kulite window, overlaid. 


\subsection{Thermal Anemometry Measurement}

Thermal anemometry is used to acquire fast response velocity measurements both upstream and downstream of R2. A TSI cross film probe model $1247 \mathrm{~A}-10$ was used in conjunction with TSI IFA 100 constant temperature anemometers to acquire constanttemperature hotwire anemometry measurements. The output from the IFA 100 anemometers was sent to the NI-PXI-1073 chassis, and sampled at $100 \mathrm{kHz}$.

The cross-film probe was calibrated from 100 to $450 \mathrm{ft} / \mathrm{s}$ in steps of $25 \mathrm{ft} / \mathrm{s}$, and from -45 to 45 degrees yaw, in steps of 5 degrees in the Purdue Hotwire Calibration Facility, part of which is shown in Figure 3.18.

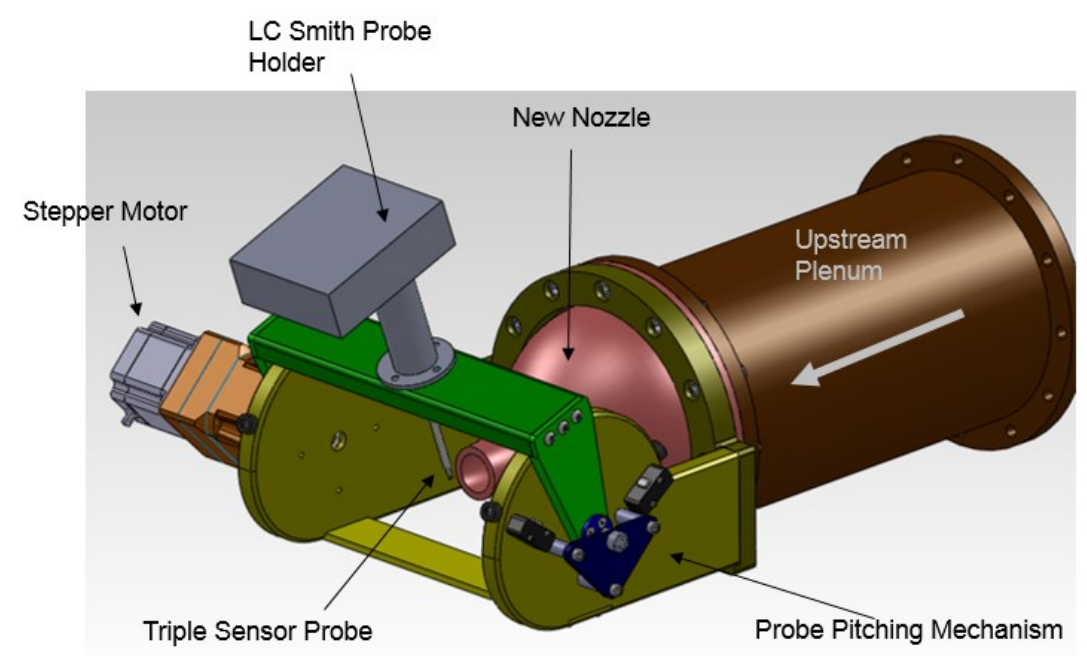

Figure 3.18: Purdue Hotwire Calibration Facility (Morrison, 2013)

The full range calibration of this probe took approximately 70 minutes. The calibration space for the probe in both velocity and yaw angle is shown in Figure 3.19 and Figure 3.20. 


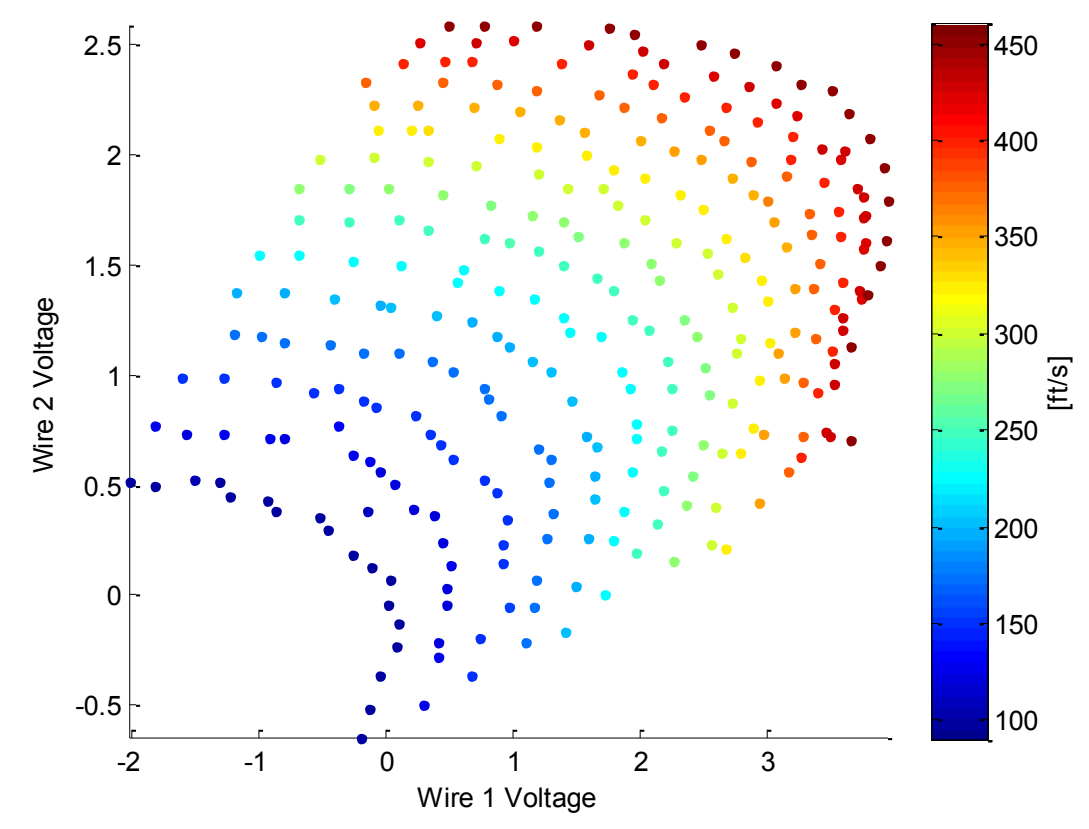

Figure 3.19: Velocity calibration of TSI cross flow film probe.

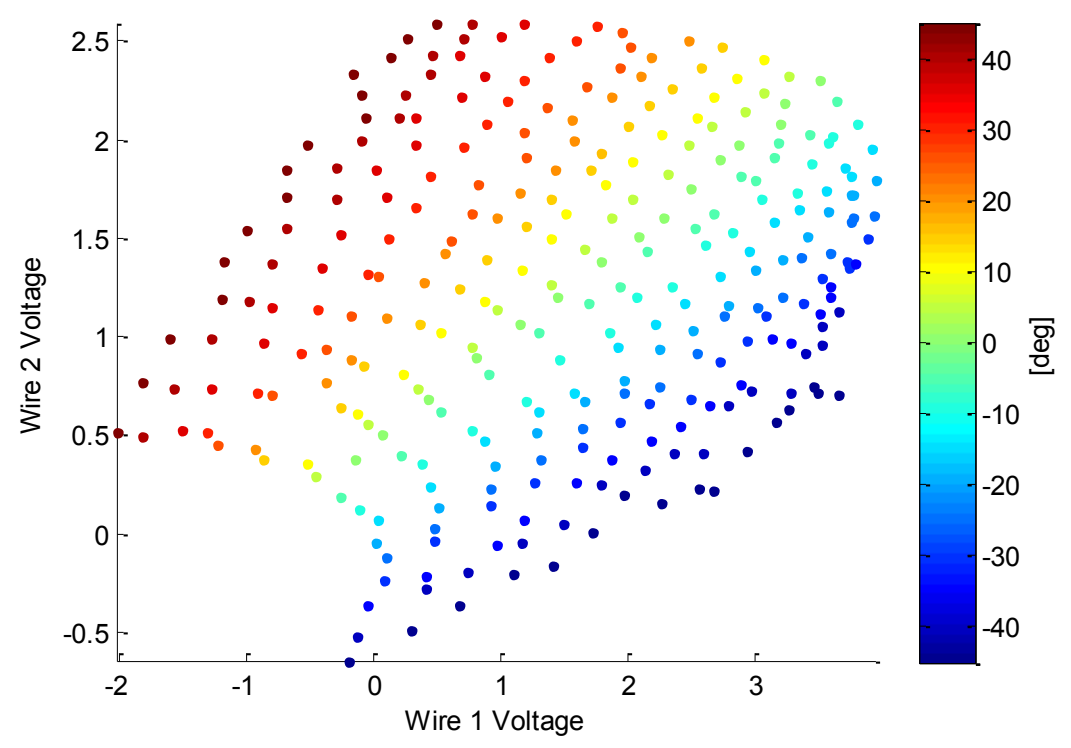

Figure 3.20: Yaw calibration of TSI cross flow film probe.

The IFA 100 amplifier gain was set to 6 and 3 for film 1 and film 2, respectively. The output voltages are not only a function of flow velocity and angle, but also a weak function of stagnation temperature and static density. Since the stagnation temperature and 
static density inside the compressor will be significantly different than conditions for the probe calibration, the measured voltages will need to be adjusted in order to compensate for these offsets. Temperature and density corrections were applied in a similar fashion to hotwire data previously acquired with this hotwire system, explained in detail by Morrison (2013). The wire voltages are assumed to follow Eqn. 3-1, where $T_{w}$ is the wire temperature (which is set by the resistances prescribed by TSI for this particular probe to equal $482^{\circ} \mathrm{F}$ ). $T_{o}$ is the temperature of the flow (measured by total temperature rakes), $E$ is wire voltage, and $K$ is a constant.

$$
\frac{E^{2}}{\left(T_{w}-T_{o}\right)}=K
$$

According to Bruun (1995), since $K$ is a constant for each wire, the wires can be solved for an adjusted voltage based on the flow temperature and adjusted corresponding to the wire temperature and the temperature at which the probe was calibrated and at which it was actually tested within the compressor.

$$
E_{\text {adjusted }}=E_{\text {test }} \sqrt{\frac{T_{w}-T_{0_{c a l}}}{T_{w}-T_{0_{\text {test }}}}}
$$

The probe was calibrated through a range of densities from approximately 0.071 $\mathrm{lbm} / \mathrm{ft}^{3}$ to $0.081 \mathrm{lbm} / \mathrm{ft}^{3}$, at 4 separate velocities, and the probe voltage was a linear function of static density as shown in Figure 3.21 and Figure 3.22. 


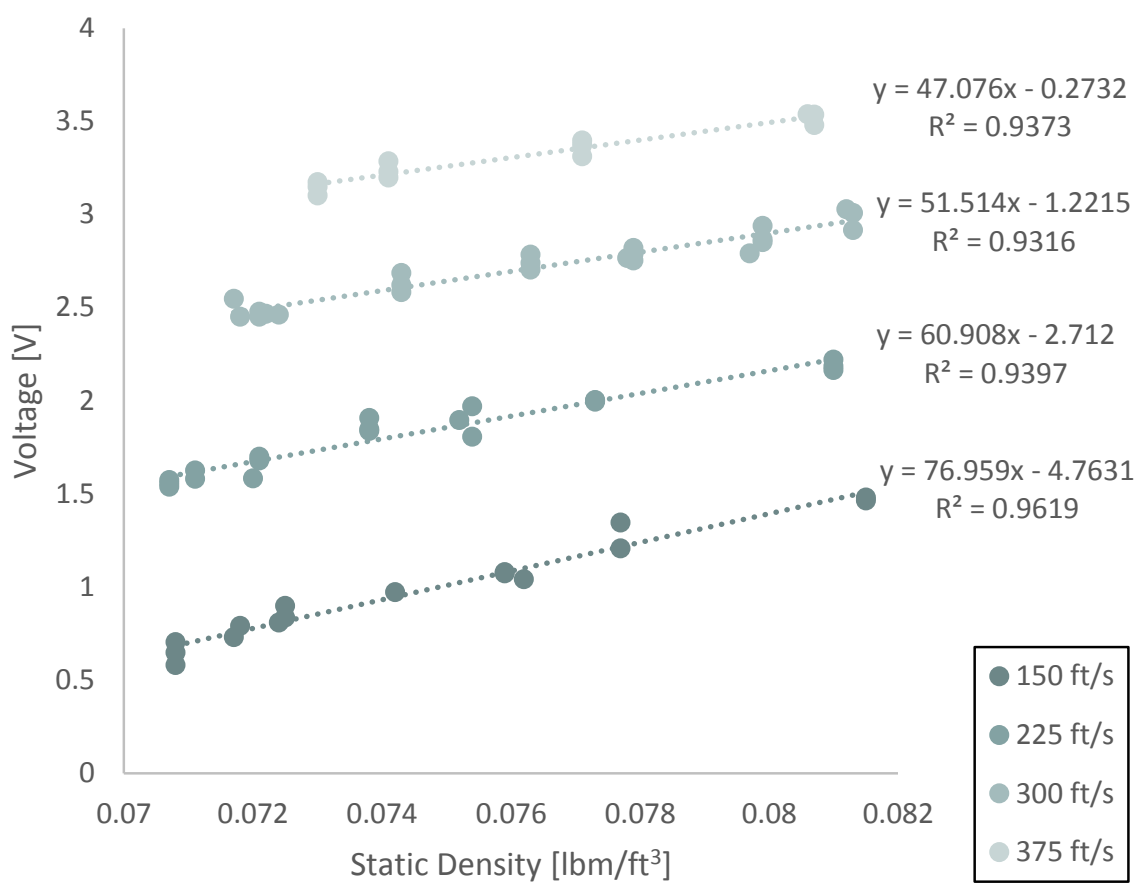

Figure 3.21: Density calibration for TSI 1247A-10 Cross Film probe, Wire 1.

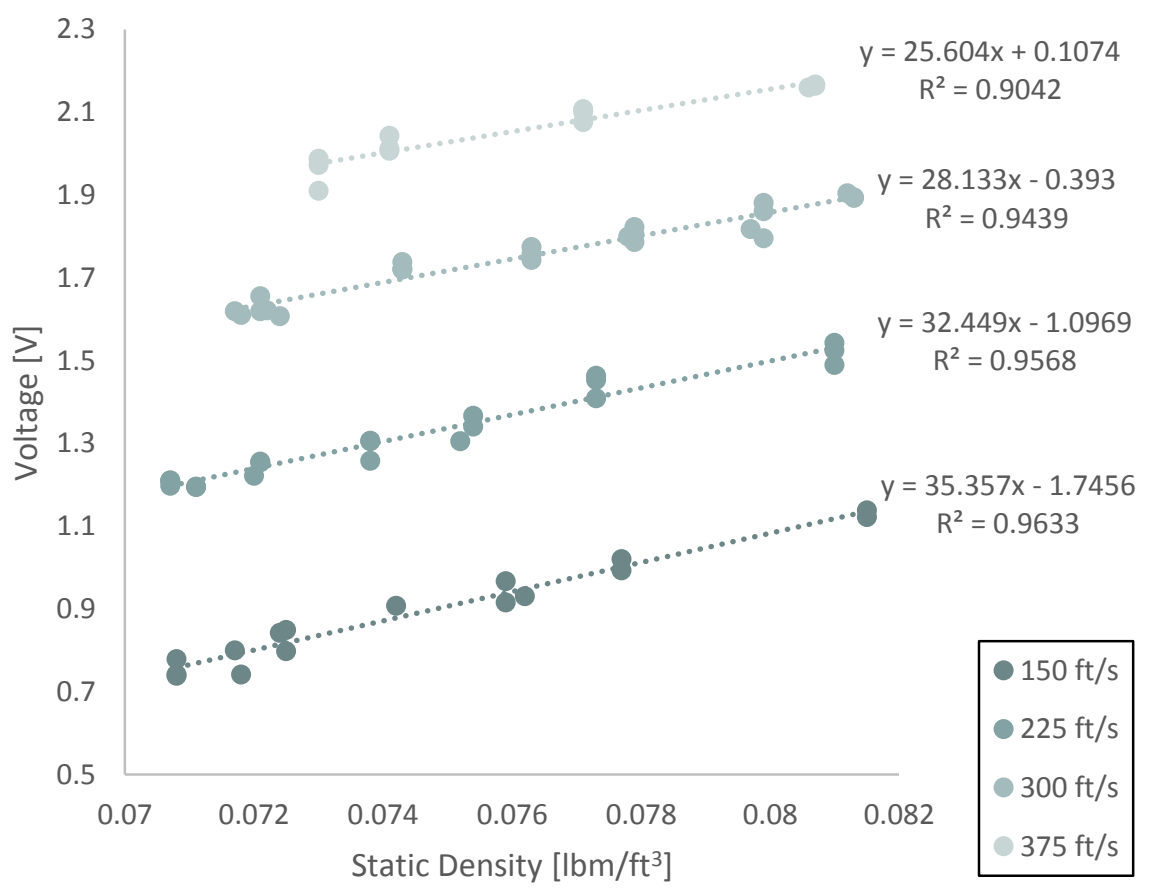

Figure 3.22: Density calibration for TSI 1247A-10 Cross Film probe, Wire 2. 
Eqn. 3-3 was then used to correct for density changes, where $\partial E / \partial \rho$ was found to be a function of velocity, ranging between $25.6 \mathrm{~V} / \mathrm{lbm} / \mathrm{ft}^{3}$ to $77 \mathrm{~V} / \mathrm{lbm} / \mathrm{ft}^{3}$ depending on the wire.

$$
E_{\text {adjusted }}=E_{\text {test }}+\frac{\partial E}{\partial \rho}\left(\rho_{\text {test }}-\rho_{\text {cal }}\right) \quad \text { Eqn. 3-3 }
$$

Since the density during the calibration changed slightly, and the jet temperature changed over 2 degrees, the calibration was normalized to a density of $0.070 \mathrm{lbm} / \mathrm{ft}^{3}$ and temperature of $100^{\circ} \mathrm{F}$ using Eqn. 3-2 and Eqn. 3-3 and referencing the correct value for $\partial E / \partial \rho$ depending on the recorded velocity. It is to these set values that the test data was then compared to with the lookup functions. The MATLAB function ScatteredInterpolant was used to create a lookup grid based on velocity and yaw information from the calibration. Steady data recorded from total temperature and total pressure rakes, combined with static pressure recorded at the axial position of the measurement were used in conjunction with REFPROP, a thermodynamic table, and relative humidity measurements to calculate density and total temperature inside the compressor at the axial and span location that the hotwire probe was located at.

Since density is a function of velocity, an iterative lookup process was used to correct for density. First, an assumed value for $\partial E / \partial \rho$ was used to calculate velocity based on the calibration for a steady operating point. The mean of the calculated velocity was found, and $\partial E / \partial \rho$ was calculated based on a linear line fit for each wire, as shown in Figure 3.23 and Figure 3.24. 


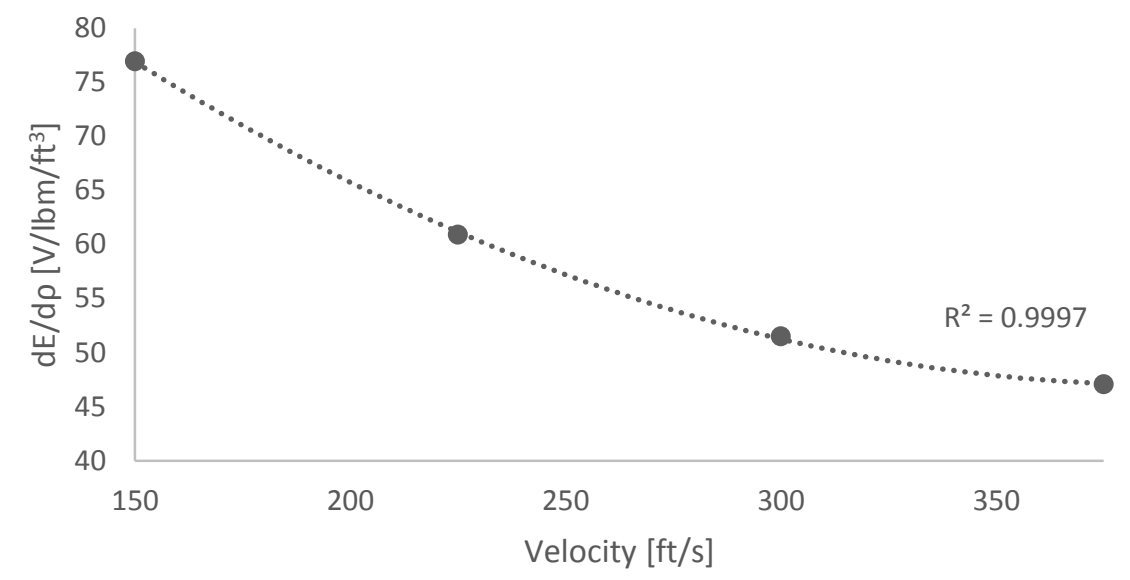

Figure 3.23: Change in density sensitivity as a function of velocity, Wire 1.

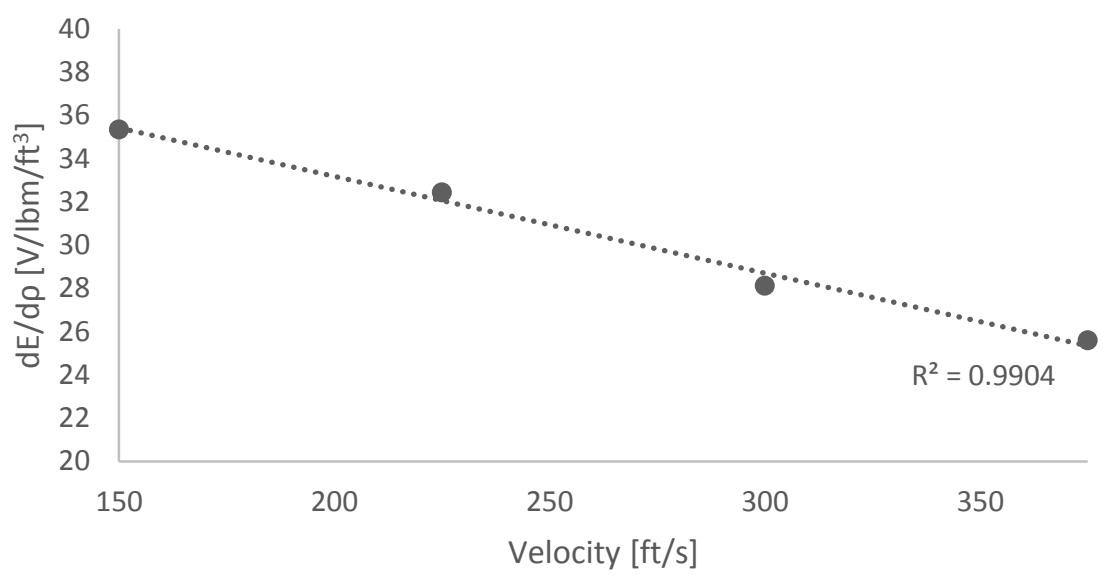

Figure 3.24: Change in density sensitivity as a function of velocity, Wire 2.

Figure 3.25 is a depiction of probe orientation with respect to the velocity triangles at $\mathrm{S} 1$ exit and $\mathrm{R} 2$ exit. The probe was oriented to face the mean flow direction in the absolute reference frame: $12^{\circ}$ at $\mathrm{S} 1$ exit and $44^{\circ}$ at $\mathrm{R} 2$ exit, where positive angles are in the direction of rotor rotation. 


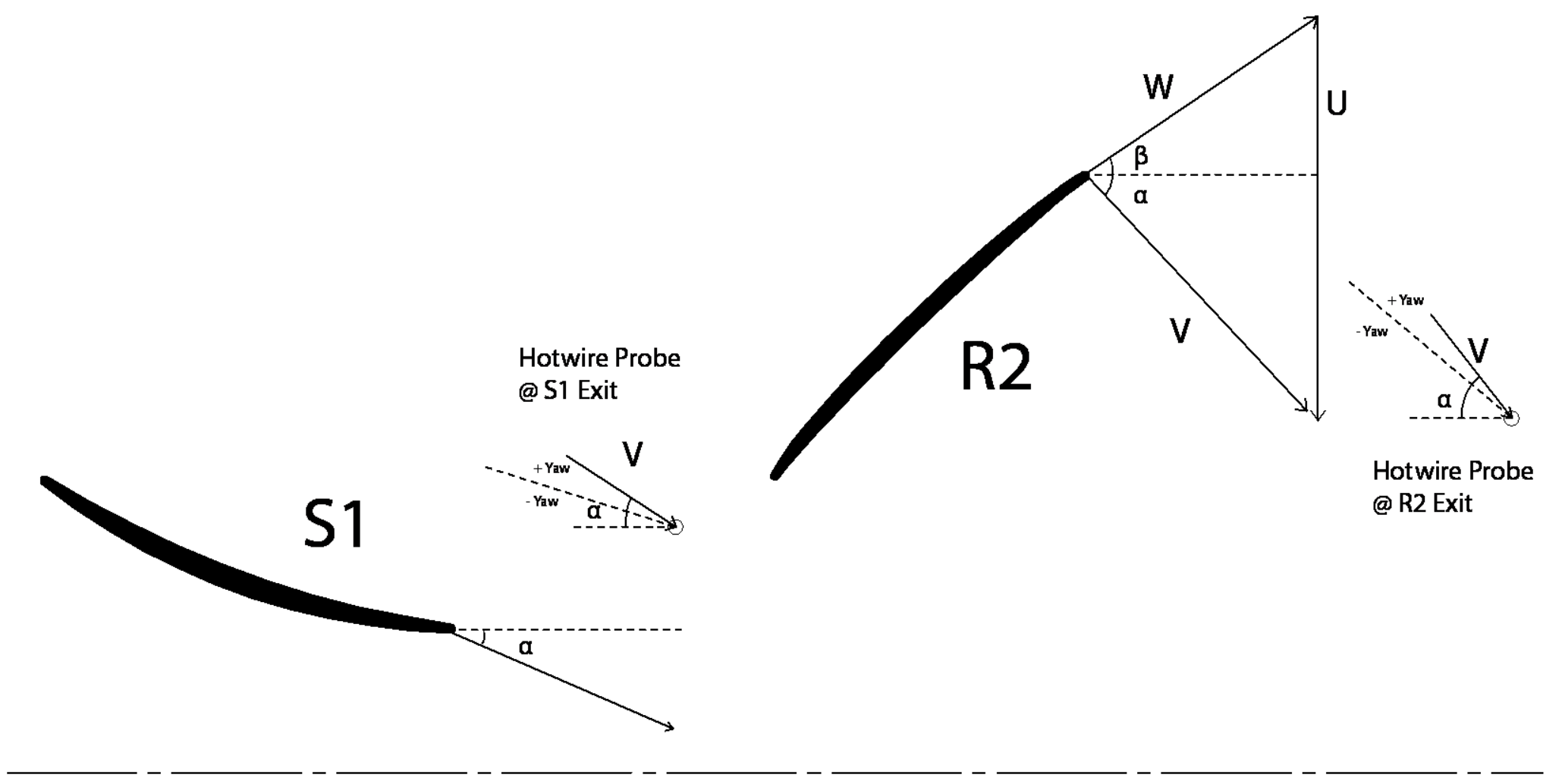

Figure 3.25: Illustration of angle decomposition used to resolve velocity components for both hotwire axial locations. Flow from right to left, as seen by probe. 


\section{CHAPTER 4. CHARACTERIZTION OF FORCING FUNCTIONS \& ROTOR RESPONSE}

\subsection{Compressor Operating Conditions}

This chapter presents the data acquired at the 44 EO Campbell Diagram crossing of the R2 1T vibratory mode. Data were acquired at 2 loading conditions with a focus at the $50 \%$ and $80 \%$ span locations.

\subsubsection{Compressor Operation}

To capture the aerodynamics at the speed where resonance occurs, data were acquired at corrected conditions $(74 \%$ corrected speed) on days where the ambient conditions placed the required mechanical speed outside of the resonance speed. According to Eqn. 4-1, the ambient temperature had to be in the range of approximately $40^{\circ} \mathrm{F}$ to $52^{\circ} \mathrm{F}$.

$$
N_{c}=\frac{N_{m}}{a_{o}}
$$

Thus, the aerodynamics at $74 \%$ corrected speed when the rotor was not vibrating were captured. The calculation of corrected speed takes into account the ambient temperature and relative humidity, and uses REFPROP, a thermodynamic table, to calculate the true corrected speed and massflow and is detailed in Berdanier et al. (2014). 
Due to the close proximity of $1 \mathrm{~T}$ vibratory modes of each rotor, tip timing was used to map out the $1 \mathrm{~T}$ response for all 3 rotors. Figure 4.1 shows the frequency range of each rotor assembly. There is a gap between approximately 3640 and 3680 RPM in which the compressor can operate free of rotor and stator vibration modes, and it is within this region that the $74 \% \mathrm{~N}_{\mathrm{c}}$ points were taken.

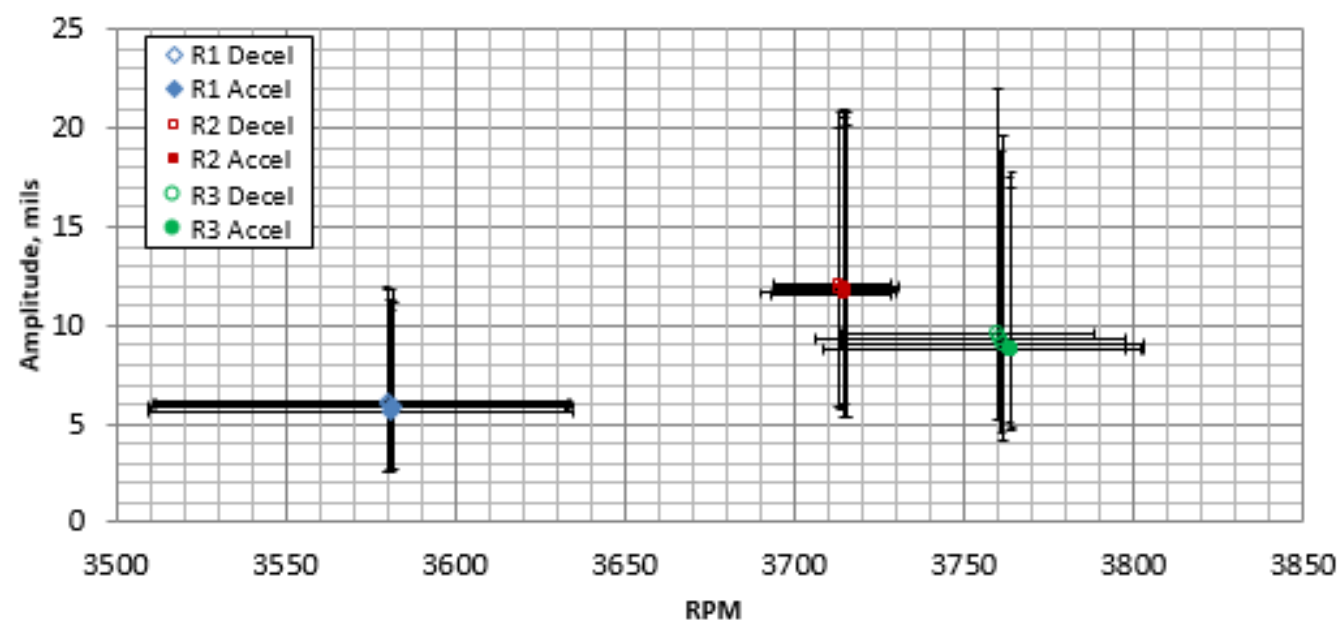

Figure 4.1: NSMS data showing RPM band and relative amplitude of R1, R2, and R3 1T crossings.

At the beginning of every run, the compressor drive system is allowed to warm up over a period of approximately one hour while instrumentation and mechanical systems are checked. Once spinning, the compressor is slowly ramped up to $500 \mathrm{RPM}$, where it is allowed to dwell to bring the temperature of rotating hardware up to a safe range. At this point, the throttle is left wide open, and there is no significant loading on the blading. Once the compressor is sped up to the desired corrected speed, the throttle is then set to the loading point specified by compressor $T P R / \dot{m}$ and held in this position while data are acquired for that operating point on the compressor map. During sweeps though resonance, 
the throttle is set to the desired loading position at the lowest steady RPM and held at that same position through the accelerating and decelerating sweep.

\subsubsection{Steady Performance}

For each point on a speed line, the automated LabView program that controls performance data acquisition on the 3-stage compressor indexes the vanes past the steady instrumentation rakes, and an area-averaged total pressure ratio is calculated. This is based on circumferentially averaging 4 total pressure rakes at the Aerodynamic Interface Plane (AIP) and averaging the single exit rake over one vane pitch at the rig exit. This provides a very accurate measurement of total pressure ratio as it takes into account the stator wakes that may effect a measurement of total pressure ratio taken in just one circumferential location. This is also how the two speed lines were generated in Figure 4.2.

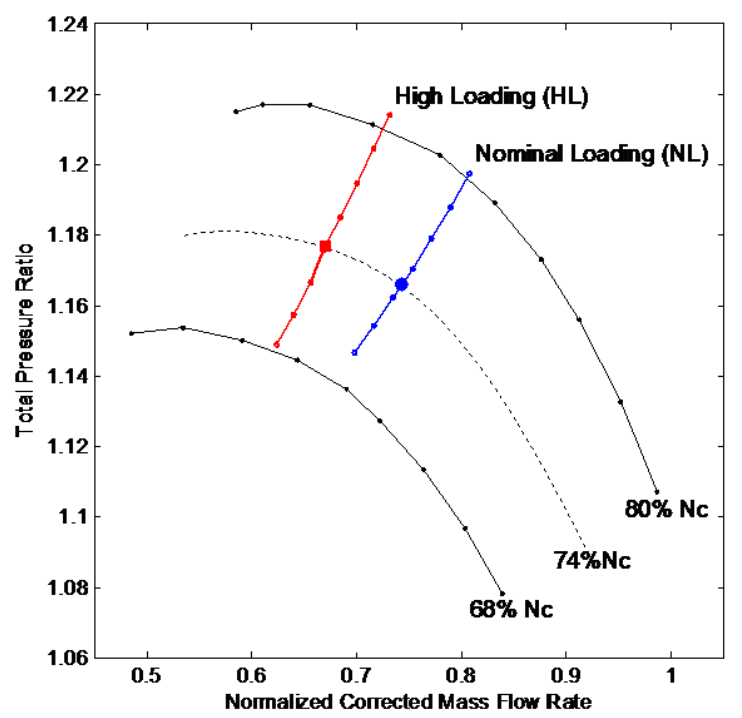

Figure 4.2: Compressor map showing regions of data acquired during aeromechanics studies. 
Boundary conditions were documented so that this dataset could be used for code validation. Mid-span turbulence intensity at the compressor inlet was approximately $2 \%$, as measured by a hotwire. The inlet total temp profile is shown in Figure 4.3. It would be common to expect uniform inlet $\mathrm{T}_{\mathrm{o}}$, but the temperatures near the casing are higher for these experiments. This is associated with heat conduction in the casing. As work is done on the flow, the flow temperature increases in the back stages, and heat is transferred to the casing. Conduction of heat from the rear of the casing to the front part of the casing results in a casing temperature that is warmer than the air temperature in the front stages. Surfacemounted thermocouples placed over each blade row show the casing temperature and can be used as an isothermal boundary condition in CFD simulations, shown in Figure 4.4. This results in higher temperatures in the flow in the outer portion of the span at the compressor inlet.

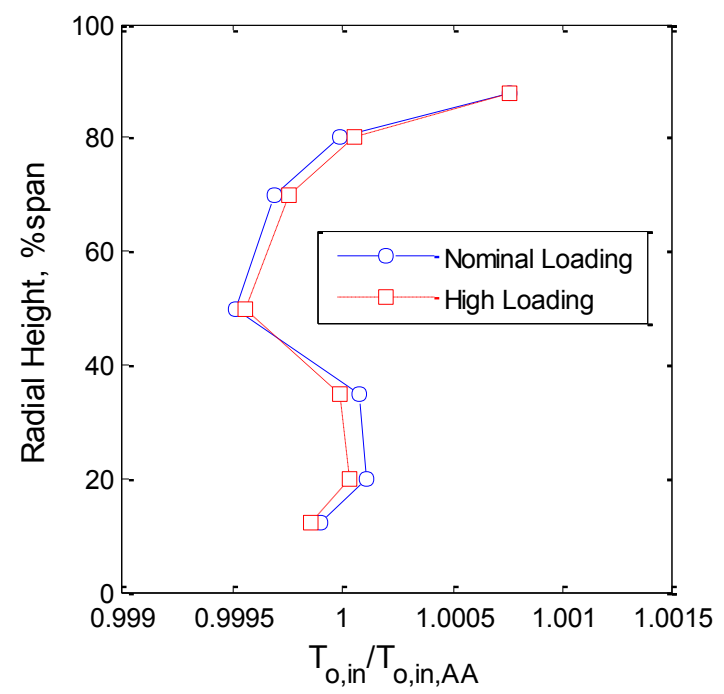

Figure 4.3: Inlet total temperature profile at $74 \% \mathrm{~N}_{\mathrm{c}}$.

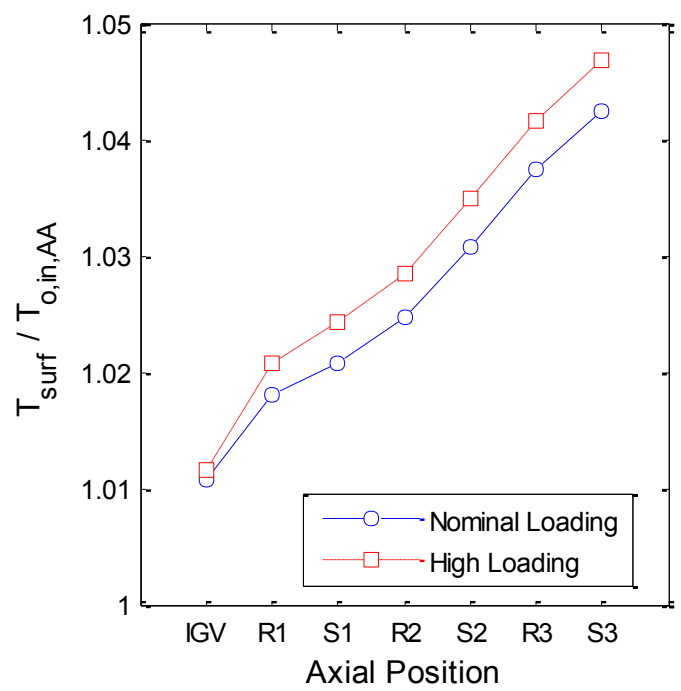

Figure 4.4: Casing surface temp as a function of axial position at $74 \% \mathrm{~N}_{\mathrm{c}}$. 
The detailed compressor inlet total pressure profile was measured using a miniature Kiel head total pressure probe mounted to a radial traverse mechanism, and data were acquired at $74 \% \mathrm{Nc}$ for Nominal Loading (NL) and High Loading (HL), as shown in Figure 4.5. Boundary layer effects are noticeable in Figure 4.5, where the total pressure ratio near the hub and tip is, in general, lower than mid-span values, especially at the casing wall. The stronger casing wall boundary layer is a result of the long inlet duct, as the hub boundary layer only begins to form at the front of the compressor nosecone.

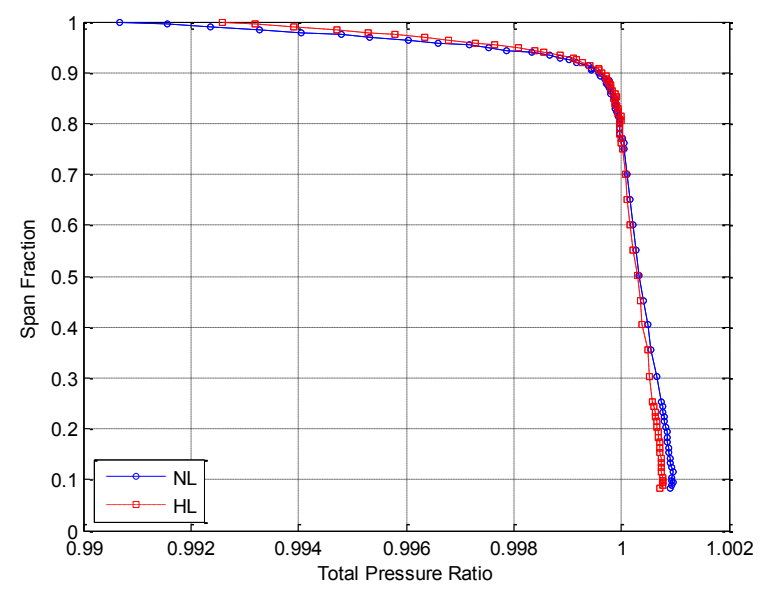

Figure 4.5: Inlet pressure profile at $74 \% \mathrm{~N}_{\mathrm{c}}$ for $\mathrm{NL}$ and $\mathrm{HL}$.

Figure 4.6 and Figure 4.7 show the radial total pressure and total temperature distribution in the embedded stage where most of the data for this project was taken. This is important as well for calculating CFD boundary conditions, as well as providing important temperature and density flow information for use in hotwire voltage corrections at the same measurement locations. The total pressure and total temperature measurements are normalized by the area-averaged total pressure and total temperature at the AIP or 
Aerodynamic Interface Plane (station 0). At high loading Rotor 2 is doing more work on the flow, and thus, $T_{0}$ is elevated rather uniformly across the span.
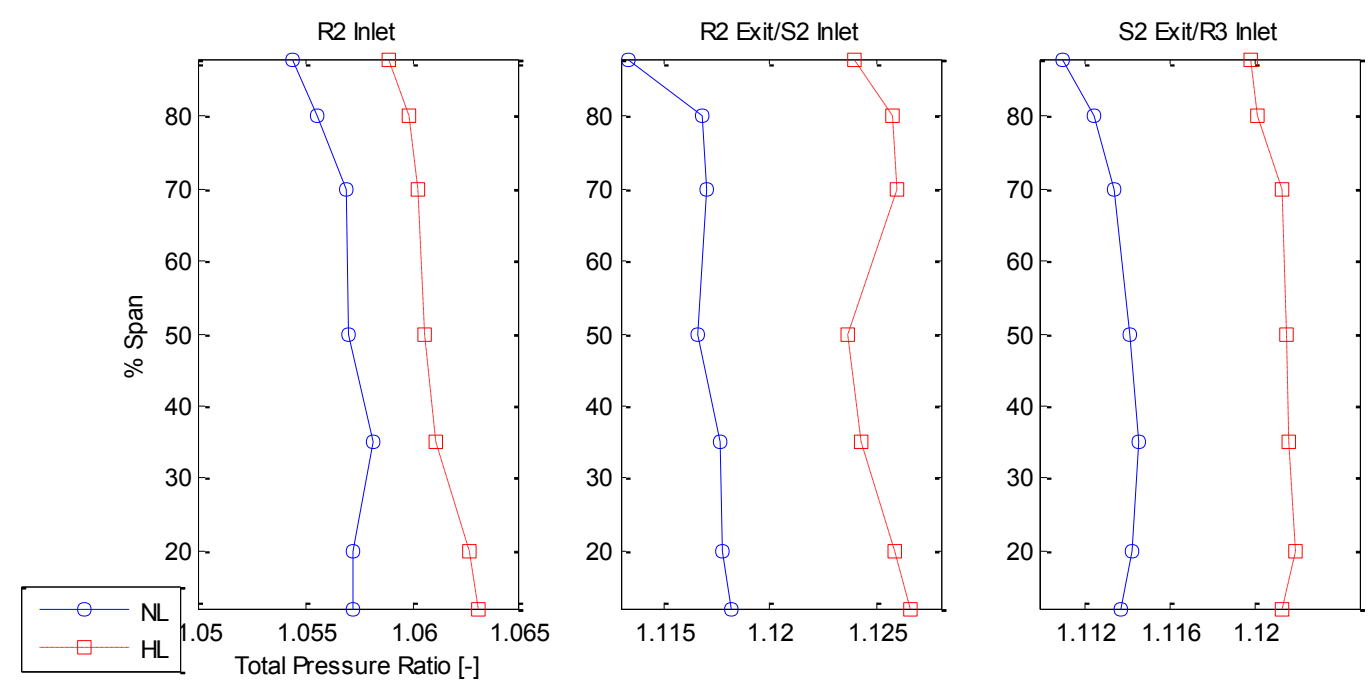

Figure 4.6: Steady total pressure measurements at three axial locations.
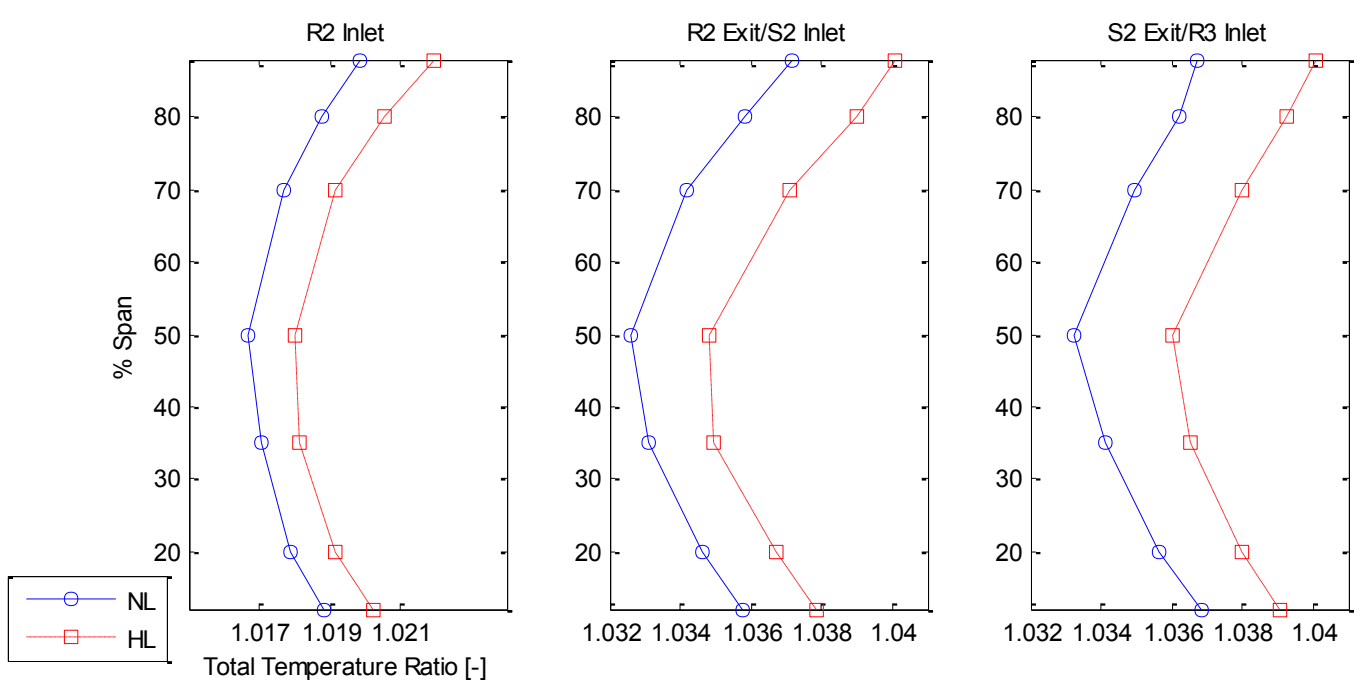

Figure 4.7: Steady total temperature measurements at three axial locations. 


\subsection{Forcing Functions for 44 EO R2 1T Resonance}

This section will discuss both the vortical forcing function from S1 wakes and the potential forcing function from S2 potential fields that were acquired through the use of total pressure rakes, static pressure taps, and thermal anemometry.

\subsubsection{Vortical Forcing Function}

The Stator 1 wakes provide the vortical forcing function to R2 vibrations for the 44EO Campbell diagram crossing. The wakes were characterized with both pneumatic total pressure rakes and thermal anemometry. A 50-point vane traverse (every $2 \% \mathrm{VP}$ ) with a 7-element total pressure rake was conducted at 74\% corrected speed for both Nominal and High Loading cases as shown in Figure 4.8 and Figure 4.9. The scale of the ordinate is held constant for both loading conditions so the wake shapes can be compared. At nominal loading, there is little difference between the $50 \%$ span and $80 \%$ span wake shapes. At high loading, the $80 \%$ span wake is approximately $40 \%$ wider and $38 \%$ deeper than the $50 \%$ span wake. Both wakes are wider and deeper than those measured at nominal loading. The deeper and wider wakes help contribute to the higher blade responses measured in the NSMS tip timing data. 


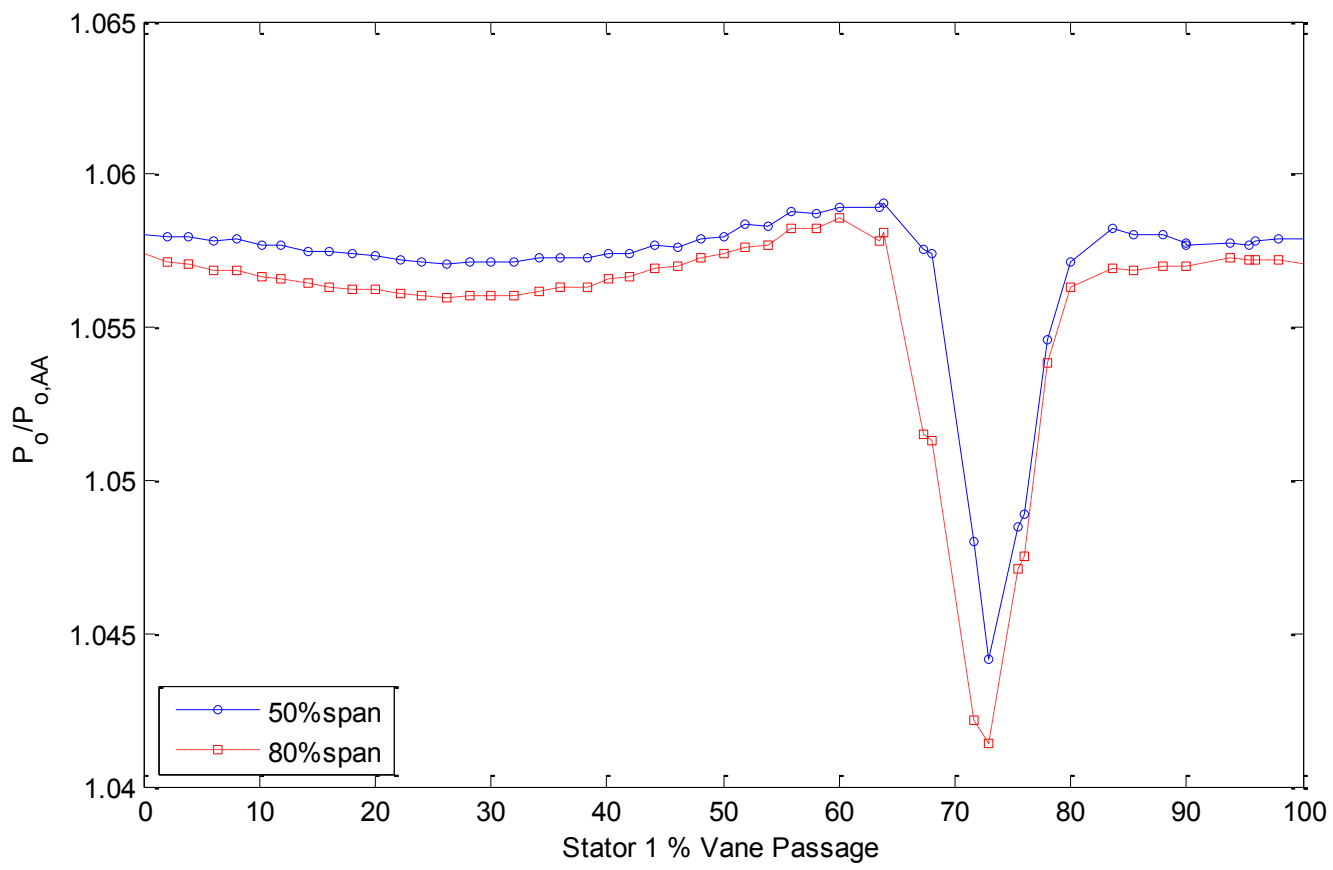

Figure 4.8: S1 50-point wake traverse at Nominal Loading, 74\% $\mathrm{N}_{\mathrm{c}}$.

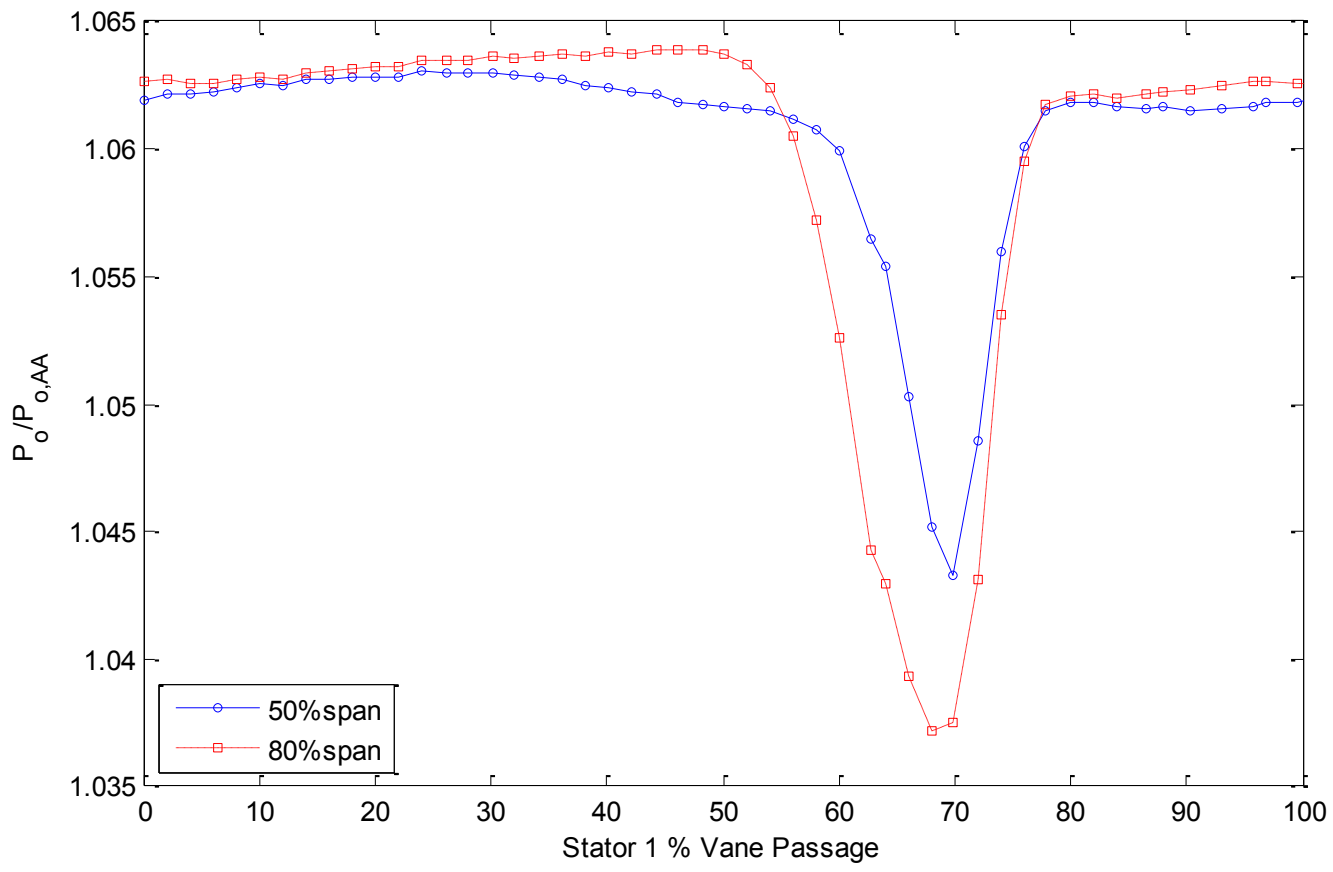

Figure 4.9: S1 50-point wake traverse at High Loading, 74\% $\mathrm{N}_{\mathrm{c}}$. 
A Fourier transform of the wake shapes is shown in Figure 4.10 and Figure 4.11. The $\mathrm{x}$-axis frequency has been normalized by the compressor revolution frequency. The frequency component associated with $44 / \mathrm{rev}$ is responsible for driving the forced response $\mathrm{R} 2$ vibration at the $44 \mathrm{EO}$ crossing. Ten harmonics are included. The magnitude of the Fourier decomposition associated with the vane pass frequency for nominal loading is smaller than the second harmonic. The high loading results show that the magnitudes of the Fourier components decrease with increasing harmonics. This is because the shape of the nominal loading wake is sharper, more like an impulse than a sine wave, and it has distributed energy into the higher harmonics. The magnitude of the first harmonic of vane pass frequency at high loading is three times larger than that at nominal loading at $80 \%$ span. 


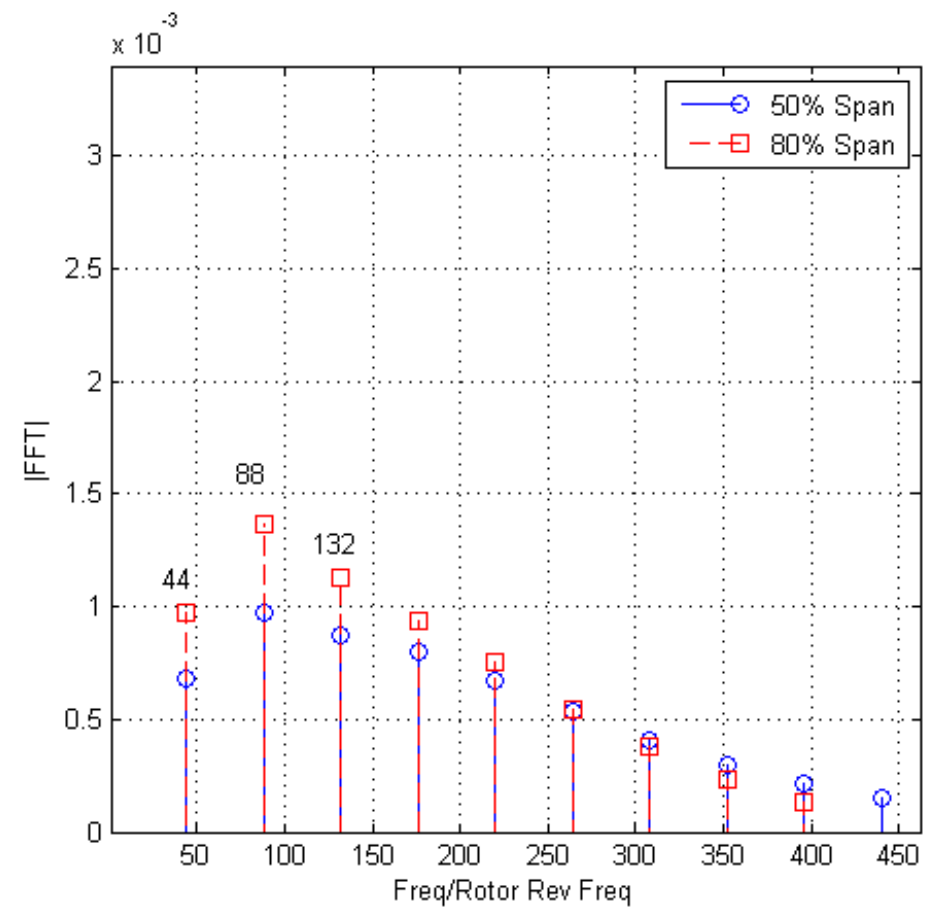

Figure 4.10: FFT of S1 wake from Po rake, NL.

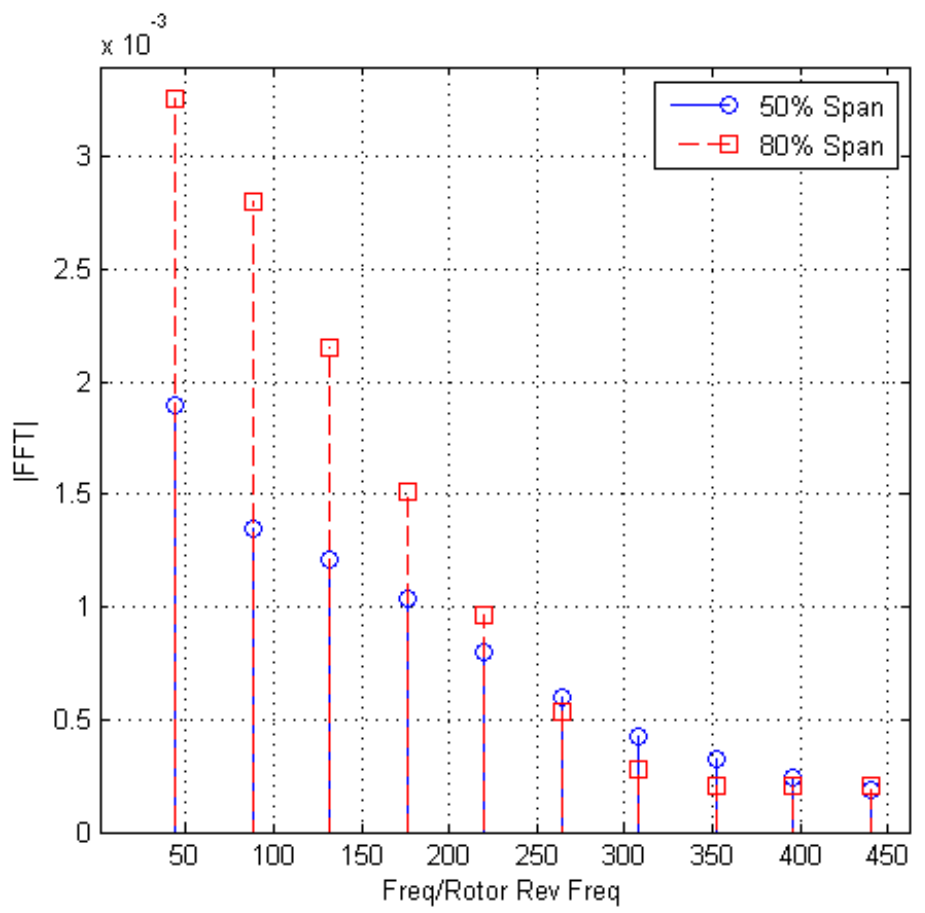

Figure 4.11: FFT of S1 wake from Po rake, HL. 
The time-averaged velocity wakes obtained by thermal anemometry are shown in Figure 4.12 and Figure 4.13 and follow the same trends in the total pressure rake data, with $66 \%$ deeper and $21 \%$ wider wakes seen at high loading (at $80 \%$ span) as compared to nominal loading. The plots were created by computing an ensemble average of 500 revolutions of data at each point, and taking the mean of the ensemble average. There is a significant difference between wakes at different span locations. The wake at $80 \%$ span at nominal loading is $26 \%$ deeper and $70 \%$ wider than $50 \%$ span, and the wake at $80 \%$ span at high loading is negligibly deeper (less than $2 \%$ ) but $81 \%$ wider. As in Figure 4.12 , there is a shift in the center of the wake trough, from approximately 53\% VP to $57 \% \mathrm{VP}$, and this is similarly in Figure 4.13, as well as an overall shift to lower \%VP from higher loading and a lower velocity corresponding to a lower overall compressor massflow.

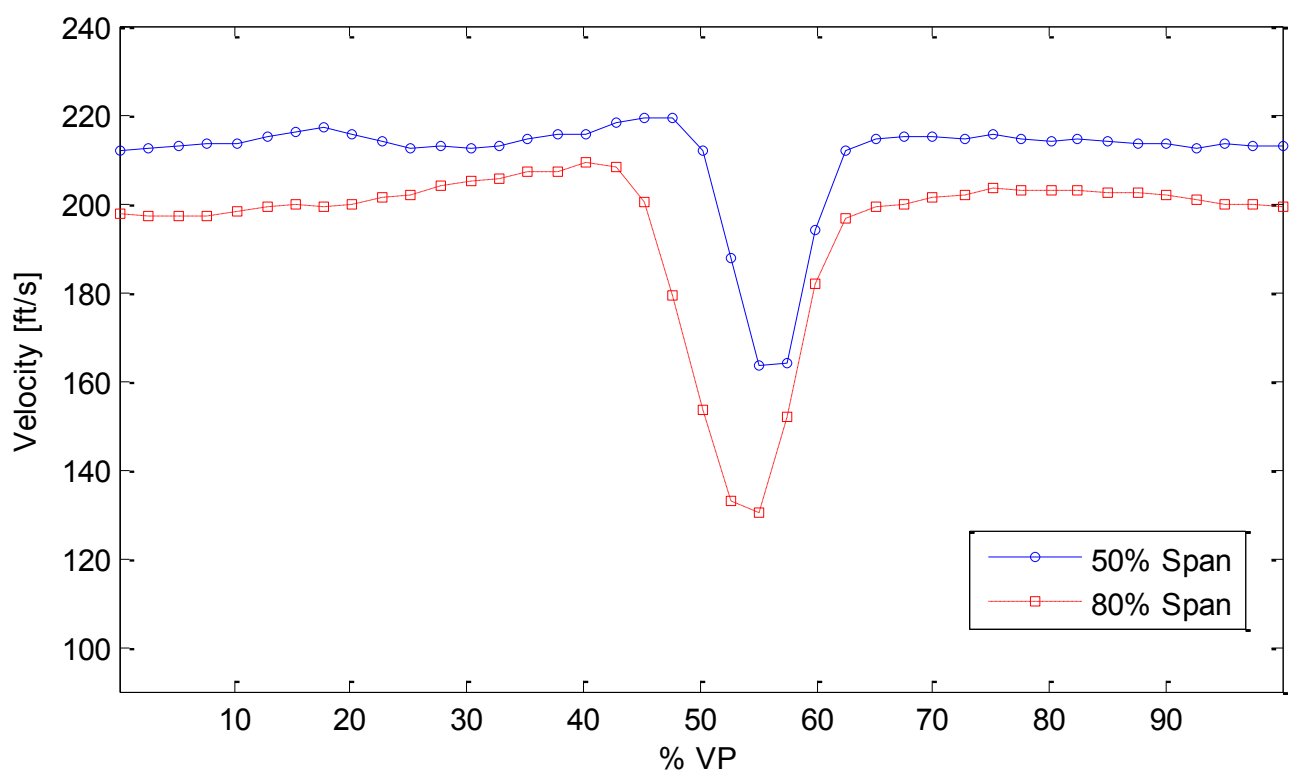

Figure 4.12: 40 point hotwire traverse of absolute velocity at S1 Exit, NL. 


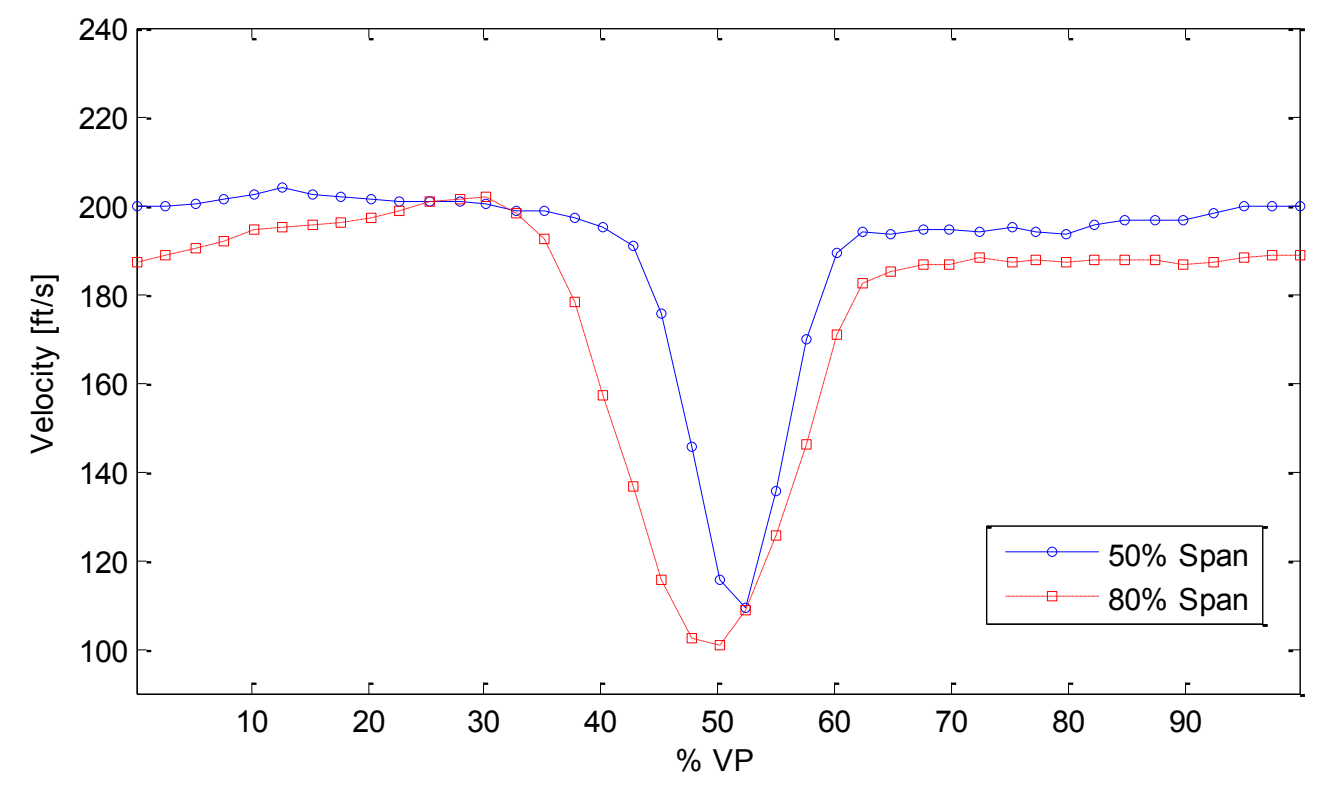

Figure 4.13: 40 point hotwire traverse of absolute velocity at S1 Exit, HL.

Figure 4.14 and Figure 4.15 show the first 10 harmonics of the velocity wake Fourier transform for 50\% and $80 \%$ span at NL and HL. The FT of the hotwire rakes reveals a similar picture to the FTs of the $\mathrm{P}_{\mathrm{o}}$ data, showing the second order harmonic of the wake higher than the $1^{\text {st }}$ harmonic for NL, again, due to the sharper impulse-like wake shape. At HL the wake shape is broader than at NL and the spectra shows a decrease in magnitude with increasing frequency. At $80 \%$ span, the $1^{\text {st }}$ harmonic of the stator wake is $125 \%$ larger at HL than at NL due to the larger velocity deficit.

The largest difference between the FT of the $\mathrm{P}_{\mathrm{o}}$ wakes compared to the velocity wakes is the larger difference in FT magnitudes between $50 \%$ and $80 \%$ span at NL and the fact that the $3^{\text {rd }}$ harmonic of the velocity wake at $50 \%$ span is the largest of all harmonics at NL. This is due to the very small velocity wake shape in comparison to the overall vane 
passage width, but may also be partly due to the coarser traverse used for the thermal anemometry traverse.

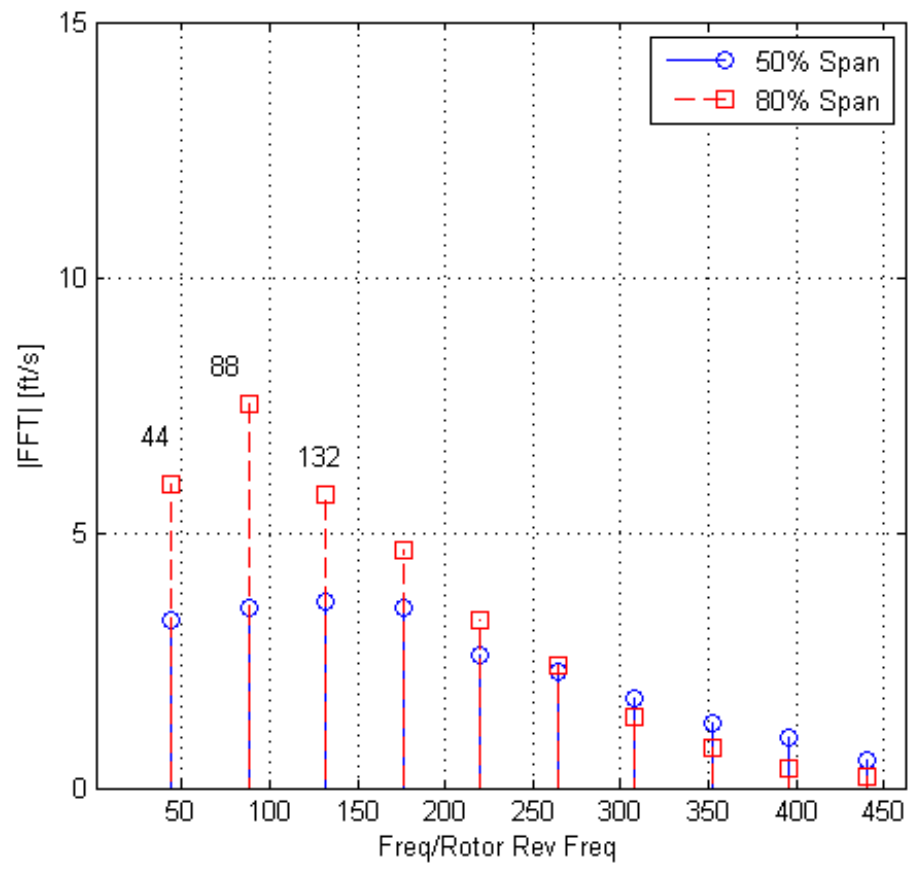

Figure 4.14: FFT of S1 wake from hotwire traverse, NL. 


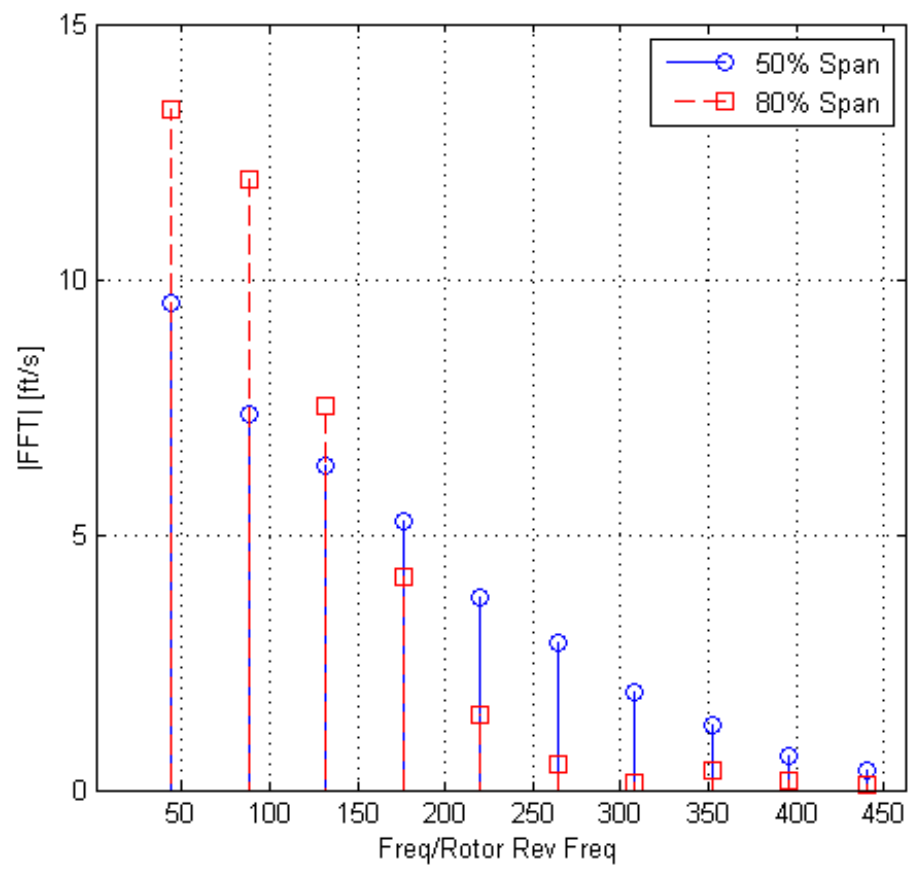

Figure 4.15: FFT of S1 wake from hotwire traverse, HL.

\subsubsection{Potential Forcing Function}

The upstream propagating potential field from Stator 2 is an additional forcing function to R2. The static pressure as measured by casing static taps is shown for both loading conditions at $74 \%$ corrected speed in Figure 4.16. The measurements were acquired across one vane pitch. In the vicinity of the S2 leading, the static pressure increases (around 90\%vp). The change in pressure associated with the potential field downstream of Rotor 2 is less than 0.1 psi. The axial location of the measurement is half way between the trailing edge of R2 and the leading edge of S2. 


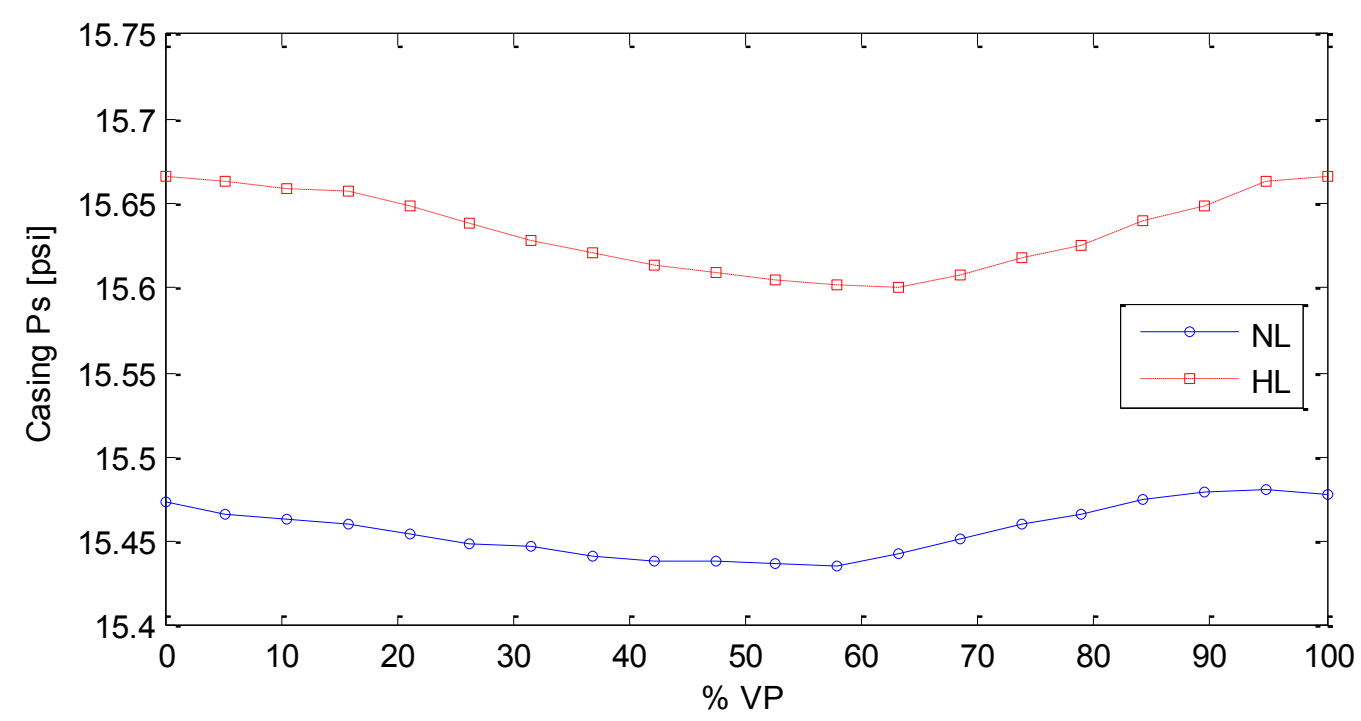

Figure 4.16: Static pressure field downstream of Rotor 2 for NL and HL.

The change in time averaged absolute flow angle due to S2 potential field is shown in Figure 4.17 and Figure 4.18. For NL at $80 \%$ span, $\alpha$ changes by approximately 3 degrees and 1.5 degrees at $50 \%$ span. This is amplified to just over 4 degrees at $80 \%$ span for HL and 2.5 degrees at $50 \%$ span. In both cases a higher alpha is in vicinity of S2 leading edge $(90 \% \mathrm{vp})$. There is an offset of approximately $25 \%$ of the vane passage in the troughs of the alpha potential field at NL that disappears at HL. 


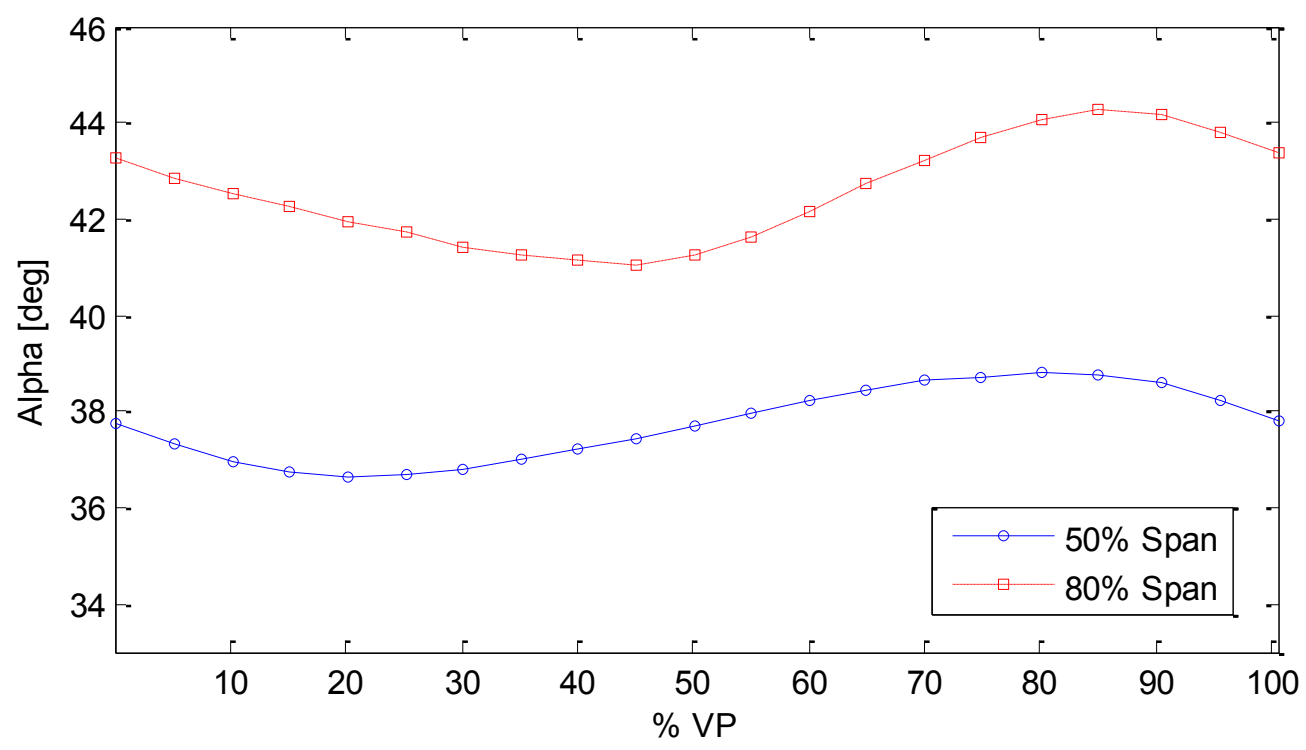

Figure 4.17: Calculated $\alpha$ from a 20 point hotwire traverse of R2 exit, NL.

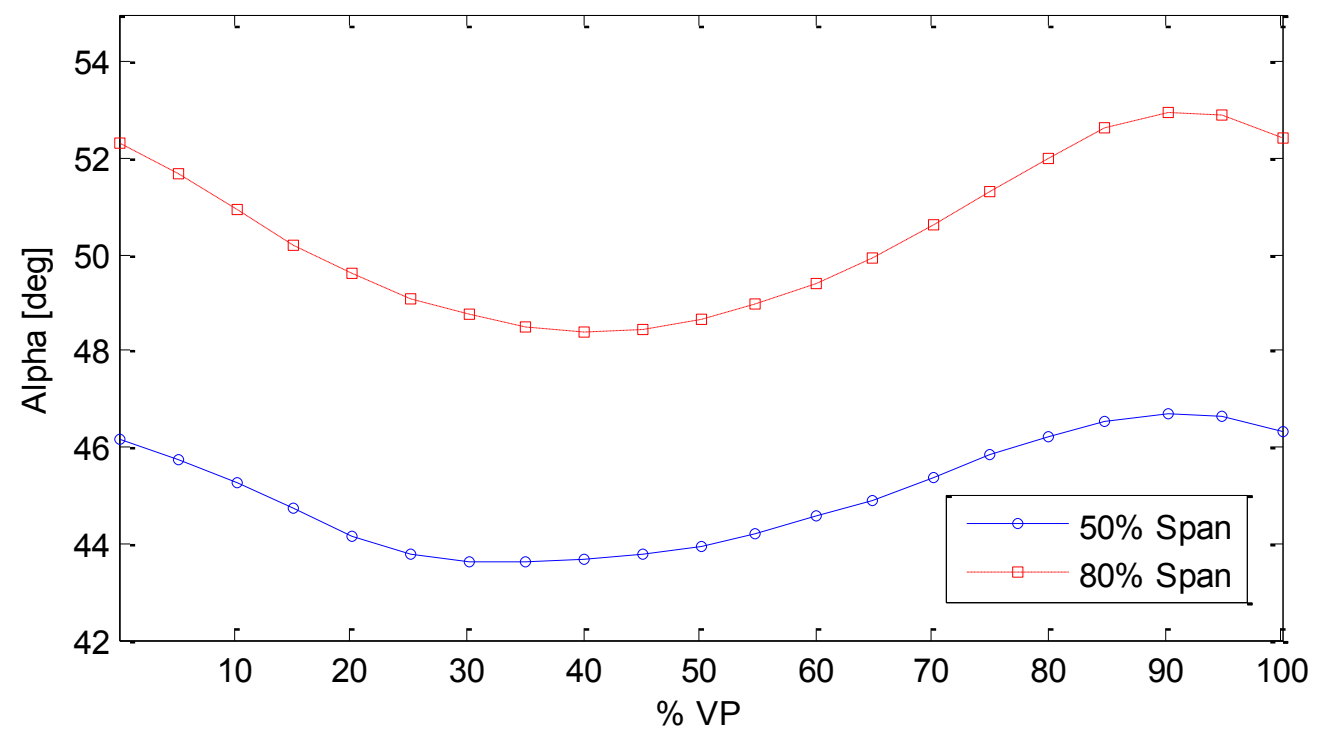

Figure 4.18: Calculated $\alpha$ from a 20 point hotwire traverse of R2 exit, HL. 


\subsection{Over-Rotor Static Pressure}

In this section the measurements from over-rotor casing-mounted Kulites will be presented. The Kulites were arranged in a $5 \times 5$ grid, and all results will be displayed in the same layout shown in Figure 4.19.

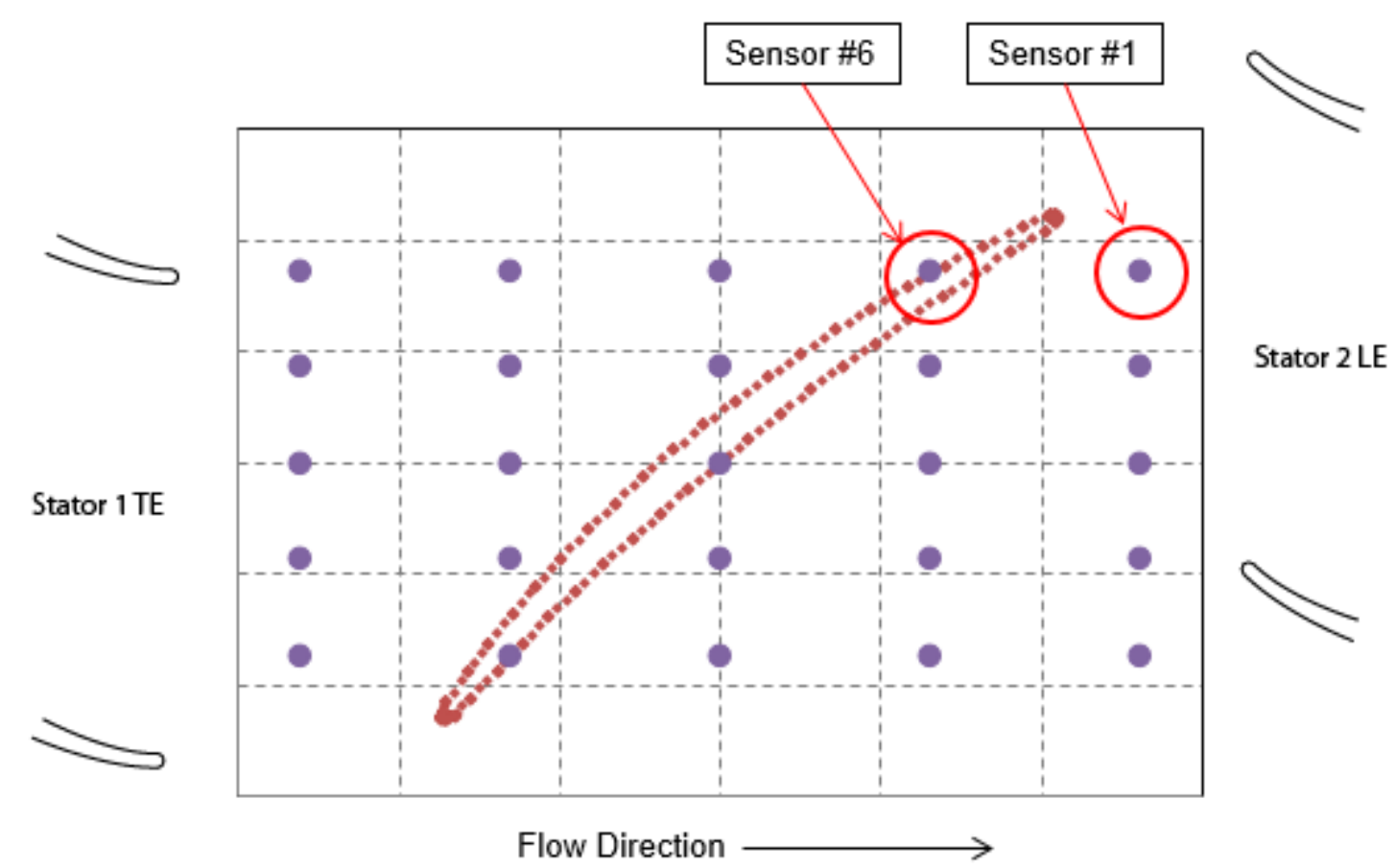

Figure 4.19: Casing Kulite arrangement over Rotor 2.

All casing Kulite pressures were time-averaged and plotted in a contour plot at steady corrected speed of 4100 RPM for 600 revolutions of data at both loading conditions in Figure 4.20. Flow direction is from left to right, and rotor rotation from top to bottom. The standard deviation associated with taking the time average of each channel provides an indication of the level of unsteadiness in this measurement. For the same loading and speed condition shown in Figure 4.20, the standard deviation is shown in Figure 4.21. The largest standard deviation in pressure occurs over the center of the blade, as would be expected 
from a higher amplitude pressure wave at the center of the blade. In these measurements, the stator vanes are at the top and bottom of the figure so that the middle of the figure, where the unsteadiness is largest, is in the same circumferential position as the mid-passage region between the two stator vane leading edges.

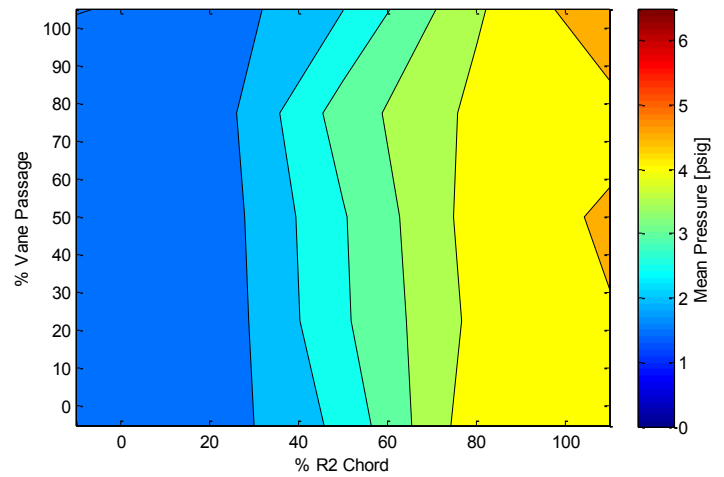

(a)

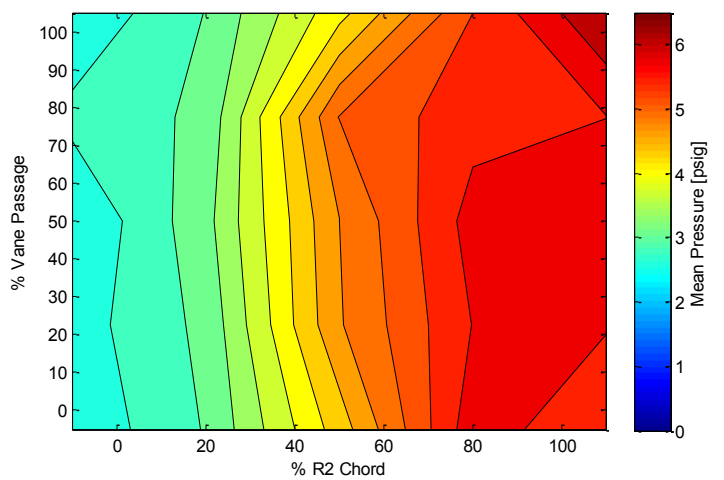

(b)

Figure 4.20: Contour plot of time averaged casing Kulite pressures at 4100 RPM, flow from left to right, rotor rotation from top to bottom. (a) NL, (b) HL.

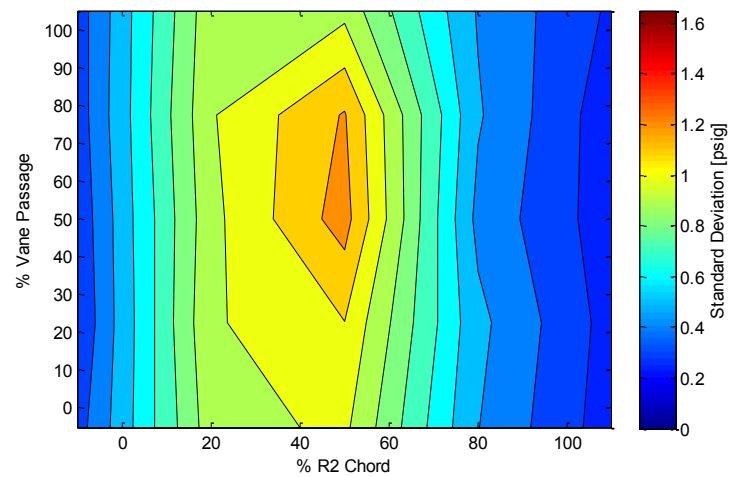

(a)

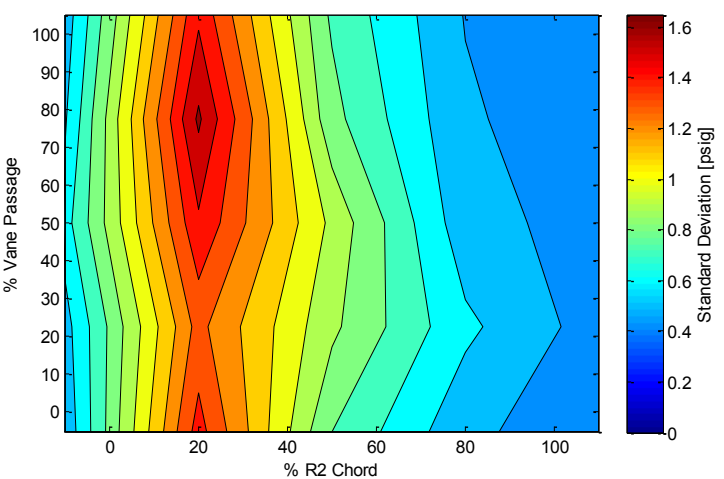

(b)

Figure 4.21: Standard deviation of mean pressure signal for casing Kulites at 4,100 RPM.

(a) NL, (b) HL. 
Figure 4.22 shows an overlay of 3 blade pass events for each of the 25 sensors, at nominal loading (blue) and high loading (red), respectively. Each subplot is shown with the same ranges as that labeled in the top left plot to allow easy comparison. It is evident that there is a gradual rise in static pressure across the rotor of approximately 2 psi. The blade pressure traces are very noticeable at the blade leading edge and mid-chord, and still noticeable at the trailing edge, although less so. The sensors both upstream and downstream of the rotor blades do not show a strong trace of $\mathrm{R} 2$ blade passage and instead seem driven by other local flows. The blade passage events were phase shifted to occur at similar locations on the $\mathrm{x}$-axis through the use of cross-correlation of each signal to the sensor in the center of the plot (column 3, row 3). 

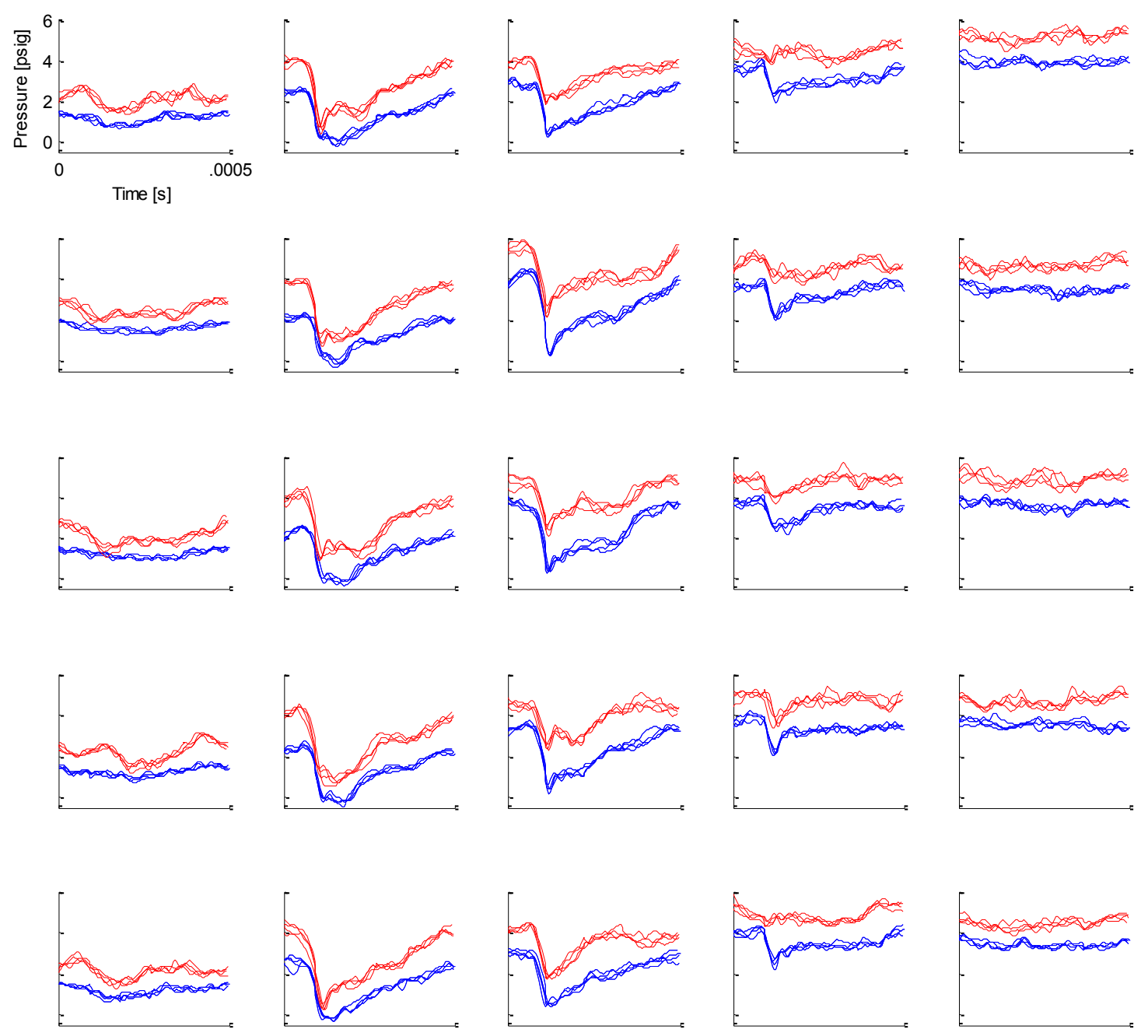

Figure 4.22: Overlaid selected pressure traces from casing Kulites, NL blue, HL red. Flow $\mathrm{L}$ to $\mathrm{R}, \mathrm{R} 2$ rotation top to bottom.

At high loading in Figure 4.22 the pressure signal at the trailing edge and downstream of the blade is much noisier than during NL. At HL, the pressure change across the blade is larger and sensors upstream of the row show influence of the blade passage events. There are no strong trends in the pressure trace as a function of circumferential location, meaning that the forcing functions do not seem to have an influence that is readily apparent through visual inspection of the pressure signal. 
The spectrum of these signals is analyzed by taking the FT of 500 revolutions of the steady data. Figure 4.23 shows results at NL. The blade pass frequency (33/rev) and its harmonics are shown and dominate the spectra. Sensors placed at mid chord and at the leading edge show the largest amplitudes in the $1^{\text {st }}$ and $2^{\text {nd }}$ harmonic (33/rev and 66/rev) of rotor 2 blade pass frequency, which makes sense by viewing the strength of the blade pass signature from the time-based plots. The strength of $\mathrm{R} 2$ harmonics decreases as the harmonic number increases on all sensors. The upstream sensors seem to show a stronger influence from R2 blade pass harmonics than the downstream sensors, even though the difference is quite small. Results at HL are shown in Figure 4.24 where the strength of the harmonics increases with HL, especially at the leading edge Kulites. It is also very evident that the strength of the second order harmonic in the mid chord sensors is a strong function of circumferential location, where the sensor at approximately $50 \% \mathrm{~S} 1$ vane passage showing the largest second harmonic amplitude. Similarly, at trailing edge Kulites, the strength of the $1^{\text {st }}$ harmonic of BPF seems to follow the same trend. This may be due to the much stronger influence of stator wakes during high loading, and their effects are felt throughout the rotor 2 passage.

Figure 4.25 and Figure 4.26 show the same spectra as Figure 4.23 and Figure 4.24 but with a reduced ordinate to illustrate the relative strengths of the R1 and R3 BPF harmonics which are multiples of 36 or 30 , respectively. The strength of the R1 BPF component (36), and higher harmonics, are evident in the upstream and leading edge Kulites, and components of R3 (30) spectra and higher harmonics are evident in the trailing edge and downstream Kulites. In fact, there are some Kulites that show a stronger influence from R3 BPF spectra than R2 BPF spectra, especially at S2 mid passage. At high loading, 
in Figure 4.26, this is even more evident, with $30 /$ rev spectra dominating the lower order harmonics. There are also several multiples of each rotor blade combination evident in the second harmonics, with 69/rev and 63/rev components evident. The 3/rev and blade pass frequency $+/-2 /$ rev occur due to the interference resulting from rotor-rotor interactions because of blade counts that differ by 3 . In Figure 4.26 the sensor at the trailing edge, $2^{\text {nd }}$ row from the top, shows almost no evidence of other harmonics than the 66/rev R2 second harmonic, indicating that the non-rotor blade multiple higher order harmonics are strongly dependent on rotor blade position with respect to stator vane position. 

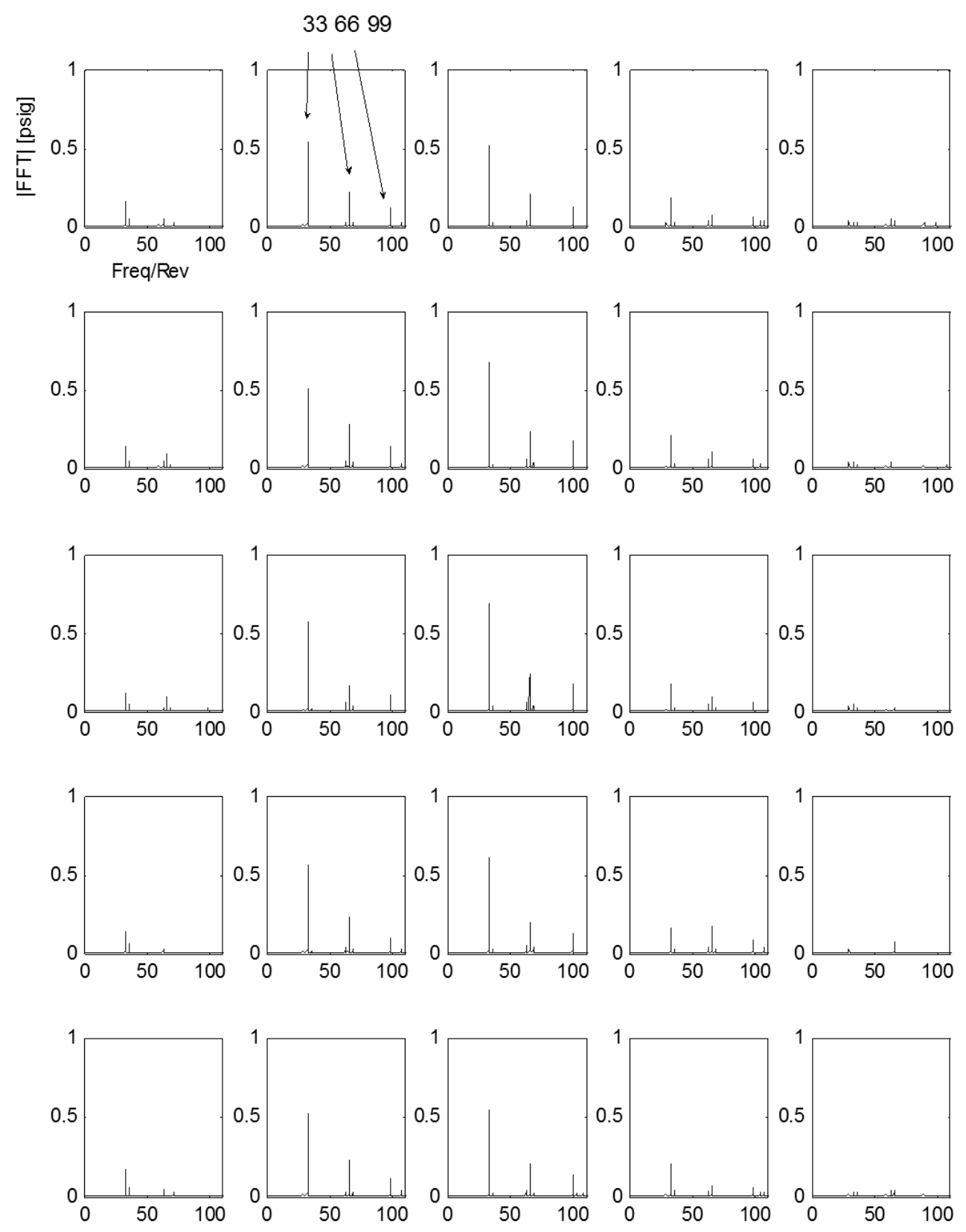

Figure 4.23: FT spectra of casing Kulites, NL, with focus on R2 BPF spectra. Flow L to $\mathrm{R}, \mathrm{R} 2$ rotation top to bottom. 

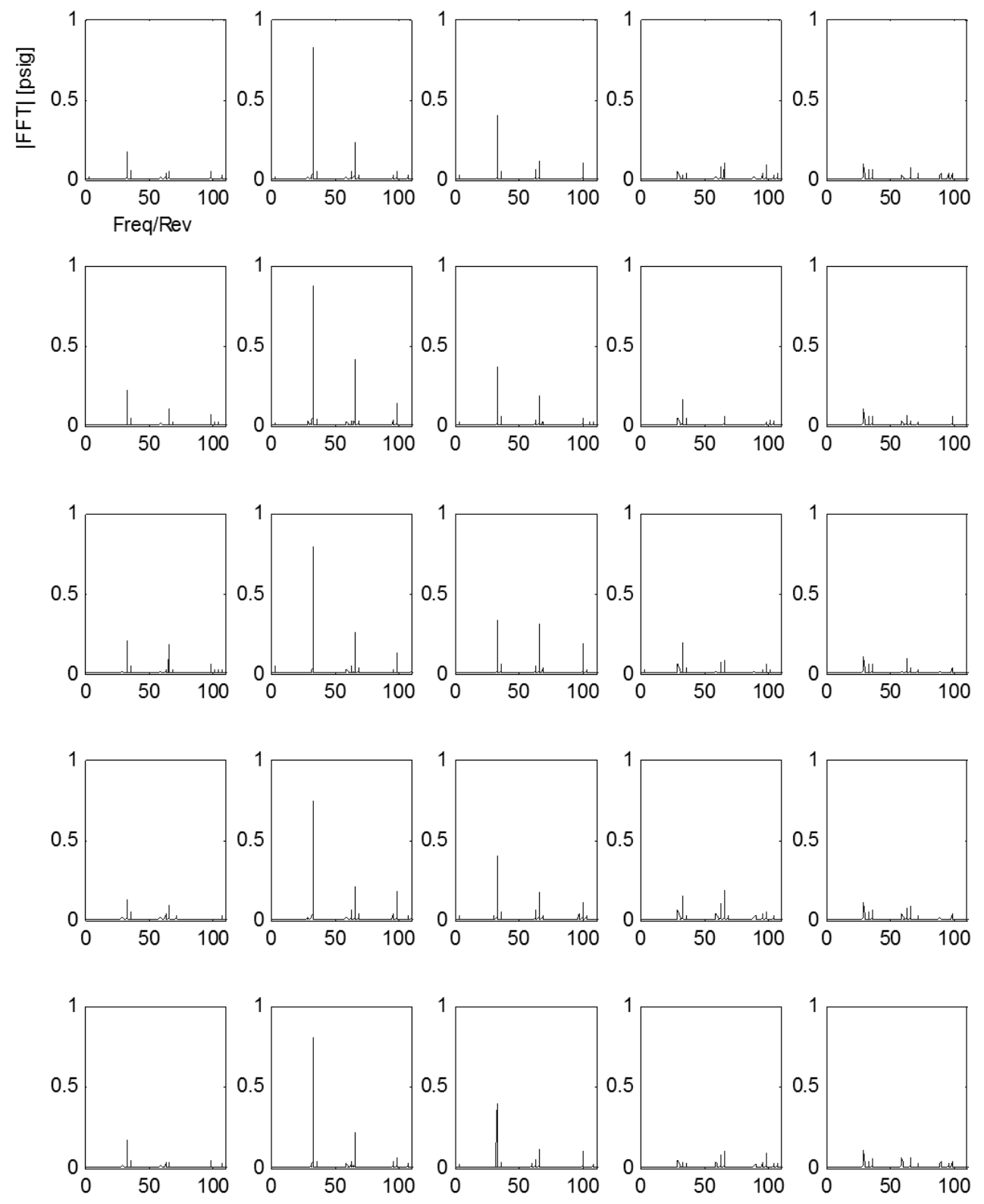

Figure 4.24: FT spectra of casing Kulites, HL, with focus on R2 BPF spectra. Flow L to $\mathrm{R}, \mathrm{R} 2$ rotation top to bottom. 

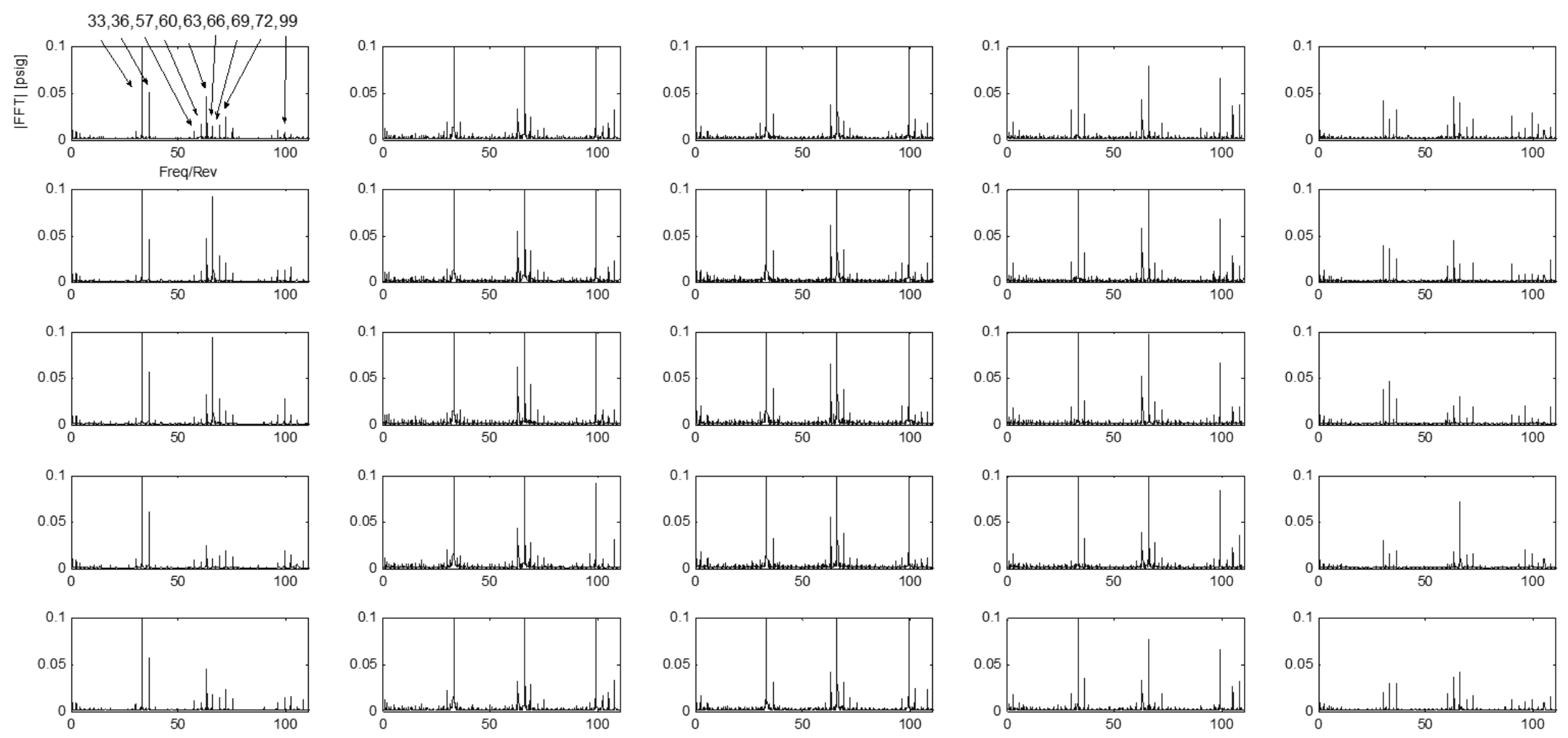

Figure 4.25: FT spectra of casing Kulites, NL, with focus on R1 and R3 BPF spectra. Flow L to R, R2 rotation top to bottom. 

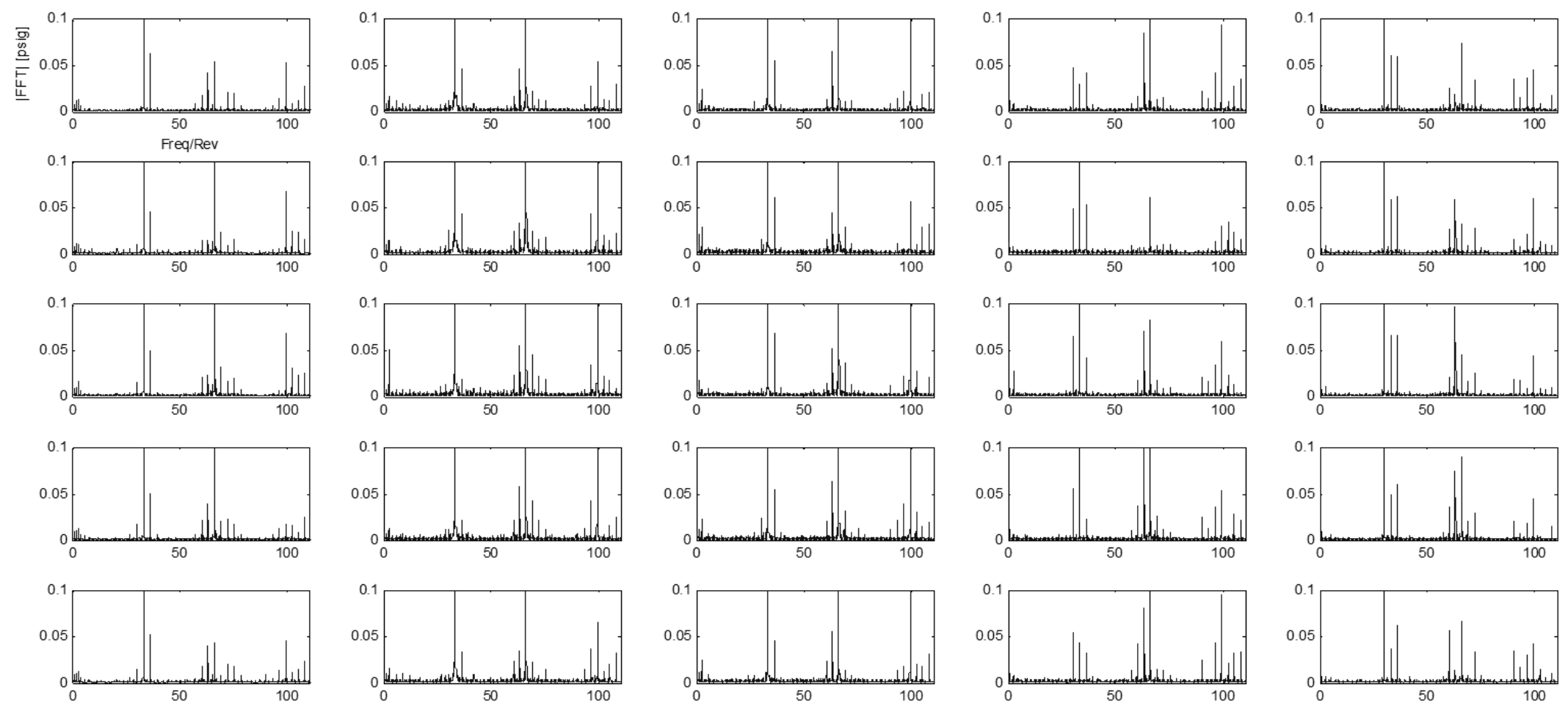

Figure 4.26: FT spectra of casing Kulites, HL, with focus on R1 and R3 BPF spectra. Flow L to R, R2 rotation top to bottom. 


\section{$4.4 \quad \underline{\text { R2 Response }}$}

Rotor response data acquired at resonance for the $1 \mathrm{~T}$ mode at the $44 \mathrm{EO}$ crossing will be presented in this section. A sample screen shot of the results from the Agilis software is shown in Figure 4.27. The red diagonal line shows the constant sweep rate of the compressor speed through the resonance. This graph shows compressor speeds from 3634 to 3801 RPM. The ordinate of the graph is the blade number, showing the results of the light probe correlation for each of the $33 \mathrm{R} 2$ blades. For each blade, the phase is shown. A 180 degree phase change is expected at resonance detection. The magnitude of the tip deflection and the compressor speed at which this occurs is recorded for each blade number. The results are summarized in the following figures.

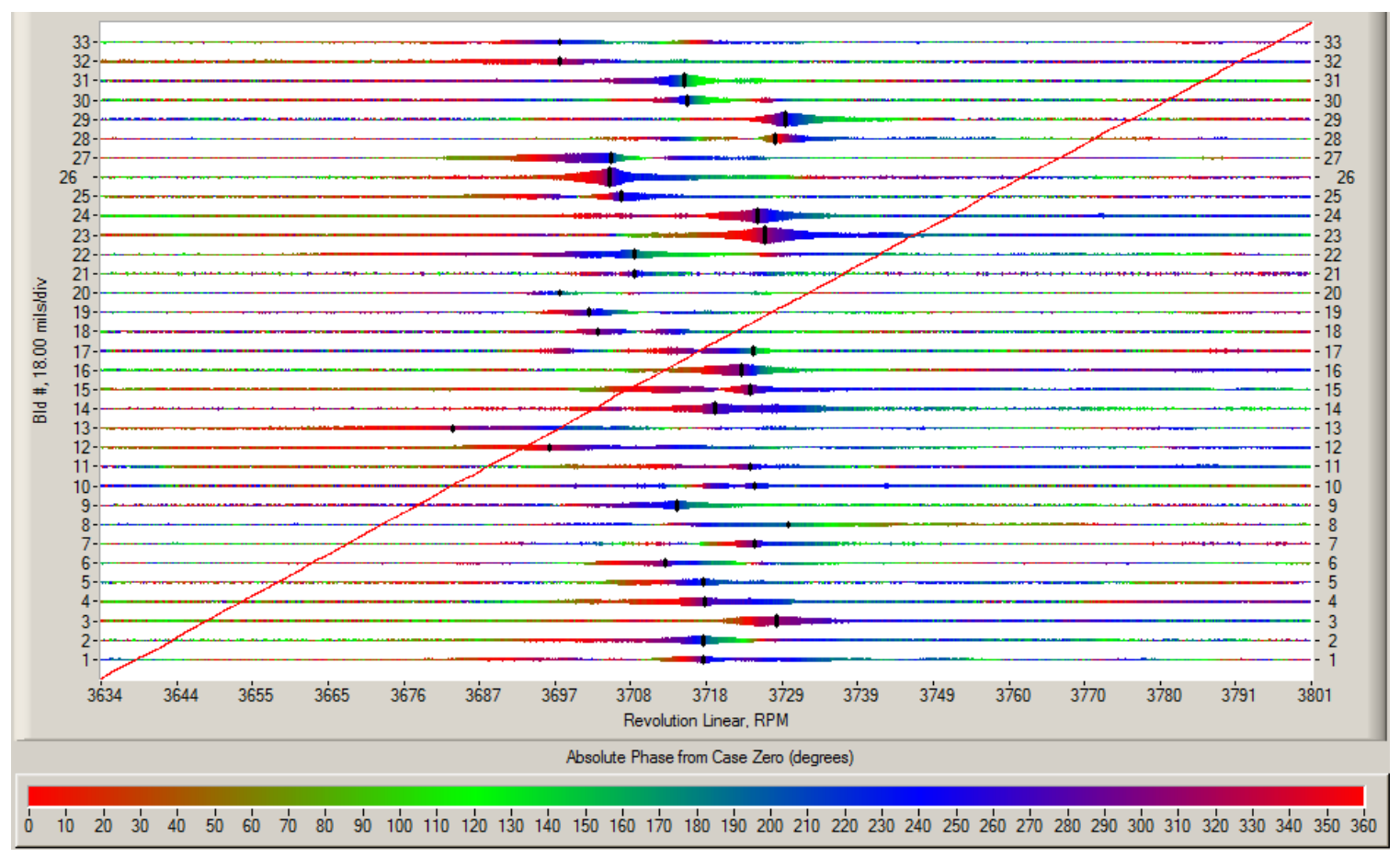

Figure 4.27: Agilis NSMS software output showing relative amplitude of blades throughout $1 \mathrm{~T}$ response, NL Accel. 
Figure 4.28 displays the maximum peak-to-peak deflection detected for each blade for four different data runs at 2 different loadings and at 2 different sweep directions, either accelerating or decelerating. The largest responding blade in all runs is blade 26 and the lowest responding blade is either blade 20 at NL or blade 13 at HL. The highest responding blade responds at an amplitude almost 4 times that of the lowest responding blade. The uncertainties of blade response vary blade-to-blade, ranging from a maximum of $+/-1.998$ mil on NL deceleration, to a max of +/- 3.078 mil on HL deceleration.

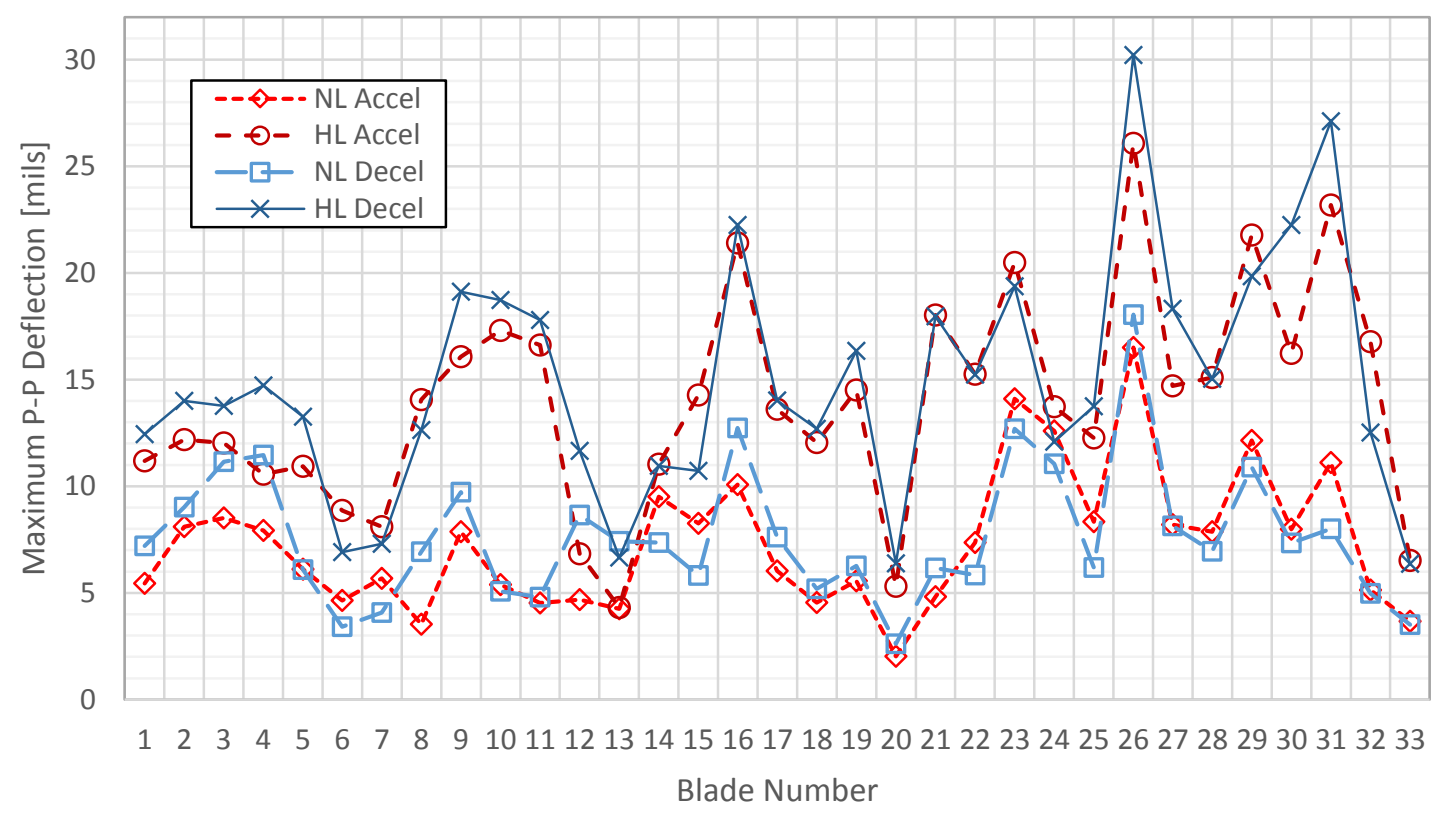

Figure 4.28: Rotor 2 maximum peak-to-peak deflection per blade.

The largest difference in runs occurs between Nominal and High Loading. Some blades, such as blade 21, respond up to three times as much under High Loading conditions as compared to Nominal loading, whereas blade 13 experiences virtually no change. When looking solely at High Loading, the lowest responding blade is blade 13, as compared to Nominal loading, where blade 20 was the lowest responder. The changes in loading do not, 
however, greatly affect the frequency at which the blades respond. A few blades, such as blade 12 , seem to respond at a slightly different compressor speed when the loading is changed, but almost all blades indicate relatively no change. The response amplitude of the blades as a function of frequency, Figure 4.29 and Figure 4.30, show each blade as an individual line. The large difference in amplitude response due to loading is apparent, and there is evidence of multiple "peaks" in the responses of some blades. This is in part due to the blisk construction of the rotor, where excited blades can excite neighboring nonresponding blades through stresses in the blisk.

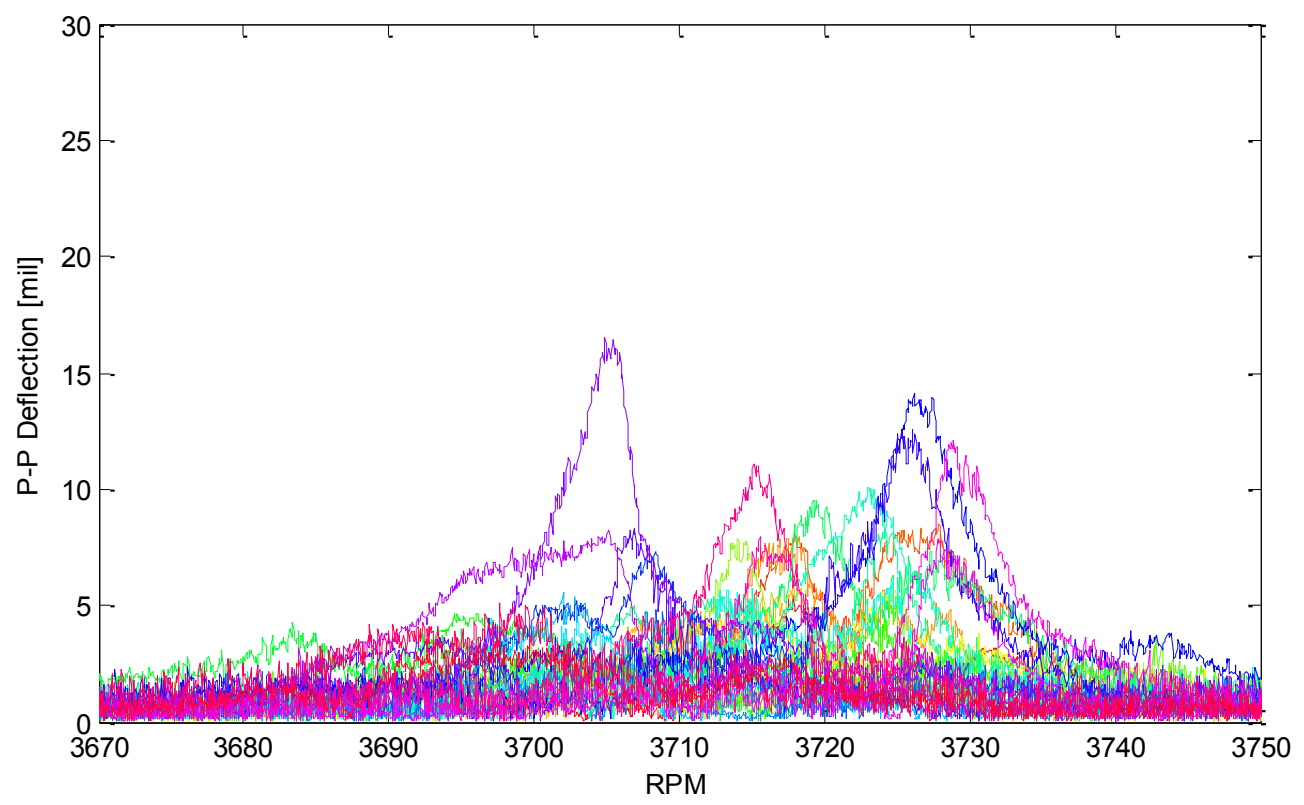

Figure 4.29: P-P response of each R2 blade at NL, overlaid. 


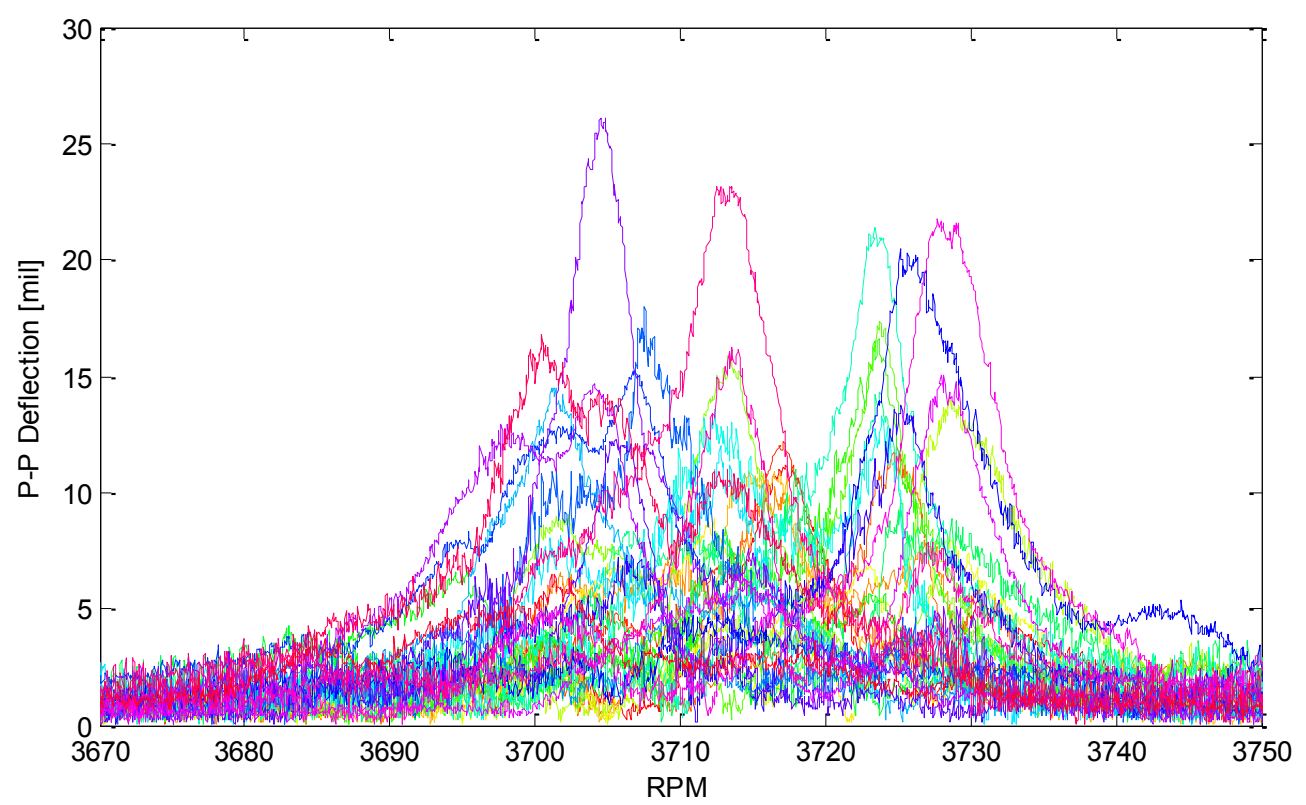

Figure 4.30: P-P response of each R2 blade at HL, overlaid.

Figure 4.31 shows how the frequency, and thus, compressor speed, at which maximum P-P response for each blade occurs and how it changes based on loading and sweep direction. Large changes are only shown in only a couple blades. 


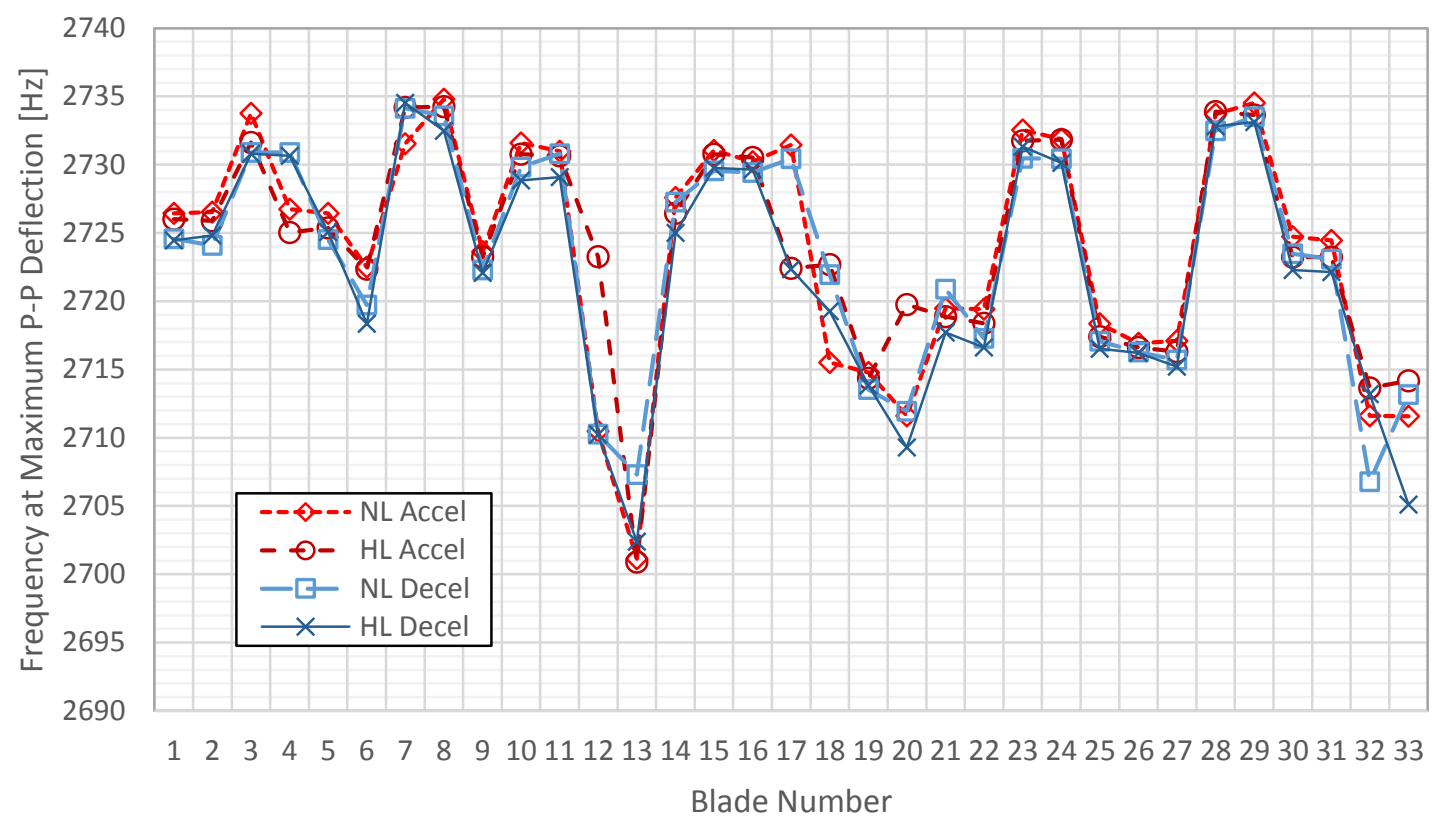

Figure 4.31: Rotor 2 frequency at point of maximum p-to-p deflection per blade.

The damping ratio calculated using a half power method for the blades is also shown as a function of blade number in Figure 4.32. The damping ratio per blade is usually greater under high loading as compared to nominal loading, indicating to some degree the effect that aerodynamic damping has on the rotor-blisk system. However, the damping ratios for the blades given by NSMS data have, in general, very small amplitudes, some of which approach zero. The Agilis Least-Squares Model Fitting (LSMF) module used to calculate the damping ratio for the blades had a nominal cutoff setting for a damping ratio less than $0.05 \%$, indicating that the magnitude of the damping, even with aerodynamic effects, is quite small. Damping is calculated by assuming a single degree of freedom massspring damper system for each individual blade, and performing a non-linear least square curve fit to resonant response data. Amplitude, resonant frequency, damping, and phase 
are iterated until a best fit is found. The software is therefore unable to differentiate between mechanical and aerodynamic damping, and thus, the damping coefficient is calculated from a combination of the two. Some of the most strongly damped blades (for example, 12, 22 and 27) tend to have average or below average vibrational responses, but they are not necessarily the lowest responding blades. This may be due to the coupled nature of the blisk system, where a greater damped blade may have an effect on the blades near it but not necessarily itself.

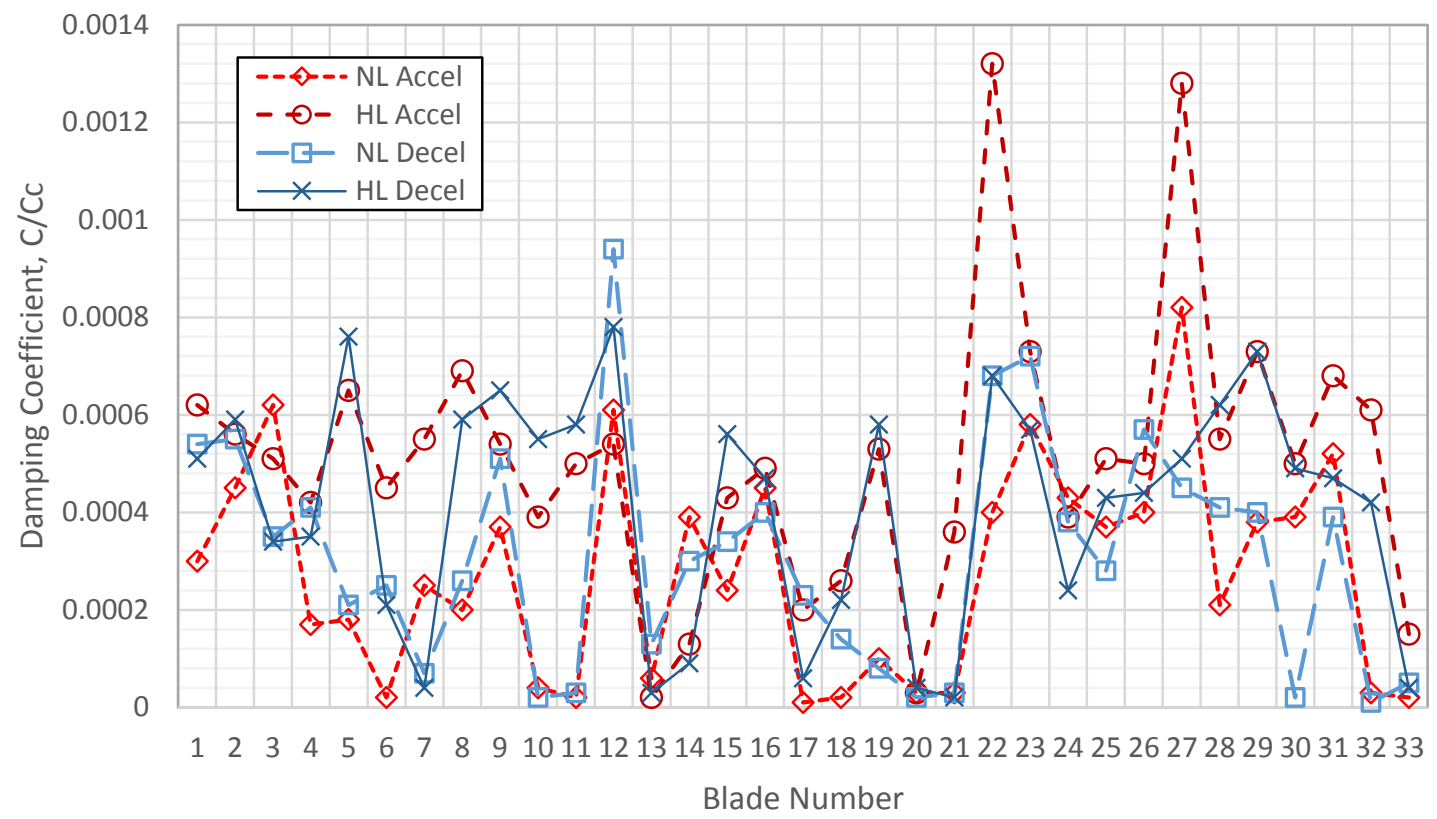

Figure 4.32: Rotor 2 damping coefficient, $\mathrm{C} / \mathrm{Cc}$, per blade.

Table 4-1 shows a summary of the minimum, maximum, and average of the presented blade response parameters. The largest differences occur between NL and HL for P-P amplitude and blade damping. Average P-P amplitude is increased with loading by $82 \%$ for acceleration cases, and $95 \%$ for deceleration. The maximum blade response out 
of all 33 blades is increased by up to $67 \%$ with loading. The average damping coefficient increases with loading by $65 \%$ for acceleration and $34 \%$ for deceleration. Frequency at which the blades responds shows a negligible change between loadings and sweep direction. 
Table 4-1: Summary of R2 blade responses through 4 different runs at 2 loadings.

\begin{tabular}{|l|l|l|l|l|l|l|l|l|l|}
\hline & \multicolumn{7}{|c|}{ P-P Amplitude [mil] } & \multicolumn{7}{l|}{ Freq. at max Amplitude [Hz] } & \multicolumn{3}{|c|}{ Damping Coefficient, C/Cc } \\
& Min & Max & Avg & Min & Max & Avg & Min & Max & Avg \\
\hline NL Accel & 2.03 & 16.51 & 7.35 & 2701.2 & 2734.8 & 2723.8 & $1.0 \mathrm{E}-05$ & $8.2 \mathrm{E}-04$ & $2.8 \mathrm{E}-04$ \\
\hline NL Decel & 2.61 & 18.05 & 7.65 & 2706.8 & 2734.1 & 2723.3 & $1.0 \mathrm{E}-05$ & $9.4 \mathrm{E}-04$ & $3.1 \mathrm{E}-04$ \\
\hline HL Accel & 4.31 & 26.07 & 13.98 & 2700.9 & 2734.2 & 2724.0 & $2.0 \mathrm{E}-05$ & $1.3 \mathrm{E}-03$ & $5.1 \mathrm{E}-04$ \\
\hline HL Decel & 6.37 & 30.21 & 14.92 & 2702.4 & 2734.5 & 2722.3 & $2.0 \mathrm{E}-05$ & $7.8 \mathrm{E}-04$ & $4.1 \mathrm{E}-04$ \\
\hline
\end{tabular}


Blisk nodal diameter data from NSMS is shown for nominal loading and high loading in Figure 4.33 and Figure 4.34 respectively. Both conditions show the highest responding ND to be 11 , which is what would be predicted from the difference between the number of forcing blades (Stator 1, with 44 blades) and the excited blade row (Rotor 2, with 33 blades). However, ND 9 to 15 also seem to show a significant response, especially for NL, and there is a difference between the response for NL and HL, where more ND response is focused in ND above 11 for the NL case.

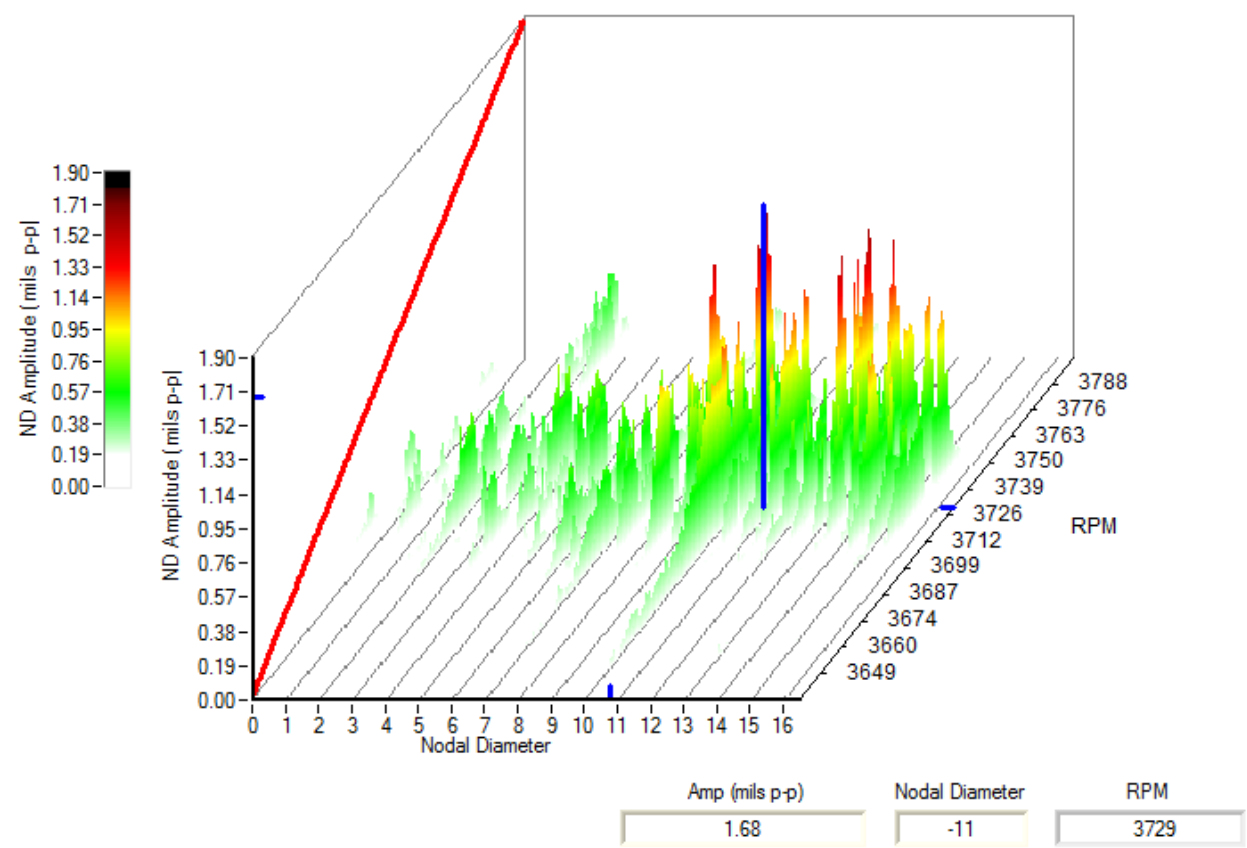

Figure 4.33: ND response from LSMF analysis on Agilis software for NL. 


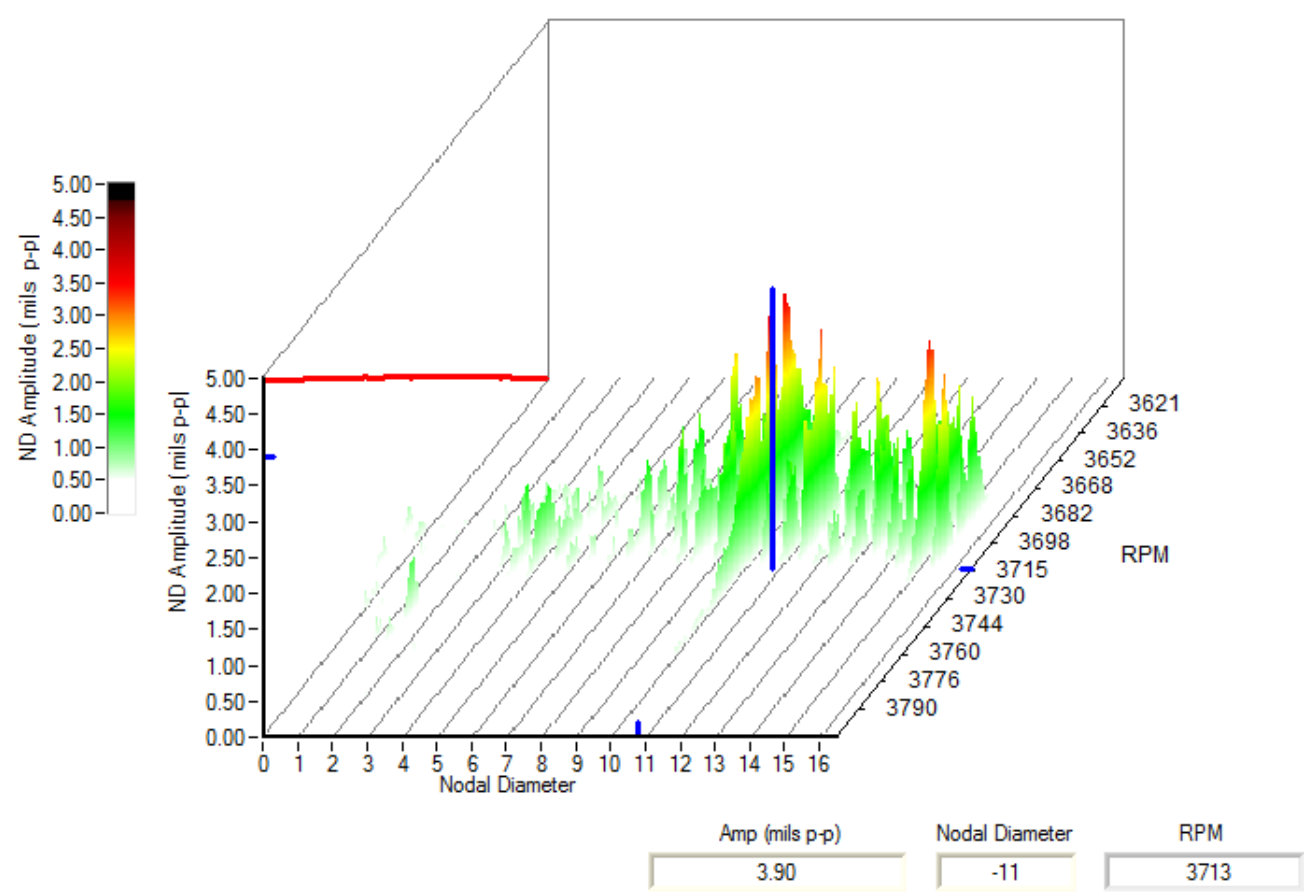

Figure 4.34: ND response from LSMF analysis on Agilis software for HL.

\section{$4.5 \underline{\text { R2-S2 Interaction }}$}

Due to limitations in budget, it was prohibitively expensive to consider the development of rotating instrumentation. Therefore, stator 2 was instrumented with 16 pressure transducers spread over locations at 50\% and 80\% span and on the Pressure Side (PS) and Suction Side (SS) of the vane row. Rotor 2 wakes were captured through the use of thermal anemometry, and a 500 revolution EA average of calculated values for absolute flow angle, alpha, are shown. This data was used to quantify the effects of R2 wakes on the unsteady lift measured by stator-mounted Kulites. 


\subsubsection{Rotor 2 Wakes}

Rotor wakes cause a velocity deficit in the flow that results in large changes in absolute flow angle, and thus, loading, on surrounding stator rows. This creates a timechanging incidence on stator vanes that result in unsteady lift forces. The potential field from the rotor blades causes a change in exit flow angle on upstream stator rows. R2 wakes at 4 different positions are shown in this section. Figure 4.35 and Figure 4.36 show absolute flow angle at two circumferential locations downstream of stator 1 - Figure 4.35 is outside of the vane wake, and Figure 4.36 is within the vane wake. Each figure displays an ensemble average of approximately 500 revolutions of calculated absolute flow angle values and a corresponding FT of the signal. The ensemble average was created by calculating absolute flow angle for the entire $\sim 500$ revolution hotwire signal, then taking the EA of the resultant signal. The FT was generated not using the ensemble average, but with the entire $\sim 500$ revolutions, in order to gain a considerably higher resolution in the frequency spectrum. Each figure shows a plot for $50 \%$ span and $80 \%$ span, at NL and HL. Figure 4.43 and Figure 4.44 show hotwire data taken at R2 exit, displaying calculated absolute flow angle or rotor wakes, and corresponding spectra. 

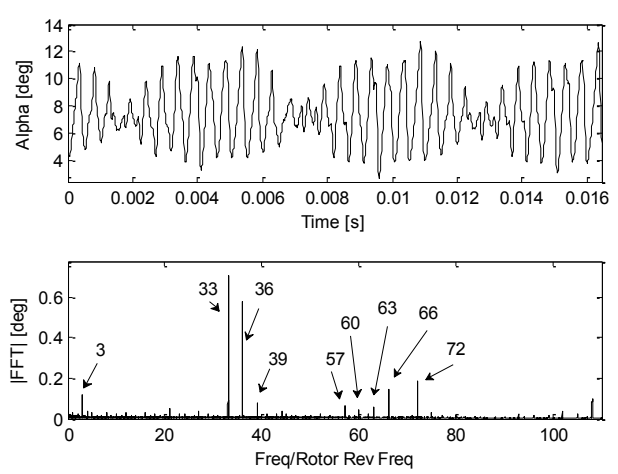

(a)
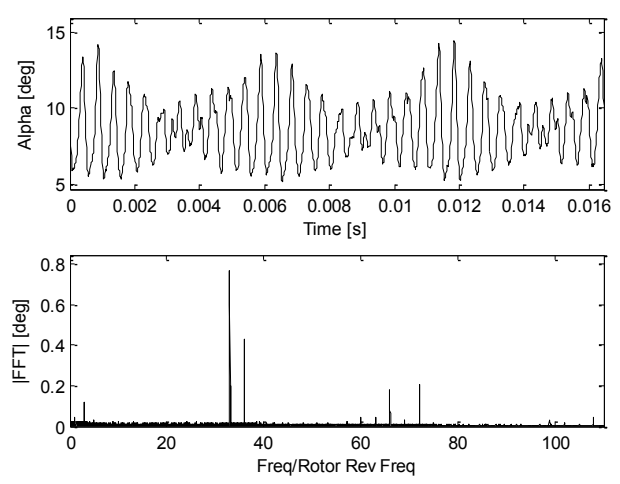

(c)
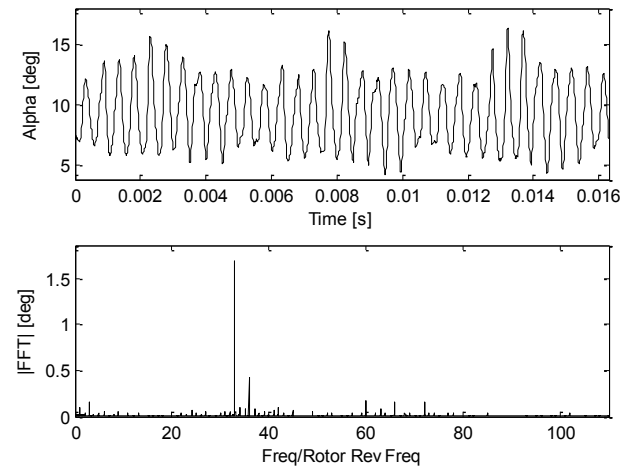

(b)
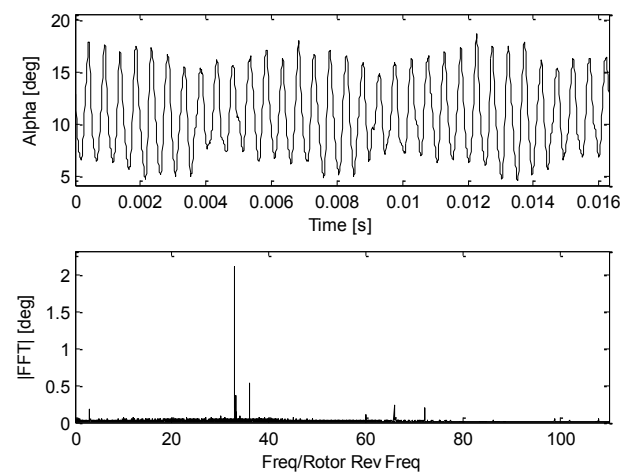

(d)

Figure 4.35: S1 Exit EA of Alpha and FFT of EA, 10\% VP. (a) 50\% Span, NL, (b) 50\% Span, HL, (c) $80 \%$ Span, NL, (d) $80 \%$ Span, HL.

Figure 4.35 shows the change in alpha from R2 blade passage outside of S1 wake. The 3-beat interference pattern is much stronger at NL than HL, and this is evident in the frequency spectra. At HL, the 33/rev component of the spectrum is much higher than all other components, and there is a weaker $3 /$ rev component suggesting less impact from rotor-rotor interference. All spectral peaks at $80 \%$ span are larger than at 50\% span. 

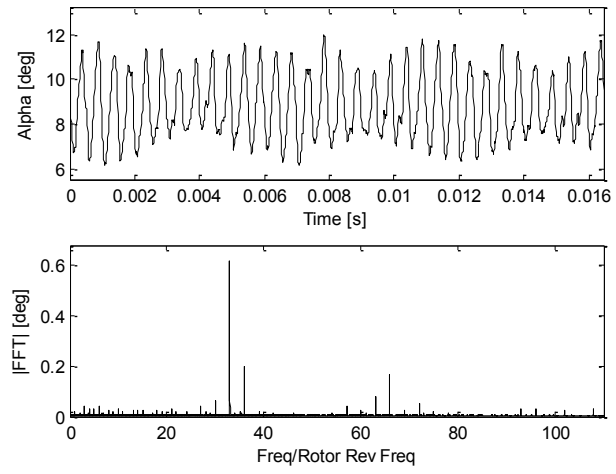

(a)
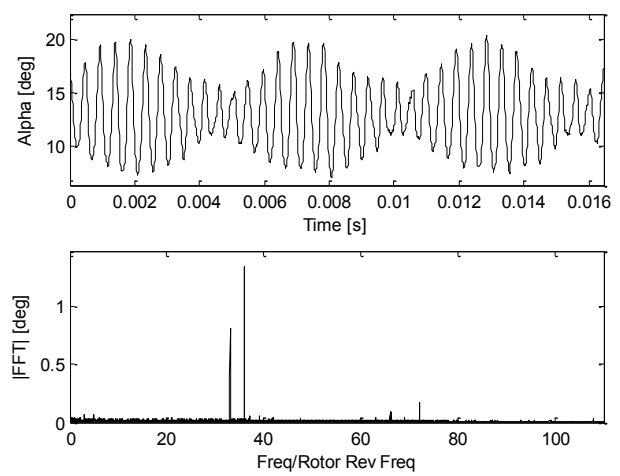

(c)
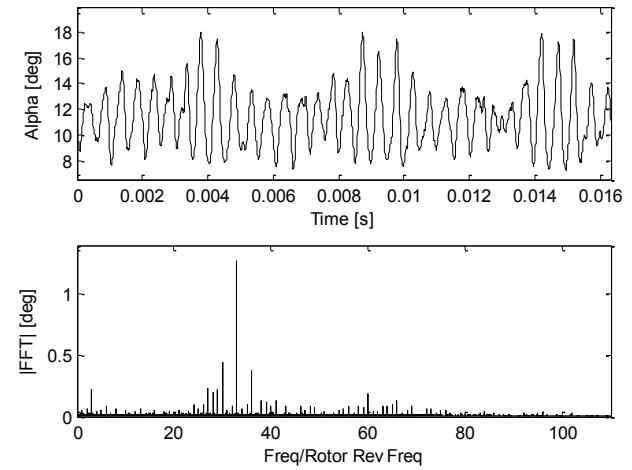

(b)
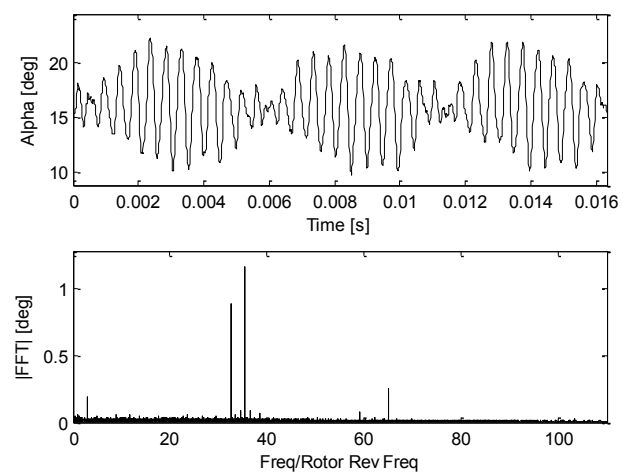

(d)

Figure 4.36: S1 Exit EA of Alpha and FFT of EA, 55\% VP. (a) 50\% Span, NL, (b) 50\% Span, HL, (c) $80 \%$ Span, NL, (d) $80 \%$ Span, HL.

Figure 4.36, within S1 wake, shows the only plots where the spectral component of $\mathrm{S} 1$ is stronger in the first harmonic than $\mathrm{R} 2$ at $80 \%$ span, which has not been replicated by any other fast-response transducer in these studies, and the 3-beat pattern is characteristically strong, even at high loading at $80 \%$ span. 

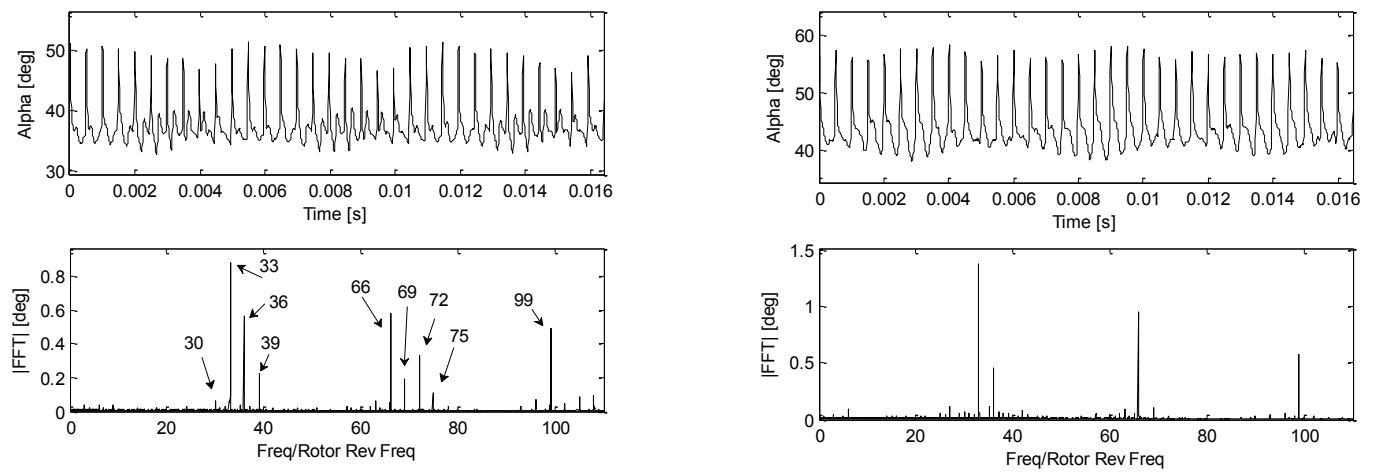

(a)

(b)
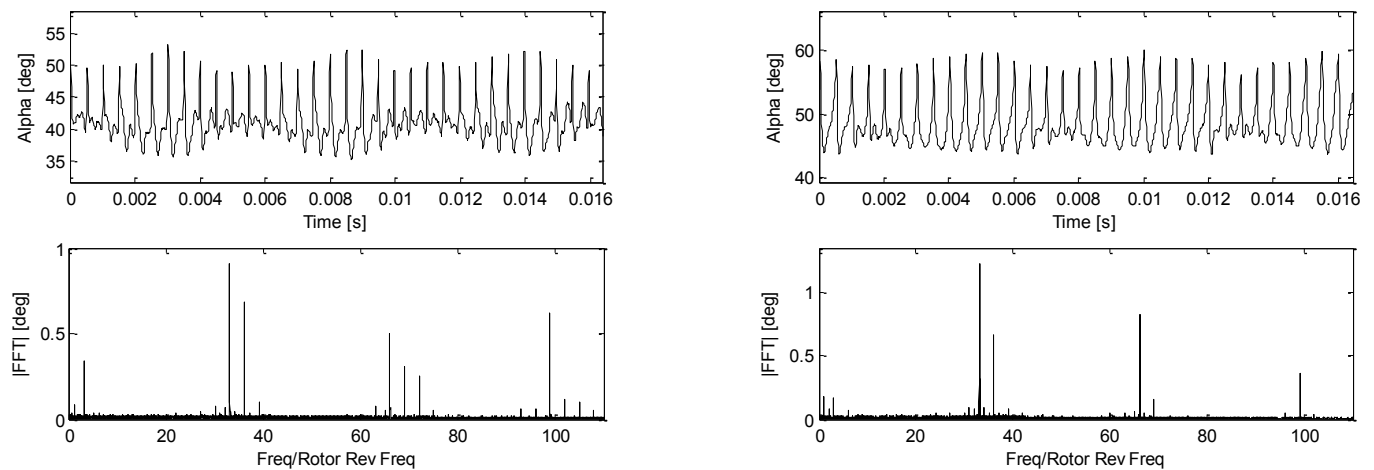

(c)

(d)

Figure 4.37: R2 Exit EA of Alpha and FFT of EA, 45\% VP. (a) 50\% Span, NL, (b) 50\% Span, HL, (c) $80 \%$ Span, NL, (d) $80 \%$ Span, HL.

Figure 4.37 shows that the characteristics of rotor wakes are strongly dependent on circumferential position. At 45\% S2 vane passage, there are strong influences from R1 (36/rev, 72/rev), and the trends in loading are similar to S1 exit hotwire data - the R2 BPF grows significantly over NL plots. At NL, $2^{\text {nd }}$ order harmonics are visible from all rotors, but these tend to be drowned out at high loading. At $80 \%$ span, the wakes from R1 seem to have a strong influence on the EA alpha signature, effectively raising the troughs significantly in-between each R2 blade passage event. 

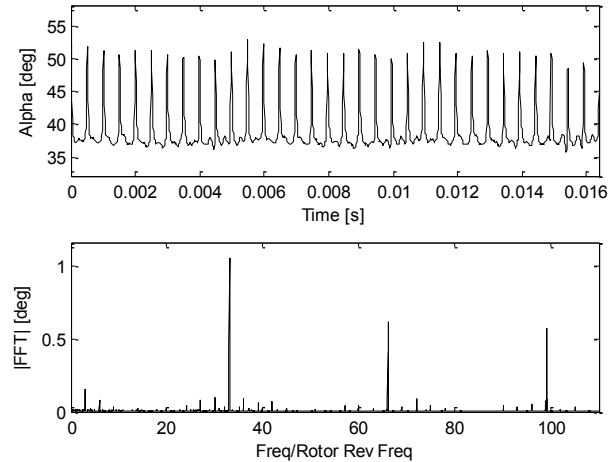

(a)
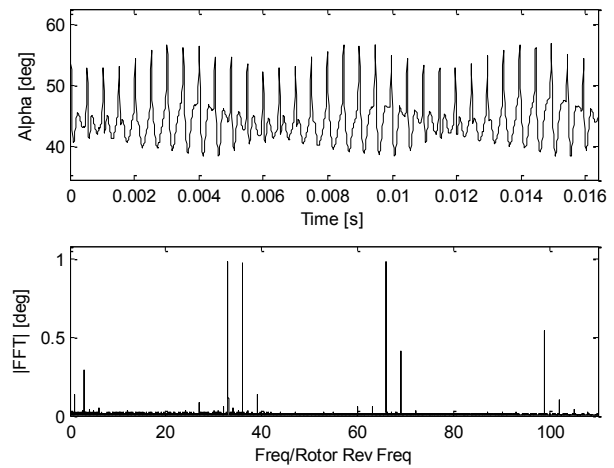

(c)
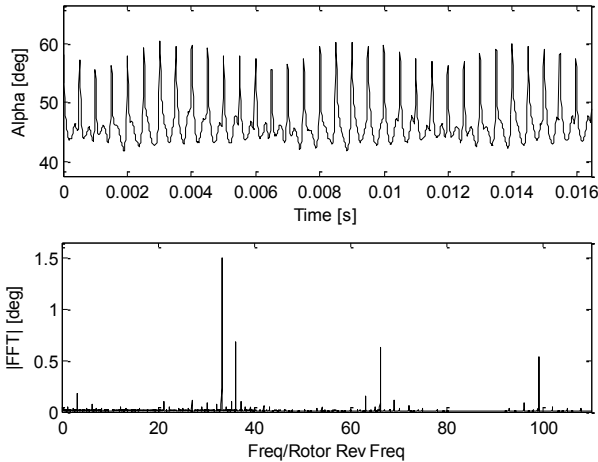

(b)
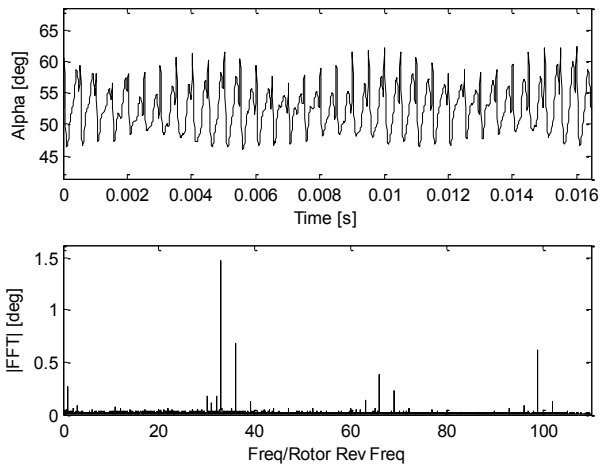

(d)

Figure 4.38: R2 Exit EA of Alpha and FFT of EA, 85\% VP. (a) 50\% Span, NL, (b) 50\% Span, HL, (c) $80 \%$ Span, NL, (d) $80 \%$ Span, HL.

Figure 4.38 shows a large change in the spectra and signal between $50 \%$ and $80 \%$ span. At NL 50\% span, there is virtually no evidence of multistage effects. However, at a higher span location, the influence from R1 in the FT is almost as strong in the $1^{\text {st }}$ harmonic as R2, and it is visually evident in the interference pattern and 3-beat pulsation shown by the waveform. At high loading, the plots become more similar, and R1 influences are not as strong. However, $2^{\text {nd }}$ harmonic spectra from R1 are not as strong as R2, even when $1^{\text {st }}$ harmonic spectra are nearly equal in amplitude, shown by the $80 \%$ span location at NL. 
This is strong evidence for the fact that higher-order frequency spectra decay more quickly with distance and are harder to measure at long distances. Average rotor 2 alpha variability is about 16 degrees, and increases slightly with increased loading.

\subsubsection{Stator 2 Loading}

Since the number of sensors limits the resolution of computed unsteady lift, unsteady surface pressure measurements will instead be shown. Figure 4.39 shows the blade loading averaged throughout 500 revolutions of data on the pressure and suction side of the stator section, at both $50 \%$ and $80 \%$ span locations. The sensor at Pressure Side, 50\% Span, 20\% Chord was not functioning during any of the data runs, and therefore did not provide any useful data. This Kulite was not functioning upon arrival from Kulite, where it was installed post machining.

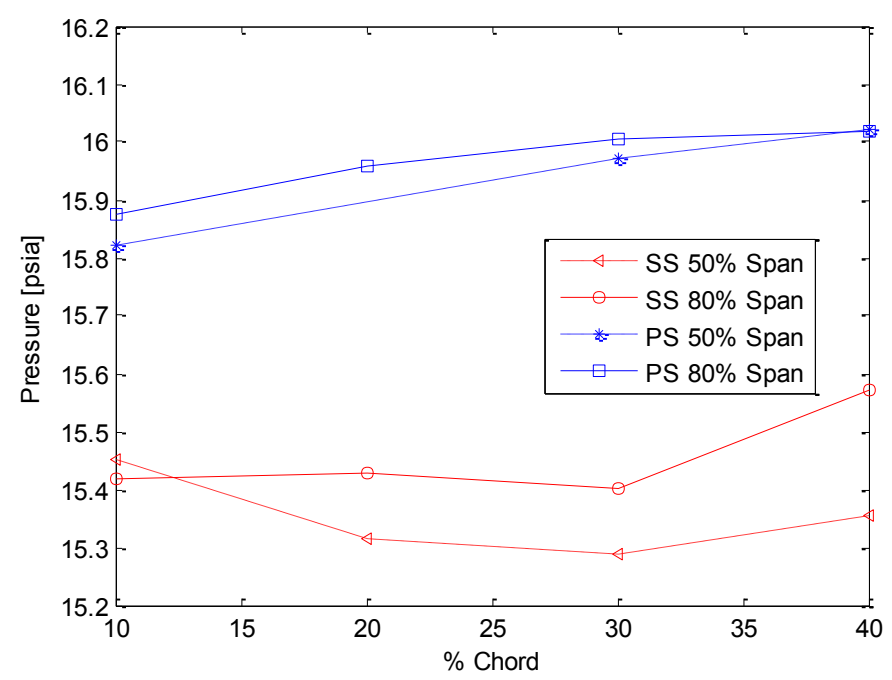

Figure 4.39: Mean pressures on SS and PS of Stator 2, NL. 


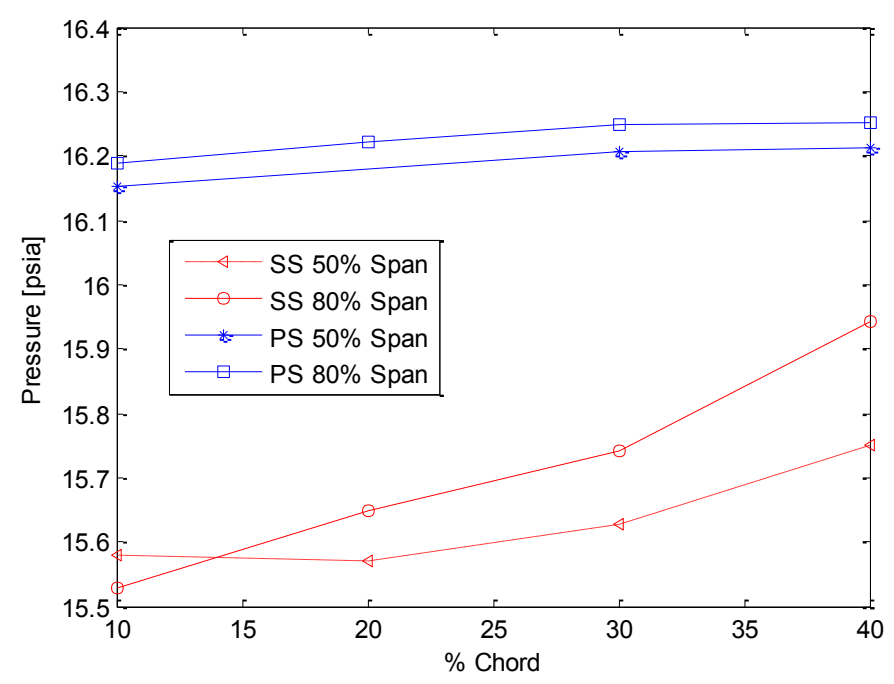

Figure 4.40: Mean pressures on SS and PS of Stator 2, HL.

Because of the nature of the unsteady data, there are a multitude of ways to display the unsteady component of blade loading. At $80 \%$ span, the same average pressure as shown in Figure 4.39 is shown for both pressure and suction side of the blade. Figure 4.40 is analogous to Figure 4.39, showing instead average pressures during HL. Figure 4.41 through Figure 4.44 show a time-varying view of the stator pressure transducers. For both span locations and loadings. By starting at the top left image and moving in a clockwise progression, the plots show how the blade loading changes as a blade on rotor 2 traverses the S2 vane passage. For 9 subplots, the percent that R2 advances across the vane passage is divided into 9 increments, each 12.5 degrees VP apart from each other. The dotted line shows the time-average pressure for each of the transducers over the entire run through resonance. 

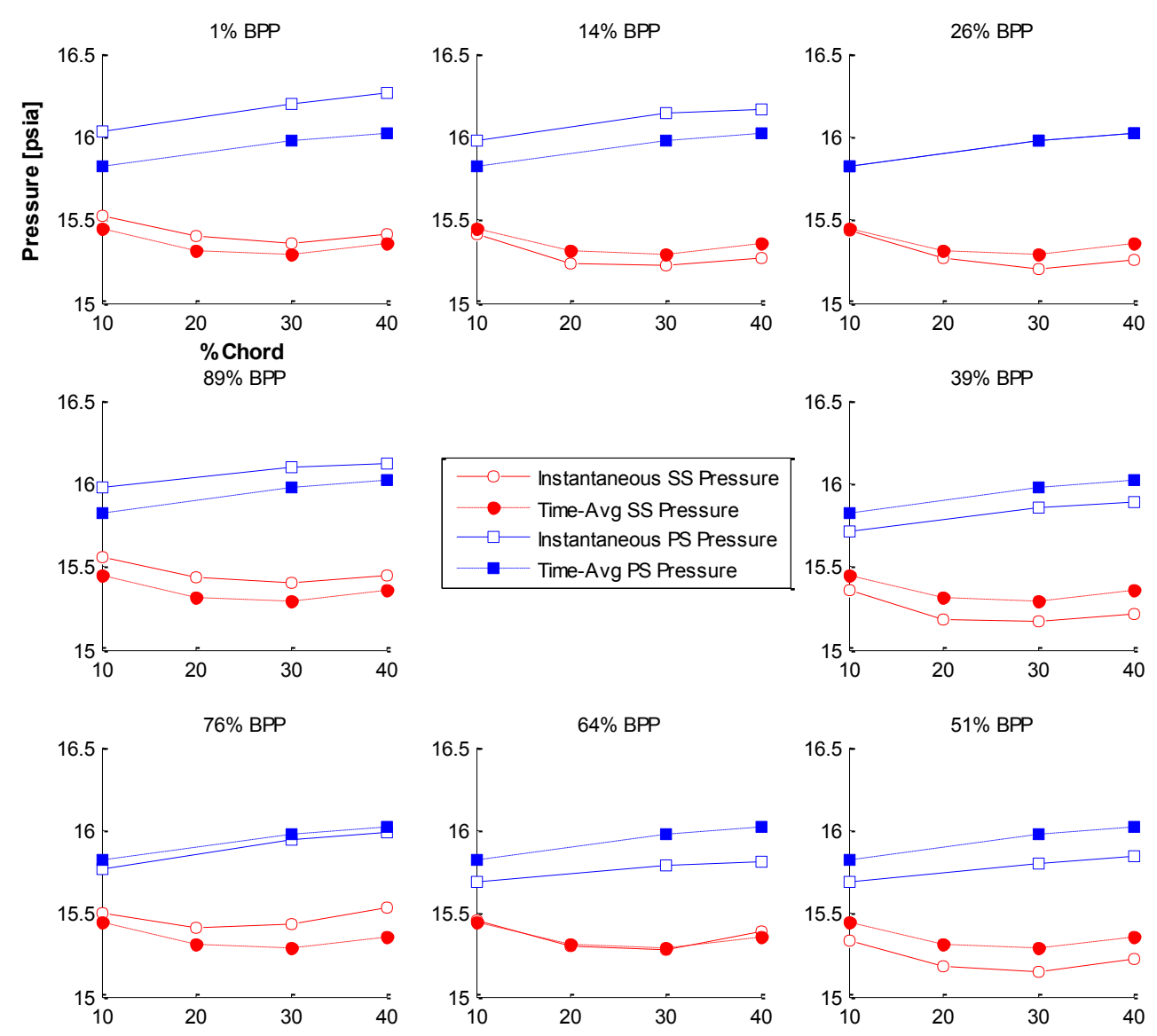

Figure 4.41: Stator Kulite blade loading as a function of rotor position, 50\% Span NL. 

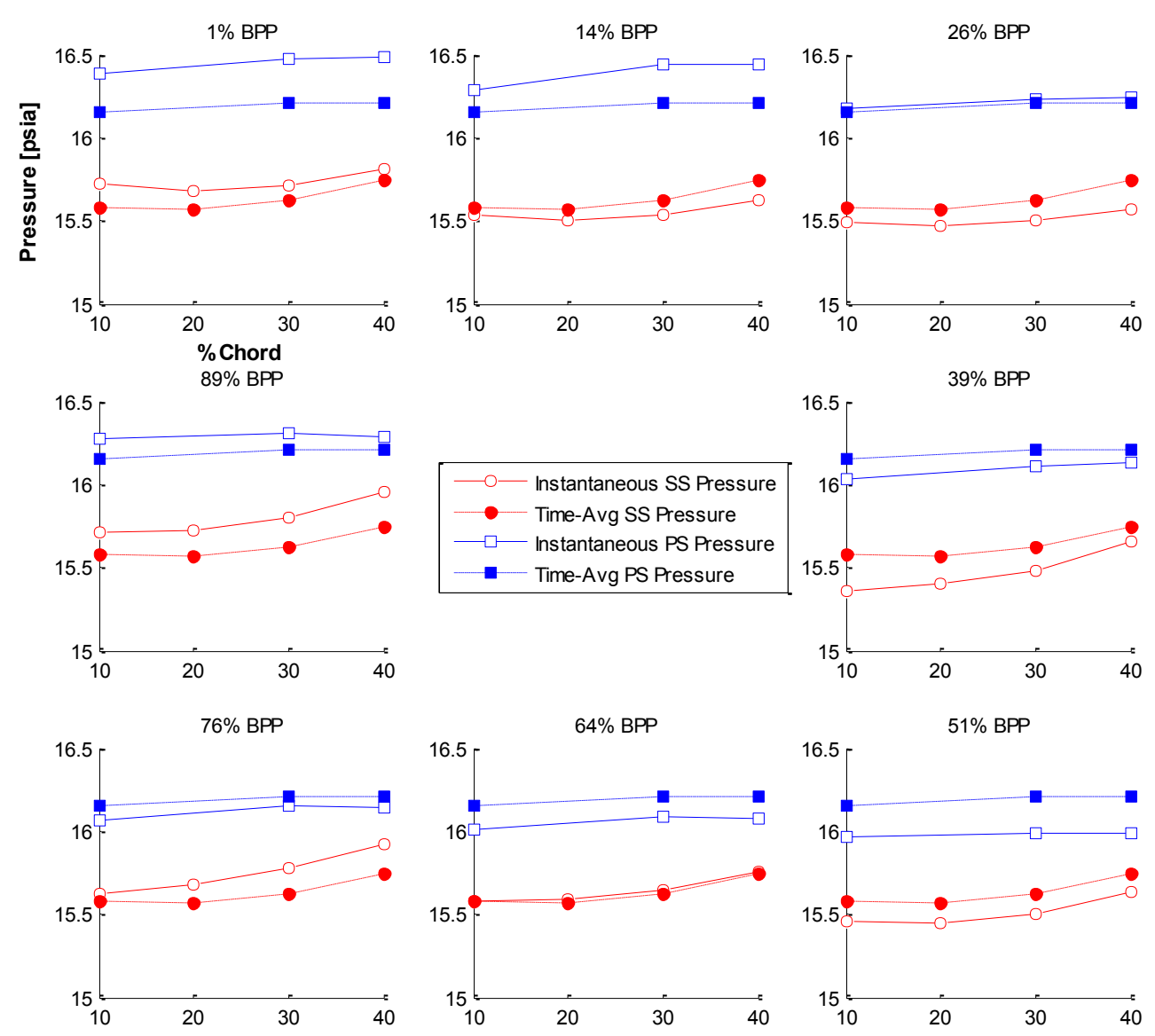

Figure 4.42: Stator Kulite blade loading as a function of rotor position, 50\% Span HL. 

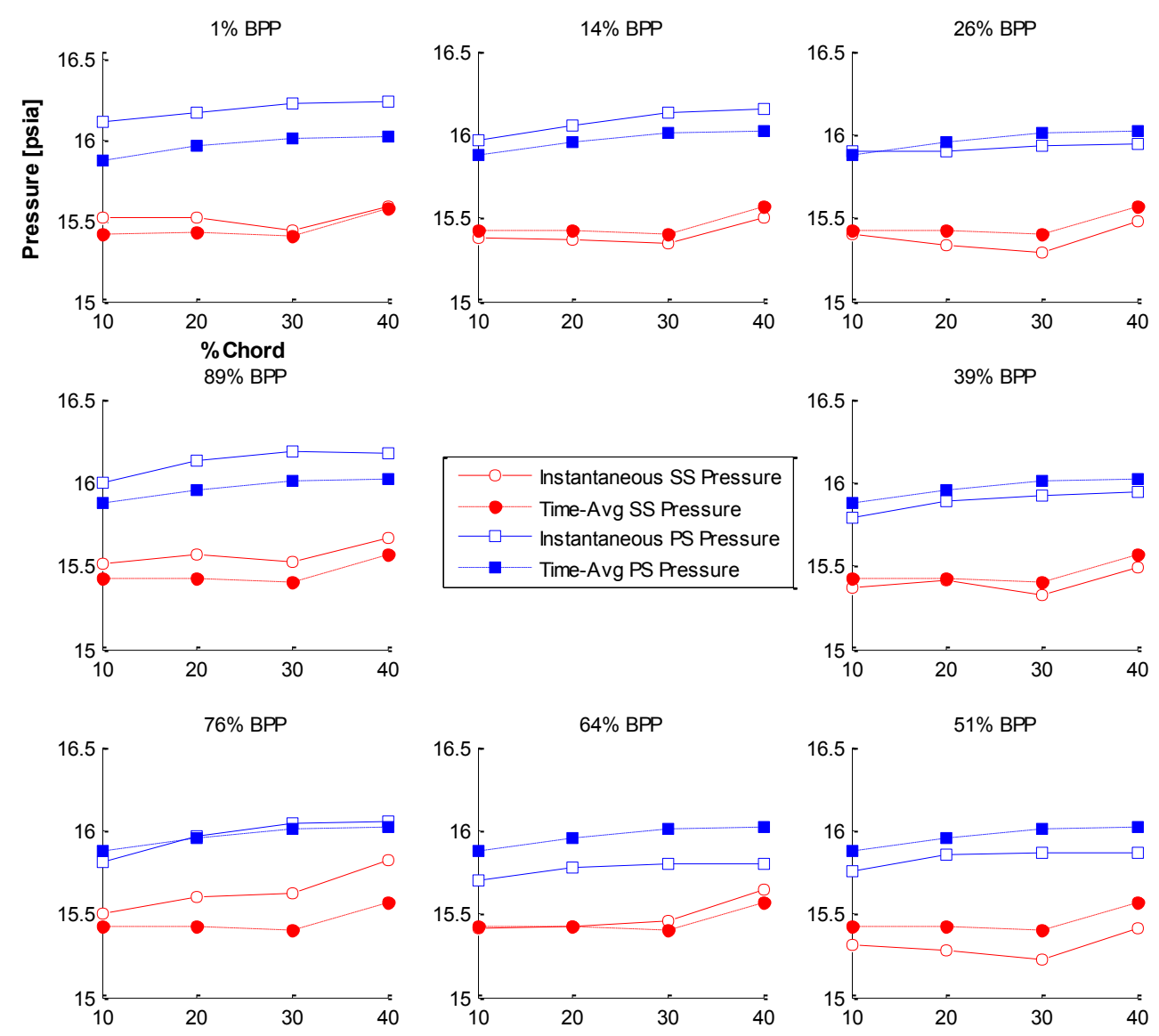

Figure 4.43: Stator Kulite blade loading as a function of rotor position, 80\% Span NL. 

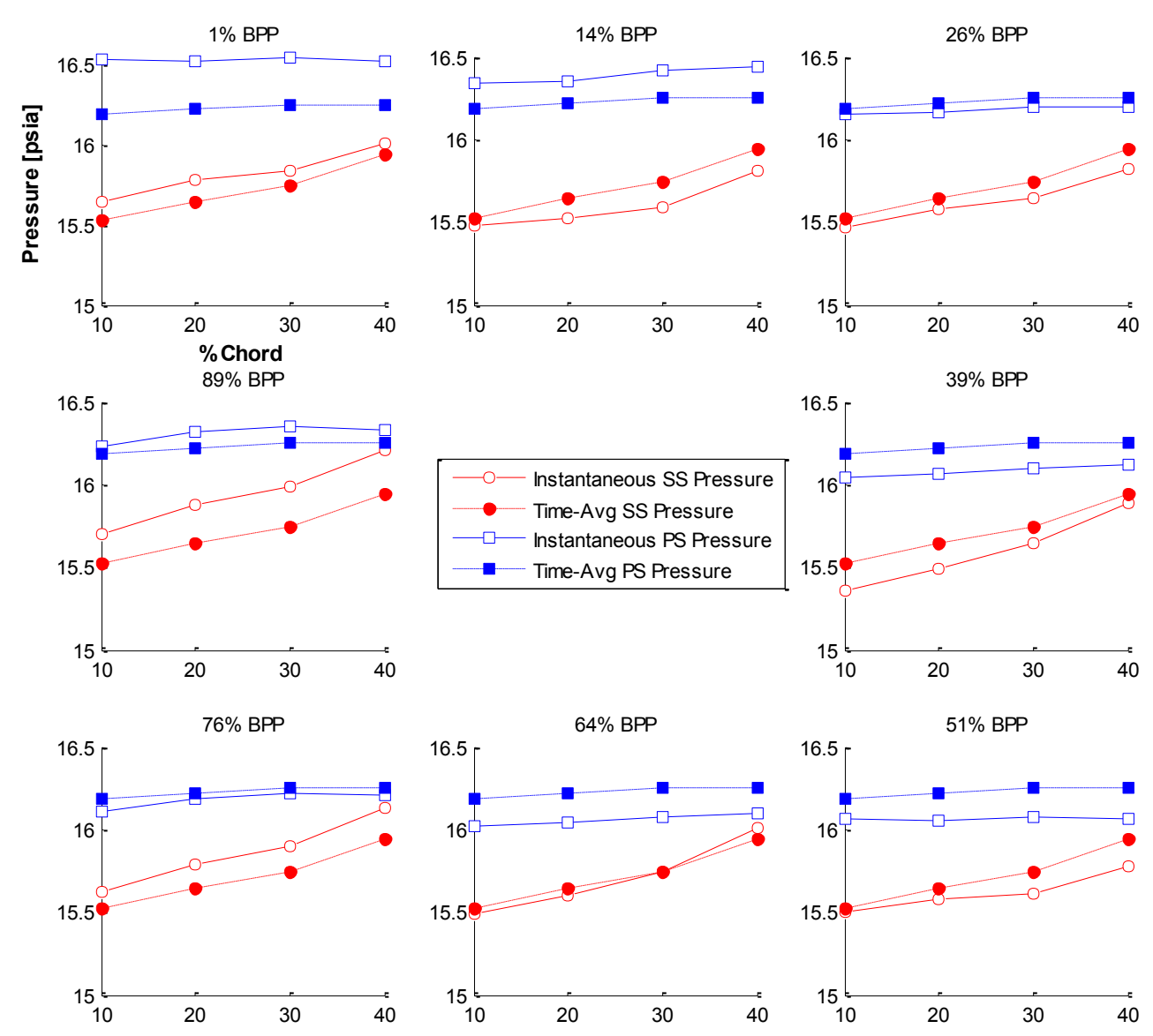

Figure 4.44: Stator Kulite blade loading as a function of rotor position, 80\% Span HL.

During high loading, there seems to be less of a downward slope in pressure on the pressure side sensors, indicating a higher incidence from upstream flow to the stator blade. This is also suggested by the larger pressure difference, especially at $10 \%$ chord, between pressure side and suction side. The pressure side curve seems to flatten out at high loading, whereas the suction side curve becomes steeper as a function of chord. This increase in angle of attack is validated by hotwire data - there is an average increase in $\alpha$ of approximately 8 degrees between NL and HL. There is also a larger increase in rotor wake 
at high loading, visible by the larger deviations from average at both $50 \%$ and $80 \%$ span, although it is more noticeable at higher span.

Figure 4.45 through Figure 4.48 are analogous to the 4 figures shown previously, except they display FT of 600 revolutions of data at each sensor in the stator section. Figure 4.45 shows spectra obtained at NL for pressure side Kulites, and Figure 4.46 shows FTs of the same sensors at HL. Figure 4.47 and Figure 4.48 show spectra of the suction side sensors at the same loadings. There is a "missing" plot in the pressure side figures due to the malfunctioning Kulite at $20 \%$ chord, $50 \%$ span.
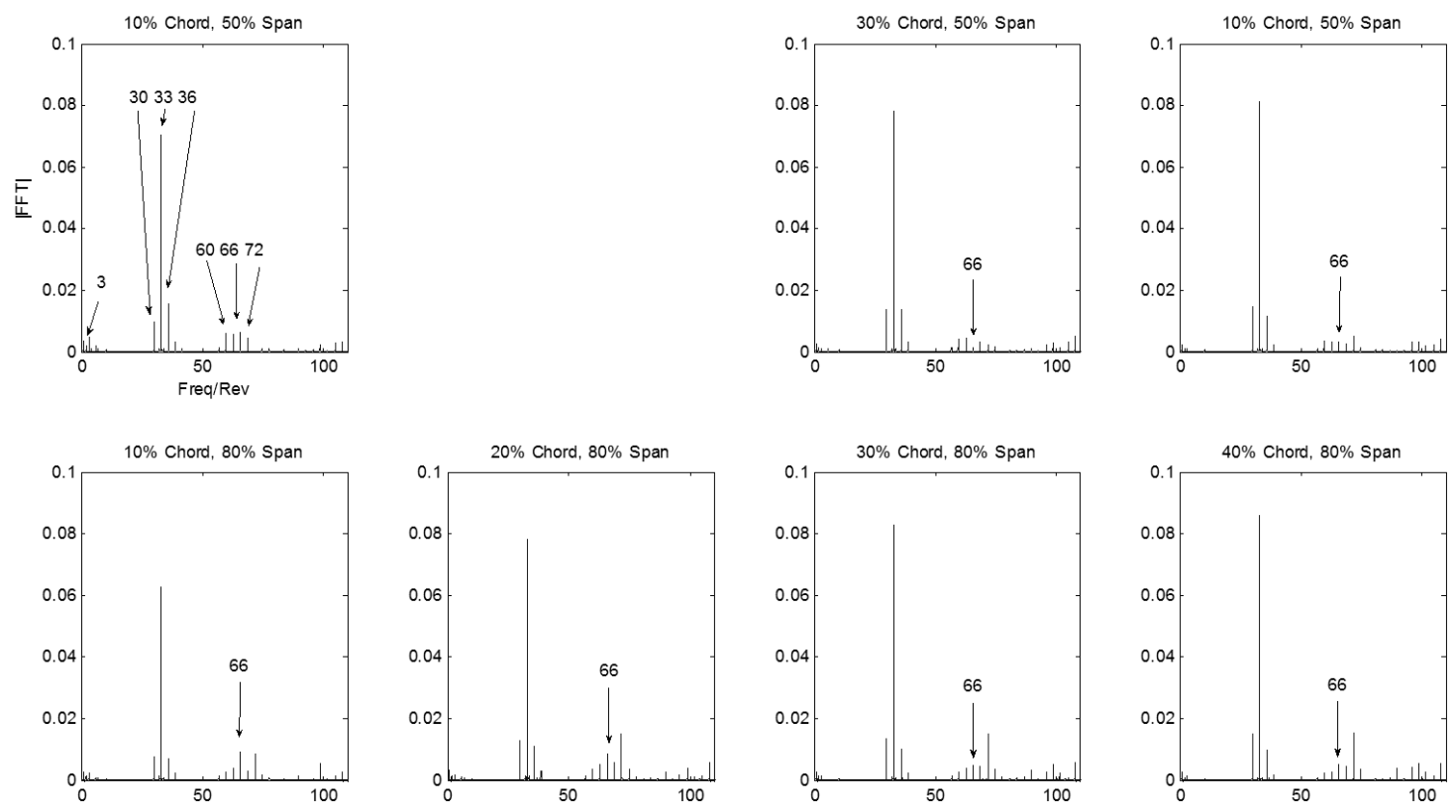

Figure 4.45: FT Spectra of pressure side Stator Kulites, NL 

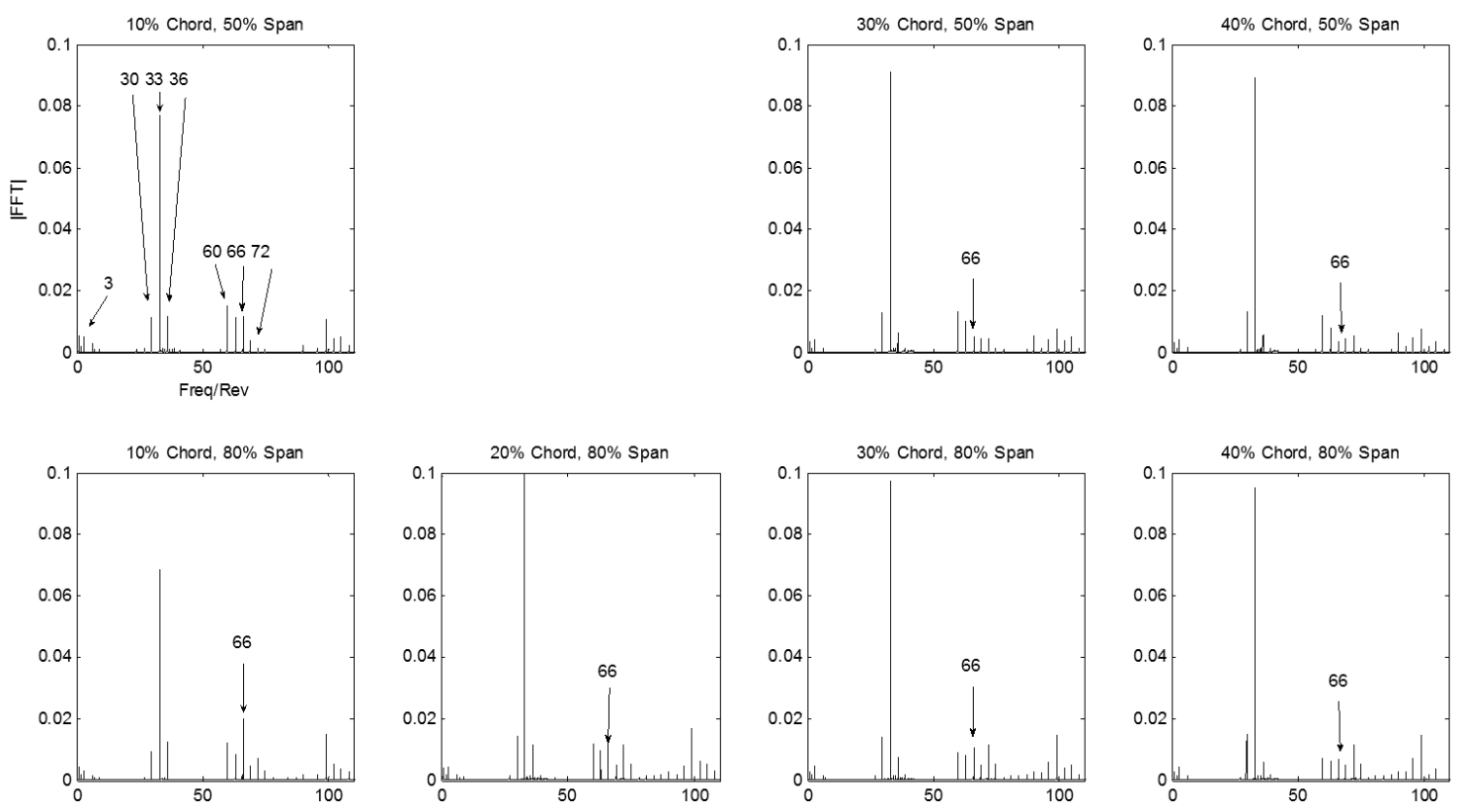

Figure 4.46: FT Spectra of pressure side Stator Kulites, HL
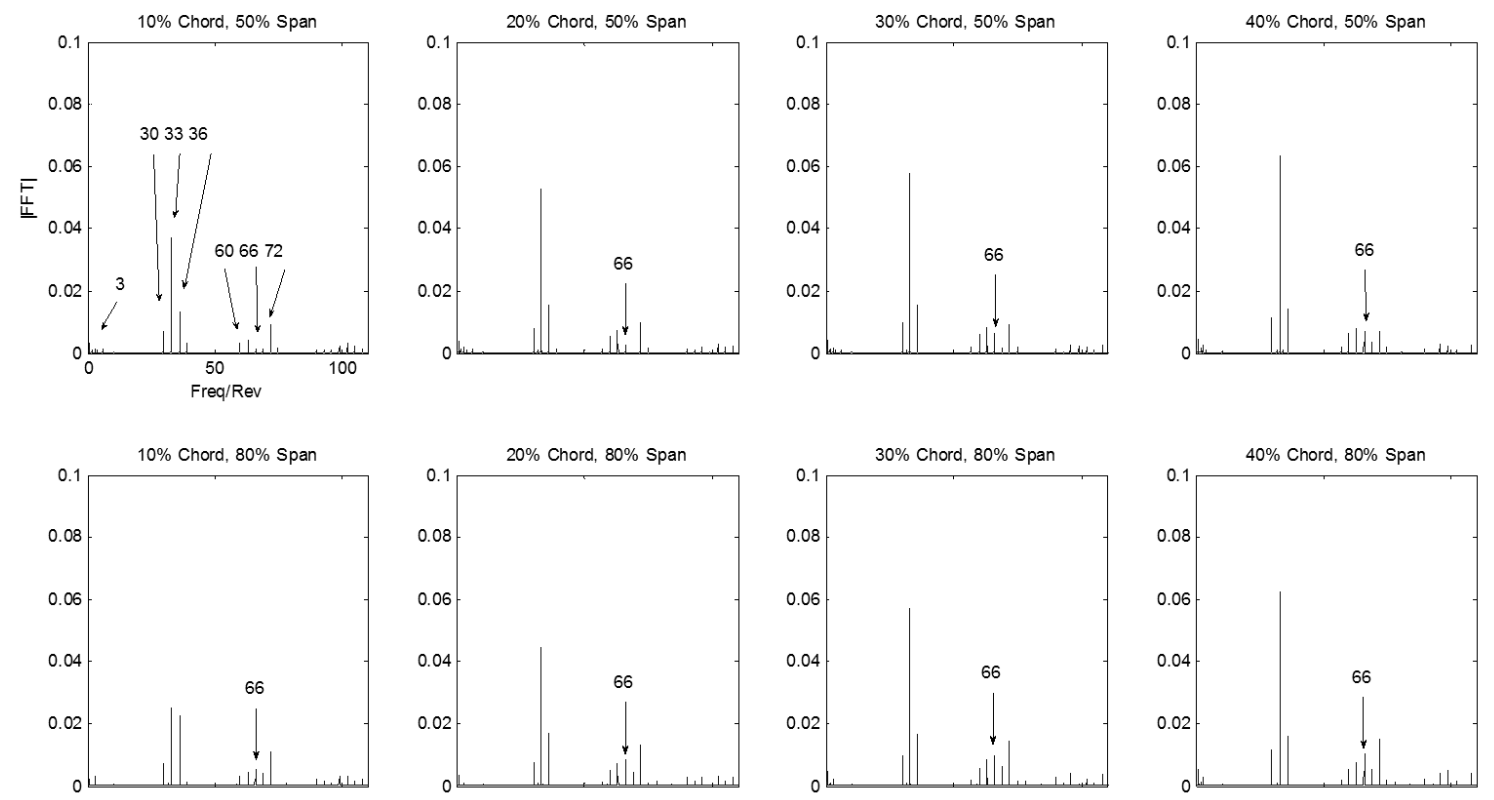

Figure 4.47: FT Spectra of suction side Stator Kulites, NL 

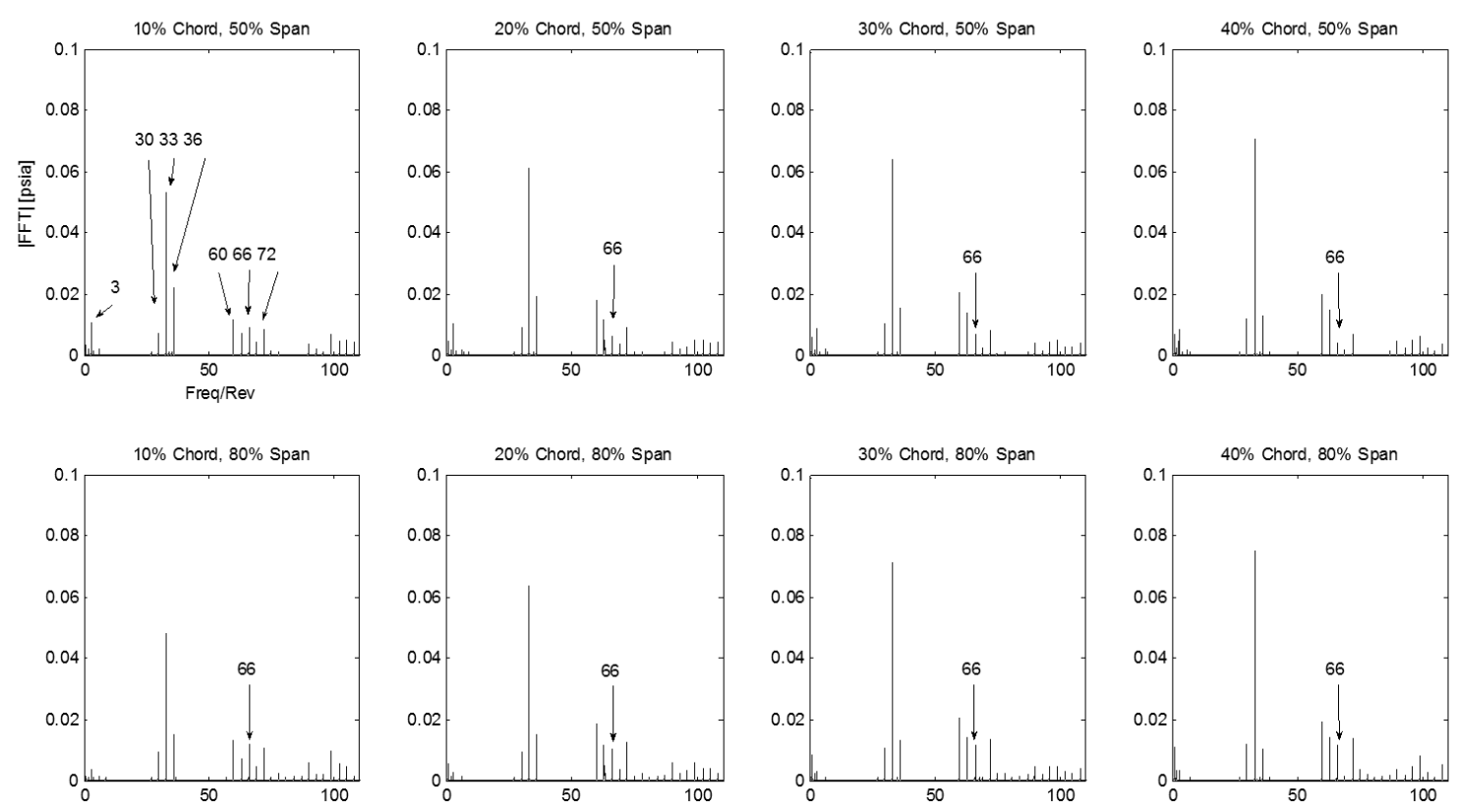

Figure 4.48: FT Spectra of suction side Stator Kulites, HL

There is a very visible correlation between the $1^{\text {st }}$ harmonic of R2 BPF (33/rev) and loading - the strength of this spectral component increases at HL over $100 \%$ at some sensor locations compared to NL. However, the $2^{\text {nd }}$ harmonic of R2 BPF is often dwarfed by R1 or R3 (72 and 60, respectively). It also appears that the $1^{\text {st }}$ harmonic of R2 BPF is lower at $10 \%$ chord than at $20 \%$ chord. It appears to grow in the downstream axial direction, picking up amplitude, especially in suction side Kulites. This could be due to frequency attenuation throughout the stator passage. This will be important when analyzing the stator section Kulites for traces of $\mathrm{R} 2$ vibration. It is also much stronger on pressure side Kulites rather than suction side Kulites. The characteristic 3-beat spectra seems to have strength only at HL for the suction side $50 \%$ Kulites, but is not very evident otherwise. Additionally, the $3^{\text {rd }}$ harmonic of BPF is stronger at PS $80 \%$ span sensors than all other positions. 


\section{CHAPTER 5. EVIDENCE OF R2 VIBRATION IN UNSTEADY FLOW FIELD MEASUREMENTS}

\subsection{Motivation}

Currently, to detect vibration during an EO forced response condition, it is necessary to instrument a stage or a compressor with costly instrumentation such as NSMS laser probes or blade-mounted strain gauges or pressure transducers. It would be helpful to develop techniques that would give cheaper case-mounted pressure transducers the ability to detect EO forced response during compressor operation. It would be helpful as a realtime diagnostic tool or simply as a backup to more complex vibration detection instrumentation, as it would be much cheaper to instrument a compressor with case mounted pressure sensors than instrumentation on rotating machinery. It is difficult because the pressure fluctuations due to EO forced response are much smaller in comparison to easily detected pressure fluctuations due to non-EO flutter modes, for example. Additionally, due to the fact that it is an EO forced response, pressure fluctuations are Doppler shifted to frequencies already heavily influenced by blade pass frequency (BPF) spectra, making the distinction between BPF and forced response pressure components. 


\section{2 $\underline{\text { LINSUB Calculations }}$}

LINSUB is a linear 2D flat blade cascade aeromechanics solver created by Whitehead (1987) that can be used to predict the unsteady components of the flow field within rotating turbomachinery given several inputs and will be used to predict the magnitude of the unsteady pressure component from Rotor 2 vibration. It outputs the absolute and fluctuating components of the flow field, such as lift, pressure, moments, etc. related to bending, torsion, chord wise bending, and wakes/gusts. In this case, it is of interest to calculate the unsteady pressure waves traveling upstream and downstream from a vibrating blade in torsion. There are several calculated inputs to the code, and they are summarized in Table 5-2. LINSUB was compiled in FORTRAN 44 and run based on these inputs.

Table 5-1: List of LINSUB Inputs

\begin{tabular}{|l|l|}
\hline Input Variable & Value \\
\hline Number of Control Points & 20 \\
\hline Spacing to Chord Ratio & 0.767 \\
\hline Stagger Angle in Degrees & $49.6^{\circ}$ \\
\hline Mach Number & 0.341 \\
\hline Reduced Frequency Based on Chord & 1.74 \\
\hline Elastic Axis Position & 0.37 \\
\hline Interblade Phase Angle in Degrees & $-120^{\circ}$ \\
\hline Number of Airfoils & 33 \\
\hline
\end{tabular}

The number of control points was chosen as 20 was based on previous examples of LINSUB runs. All of the variables input to the code were taken from conditions calculated at the tip of rotor 2 . The spacing to chord ratio was calculated from a chord of $2.977 \mathrm{in}$. for $\mathrm{R} 2$ at the tip. Stagger angle was taken to be $49.6^{\circ}$ from BD76 at a radius of $11.935 \mathrm{in}$.

$$
\text { Spacing }=S=\frac{P}{33}=\frac{2 \pi r}{33}
$$




$$
\frac{\text { Spacing }}{\text { Chord }}=\frac{\frac{2 \pi 12}{33} \text { in }}{2.977 \text { in }}=0.767
$$

Relative Mach number was taken from a run of BD76 throughflow analysis code at 3712 RPM, which was the average rotational speed at which the blades respond in $1 \mathrm{~T}$ at High Loading. This speed was chosen roughly to split the resonance band and give a good approximation of flow conditions. The relative Mach number at $99.8 \%$ span was 0.341 .

To calculate reduced frequency based on chord, Eqn. 5-3, $\omega$ was assumed from an average frequency of the blade response at $\mathrm{HL}$ to be $2727 \mathrm{~Hz}$, and flow velocity, $v$, was calculated from Eqn. 5-4.

$$
k=\frac{\omega c}{v}
$$

Static temperature was approximated from speedline data taken at $68 \% \mathrm{~N}_{\mathrm{c}}$ near resonance to be $541.9^{\circ} \mathrm{R}$.

$$
v=M \sqrt{\gamma R T}
$$

The elastic axis position is 0.37 based on the response shape of R2 1T. Interblade phase angle was calculated using Eqn. 5-5, where $\mathrm{j}$ is chosen such that $|\sigma|$ is minimized, and the calculation is shown in Eqn. 5-6.

$$
\begin{array}{cc}
\sigma_{\text {rotor }}=-2 \pi \frac{m}{N B}+2 \pi j \quad \text { where } \quad j \in(\ldots,-2,-1,0,1,2, \ldots) & \text { Eqn. 5-5 } \\
\sigma_{\text {rotor }(\text { deg })}=-360^{\circ}\left(\frac{44}{33}\right)+360^{\circ}(1)=-\mathbf{1 2 0}^{\circ} & \text { Eqn. 5-6 }
\end{array}
$$


The upstream pressure coefficient due to torsion is $1.6488-0.18 \mathrm{i}$, and the downstream pressure coefficient due to torsion is $-3.2502+0.36 \mathrm{i}$. The imaginary part of the vector is used to calculate the unsteadiness of the pressure wave, as shown in Eqn. 5-7:

$$
P_{c}=\operatorname{Re}\left(\vec{v} e^{i \omega t}\right)
$$

The maximum pressure component occurs at $t=0$, and thus, only the real part of the output is necessary to get an approximation of the largest expected pressure wave resonating from the blade during torsion. The coefficients need to be multiplied by the flow density and the square of the relative velocity component, or $\rho_{o} W_{o}^{2}$, which is the dimensionalizing factor for pressure. Then, they will need to be multiplied by the torsion angle, $\phi$, which was taken to be 0.0118 radians, an averaged value for all blades during $\mathrm{HL}$ from NSMS data assuming an elastic axis position of 0.37 . This coefficient was calculated to be 0.00689 , and $\rho_{o} W_{o}^{2}$ was $2.567 \mathrm{lbf} / \mathrm{in}^{2}$. By solving Eqn. $5-8$, it was calculated that the maximum upstream pressure component would be 0.0424 psi and the maximum downstream pressure component would be 0.0848 psi.

$$
P=P_{c}\left(\rho_{o} W_{o}^{2}\right) \phi
$$

By taking into account the stated manufacturer's uncertainty in the XTL-140 Kulite pressure transducer alone, it could be argued that the pressure response from the torsion mode is so small that it could not be detected by the pressure transducer on the casing. The XTL-140 and LQ-062 specifications from Kulite state that the uncertainty can be from $0.1 \%$ to $0.5 \%$ of the full range of the transducer, which corresponds to $0.005-0.025 \mathrm{psi}$. This 
uncertainty does not include any uncertainty or noise introduced by connectors, cables, Precision Filter amplifier equipment, or the PXI analog to digital conversion that would make it even harder to detect the pressure waves. Despite this, because of the fact that the analysis is inherently $1 \mathrm{D}$, there may be important unmodeled 3D effects that could dampen or amplify the pressure components that would allow them to be captured by a pressure transducer. Also, this method only calculates the upstream and downstream traveling pressure components, not the radial pressure, which the casing mounted Kulites were designed to detect. Also, regardless of the actual magnitudes of the calculated pressure waves, the analysis indicates that the pressure waves traveling downstream should be about twice the amplitude of the upstream traveling waves, indicating that the Kulites mounted in Stator 2 would have a better chance at measuring the pressure waves than casing Kulites mounted upstream of R2.

\subsection{Data Processing Strategy to Detect Blade Vibration}

To detect the very small pressure generated by R2 1T, several analytical data processing methods had to be developed. The most difficult issue was the removal of background noise/pressure information that was not pertinent to forced response. Additionally, complex data handling algorithms needed to be developed to handle the large volumes of unsteady binary data and divide it into useful portions that could be used for vibratory analysis. 


\subsubsection{Reconciling Revolutions of Transient Data}

Each set of unsteady pressure and hotwire data was acquired for a certain number of seconds, a variable that could be selected in LabView before the start of each data acquisition period. For example, with the casing Kulites, there were a total of 26 channels of data recorded -25 channels of Kulite pressure data, and 1 channel that would record the 1/rev output from the encoder mounted on the output shaft of the 3 -stage gearbox. For every revolution of the compressor, the $1 /$ rev channel would emit a short $0-5 \mathrm{~V}$ square wave that would signify the compressor had rotated 360 degrees. Depending on the type of run, the data were acquired either at a steady RPM or in an accelerating or decelerating ramp through resonance. Usually, when data was acquired at a steady compressor RPM, the data channels were recorded from 8-12 seconds, resulting in over 500 revolutions of data. For the sweeps through resonance, 1200-1800 revolutions of data were acquired. For example, if there were 1,800 revolutions of data for 25 channels, there would be a total of 45,000 unique variables created and saved as a MATLAB readable file. Each one of the variables would be a unique length, depending on sample rate. Because the variable frequency drive that controls the motor speed can hold speed to a tolerance of approximately $+/-0.1 \%$, the length of each variable, even on a steady run, would vary by $+/-2$ sample points at a sampling rate of $300 \mathrm{kHz}$. During a sweep, when compressor speed would change, each revolution of data would vary in length up to 50 sample points (usually the length of one blade pass period) by the end of the data acquisition. Thus, each of the 45,000 unique revolutions are phase shifted so that the first visible blade pass pressure signature corresponded to blade number 1 , the same blade that NSMS calls blade number 1 . The 1/rev signal normally occurs roughly at the position of blade 12 , so this time shift was 
necessary to facilitate data processing. Each of these 45,000 revolutions of data was then divided again by 33 - the number of blades in Rotor 2 - resulting in approximately 1.48 million vectors. At this point, the data from a given run could be analyzed on a revolutionby-revolution basis, or also a blade-by-blade basis.

For acquisition at steady speed, it was easy to set up a code that would read in the $1 /$ rev channel, and based on where the square wave would occur, set a cutoff voltage for which each of the revolutions of data were cut out into separate vectors for each channel. The compressor speed would vary slightly through each run, which, depending on the sampling rate of the data, would result in vectors of slightly varying lengths, different by usually 1-2 samples.

However, for a sweep through resonance, it was particularly challenging to account for revolutions of data that would vary significantly in length between the beginning and end of the run. It was impossible to create ensemble-averages of the data without either stretching or shrinking the waveform, and ordinary matrix operations became difficult. Therefore, all data were split into MATLAB-based "structures" labeled according to channel and revolution number, starting at 0 for channel number and 1 for revolution number. Revolution 1 was usually of truncated length because of the fact that the data acquired did not start exactly with the $1 /$ rev square signal. Figure 5.1 shows the calculated time between $1 /$ rev pulses for a run through approximately 1400 revolutions of data accelerating through resonance. The stepwise nature of the plot is due to the fact that the change in RPM of the compressor was slow enough that the time between $1 /$ rev pulses from one revolution to the next was only a fraction of the sampling rate, which happened to be $225 \mathrm{kHz}$. This made it difficult to calculate RPM, which was an important parameter 
that would be used to analyze the unsteady data. Therefore, a Least Squares Fit (LSF) line was calculated based on the $\Delta t$ from the time between pulses, and the equation for this line was used to calculate the speed of the compressor at every sample point using Eqn. 5-9.

$$
\Omega=\left(\frac{1}{\Delta t}\right) 60
$$

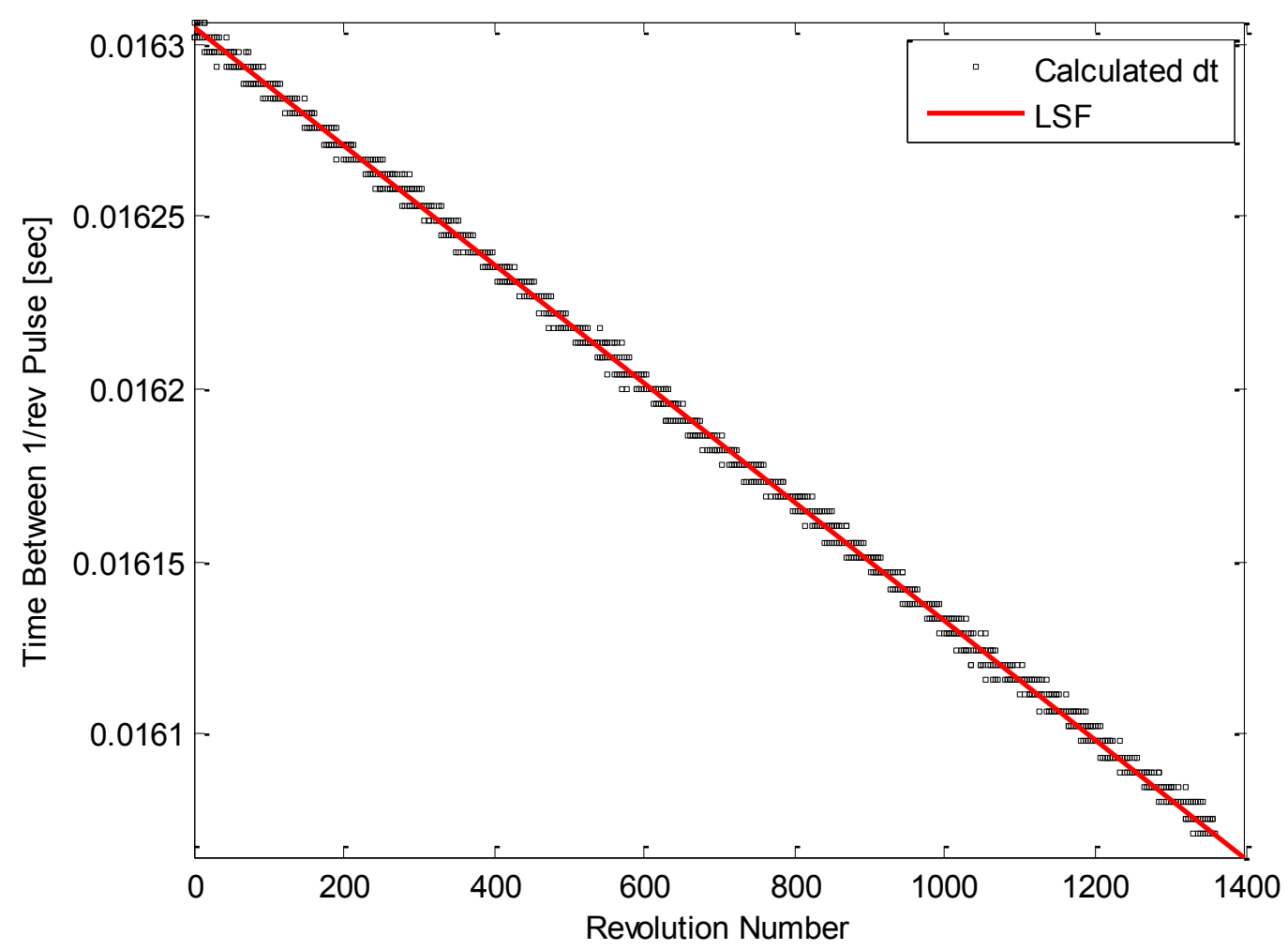

Figure 5.1: Calculated time between square wave pulses from the $1 / \mathrm{rev}$ channel as a function of revolution number for a data run accelerating through R2 1T.

Many different methods of looking at the rotor blade pass pressure signature shape and size were utilized. Initially, just a qualitative approach of looking at the blade pass shape was used to look for trends in the data and perhaps be able to gauge a difference in overall amplitude or shape when the rotor was vibrating. Figure 5.2 is a visual representation of what the characteristic blade pass signatures look like, divided into 33 
equal length vectors representing each $\mathrm{R} 2$ blade. This is also useful to illustrate how the blade pass signatures were parsed into vectors in MATLAB.

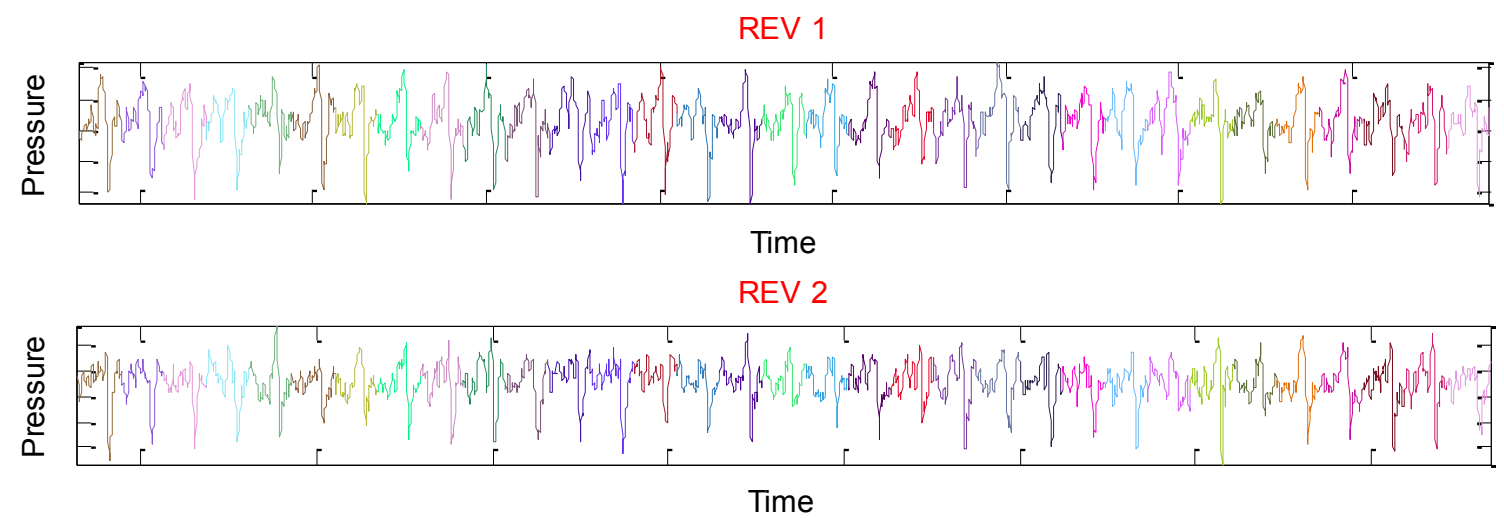

Figure 5.2 Sample blade pass signatures from casing Kulites for each revolution.

Taking an individual blade, measured by a single pressure transducer, and averaging all of the revolutions of data together for that blade, an "ensemble average" pressure trace can be calculated, shown by the red pressure trace in Figure 5.3. However, since the compressor is accelerating or decelerating, the shape of the blade pass signature will change by shrinking or expanding in time in proportion to the change in compressor speed, and thus the amount of time the blade is in front of the transducer. Therefore, this method of characterizing the shape of the blade pass is difficult unless the amplitudes are the only characteristic under consideration. 


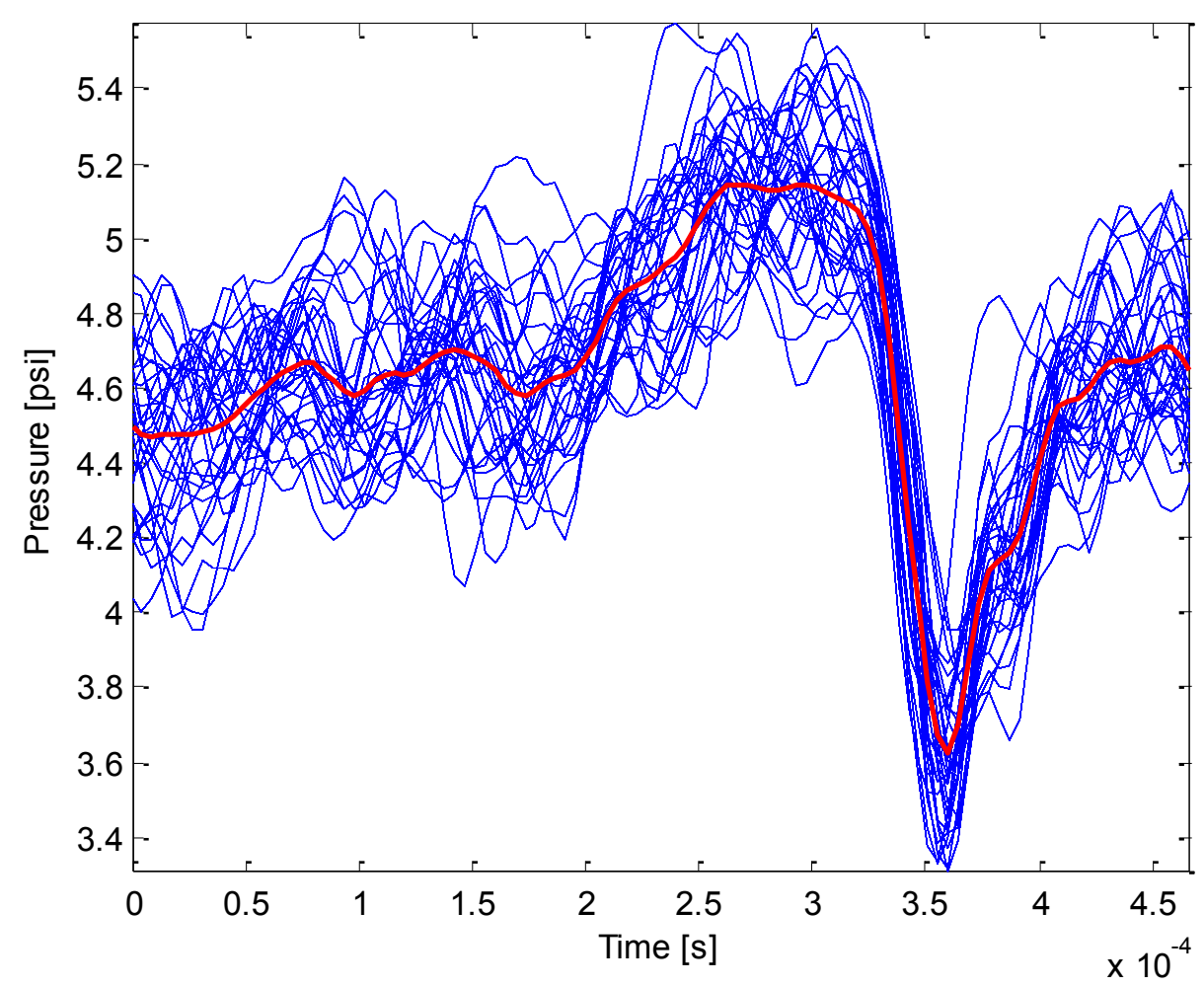

Figure 5.3: Example ensemble average of one blade pass from a revolution of Kulite data, HL, 3680 RPM, casing Kulite.

Because the rotor blade count for each rotor differs by three blades, (the counts for R1-R2-R3 are 36-33-30, respectively), there is a characteristic "3-beat" modulation to the pressure signal of each revolution that is evident in the signal. Because this modulation changes the overall amplitude of each blade pass by a large amount, it is difficult to use an amplitude-based comparison method when analyzing the blade pass signatures. Figure 5.4 shows two graphs. The one on the left shows blade number versus compressor speed. As each revolution is recorded, the blade with the largest pressure amplitude difference between the minimum pressure and maximum pressure is noted and recorded. As the sweep progresses, it becomes obvious that the plot tends to show a characteristic 3-beat pattern, 
where the maximum pressure signals tend to be driven more by the interference or reinforcement from rotor-rotor interactions rather than any torsion vibrational response. This analysis was performed for all 25 pressure sensors in the casing, and they all tend to follow the same pattern. A graph of maximum blade displacement amplitude as measured by the NSMS system, is shown on the right for comparison of which blades are the highest vibrational responders.
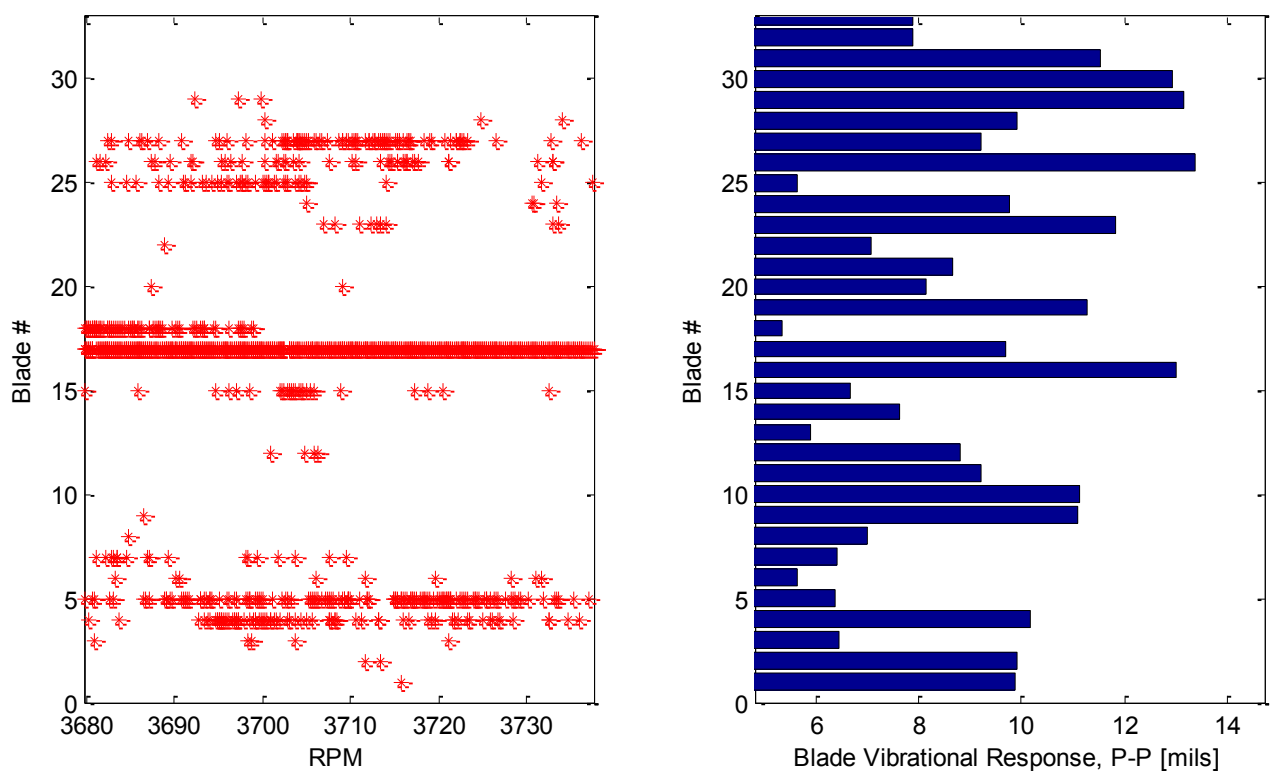

Figure 5.4: Comparison of blade-to-blade method of maximum blade pass pressure amplitude, NL decel Casing Kulites.

This same method was also carried out with simply finding the blade pass with the largest standard deviation over an ensemble average, with similar results that were inconclusive. Therefore, another method for evaluating the pressure signal in a time domain was necessary. By using a method that would weight the difference between the waveform when it is out of resonance with the waveform during resonance, one might be able to spot a noticeable, repeatable change in the pressure wave when the rotor is vibrating. 
By analyzing the entire pressure revolution waveform as a whole, one might be able to detect changes in the time of arrival of the pressure waves emanating from the tip of Rotor 2, similar to how NSMS is able to detect vibration using optical methods. By taking the waveform of a revolution of data from one sensor, while the blade is not undergoing torsion, and comparing this waveform to every subsequent waveform, a cross correlation, cross covariance, or standard deviation could indicate the level of similarity of the waveforms in and out of resonance. Because the length of the revolution vector is continuously changing, it is necessary to do some basic signal manipulation for this analysis.

\subsubsection{PXI Lag Offset}

During normal unsteady data acquisition at constant compressor speed, there seems to be a slight lag in data acquisition when the PXI box changes from taking data on one card or the other. This was discovered when processing unsteady data for stator Kulites. To individually take out each blade pass signature and overlay them on top of each other for comparison, one revolution of data was simply divided into 33 equal-length vectors, each one corresponding to one blade. This brings to light a shift in the data, such that the blade passes were not lining up with each other on each successive revolution. The lag grows with duration of acquisition. The lag was very small — fractions of one data sample at $300 \mathrm{kHz}$ sample frequency. 

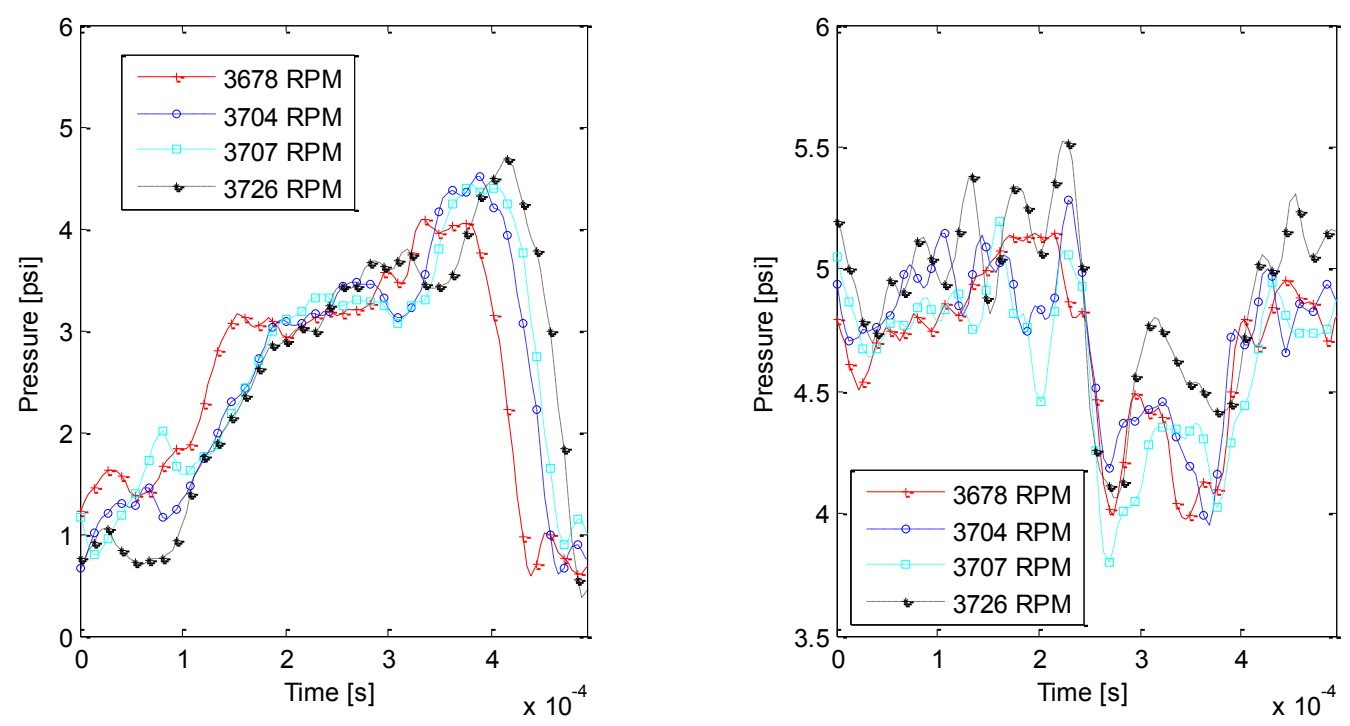

Figure 5.5: Blade 13 passage seen by casing Kulites acquired on different PXIE cards.

It is worth noting how the issue was narrowed down to the switch between the two PXI-E cards in the PXI-1073 box. At first, this issue was discovered by simply plotting the very first 100 point of each revolution, as the data was cut by the $1 /$ rev signal. By making a plot of the first portion of each successive revolution, one would be able to determine whether or not the data was being shifted at all. A MATLAB script titled "PXI_Lag_Test.m" was written to do this. It would also take each successive group of 100 revolutions of data and change the color of the vector, so that it was easy to discern movement/lag/shifting of the data. Figure 5.6 shows 400 successive revolutions shown of a blade passage trace from a casing Kulite. The first 100 revolutions are plotted in red, 101-200 in cyan, 201-300 in black, and 301-400 in blue to show the very gradual offset as a function of blisk revolution number. 


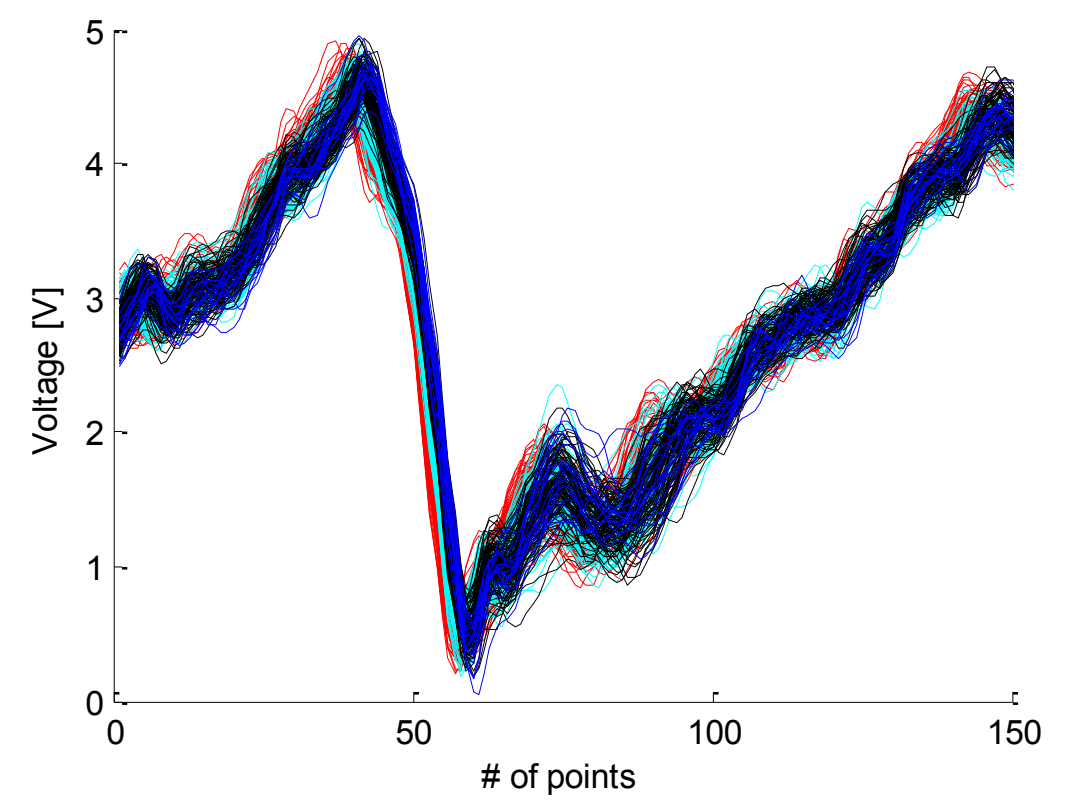

Figure 5.6: 400 successive revolutions shown of a blade pass, illustrating lag offset

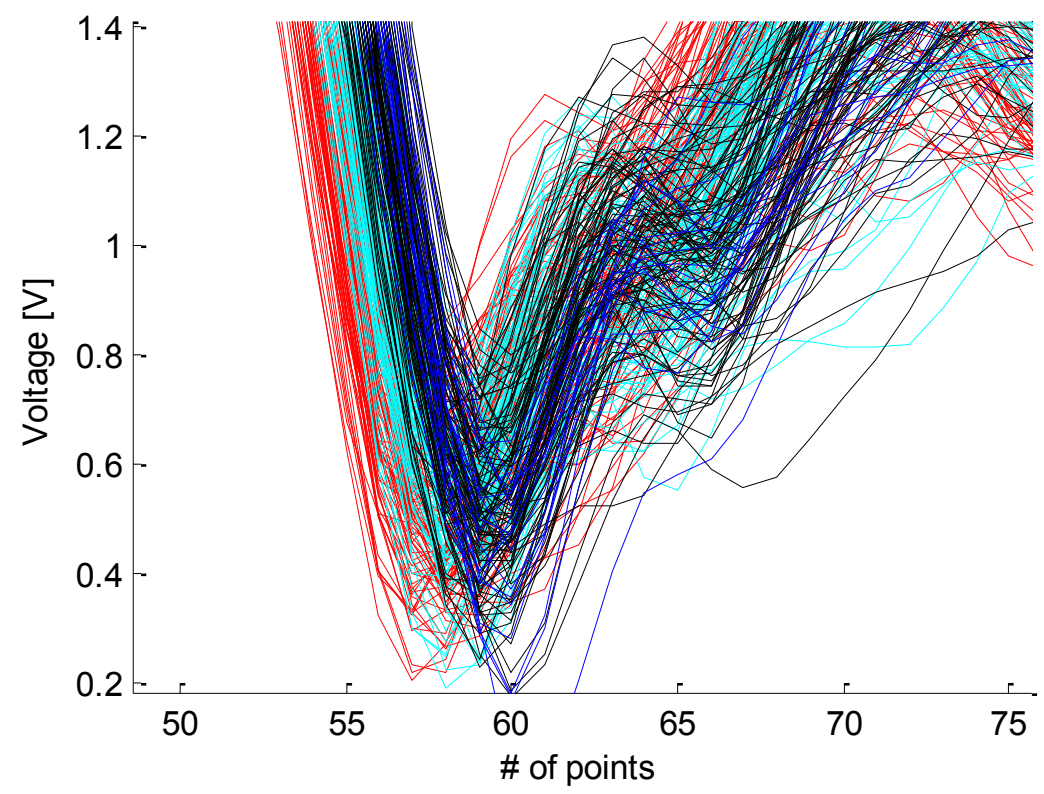

Figure 5.7: Zoomed in image of Figure 5.6. Lag offset per revolution is only a fraction of a sample period. 
From this analysis method, every channel that the PXI box was used to acquire was plotted, to see if there was any difference in the lag from channel to channel. Since this lag occurred at steady compressor speeds, it was not associated with shaft torsion. When all of the channels that were on the same card as the $1 /$ rev there was no offset. Only the channels on the card opposite the 1/rev signal had noticeable lag.

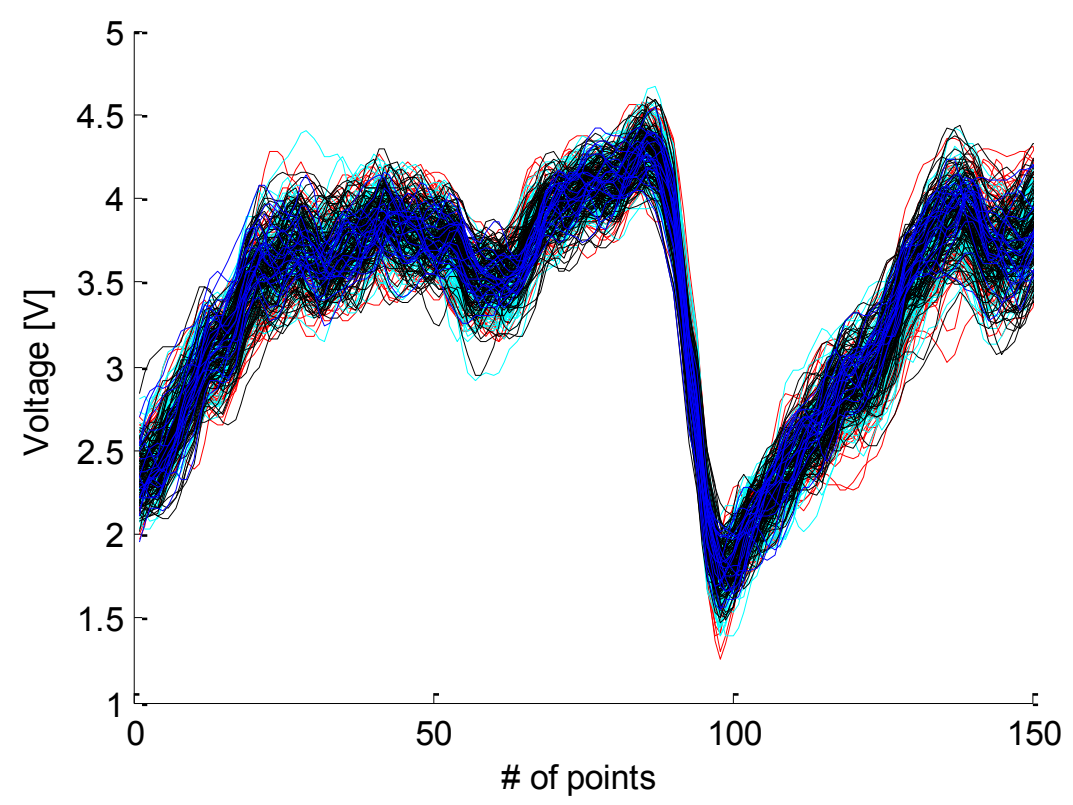

Figure 5.8: Kulite blade passage shape showing multiple revolutions for sensor on same card as $1 /$ rev trigger.

To characterize the offset so that all previously acquired data on the other card could be appropriately time shifted, a sine wave was sent to all 32 channels by using the front-mounted Test Bus BNC input on the PF equipment. Therefore, all channels should be sampling the exact same signal, and the lag between both of the cards should become apparent.

A $42 \mathrm{~Hz}$ sine wave was generated, with an amplified amplitude of approximately $14 \mathrm{~V}$, going from $+7 \mathrm{~V}$ to $-7 \mathrm{~V}$. The frequency of this wave is similar revolution frequency 
of the three stage when it is running at around 50\% speed. By making the amplitude of the sine wave high enough, and the frequency around $42 \mathrm{~Hz}$, the same codes used for Kulite data processing could be used where the sine wave replaced the $1 /$ rev signal.

The tests were designed to determine whether or not the lag was a function of channel number, acquisition time, or sample frequency. So 4 sets of data were taken, using both the PXI box and the Precision Filter amplifier, on all 32 channels simultaneously, using the same Kulite code we have been using for all Kulite data acquisition.

Table 5-2: File name, sample rate, and time of test files used to determine lag offset.

\begin{tabular}{|c|c|c|}
\hline Sample Rate & Acquisition Time & File Name \\
\hline $150 \mathrm{kHz}$ & 8 seconds & $150 \mathrm{kHz} 8 \mathrm{sec} . \mathrm{mat}$ \\
\hline $150 \mathrm{kHz}$ & 16 seconds & $150 \mathrm{kHz} 16 \mathrm{sec} . \mathrm{mat}$ \\
\hline $225 \mathrm{kHz}$ & 8 seconds & $225 \mathrm{kHz} 8 \mathrm{sec} . \mathrm{mat}$ \\
\hline $300 \mathrm{kHz}$ & 8 seconds & $300 \mathrm{kHz} 8 \mathrm{sec} . \mathrm{mat}$ \\
\hline
\end{tabular}

The sine wave data was plotted in the same fashion as Figure 5.6, where 0-400 "revolutions" (in this case, a peak-to-peak of the $42 \mathrm{~Hz}$ sine wave was used as a "revolution") were plotted, and the first few points are shown in order to clearly illustrate the offset. Only 60 samples are shown on the $\mathrm{x}$ axis, and a peak-to-peak sample length at $150 \mathrm{kHz}$ is approximately 3500 samples, and thus, Figure 5.9 is zoomed in on a portion of the sine wave. 


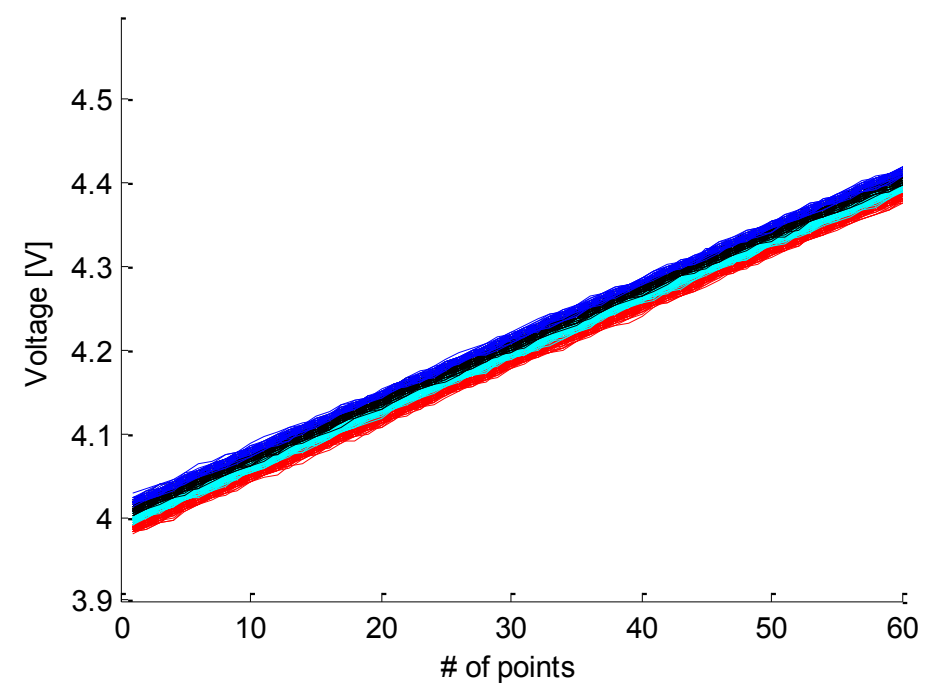

Figure 5.9: Lag offset is also apparent in close up of $42 \mathrm{~Hz}$ sine wave. Channel 17 is plotted, and the " $1 / \mathrm{rev"} \mathrm{sine} \mathrm{wave} \mathrm{used} \mathrm{to} \mathrm{parse} \mathrm{the} \mathrm{signal} \mathrm{was} \mathrm{taken} \mathrm{to} \mathrm{be} \mathrm{Channel} 0$.

The "1/rev" sine wave was taken from channel 0 , which was on the other PXIE6358 card. When plotting a sine wave taken from the same card, there is no lag offset seen, as shown in Figure 5.10.

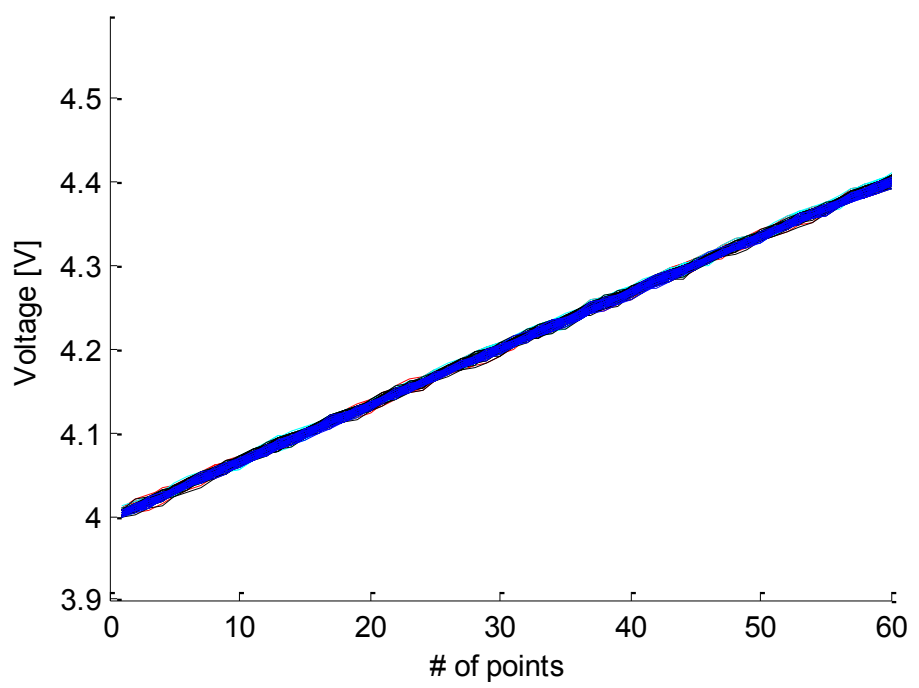

Figure 5.10: Sine wave from channel 0, showing no offset, since it was acquired on the same card as the designated " $1 /$ rev" signal. 
The lag does not depend on sample rate. To determine a value for the lag between each sample, a code was developed named Time_Offset_Calc.m. This code begins with a cutoff input with which it will parse the continuous waveform. It then up samples each "revolution" of data from 10,000 to 100,000 points. This is because the offset is usually a small fraction of a sample, and this is one method to obtain the offset drift. It determines how the "cutoff" index will drift. If the sine wave is moving "backwards", i.e., it is appearing to lag or drift in the negative $\mathrm{x}$ direction, the index at which the 4 volt cutoff will occur will drop. This is not necessarily a constant drift rate because of noise in the generated sine wave. However, this drift as a function of Rev \# is shown in Figure 5.11 with a Least-Squares Fit line applied to the data.

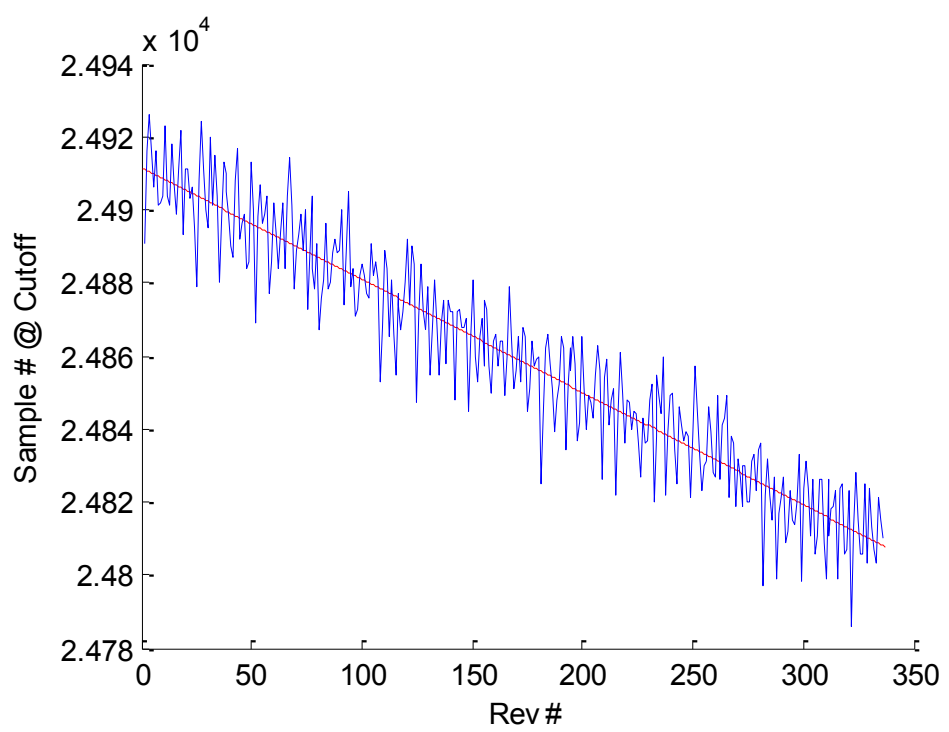

Figure 5.11: Sample number at 6V trigger plotted as a function of Rev \#, with a LSF line applied to the data.

To see the average drift as a function of channel number, Figure 5.12 was generated, and is used to illustrate the drift seen in a few other channels. As can be seen, the LSF lines are very close to being parallel, indicating that the drift is uniform and nearly constant 
across all channels. The time offset was non-dimensionalized by the number of seconds in length of each recorded waveform, and shown in Figure 5.13.

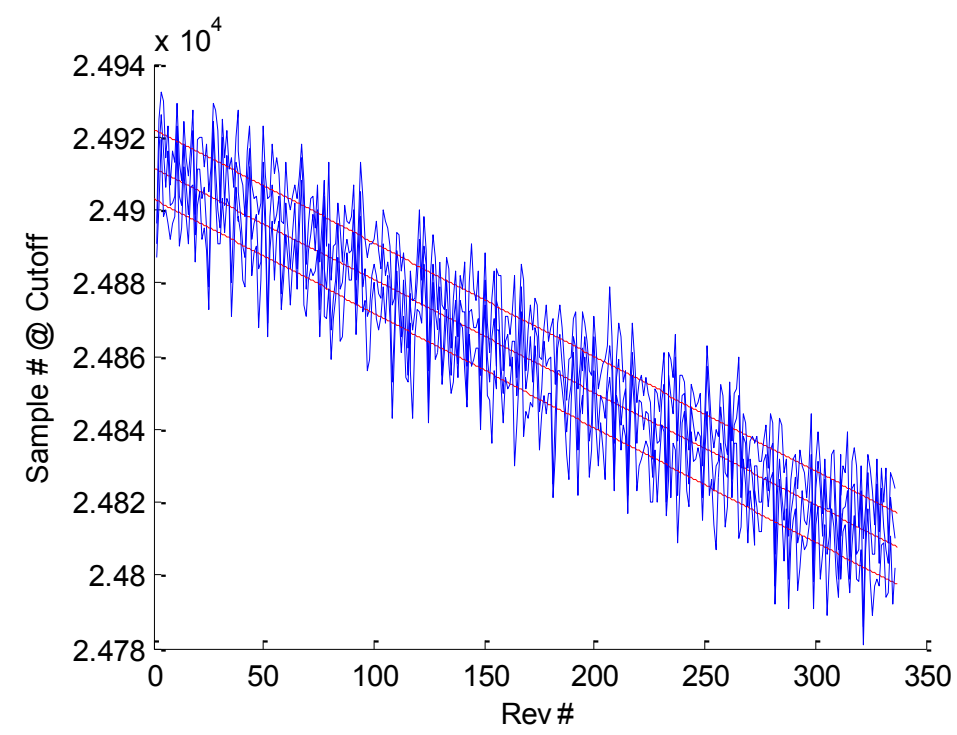

Figure 5.12: Several consecutive channels plotted to show parallel LSF slopes.

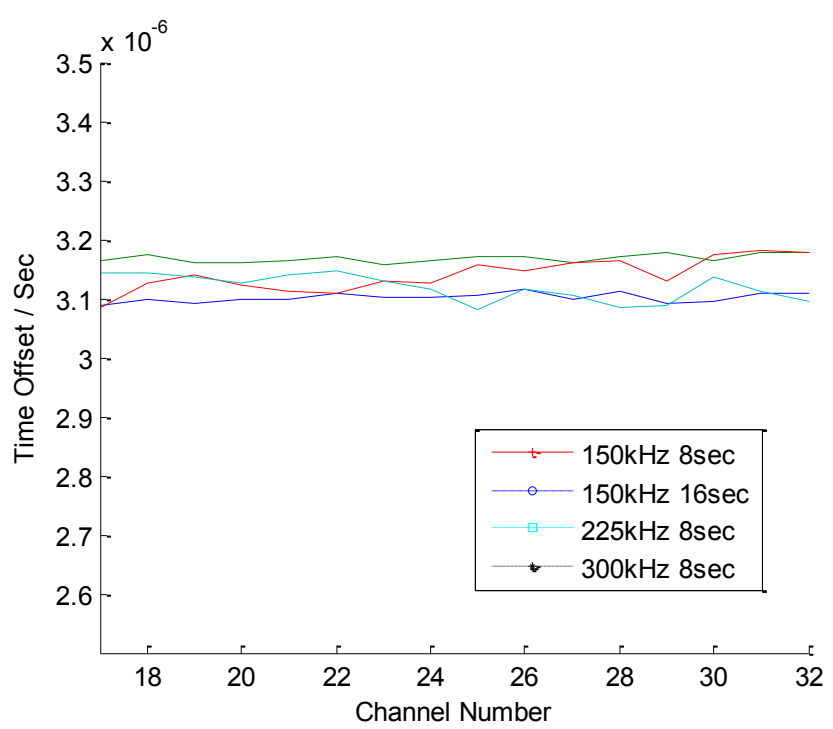

Figure 5.13: Time offset normalized by seconds, plotted as a function of channel number. The drift is nearly constant along all channels and acquisition time. 
The lag offset is a constant value per second no matter what the sample rate is, but that means it has a correspondingly different time offset per sample, as seen in Table 5-3.

Table 5-3: Time offset per sample for different PXIE sample rates.

\begin{tabular}{|c|c|}
\hline Sample Rate (kHz) & Time offset per sample (s) \\
\hline 150 & $2.0671 \mathrm{E}-11$ \\
\hline 225 & $1.3955 \mathrm{E}-11$ \\
\hline 300 & $1.0396 \mathrm{E}-11$ \\
\hline
\end{tabular}

It is possible that the lag issue arises when the PXI box attempts to switch cards, and uses an indicator based on the computer's clock speed to note when the PXI box was done acquiring data on one card and then is ready to acquire data on the other card. This would make it such that the lag changes based on sample rate but is constant over time because it is based on a processor clock timing algorithm.

The best solution to circumvent this issue would be to split the $1 /$ rev so that it is simultaneously recorded by both PXIE cards, and the channels on each card would then be parsed by the respective $1 / \mathrm{rev}$ signal that was recorded at the same time. But in this case, where the data have already been acquired, this is not an acceptable solution. Nor would it be acceptable in the case where the simultaneity between data on channels acquired with different cards is important, i.e. tracking a flow disturbance that moves in the time domain but requires more than 16 channels of data to observe it. 
An algorithm was written to make up for the offset, and relies on a high-definition linear interpolation of a signal in order to work. The lag between the cards would begin to build up every sample, and the lag at each successive sample, based on a constant time delay, would be equal to

$$
t_{\text {new }}=t_{n, \text { sample }}-n * d t
$$

Eqn.

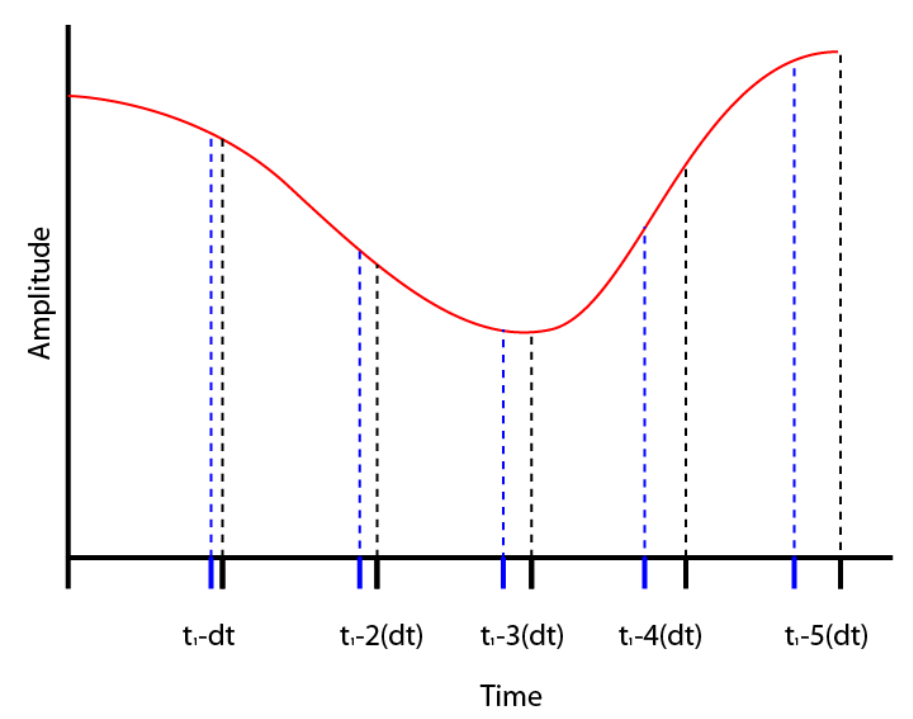

Figure 5.14 Illustration of consecutive addition of time lag offset for each successive sample.

Therefore, a matrix was created at which each sample should have occurred, and linearly interpolate between the original data points to create a new set of data, the offset should be corrected. The code was applied to stator Kulite data and the same $42 \mathrm{~Hz}$ sine wave that was shown earlier, and the results show that the offset works, as long as the correct time lag offset per sample is chosen based on the sampling rate. If other sample 
rates are used for data processing, the lag calculation algorithm must be used on a new set of sine wave data to calculate a new lag offset value. Figure 5.15 shows the same blade passage as seen on the rightmost plot in Figure 5.5, after the PXI lag corrections have been applied, showing no noticeable lag offset.

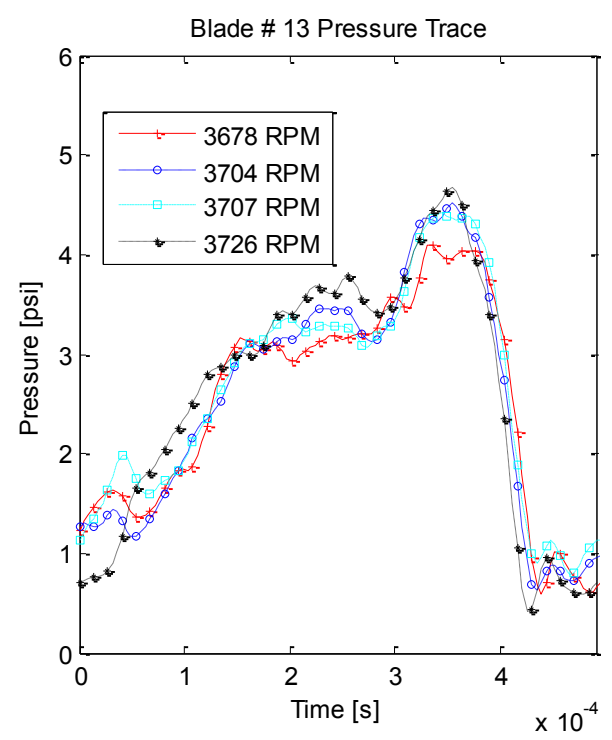

Figure 5.15: Blade 13 passage as seen by casing Kulite, after lag offset corrections.

\subsubsection{Fourier Waterfall Plots}

The practice of recording the vibration of an Engine-Order forced response with Fourier spectra from a stationary pressure transducer is difficult due to the fact that the effect torsion has on the pressure spectra is small in comparison to other spectral components of the signal, such as blade pass frequency and its harmonics. This becomes an even more difficult issue in an embedded stage, due to the frequency spectra from neighboring stages "crowding" the FT. Ideally, the frequency of the torsion vibration would be evident from the Kulite pressure sensors, somewhere buried within frequency components associated with blade pass frequency. 
The Kulite pressure transducers, relative to the rotor, are sitting in a stationary reference frame. Mengle (1990) showed that since the rotor is spinning at a rotation rate, $\Omega$, the vibration frequency of the blades, $\omega$, will be Doppler shifted to a different frequency, $\omega^{\prime}$, based on nodal diameter, $\mathrm{ND}$, and wave number, $\mathrm{m}$.

$$
\omega^{\prime}=\omega+(N D+m B) \Omega
$$

The Nodal Diameter can either be determined from NSMS data or from the difference between stator vanes and rotor blades, since the frequency of vibration is an engine-order (EO) forced response, in this case.

$$
k=N_{\text {Stator Vanes }}-N_{\text {Rotor Blades }}=44-33=11 \quad \text { Eqn. } 5-12
$$

Because of blisk mistuning, the approximate band of blade response varies over a range of $2700-2735 \mathrm{~Hz}$, as measured with NSMS. By choosing $m$ to equal values of $-2,-1$, 0,1 , or 2 , the first few wave modes will be captured. These should be the highest responding modes. When the frequency of interest is an engine-order forced response, the Doppler shift will always shift the forced response to multiples of blade pass frequency. Therefore, the analysis will try and pick out changes in the frequency component of the $33 /$ rev and higher harmonics of the Fourier transform. This may prove difficult because as the compressor speed accelerates, changes in the strength of these components of the signal will also be associated with aerodynamic changes. However, if a spike in the higher harmonics of the signal is visible only in a RPM band where torsion is known to occur, it could be argued that torsion response was picked up by the pressure sensors. 
The most effective way to analyze the change in the Kulite pressure spectrum with time is with FT waterfall plots. The waterfall plots are constructed through the use of dividing up each channel's pressure signal throughout the sweep into separate revolutions through the use of the once-per-revolution trigger signal. An FT of each of these signals is taken, and the FT is normalized by blade pass frequency. Simply dividing each revolution by the $1 /$ rev signal is effectively square windowing the signal, which can introduce unwanted artifacts into the frequency spectrum, and thus, a Kaiser window was applied to each FT. A Kaiser window allows a high resolution between two frequency components that are separated greatly in amplitude but closely related by frequency. This windowing technique allows for the detection of small components of the 1T signal that are Doppler shifted to a frequency slightly different than blade pass frequency due to individual blade mistuning. It also allows for a cleaner waterfall plot, which resolves features in a smaller frequency resolution. The FT is plotted with frequency along the y-axis, and each successive FT is lined up next to each other, and placed at their respective RPM on the xaxis. This allows a quick glance at several thousand FT plots at an instant, and also allows one to quickly spot trends or changes in the spectrum with time. Figure 5.16 shows a sweep that has been cut into revolutions by the $1 /$ rev signal. Figure 5.17 and Figure 5.18 show the FT of each of these three revolutions that is used to comprise the waterfall plots. 

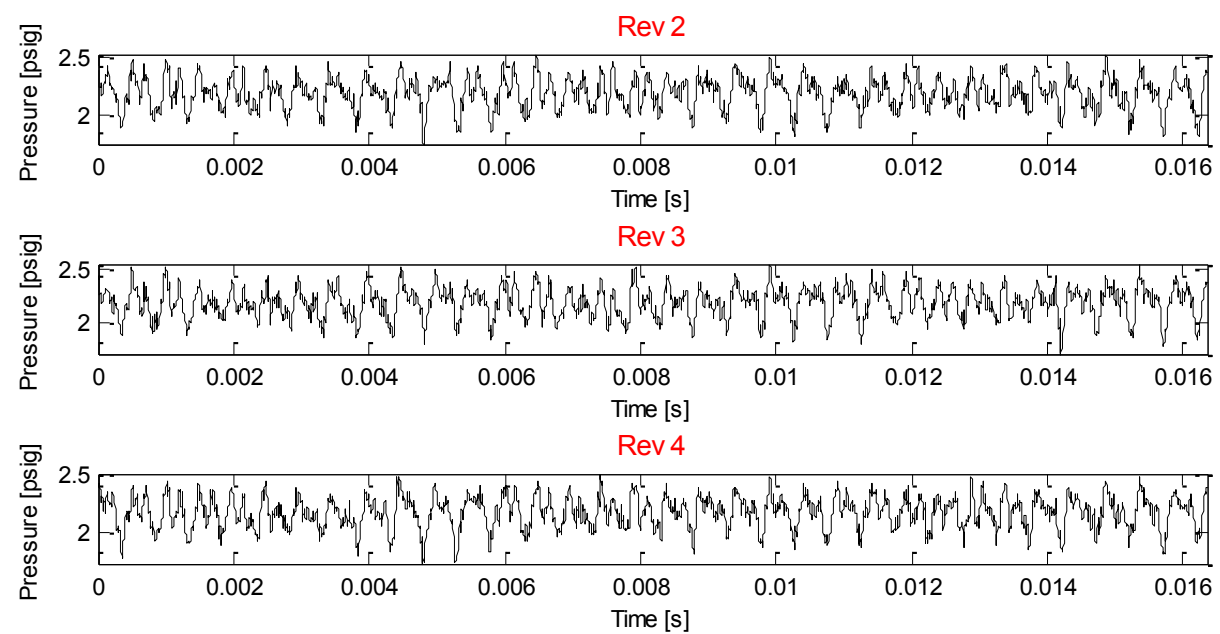

Figure 5.16: Example of successive revolutions of data used for successive FFT generation.

The FT plots are usually normalized by blade pass frequency because it allows the horizontal lines of the plot, corresponding to frequency bands, to be easily analyzed because they are perfectly horizontal, not sloping upward as would be the case as the compressor increases in rotational rate. This allows for easy comparison of the changes in amplitude of a given frequency band as the compressor goes through a sweep. 

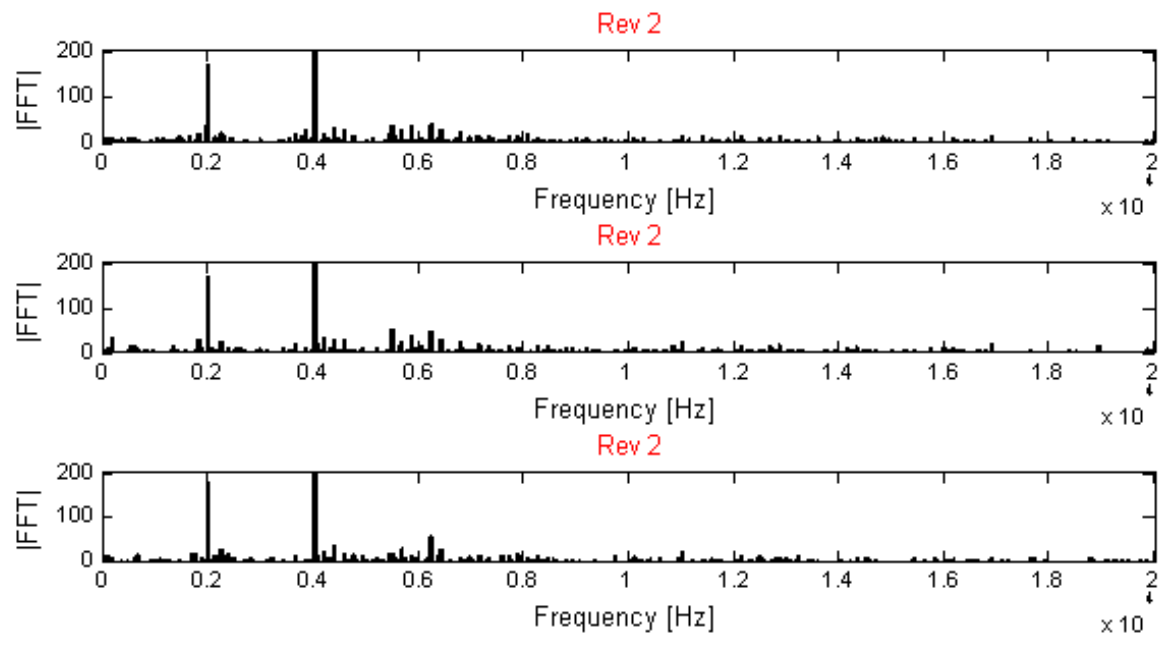

Figure 5.17: FFT generated for each revolution of data in Figure 5.16.
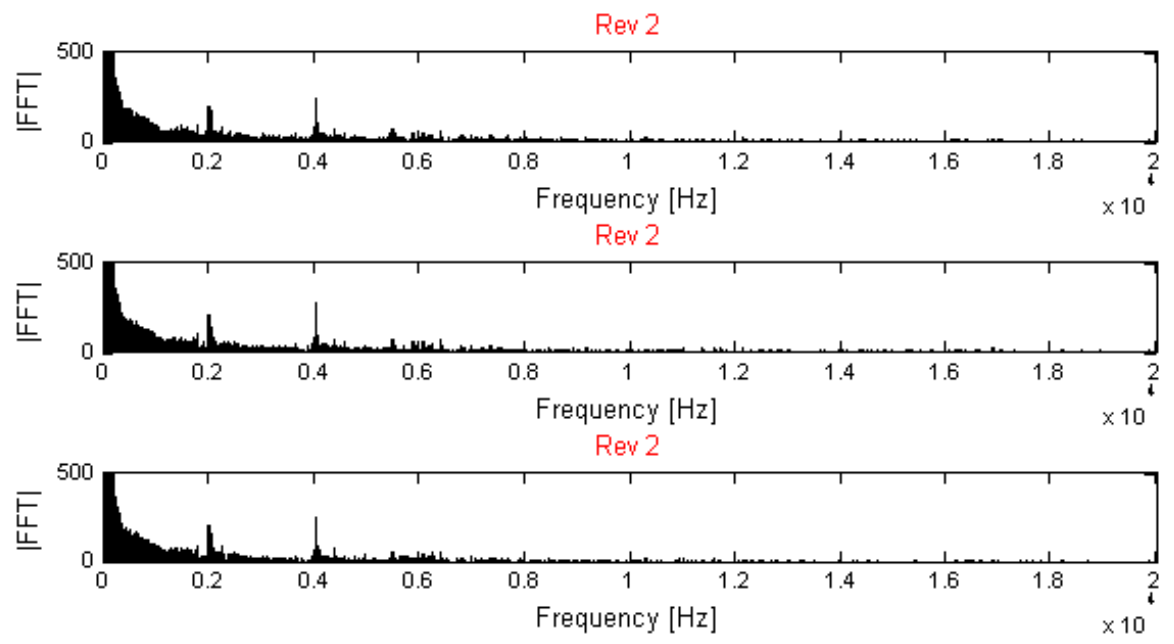

Figure 5.18: Same FFT as in Figure 5.17, but zero padded to length of 30,000 points.

For all stator Kulites, at NL and HL, waterfall plots were generated for several sweeps through resonance. The waterfall plots were all generated in the same fashion, by applying a Kaiser window to the data, zooming in to the $20-120$ freq/rotor rev range, and subtracting out normal BPF related noise at the beginning of the sweep, at 3670 RPM. 
The following figures all seem to have an element in common: the 33/rev frequency component is, for the most part, non-existent, the $66 / \mathrm{rev}$ tends to come and go, and the 99/rev is either non-existent or appears and disappears in conjunction with resonance. There are also other multiples of blade pass frequency that are present, namely responses in the 30/rev, 36/rev, and higher-order harmonics and combinations of harmonics of these responses. This is due to the pressure transducers picking up the destructive and constructive interference of R1 and R3. The manner in which the reflected multiples of these blade pass frequency harmonics are transmitted through the compressor could be indicative of resonance, perhaps even R3 resonance 3705-3805 RPM, but their impact on the Fourier frequency domain is not considered.

As discussed earlier, the frequency bands of interest are those that are multiples of R2 BPF, which is $33 /$ rev, 66/rev and 99/rev, which should show a growth or decay near the same RPM bands that have been attributed to R2 1T response. As Figure 5.19 through Figure 5.22 show that the strength of the response at 99/rev grows down the length of the stator passage. At $40 \%$ chord, the response of the $99 / \mathrm{rev}$ band has grown to over 3 times that of the same frequency response at 10\% chord. Figure 5.23 shows the amplitude of the 99/rev response (taken to be the sum of the frequency band amplitude between 98.5/rev and $99.5 / \mathrm{rev}$ ), and the response grows and decays as a function of compressor RPM, as well as shows how the response becomes greater in amplitude, growing over 3 times as large at $40 \%$ chord as it was at $10 \%$ chord.

Figure 5.19 through Figure 5.22 show specifically the response of the transducers mounted at $80 \%$ span and on the pressure side of the blade which have the largest response to $\mathrm{R} 2$ vibration. There are also several other multiples of $\mathrm{R} 2 \mathrm{BPF}$ that seem to grow further 
down the stator passage, namely 3 x R3 BPF and 66/rev. 3 x R3 BPF does not happen to be a frequency that is predicted to be generated as a result of R2 1T, but there is the possibility that it was created as a result of reflections and transmissions of the torsion response's interaction with neighboring stages.

The 66/rev response is also clearly visible at all chordwise locations, but it is significantly weaker only at $10 \%$ chord. Also, the $66 /$ rev response grows but then seems to level out as RPM is increased, and this could be attributed to the data processing technique - only steady BPF components were subtracted at low RPM's, not at the higher RPMs, before resonance, as shown in Figure 5.24. Therefore, if there is any steady component of the signal that had grown, simply as a result of a higher compressor speed and corresponding TPR, it would be evident in the fact that the frequency band returns to a higher amplitude level than it was at before the sweep. This is not evident in the $99 / \mathrm{rev}$ signal as much as it is in the 66/rev signal.

The 33/rev signal is not apparent in these figures, and this again is a result of the data processing methods. When the steady component of the FFT waterfall plot prior to the sweep was subtracted from the signal, it erased almost all evidence of a 33/rev signal. This does not mean that the component of R2 1T that was Doppler shifted to this frequency range does not exist, it simply means that if it does, it was so small in comparison to the strength of the $33 /$ rev signal that it was subtracted away during data processing. It is not surprising that this is the case - the frequency component of the $1^{\text {st }}$ order harmonic of BPF is orders of magnitude higher than the higher order harmonics, meaning that it would be incredibly difficult to separate any signal attributed to torsion that would amplify the $33 /$ rev signal. Therefore, to detect forced response modes within the vane pressure signal, most 
analysis should turn to the higher frequency harmonics of blade pass frequency where the Doppler shifted forced response frequency components are on the same order of magnitude in amplitude as the ordinary steady BPF components.

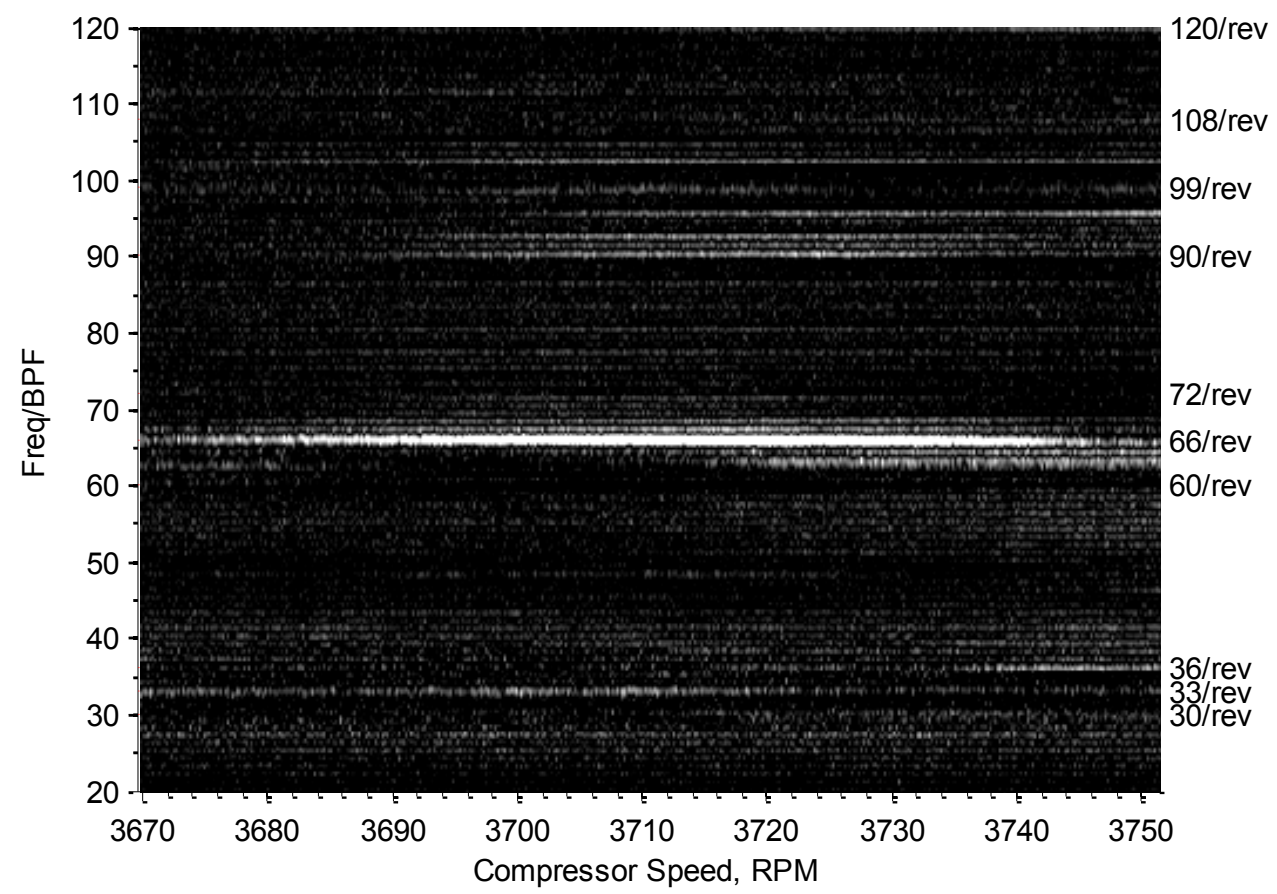

Figure 5.19: FFT Stator Waterfall, Kaiser Window, PS, 80\% Span, 10\% Chord, NL. 


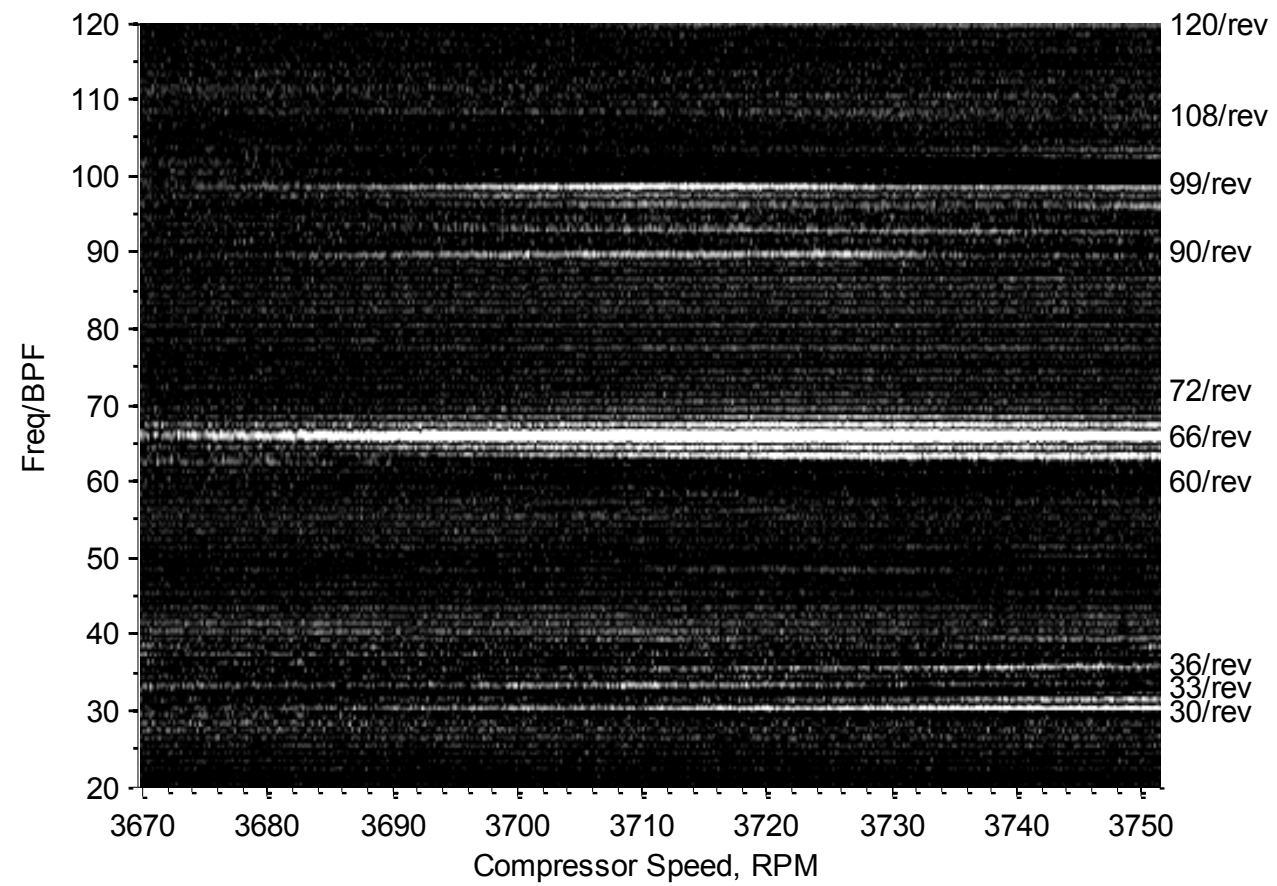

Figure 5.20: FFT Stator Waterfall, Kaiser Window, PS, 80\% Span, 20\% Chord, NL.

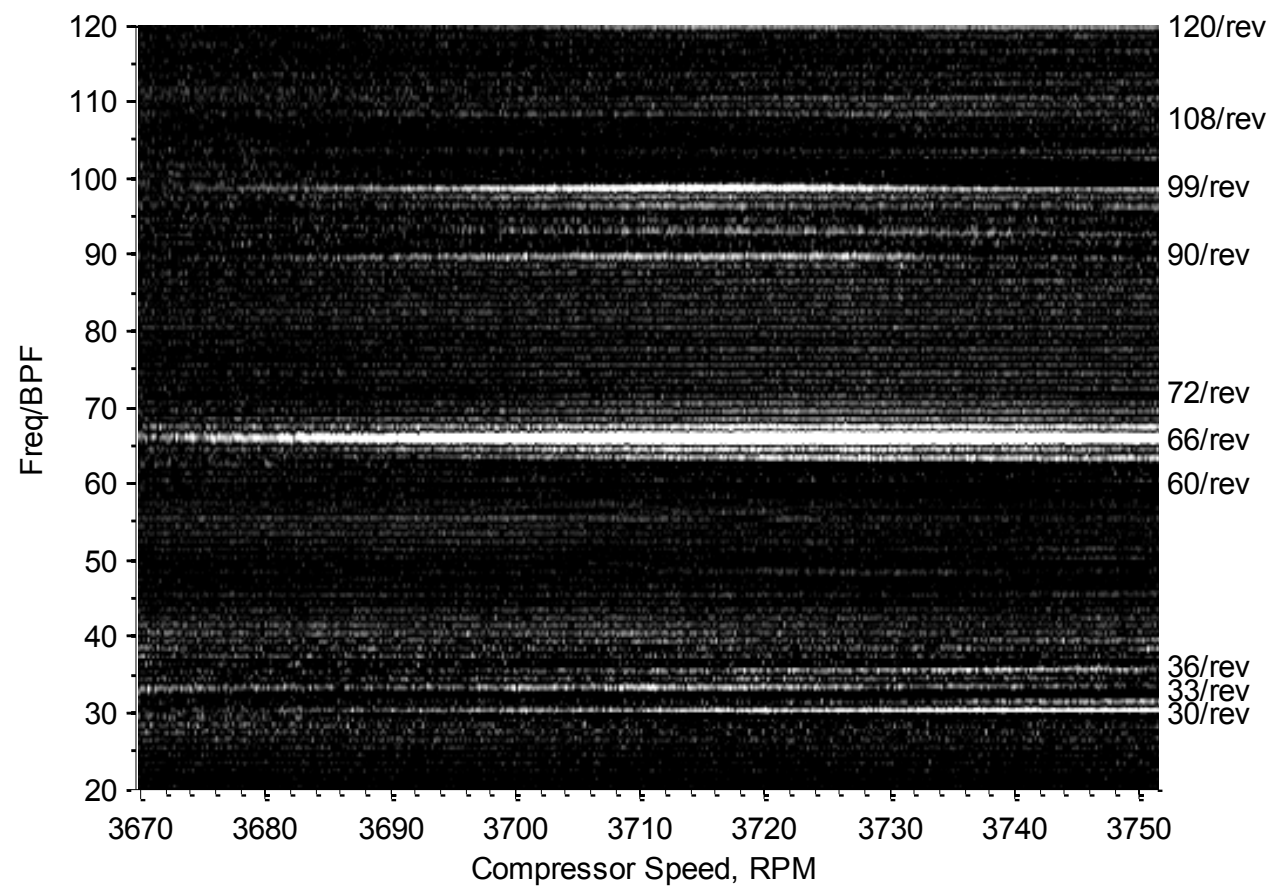

Figure 5.21: FFT Stator Waterfall, Kaiser Window, PS 80\% Span, 30\% Chord, NL. 


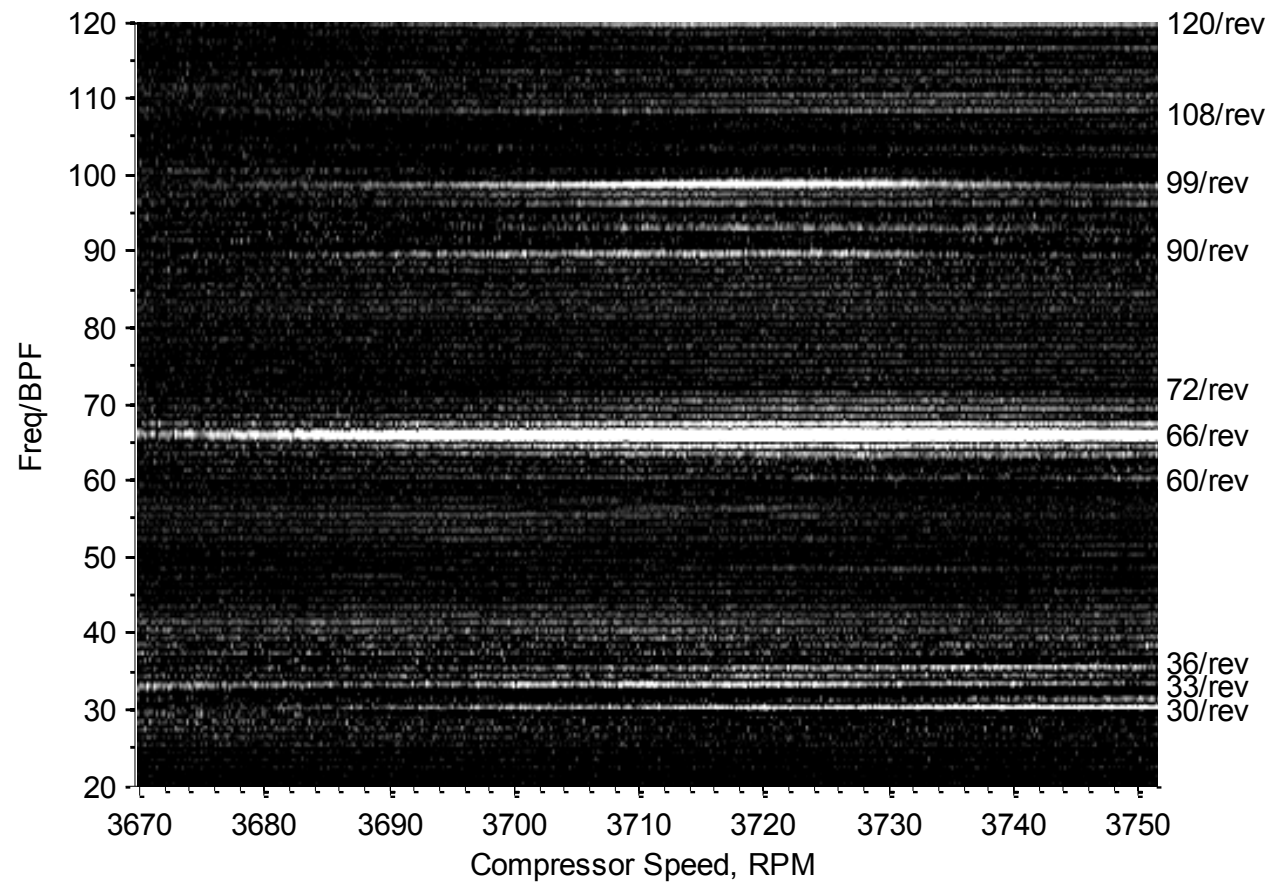

Figure 5.22: FFT Stator Waterfall, Kaiser Window, PS 80\% Span, 40\% Chord, NL.
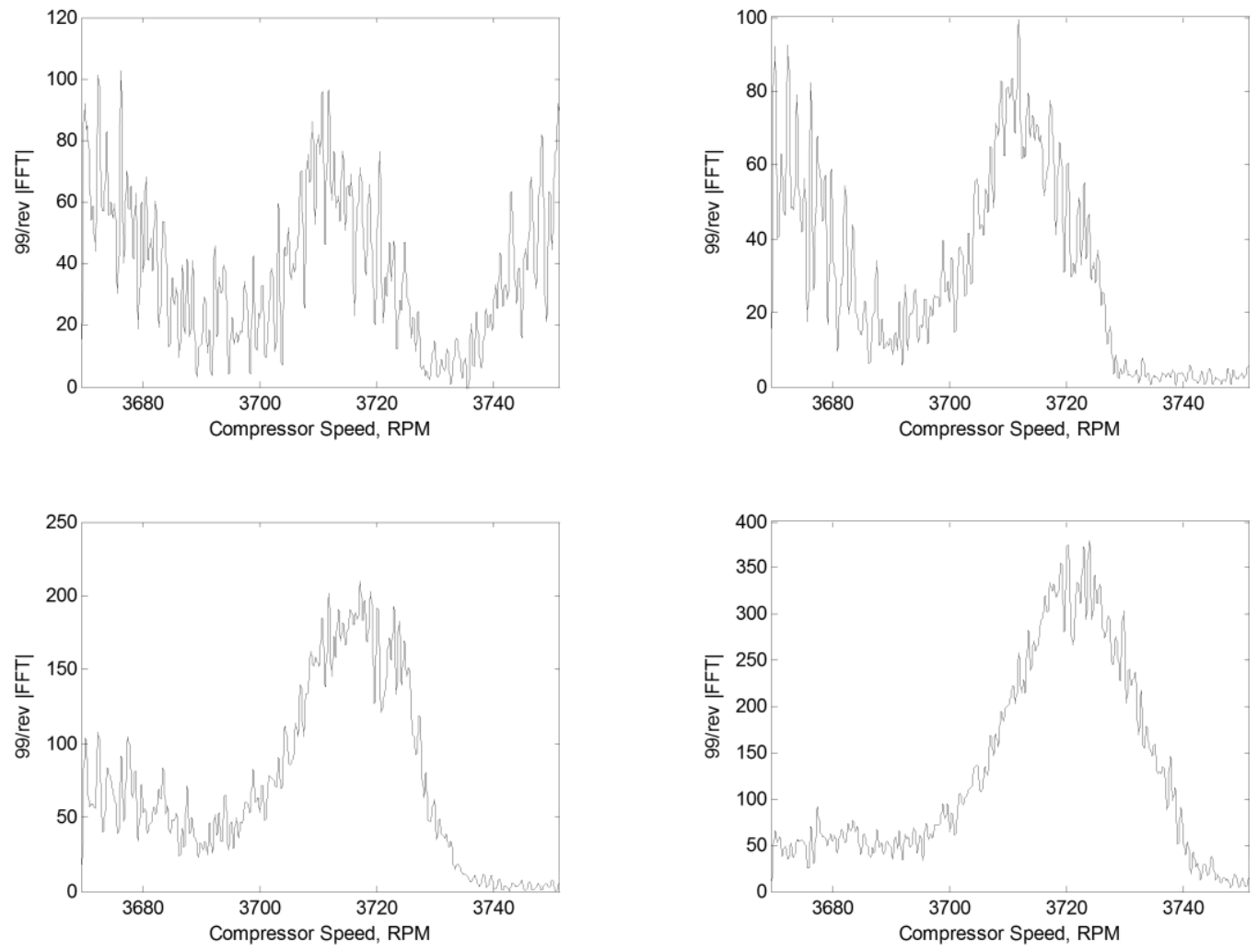

Figure 5.23: 99/rev FFT amplitude, PS 80\% Span, 10\%-40\% Chord, NL. 

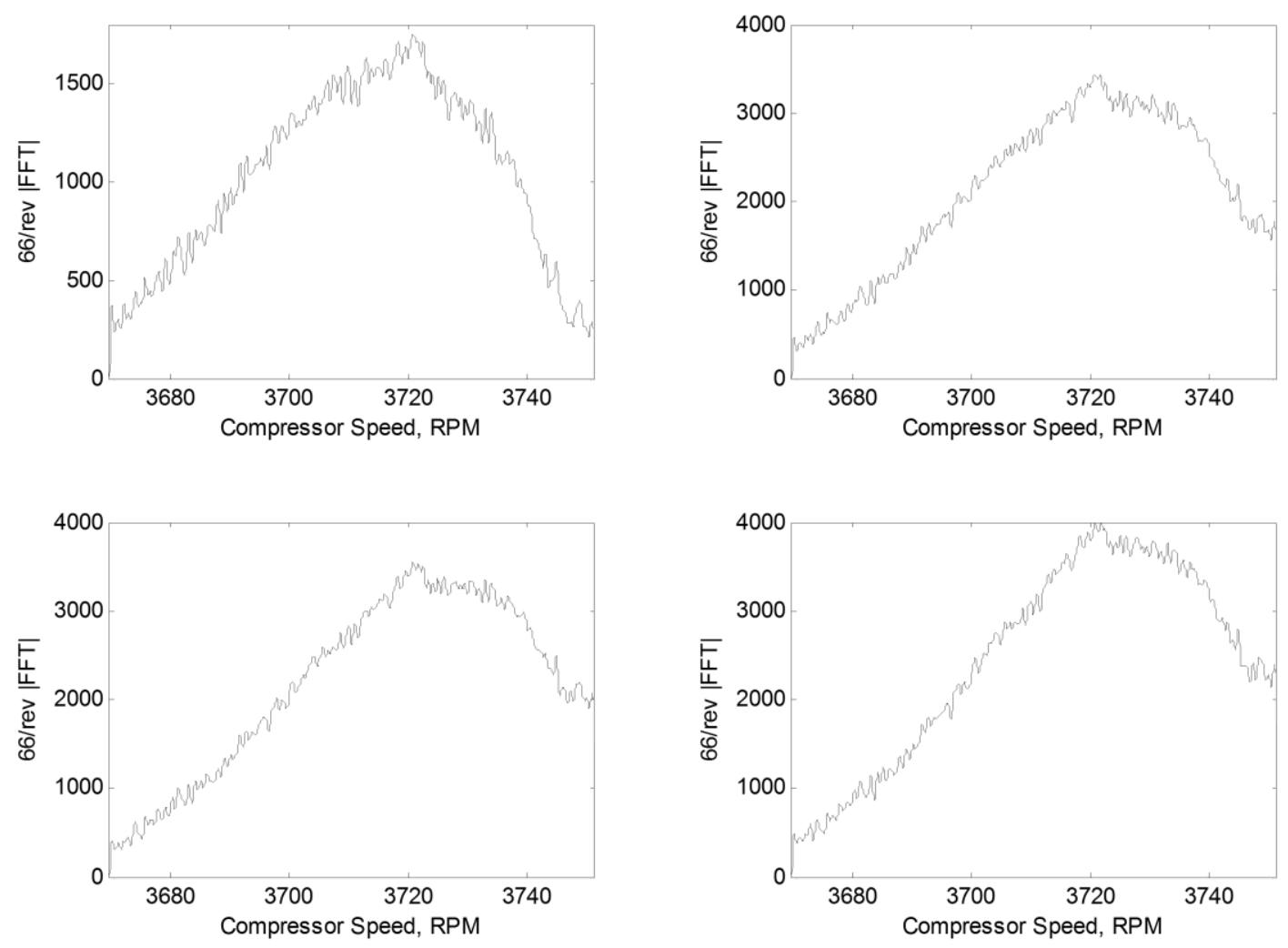

Figure 5.24: 66/rev FFT Amplitude, PS 80\% Span, 10\%-40\% Chord, NL.

Results at high loading show larger amplitudes as well as the torsion response shifted to higher rotational speeds, in Figure 5.25 through Figure 5.29. Particularly in the 99/rev frequency band, Figure 5.29, the amplitudes are both larger than NL cases, as well as shifted far to the right, similar to the $66 /$ rev frequency band in the NL case. The data terminates at approximately 3752 RPM, as this was the limit at which the computer's RAM would fill completely during data acquisition, so it appears as if the response is still decaying by the end of the data run.

The shift of the response to higher values of RPM seems to also be a function of chord position as well, which is interesting and not easily or readily explained. The shift tends to favor higher speeds as chord position is increased, which might be related to some 
amount of attenuation of this particular frequency due to the shape and size of the stator vane passage. Since the spectral components harmonics of BPF, especially the $1^{\text {st }}(33 / \mathrm{rev})$ and $2^{\text {nd }}(66 / \mathrm{rev})$, increase with TPR, part of the increase in the $66 /$ rev component could be due to this feature, therefore creating the illusion that the growth in that harmonic due to R2 $1 \mathrm{~T}$ is shifted to a higher RPM band. The 66/rev amplitude, although not shown separately, is apparent in the waterfall plots to follow a similar trend to the NL cases.

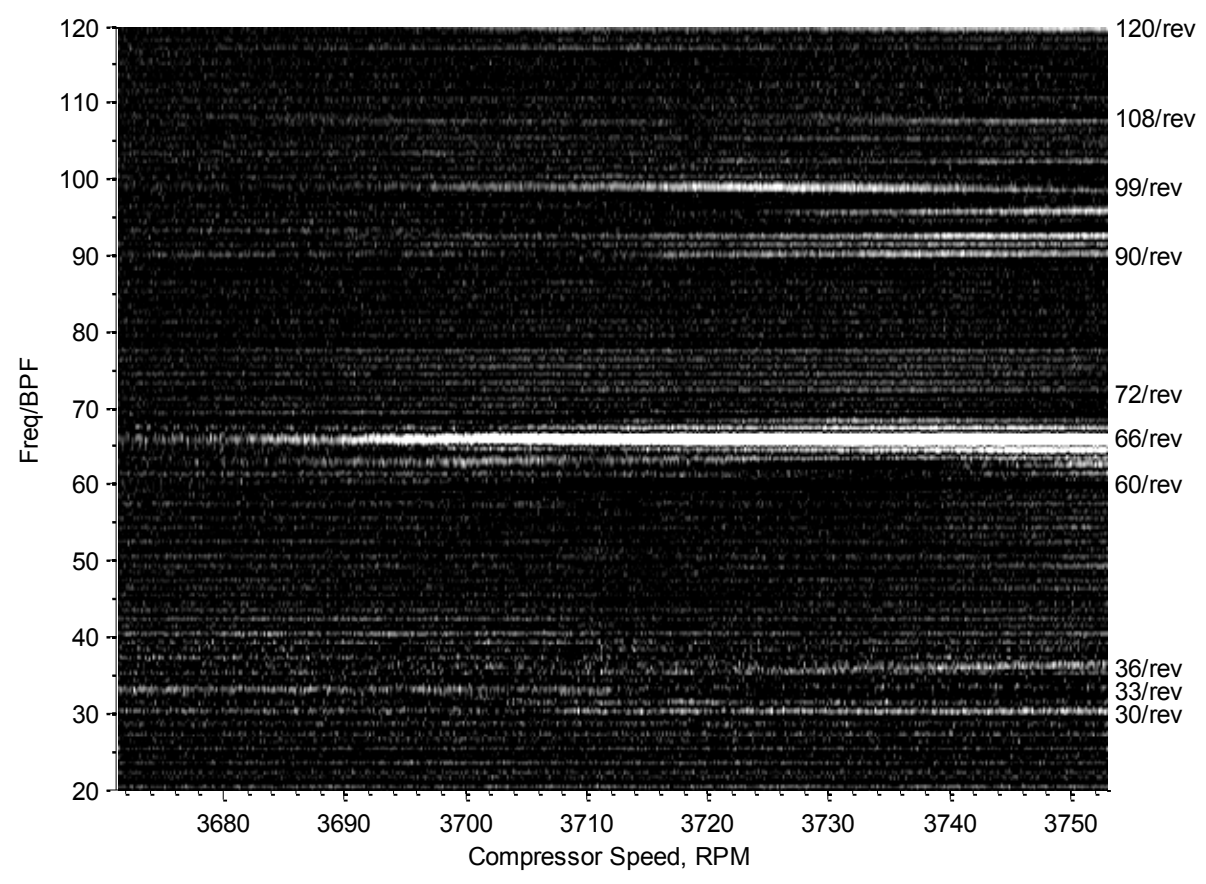

Figure 5.25: FFT Stator Waterfall, Kaiser Window, PS 80\% Span, 10\% Chord, HL. 


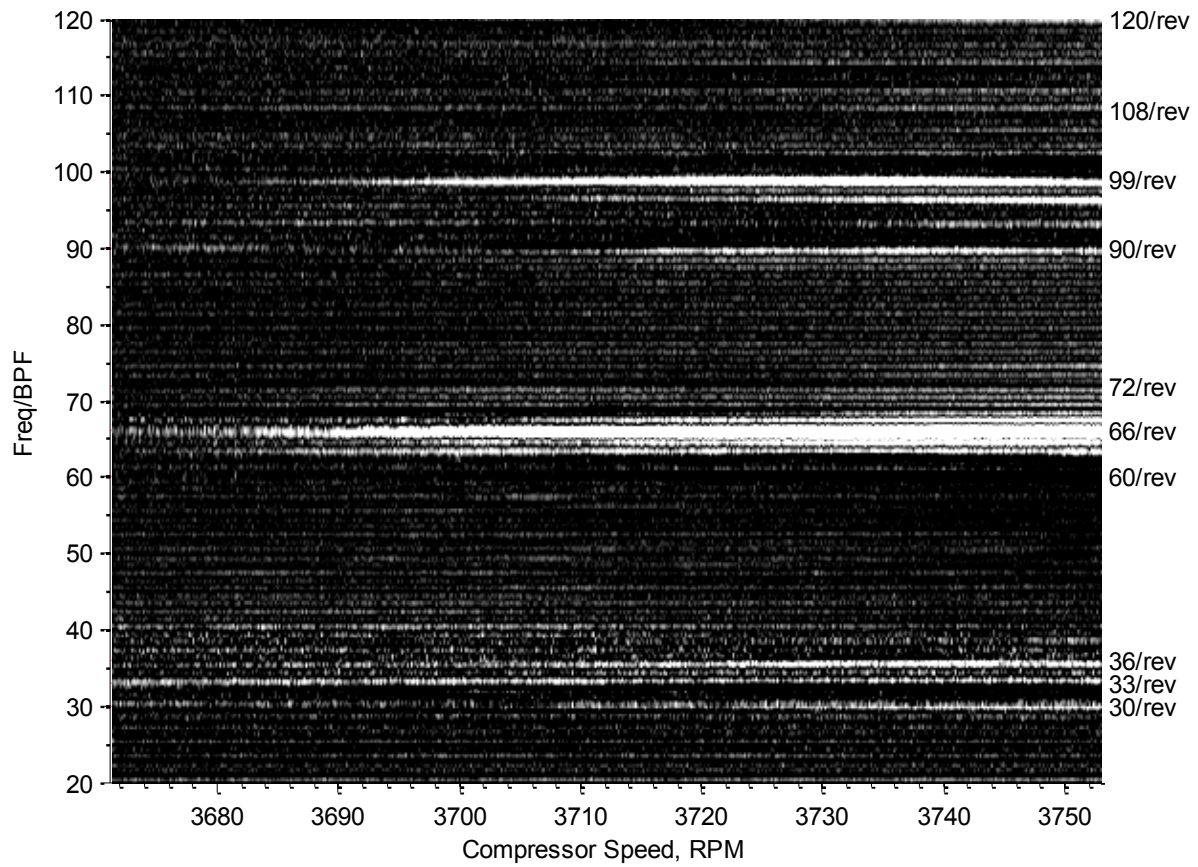

Figure 5.26: FFT Stator Waterfall, Kaiser Window, PS 80\% Span 20\% Chord, HL.

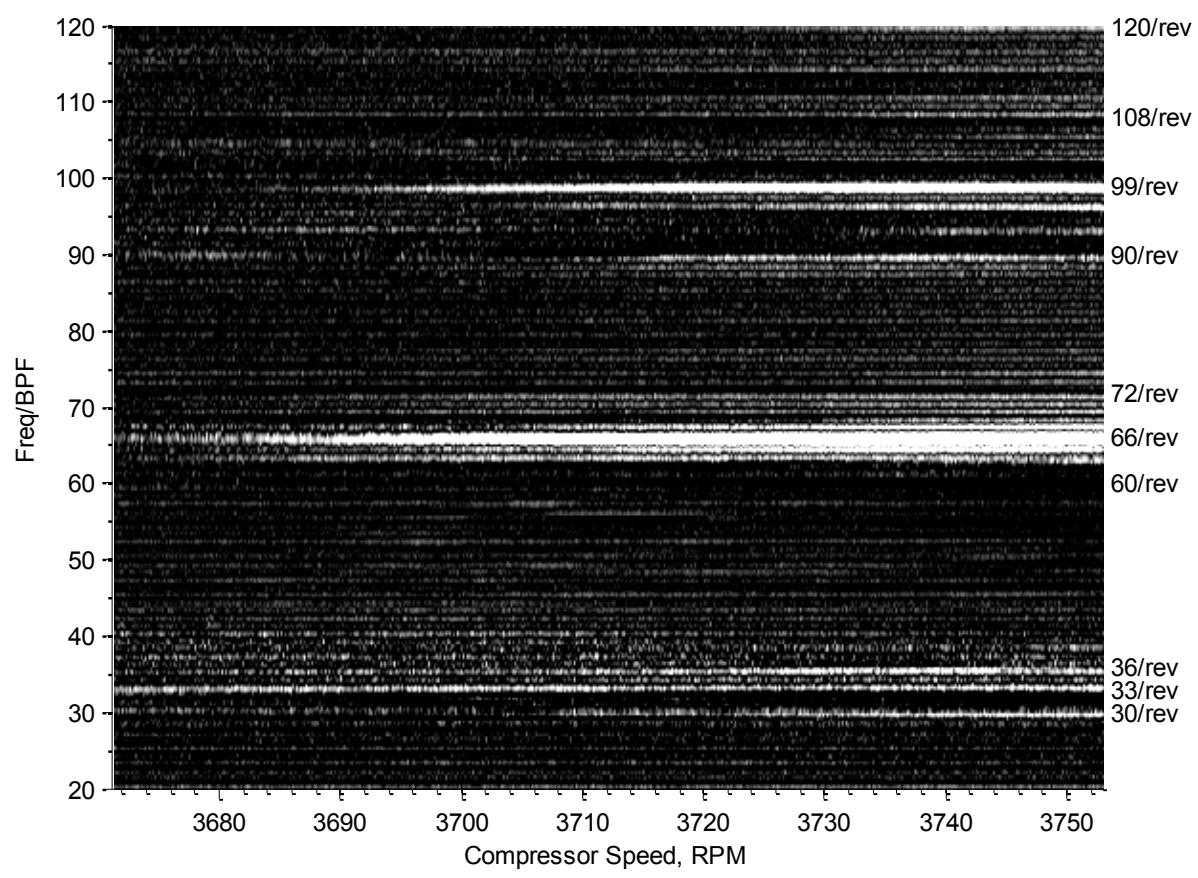

Figure 5.27: FFT Stator Waterfall, Kaiser Window, PS 80\% Span 30\% Chord, HL. 


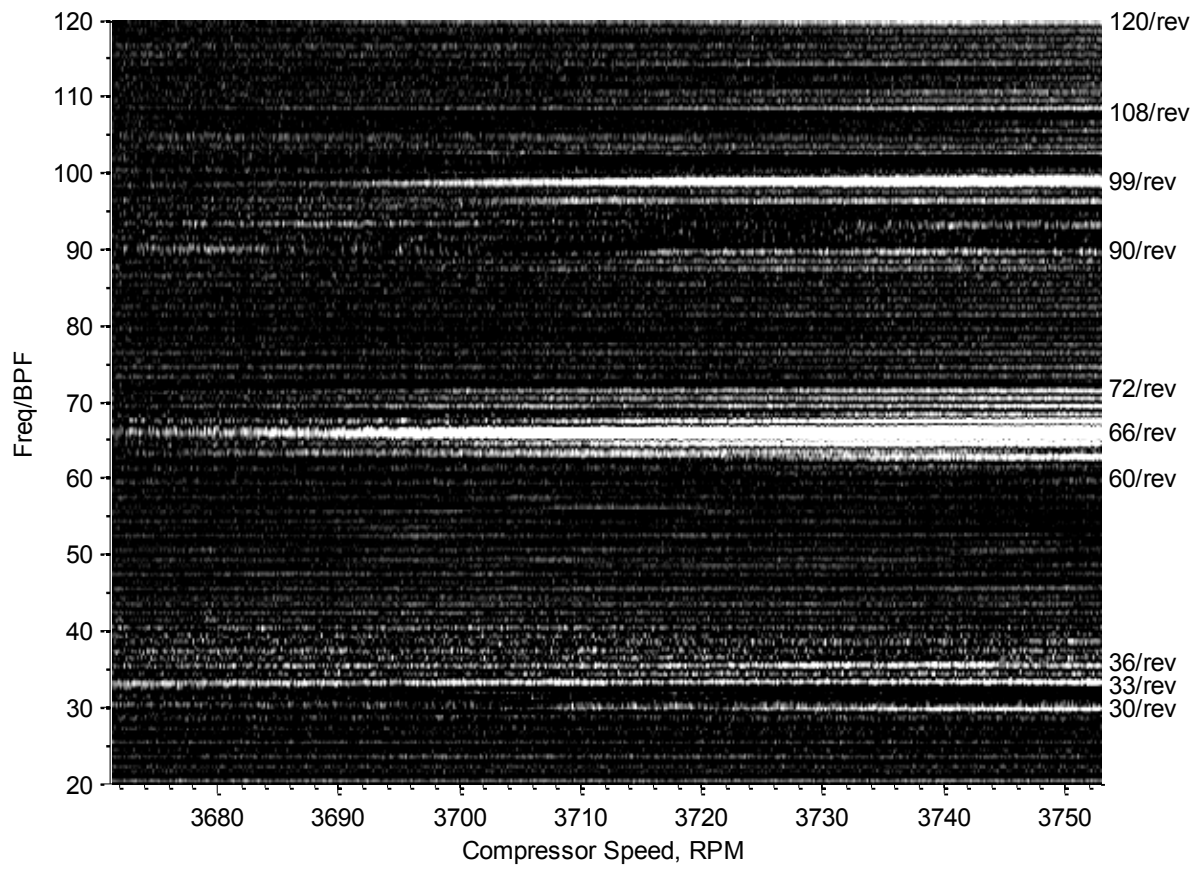

Figure 5.28: FFT Stator Waterfall, Kaiser Window, PS 80\% Span, 40\% Chord, HL.
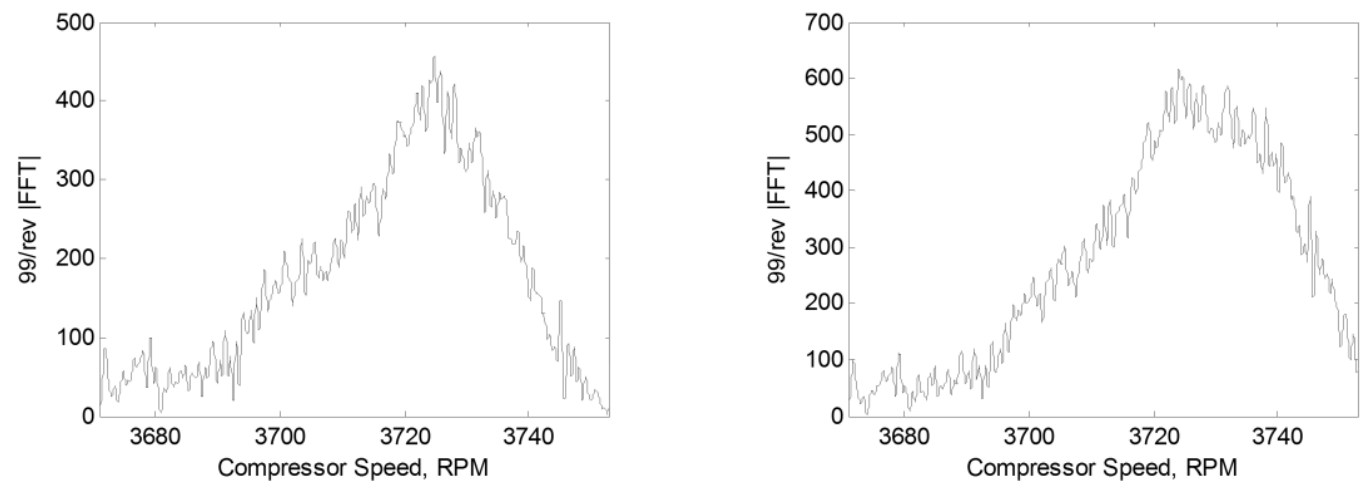

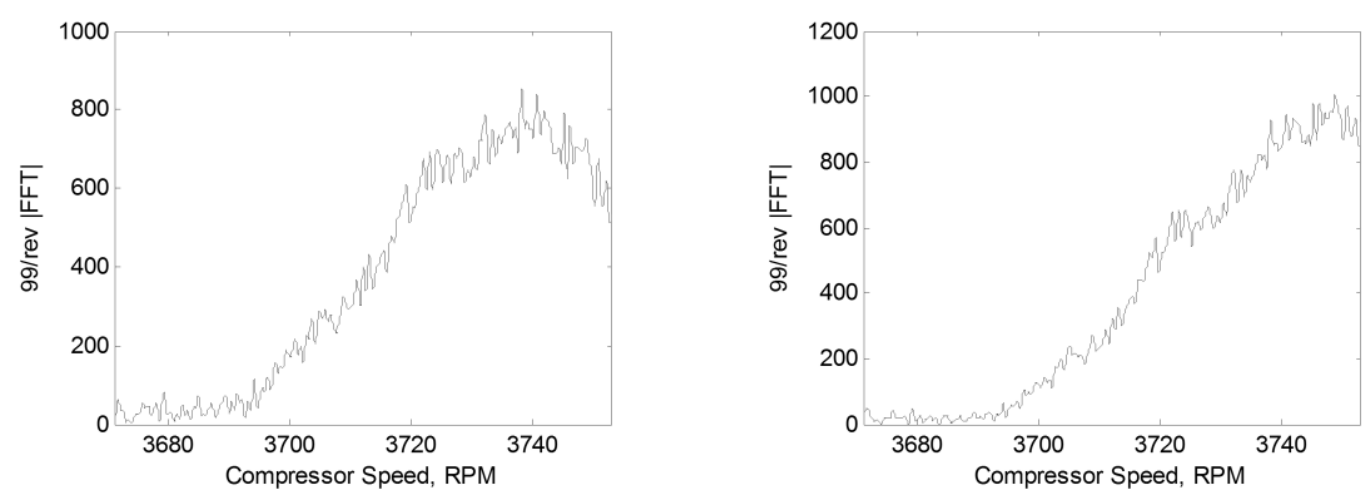

Figure 5.29: 99/rev FFT amplitude, PS 80\% Span, 10\%-40\% Chord, HL.

At $50 \%$ span, the response from only $10 \%$ and $40 \%$ chord are shown on the pressure side to give an idea of the overall response characteristics as a function of chord, shown in Figure 5.30 and Figure 5.31. The trends here are very similar to PS $80 \%$ span at NL, except that the responses are muted significantly. This is important evidence that this is indeed related to R2 1T, because the fact that the responses are lower at mid-span is expected based on the $1 \mathrm{~T}$ mode shape. Figure 5.32 shows the $66 /$ rev frequency grow and decay in a fashion that would be expected from NSMS data, and the 99/rev frequency amplitude growth occurs rapidly and decays, but it is only noticeable at $40 \%$ chord, as shown in Figure 5.33 . 


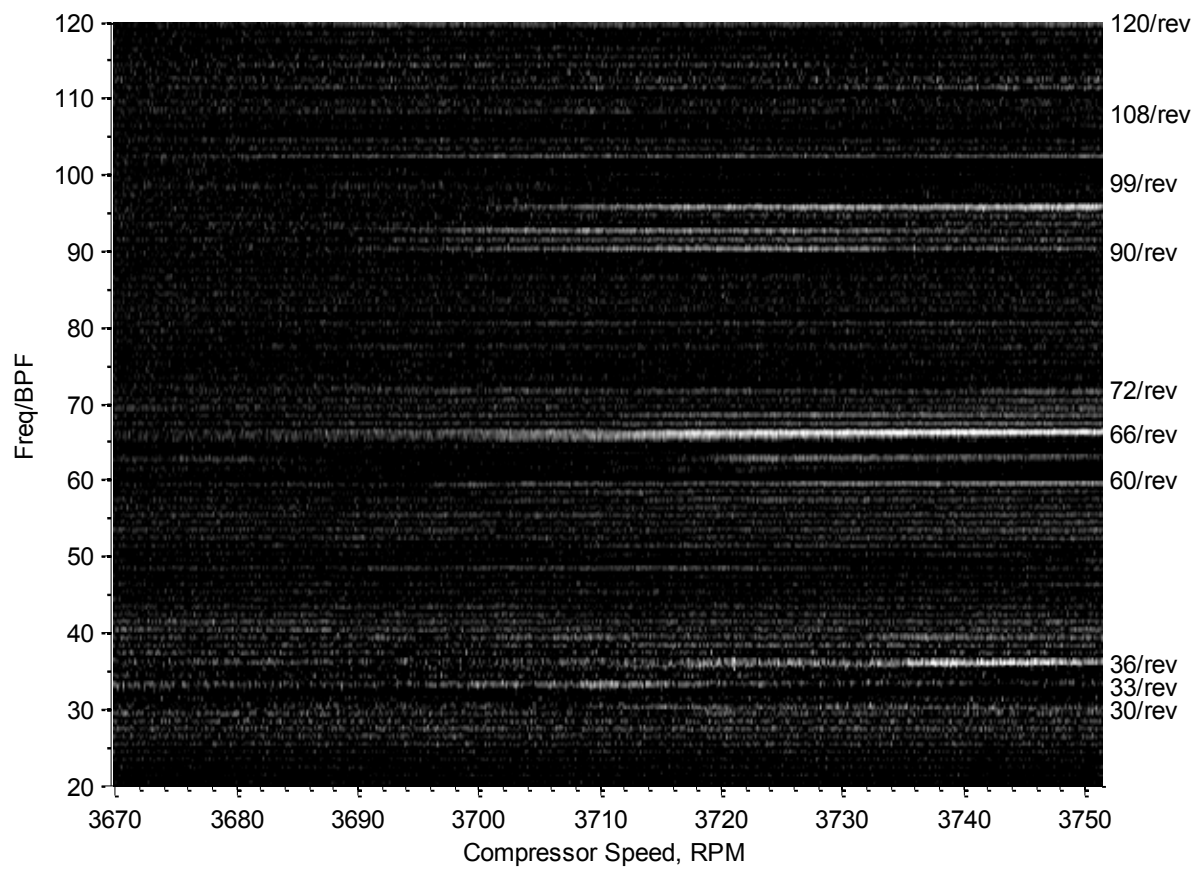

Figure 5.30: FFT Stator Waterfall, Kaiser Window, PS 50\% Span, 10\% Chord, NL.

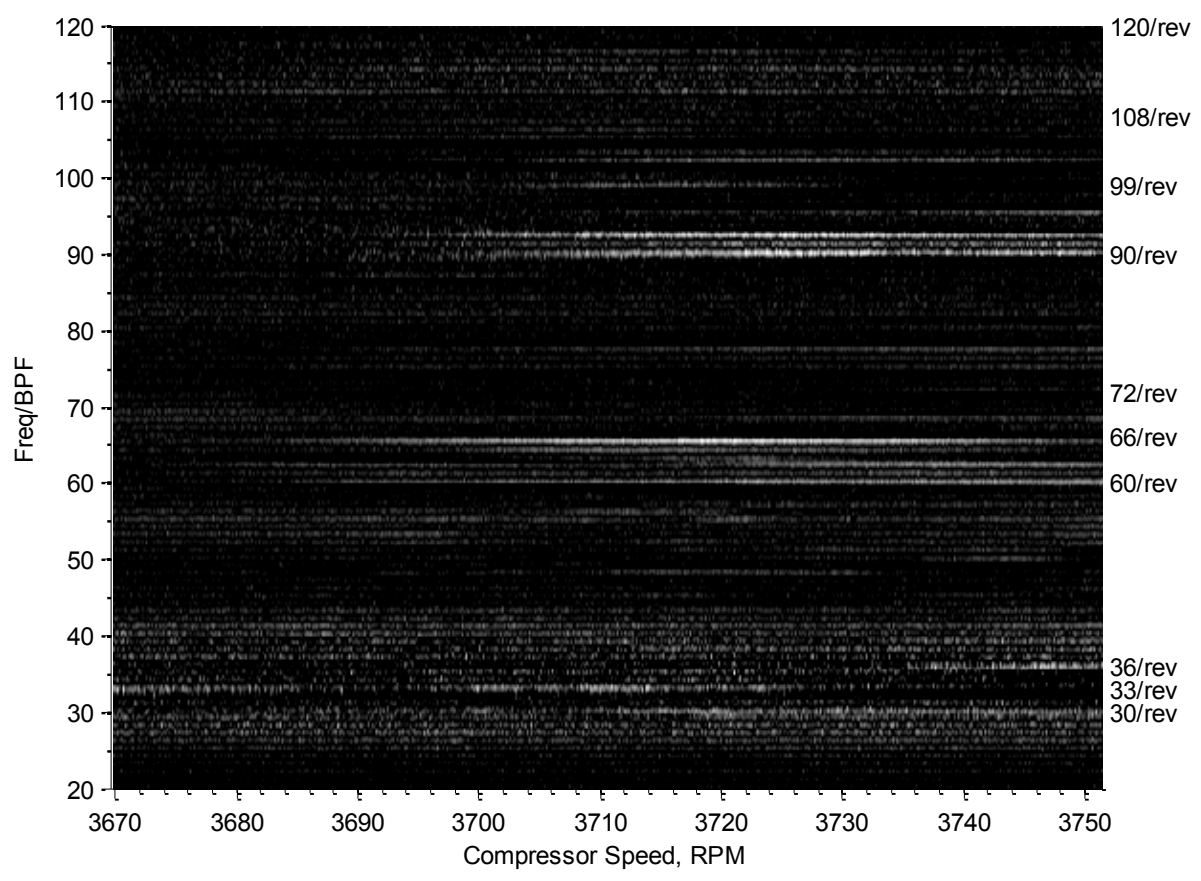

Figure 5.31: FFT Stator Waterfall, Kaiser Window, PS 50\% Span 40\% Chord, NL. 

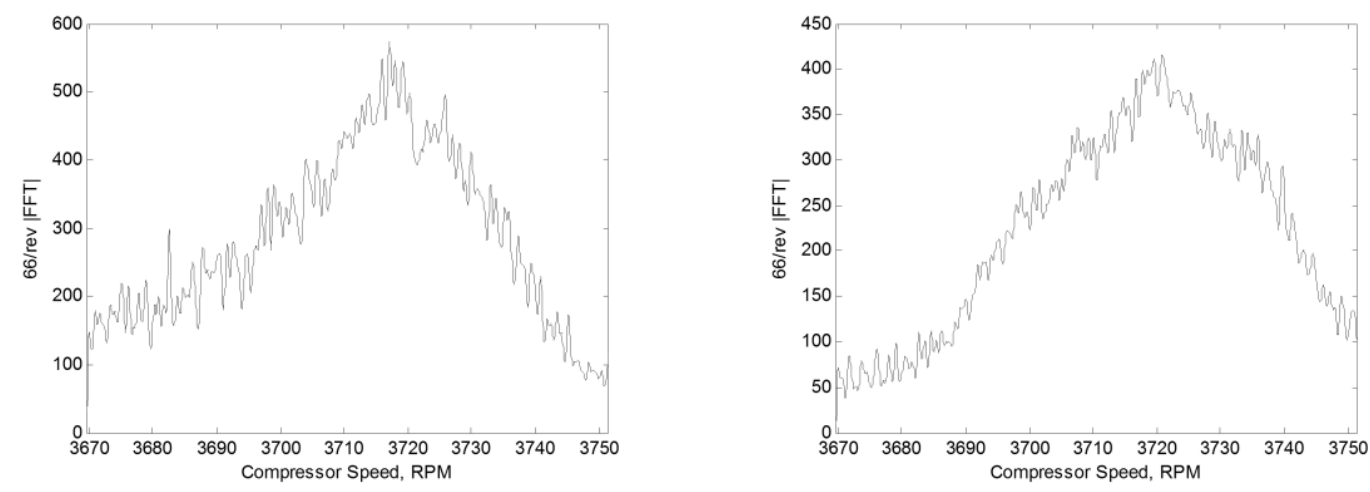

Figure 5.32: 66/rev FFT amplitude, PS 50\% Span, 10\% and 40\% Chord, NL
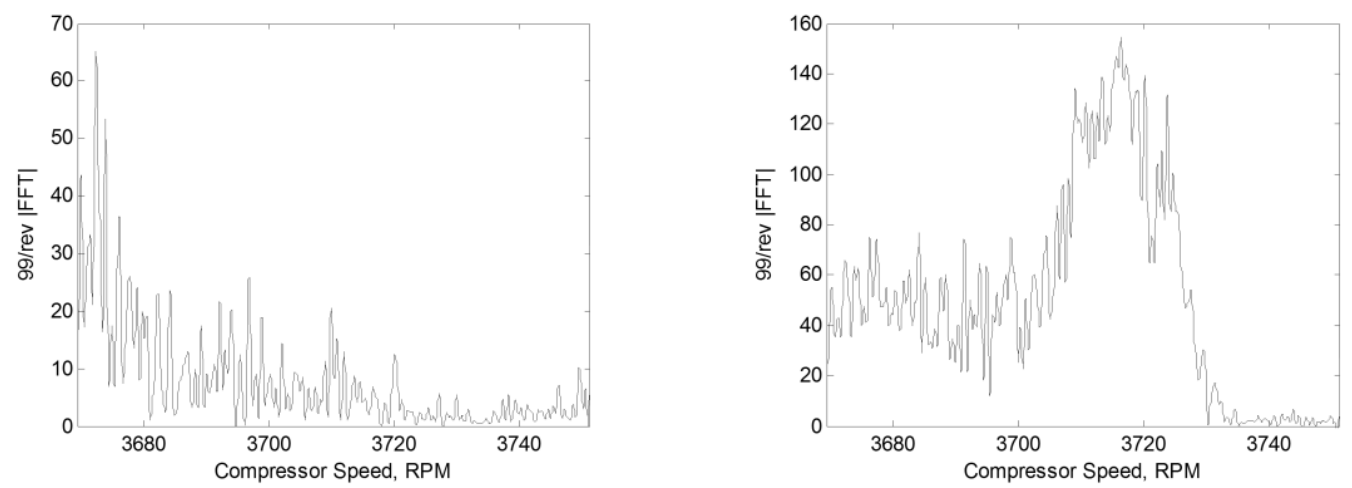

Figure 5.33: 99/rev FFT amplitude, PS 50\% Span, 10\% \& 40\% Chord, NL.

The response of the transducers on the suction side of the blade are also muted in comparison to the responses seen on the pressure side of the blade. However, it is difficult to discern which components of the fluctuating amplitude of the 99/rev and 66/rev are due to torsion. In the FT plots of the 80\% span Kulites, shown in Figure 5.34 and Figure 5.35, the 99/rev amplitude varies greatly as a function of RPM, and grows or decays at RPM values not corresponding to what is seen to be responding RPM bands from NSMS data, shown in Figure 5.36. The only suction side Kulites with a response corresponding directly with RPM values at which R2 responds are those at $10 \%$ and $40 \%$ chord at $50 \%$ span, 
shown in Figure 5.37 and Figure 5.38, respectively. All others seem to have a growth and decay in the 99/rev (Figure 5.39) and 66/rev around 3670-3710 RPM followed by a sharp growth at approximately 3730 RPM. Also, the 66/rev BPF is nearly gone nearer the leading edge of the stator blade and is very apparent deeper within the vane passage.

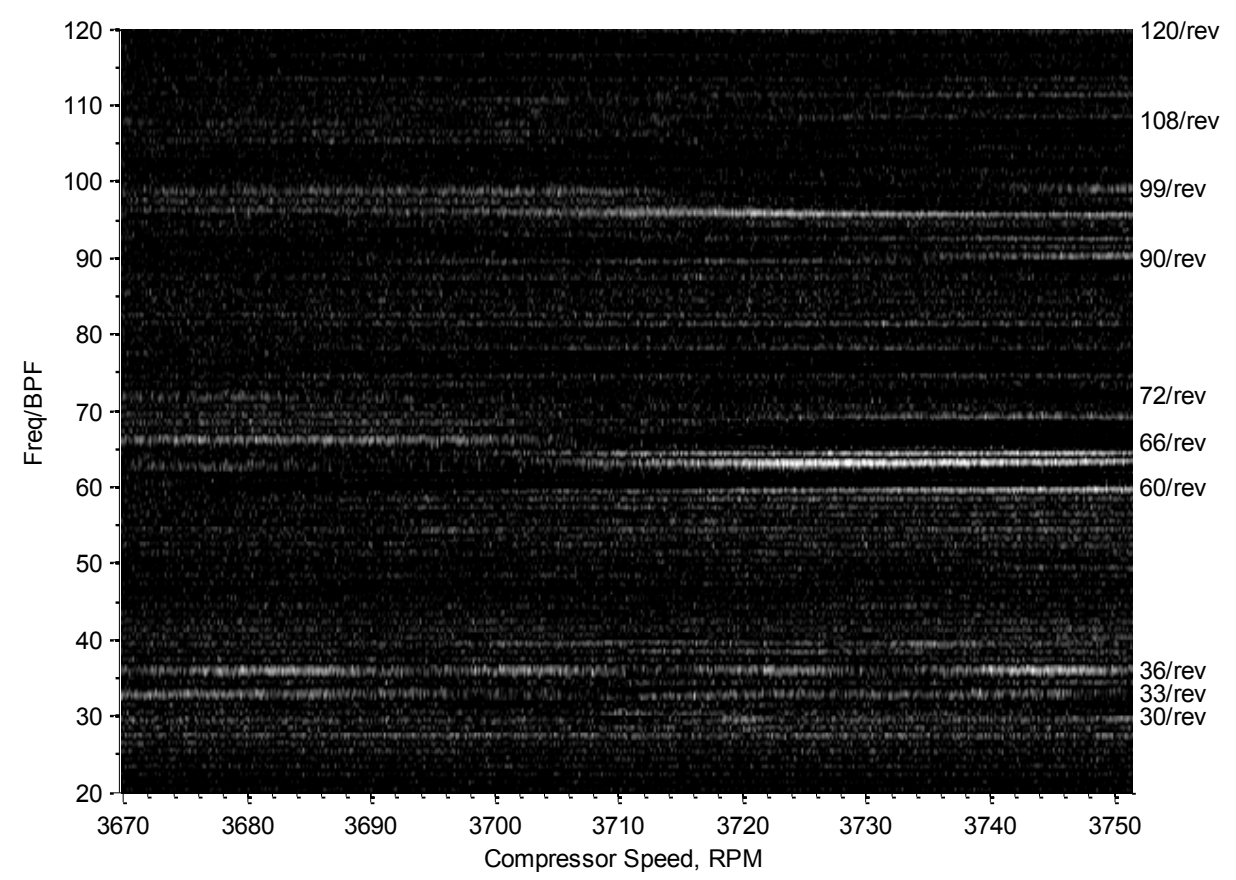

Figure 5.34: FFT Stator Waterfall, Kaiser Window, SS 80\% Span 10\% Chord, NL. 


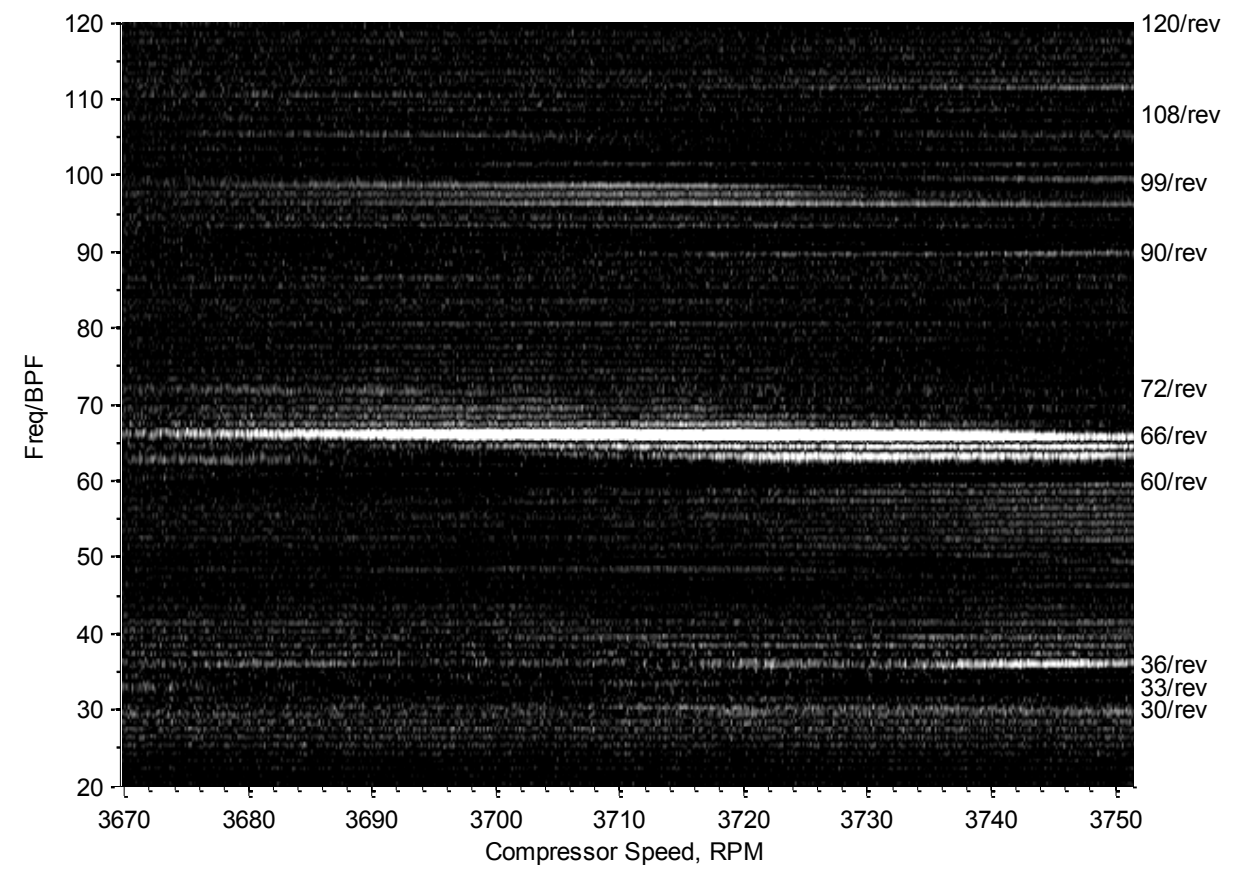

Figure 5.35: FFT Stator Waterfall, Kaiser Window, SS 80\% Span 40\% Chord, NL.
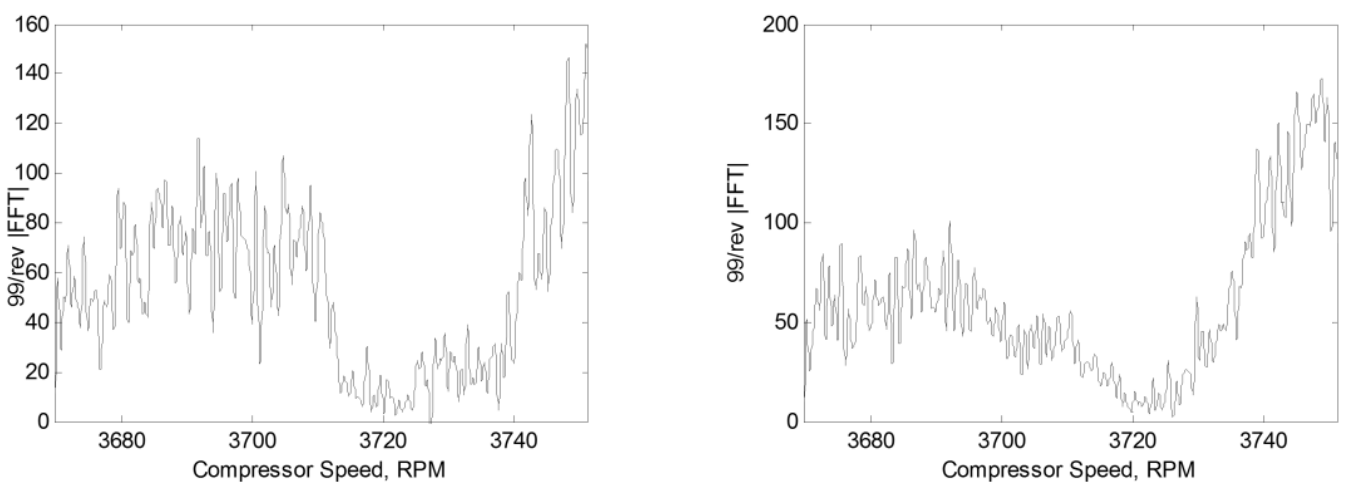

Figure 5.36: 99/rev FFT Amplitude, SS 80\% Span 10\% \& 40\% Chord, NL 


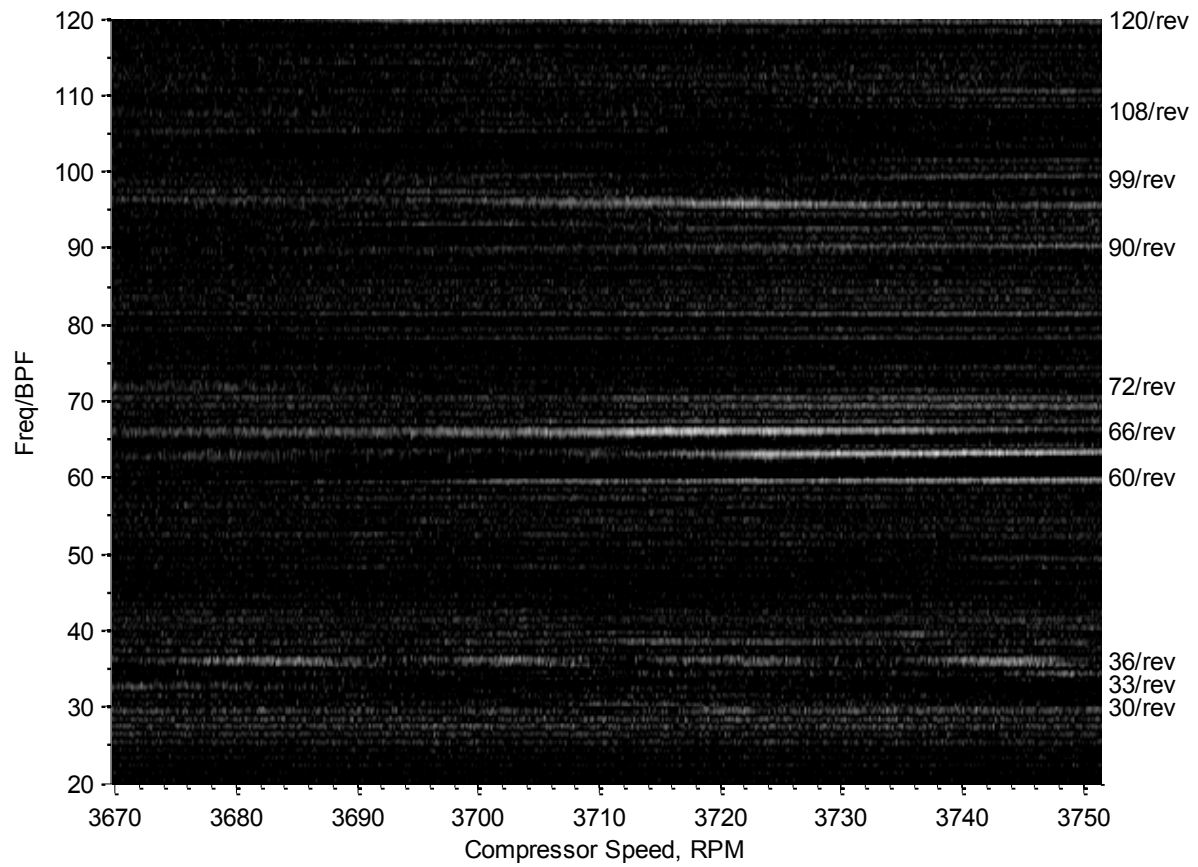

Figure 5.37: Stator Waterfall, Kaiser Window, SS 50\% Span, 10\% Chord, NL.

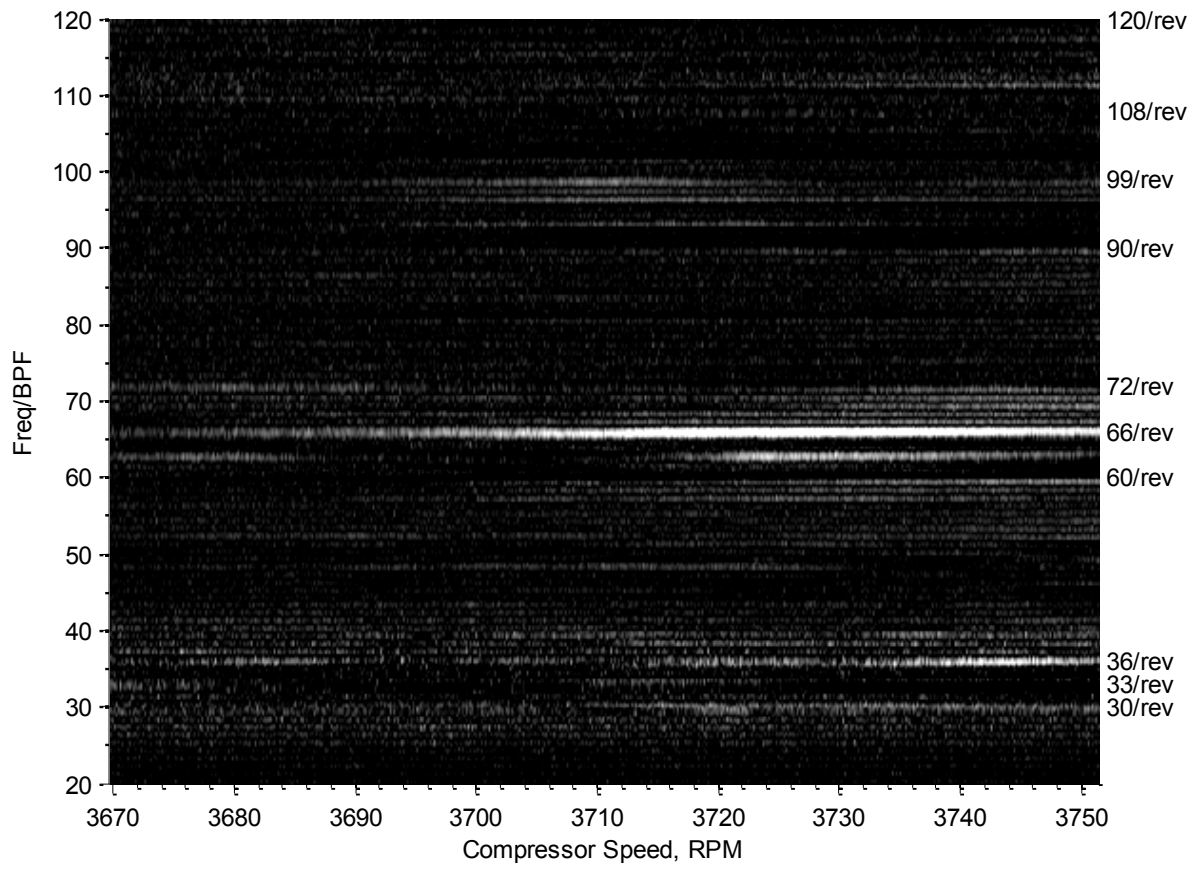

Figure 5.38: FFT Stator Waterfall, Kaiser Window, SS 50\% Span 40\% Chord, NL. 

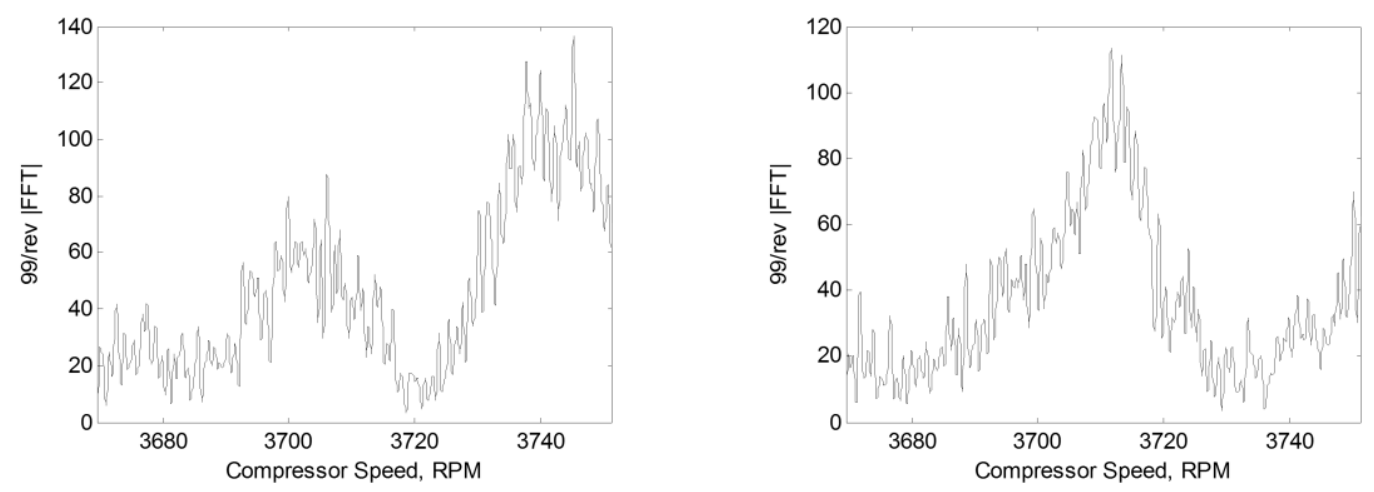

Figure 5.39: 99/rev FFT Amplitude, SS 50Span, 10\% \& 40\% Chord, NL.

The current analysis techniques show that the second and third harmonic of the Doppler shifted R2 vibratory mode was captured by stator Kulites. However, the same FT analysis was conducted with casing-mounted Kulites, and the results were largely inconclusive. There is slight evidence for some change in the 99/rev frequency spectra of a select few sensors, but the change is much smaller in relation to the BPF related spectra, and it is, therefore, more difficult to detect.

\subsubsection{Cross-Correlation Techniques}

The frequency domain analysis shows traces of R2 1T resonance in the Doppler shifted frequency bands in which the forced response would be expected to appear. However, this requires the use of clever subtraction methods that nullify the effects of steady, non-vibrating compressor frequency spectra, which may or may not be an easy assumption to make. Depending on whether or not other stages in the compressor are experiencing resonance near the stage that is being measured, it may be difficult to select an RPM range from which to subtract out steady frequency spectra. If an RPM band very far away from the RPM range in which the rotor responds is used to subtract steady spectra, 
the spectral content could be very different from the steady spectral content present in the compressor speed range of interest and result in an over-subtraction of spectra that would otherwise show a change in amplitude during resonance. The issue of spectral amplitude growth as a function of compressor TPR itself could make it difficult to subtract a useful portion of the steady spectra, either over subtracting or under subtracting, depending on whether or not the subtracted spectra is from a higher or lower TPR, respectively.

To avoid this issue the blade pressure traces are investigated in the time domain. A method of cross correlation has been employed to analyze the changes in the pressure signal of each blade as they pass through resonance in a compressor speed sweep. The same data that were used to generate the Fourier transform waterfall plots were used to calculate trends of cross correlation, cross covariance, and standard deviation as a function of RPM.

Cross correlation and cross covariance are methods of determining the "similarity" or likeness, of two random vectors or signals, denoted by $x$ and $y$ with length $\mathrm{N}$. In MATLAB, there exists two functions, xcorr and xcov, which compute the correlation sequence for two vectors as a function of lag offset, denoted by $m$. By varying the lag offset a correlation sequence of two vectors is generated and is a function of the elementby-element product of each point in the vector.

$$
\hat{R}_{x y}(m)= \begin{cases}\sum_{\substack{n=0 \\ \hat{R}_{y x}^{*}(-m)}}^{N-m-1} x_{n+m} y_{n}^{*}, & m \geq 0 \\ & m<0\end{cases}
$$


This version of cross correlation, however, is not normalized and the values of the product of the two vectors could be any real number, which may or may not be useful to data processing. However, specifically in the case of analyzing the pressure signal from the compressor during a sweep through resonance, the amplitude of the mean level of pressure continually increases due to the increase in TPR. Since cross correlation, in essence, outputs the product of two vectors, the product of a pressure trace taken at the beginning of a sweep compared to a pressure trace at the end of a sweep will have a higher cross correlation value than two waveforms compared with a smaller separation in RPM and therefore TPR. Therefore, the analysis also considers the cross covariance term, which is the product of two vectors but takes into account the mean of each signal, denoted by $\left(\frac{1}{N}\right) \sum_{i=0}^{N-1} x_{i}$ and $\left(\frac{1}{N}\right) \sum_{i=0}^{N-1} y_{i}^{*}$. This is very useful, since it is able to negate the overall rise in correlation due to the rise in TPR and is the preferred method of time-domain analysis of Kulite pressure signals in the compressor.

$$
\begin{aligned}
& c_{x y}(m) \\
& =\left\{\begin{array}{l}
\sum_{\substack{n=0 \\
c_{y x}^{*}(-m)}}^{N-|m|-1}\left(x(n+m)-\left(\frac{1}{N}\right) \sum_{i=0}^{N-1} x_{i}\right)\left(y_{n}^{*}-\left(\frac{1}{N}\right) \sum_{i=0}^{N-1} y_{i}^{*}\right) m \geq 0 \\
m<0
\end{array}\right.
\end{aligned}
$$

The cross covariance terms were not normalized in amplitude to retain extra information that is useful in analyzing the pressure traces, which is the actual magnitude of the covariance. It is interesting to compare the magnitude of the covariance output from one sensor to the next, to see how "similar" the waveforms are from revolution to revolution. It can be used as a measure of noise and cleanliness in each transducer-sensors 
without a clean blade pass $\Delta \mathrm{p}$ tend to have overall lower covariance magnitudes than ones with very clear blade pass events, something very useful in gauging the components that make up the signal recorded by each transducer, since this varies greatly depending on where each transducer is placed within the rig.

To track each individual blade throughout resonance, the $1 /$ rev square wave signal was used to break up each individual revolution of data for a total of approximately 1600 revolutions of data throughout the sweep from 3680-3750 RPM. Each revolution of data was further divided into 33 different waveforms, each one assigned to each blade of rotor 2. It is difficult to decide in the continuous pressure waveform assigned to each revolution where the aerodynamic influence of one particular blade opposed to its neigh another is more prevalent in driving the pressure seen by the sensor. Differences in the circumferential width of the flow field emanating from the blades on R2 from either the suction or pressure side of the blade will lend an indeterminate amount of uncertainty to the analysis, and the method by which each revolution is parsed into individual blades could certainly be improved upon. At the time of the $1 /$ rev pulse in the data, the first blade in the analysis was designated to be blade \#12, according to the NSMS blade numbering scheme. Each time the compressor was de-instrumented and the casing removed, it was important to make sure that the relative clocking of the blisk assembly with respect to the gearbox output shaft spline was consistent throughout the data acquisition process.

The effects of increasing compressor shaft speed throughout the sweep resulted in the length of each vector defining the passage of each individual blade to change by no more than 2 data points, based on the sample rate used for data acquisition. This did not have a significant effect on the cross-correlation or cross-covariance values that were 
determined by comparing each successive blade pass to a blade passage event occurring in a region determined by NSMS data to be outside of the resonance band of R2 (3671 RPM), and this change in vector length was therefore neglected. Additionally, any effects due to the change in deviation of the airstream from $\mathrm{R} 2$ blades as $\mathrm{N}_{\mathrm{m}}$ increased was also neglected, which could change the timing at which the pressure waves from R2 impact the sensors placed on S2. When each revolution was divided into 33 vectors of equal length, there was no difference in how the revolution of data was divided based on sensor location. For example, if the first blade passage vector was determined to consist of the first 50 points of data past the $1 /$ rev signal, and this was assigned to be blade 12 , this was done equally for all sensors, regardless of chord wise (axial) position. Based on the recorded sensor pressure values, it was very difficult to determine the propagation speed of pressure-based torsion response down the passage of the stator. Any attempt to change the way the signal was divided as a function of axial position to account for the speed of propagation would be difficult for a couple of reasons.

Without detailed flow information taken at the time the stator Kulite pressure data was taken, it is difficult to estimate what the exact flow propagation speed is, especially concerning the rate of flow propagation within the stator passage itself. Therefore, the data was processed knowing that there would be some "smearing" of information between each blade - perhaps the pressure wave attributed solely to blade 12 would contain a bit of pressure information from either blade 13 or blade 11 due to assumptions made in splicing up each revolution into individual vectors attributed to individual blade passage events. Therefore, perhaps for this reason alone, this analysis is meant to be more of an investigative, qualitative analysis as opposed to an exact quantitative one, and meant to 
explore the possibility of analyzing blades in a fashion that it seems has not been done previously in turbomachinery for forced response problems. Figure 5.40 shows the relative position of the stator section to the Kulite window block, outlined in grey, showing the stator section mounted approximately two rotor blade passages clockwise, forward facing aft. The flow direction in the figure is from right to left, rotor rotation top to bottom.

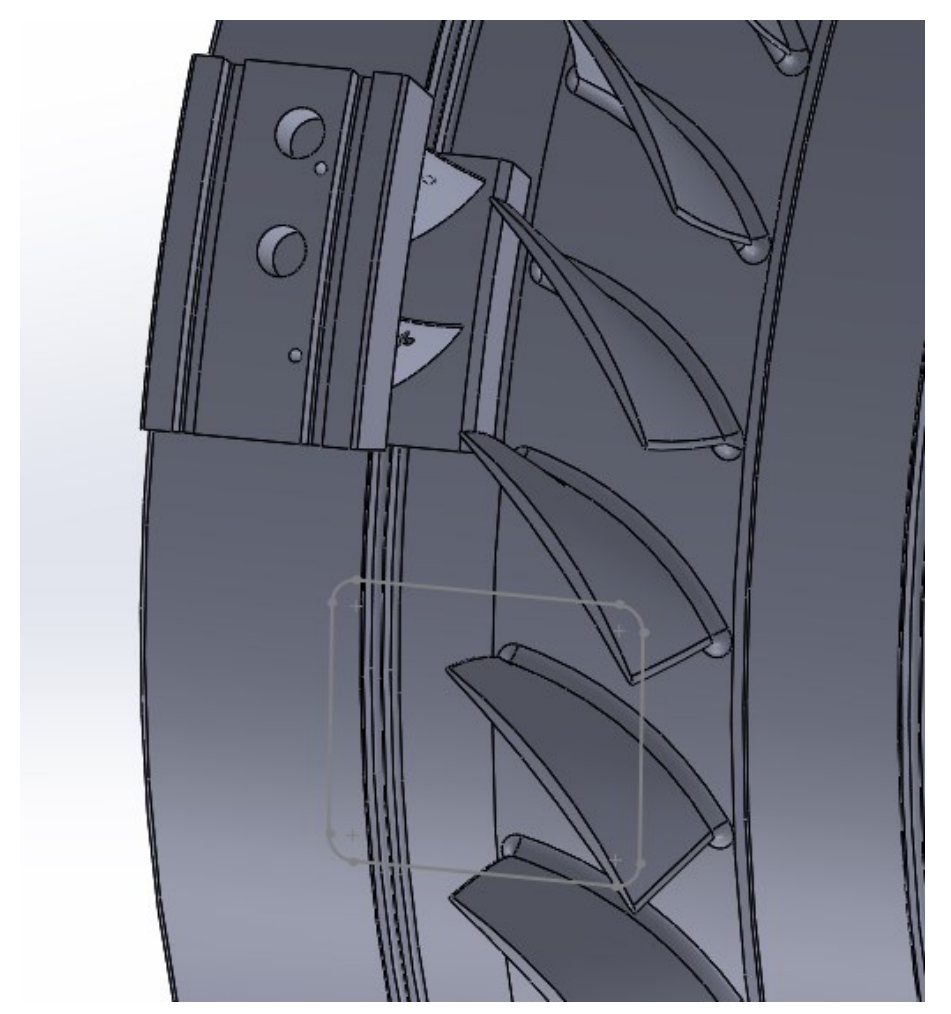

Figure 5.40: Relative position of instrumented stator section to window Kulite measurements.

After dividing each revolution of data into individual blade passage events for each of the 15 transducers on the stator passage, a vector for cross correlation, cross covariance, and standard deviation are created throughout the sweep through resonance by comparing each individual blade passage event at each sensor for each successive revolution to the 
blade passage event within the $1^{\text {st }}$ full revolution of data for the respective sensor. The $1^{\text {st }}$ full revolution of data occurs near 3670 RPM, outside the range of R2 1T for all blades, as determined by NSMS data. The built-in MATLAB functions xcorr and xcov, are used to compare the passage events to one another to see how the "similarity" or the correlation/covariance of the blade passage event changes throughout the sweep. Another function, called mystdev, was created to calculate the sum of the standard deviation element-by-element of both vectors being compared and returns a value, shown in Eqn. $5-15$, where $N$ is the total number of points in each vector, and $x_{i}$ and $y_{i}$ are the $i$-th elements of each input vector. If the vectors are of unequal length, the shorter vector is zero-padded, in a similar fashion to the previously discussed built-in MATLAB functions, $x \operatorname{corr}$ and $x \operatorname{cov}$.

$$
\sigma=\sqrt{\frac{1}{N} \sum_{i=1}^{N}\left(x_{i}-y_{i}\right)^{2}}
$$

It is expected that the blade passage events would change shape enough during resonance that each measure of similarity to the original blade passage event would drop, either from a change in the overall pressure wave shape or a drastic change in blade time of arrival. The revolution-to-revolution variability of each measure of similitude is very large, however, it is evident that there are general trends to the data throughout the sweep that overcome the large amount of "noise" in the data. Cross correlation, cross covariance, and standard deviation are all non-dimensional values, and therefore the magnitude of each calculated value is not as important as the relative change in the value as a function of RPM. 
Each of the similitude vectors are also compared to individual blade NSMS data taken from an acceleration sweep (same sweep direction stator Kulite data was acquired).

There are a total of 2,970 plots generated for similitude analysis due to the possible combinations between two loadings, 15 sensors, 3 measures of similitude, and 33 blades. Therefore, it is very important to narrow down the data that are important to validate this analysis method. In particular, a pressure side, $80 \%$ span, HL case dataset was chosen to display a majority of the data. Pressure side was chosen because these sensors seem to display the largest correlation with NSMS data according to waterfall plots, and it would be expected that the pressure side would experience the changes in pressure more directly than the suction side of the blade. The $80 \%$ span location was chosen because this span location is most subject to changes in flow angle due to the fact that the $1 \mathrm{~T}$ shape produces a trailing edge deflection amplitude that is a positive function of span height. Lastly, high loading was chosen because this produces the largest deflection amplitudes in $1 \mathrm{~T}$ for all R2 blades.

Additionally, it can be argued that only the cross covariance method is useful for investigating R2 1T effects. Standard deviation and cross correlation suffer from a continual upward slope because it is apparent that these measures are strongly a function of rotational speed. This is primarily due to the increase in TPR - both mathematical methods in determining similitude are primarily a function of the amplitude of the pressure wave. As TPR grows, so does the amplitude of each successive pressure wave coming from R2. Because of this slope, it is difficult to determine whether or not changes in slope are due to increases in TPR or from a change in similitude due to 1T. However, it is evident that there may be some effects of rotor vibration that are changing the slope of these lines, 
because there exists a cross covariance measure, a method independent of the change in TPR, it was used for all similitude measurements. Figure 5.41 shows the upward slope in standard deviation for blade 26, the highest responder according to NSMS data. Although there is an apparent discontinuity in slope at approximately 3705 RPM, the slope makes it a bit more difficult to determine whether or not this would be the result of torsion response. Figure 5.42 shows the same blade as measured from the same transducer, but using cross correlation, showing no noticeable trends with a continually increasing slope.

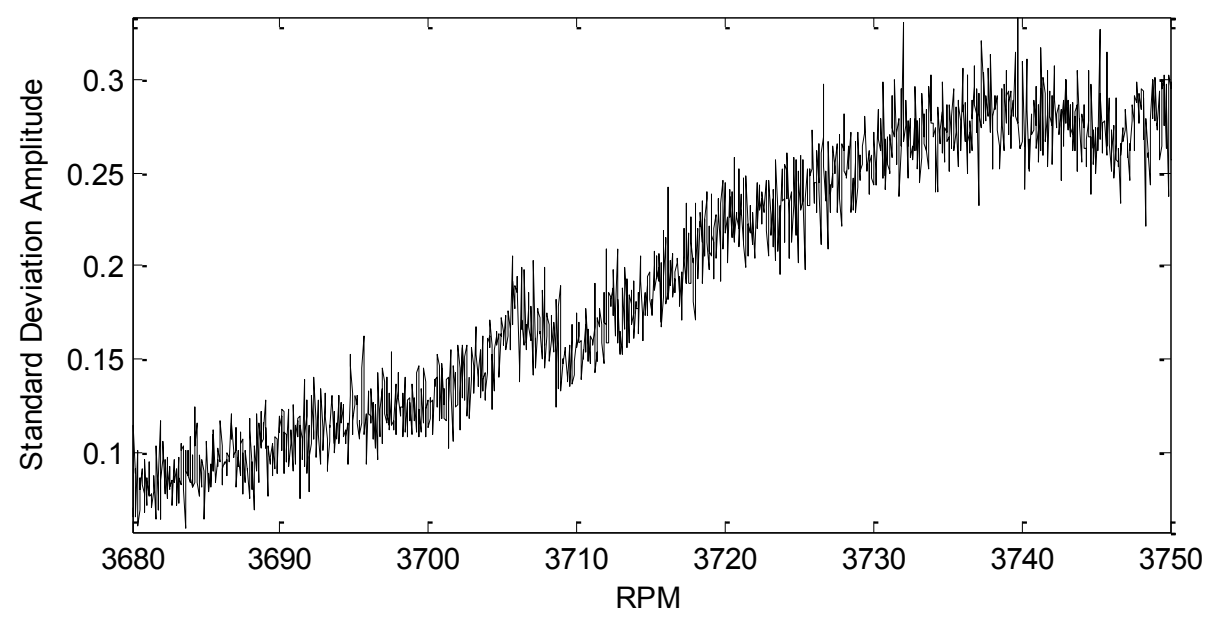

Figure 5.41: Standard Deviation, Blade 26, PS 80\% Span 20\% Chord, HL.

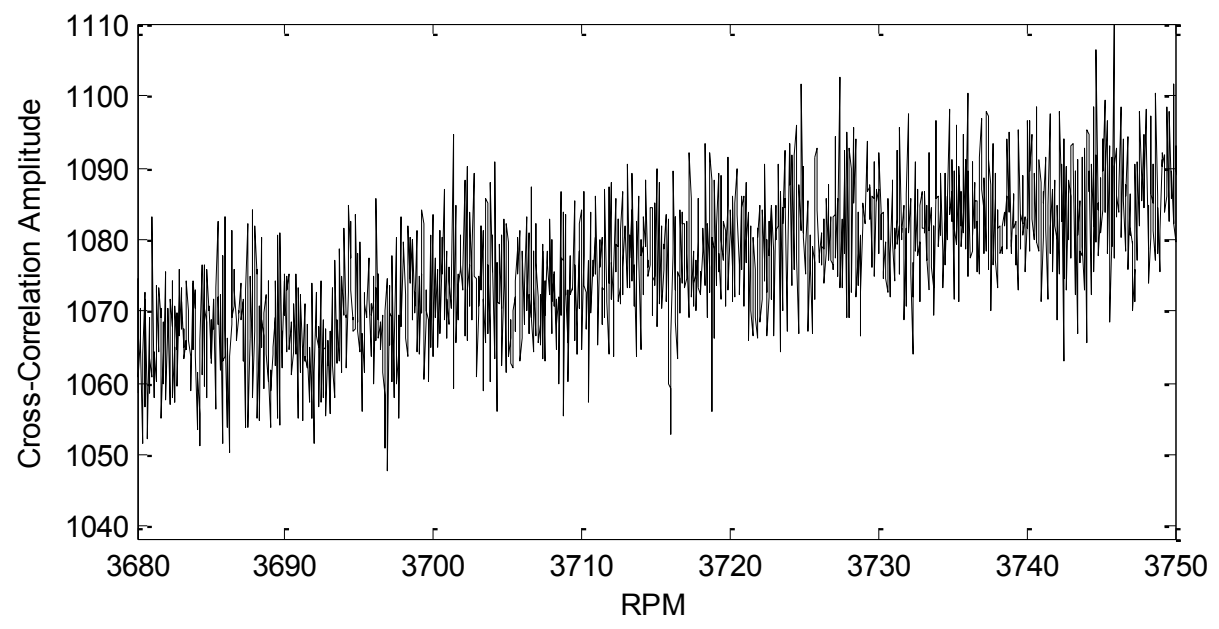

Figure 5.42: Cross Correlation, Blade 26, PS 80\% Span 20\% Chord, HL. 
In Figure 5.43 there is a large drop in covariance around 3705 RPM, which corresponds directly to the RPM band of maximum displacement in R2 for blade 26 at high loading, shown in Figure 5.44. Although the drop in covariance amplitude is small in comparison to the rev-to-rev variance in covariance amplitude, it is apparent that there is approximately a 30\% drop in average covariance amplitude from 3700-3705 RPM, and then a corresponding 30\% increase at 3705-3710 RPM.

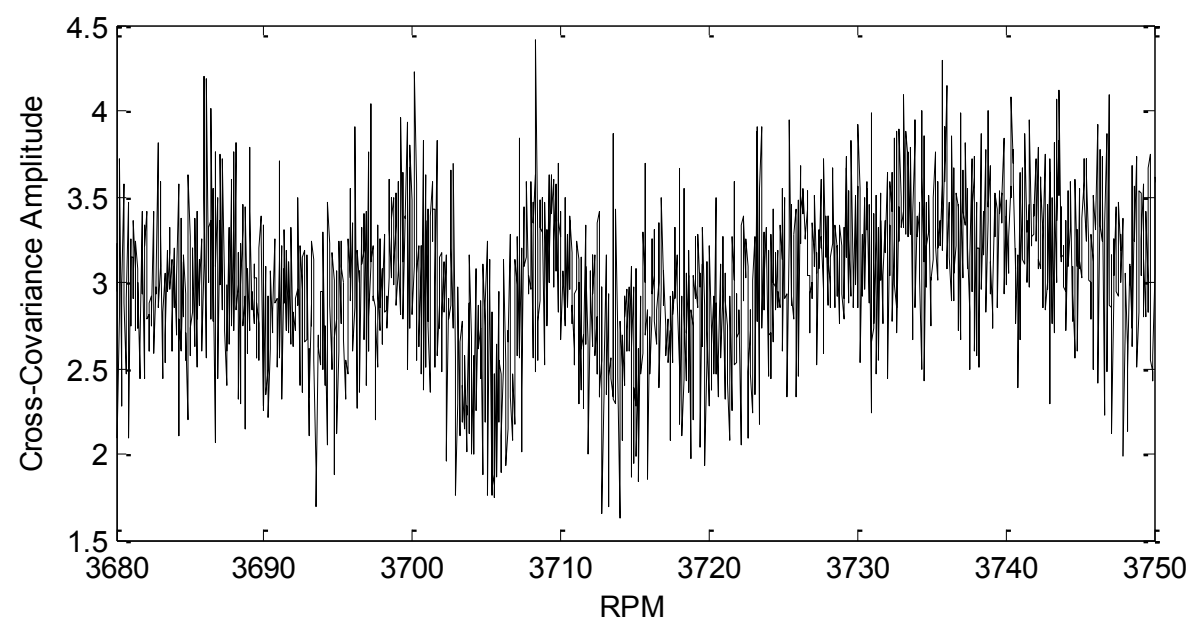

Figure 5.43: Cross Covariance, Blade 26, PS 80\% Span 20\% Chord, HL.

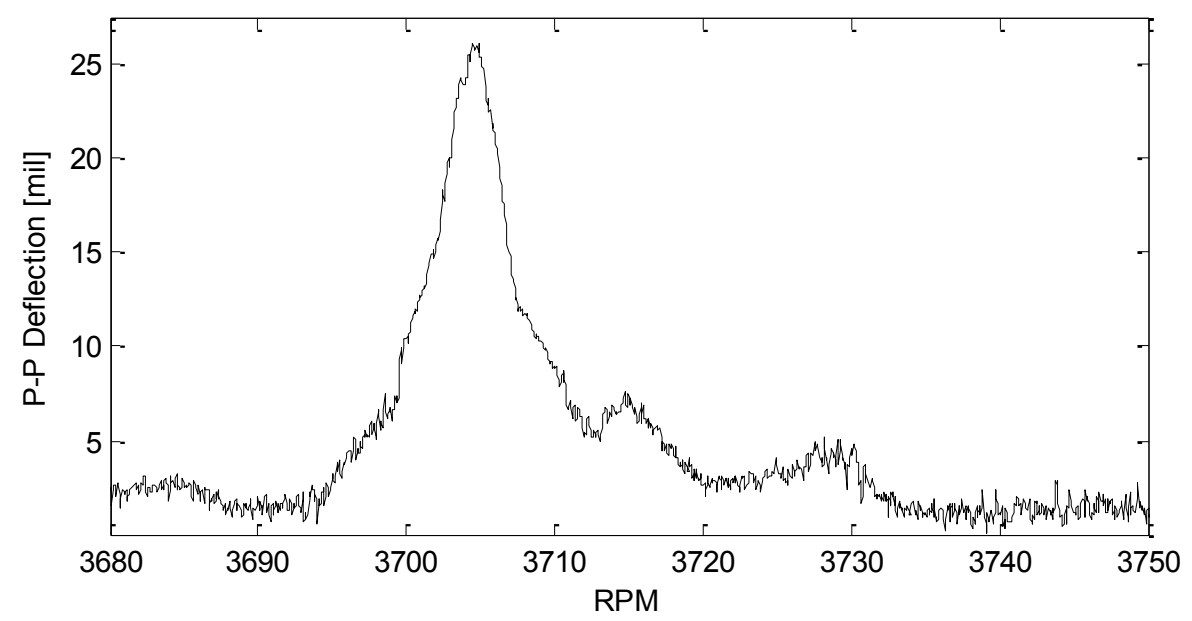

Figure 5.44: NSMS Peak to Peak Amplitude, Blade 26, HL. 
Figure 5.45 shows the pressure traces for blade 26 at high loading as measured by the Kulite on pressure side, $80 \%$ span, $20 \%$ chord. These compressor speeds were chosen at intervals to help illustrate how the blade passage shape is changing throughout the sweep. The red trace at $3671 \mathrm{RPM}$ is the pressure trace from the $1^{\text {st }}$ full revolution of data to which all successive pressure traces are compared for this analysis. The blue and cyan lines, at 3705 and 3710 RPM, respectively, show pressure traces of the blade in and around resonance, and the 3735 RPM black line shows the pressure trace near the end of the sweep, illustrating primarily how much the blade passage shape will normally change as TPR increases.

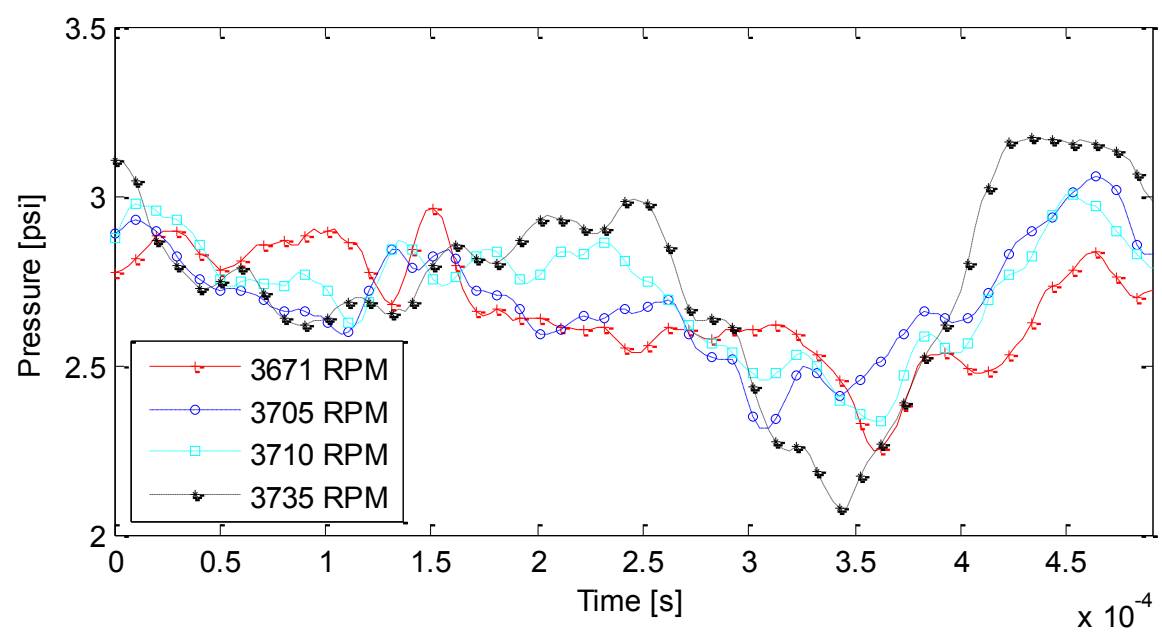

Figure 5.45: Rotor blade passage traces, Blade 26, PS 80\% Span 20\% Chord, HL.

To help determine whether or not this abrupt change in slope of cross correlation is random or attributed to $\mathrm{R} 2$ vibration, the same blade will also be analyzed through other sensors. Figure 5.46 shows the same cross covariance plot, but this time for a sensor at $50 \%$ span. The sensor for $50 \%$ span, $20 \%$ chord, which would have been directly under the sensor used to display previous data, was not functioning. In this figure, it is apparent that 
the same similar trough is apparent between approximately 3700-3710 RPM, before rising and then falling back to a normal level.

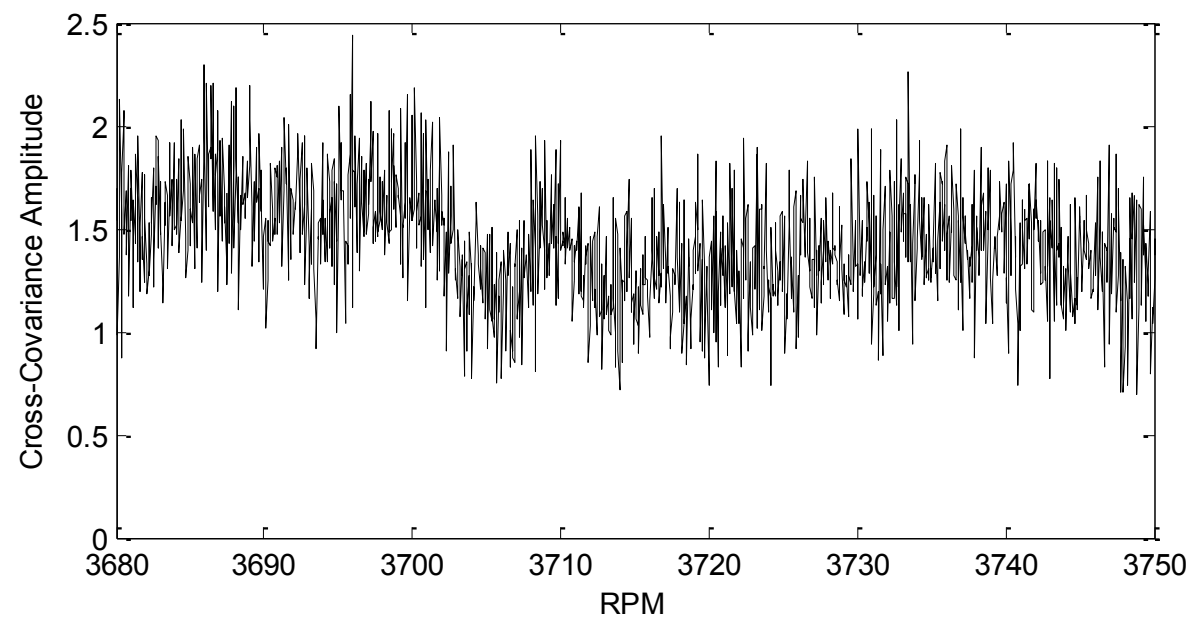

Figure 5.46: Cross Covariance, Blade 26, PS 50\% Span 10\% Chord, HL.

Staying at the same span as Figure 5.43, Figure 5.47 shows the cross covariance at a location downstream from the $20 \%$ chord, exhibiting the same drop in cross correlation from 3700-3710 RPM for the highest responding blade.

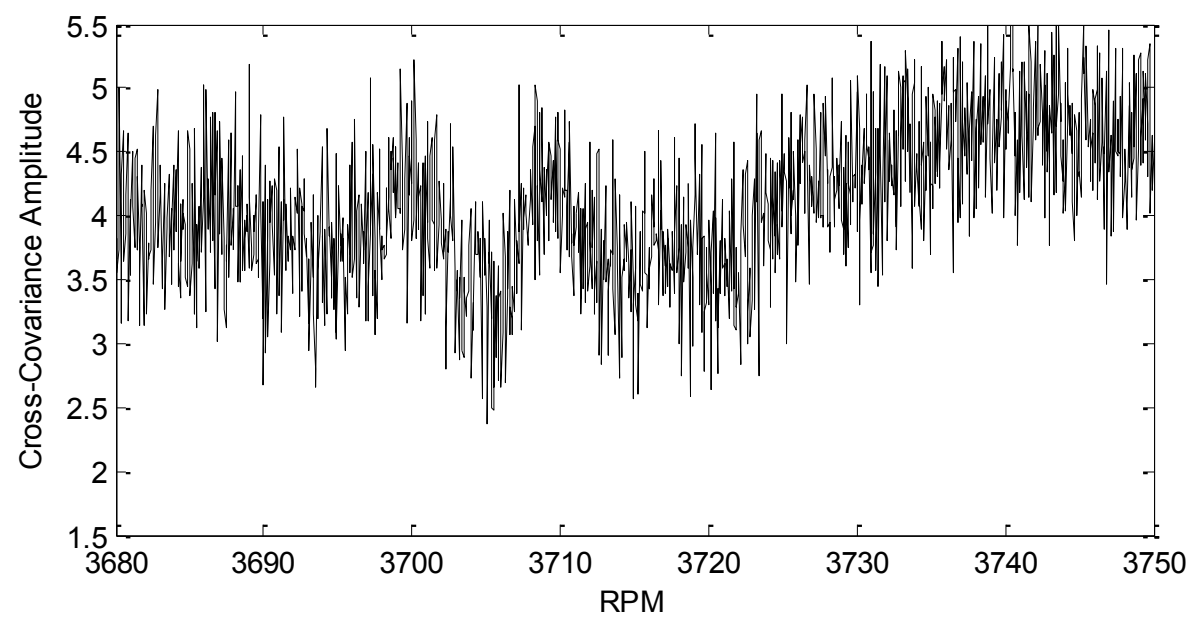

Figure 5.47: Cross Covariance, Blade 26, PS 80\% Span 40\% Chord, HL. 
The drop in cross covariance seems to disappear at nominal loading. In Figure 5.48, there is no drop in cross covariance around the position that would be expected from NSMS data shown in Figure 5.49. However, at this loading, there is approximately a $38 \%$ decrease in NSMS peak-to-peak response for this blade, which may be the largest driving factor determining whether or not the blade's response would be picked up in the cross covariance data.

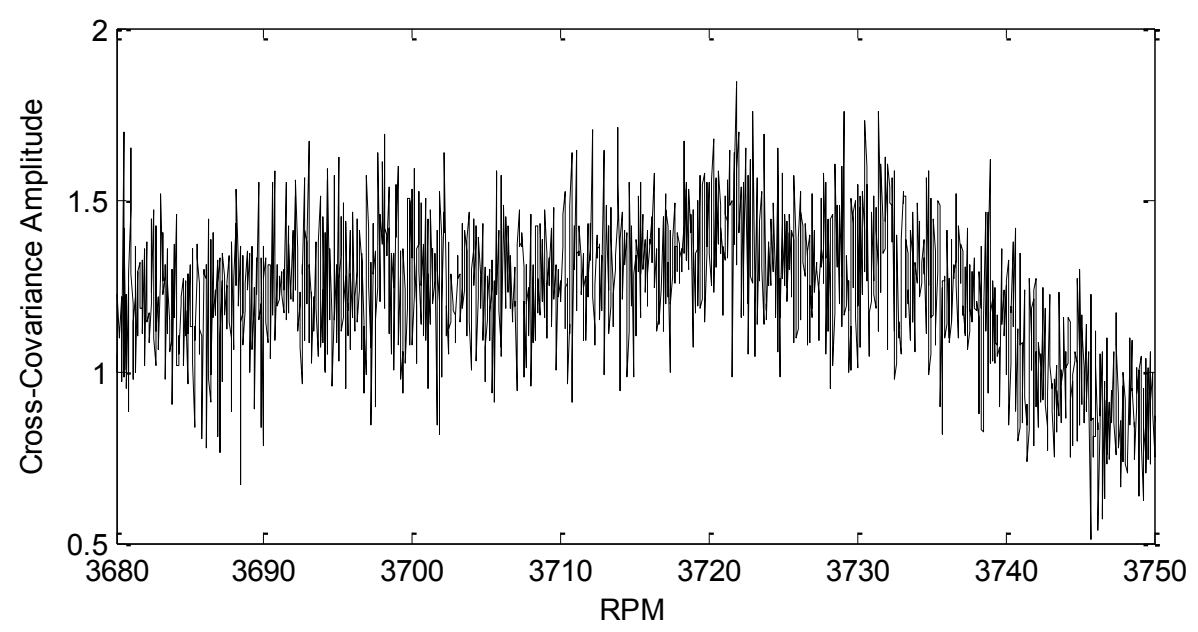

Figure 5.48: Cross Covariance, Blade 26, PS 80\% Span 20\% Chord, NL.

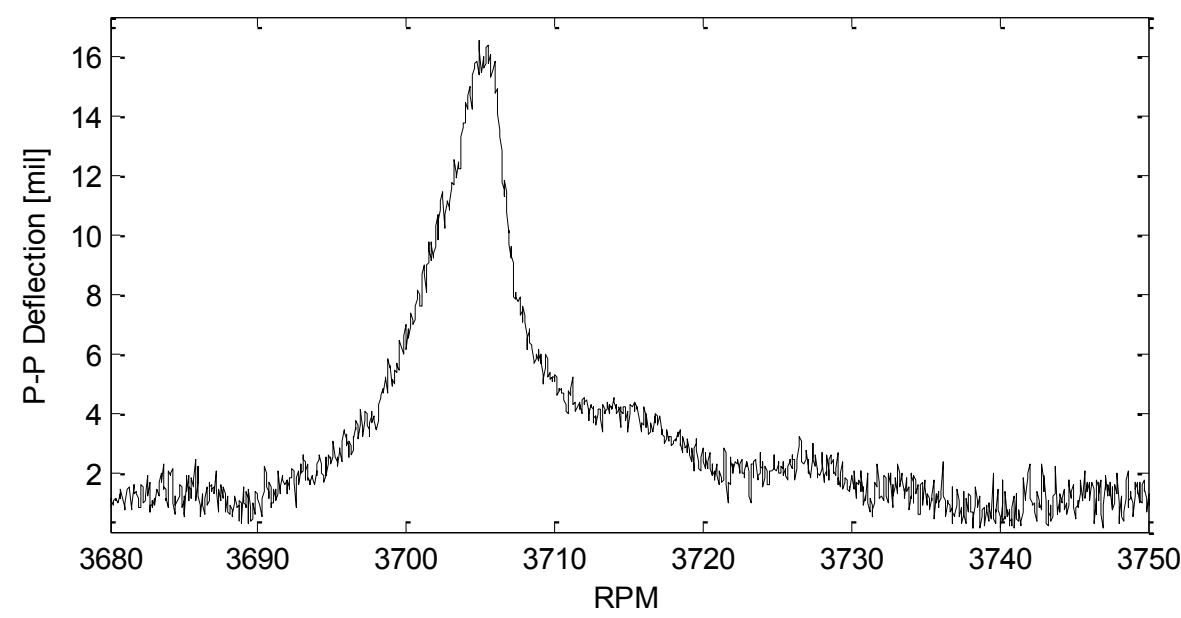

Figure 5.49: NSMS Peak to Peak Amplitude, Blade 26, NL. 
The difference in blade time of arrival, however, for blade 26 at NL between 3670 and 3733 RPM seems to be quite large, and there also seems to be a secondary harmonic strongly present in the waveform, as evidenced by the rise and fall in pressure from approximately .0001 and .0003 seconds, shown in Figure 5.50.

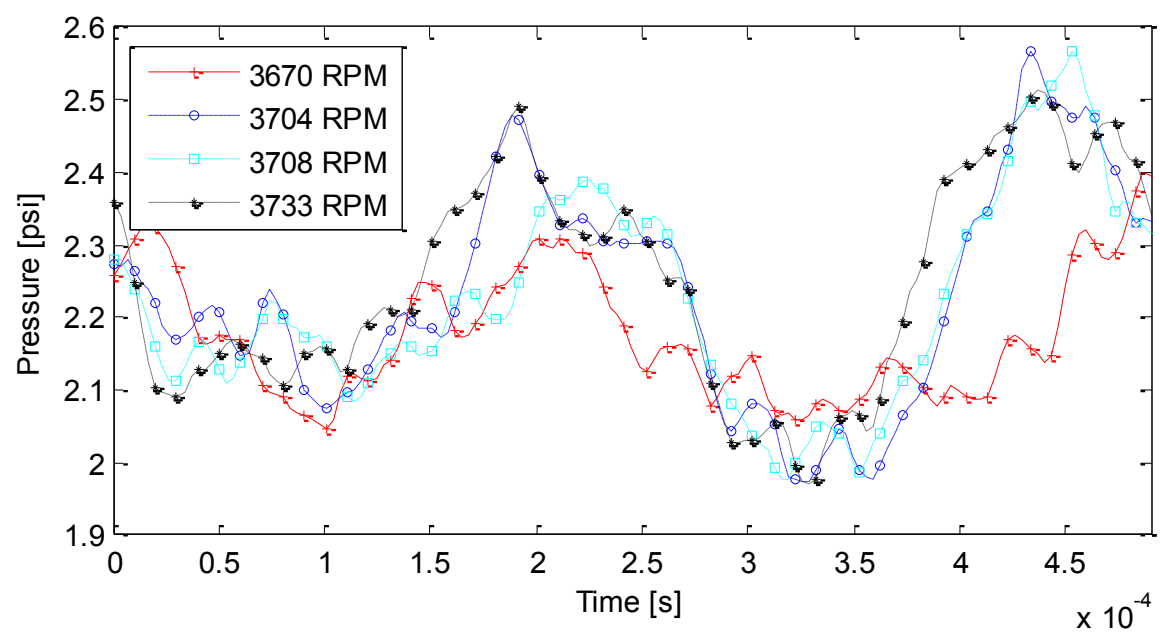

Figure 5.50: Rotor blade passage traces, Blade 26, PS 80\% Span 20\% Chord, NL.

The secondary harmonic that in the waveform data from the sensor located at $80 \%$ span $20 \%$ chord on the pressure side of the vane during NL is also strongly evident in the blade passage on the suction side of the stator vane during HL, evident in Figure 5.51. Correspondingly, there is no abrupt drop in the cross covariance term with this sensor, as well as all other suction side sensors, despite the large amplitude of the responding blade, shown in Figure 5.52. It is possible that this secondary harmonic could play a role in dampening out the components in the waveform that allow the covariance to drop during resonance. Also, it is not surprising that the suction side of the blade would measure a blade pressure trace waveform of a significantly different shape than on the pressure side of the 
vane, and it is perhaps the attenuation of the airflow within the vane passage that allows the secondary harmonic to strengthen.

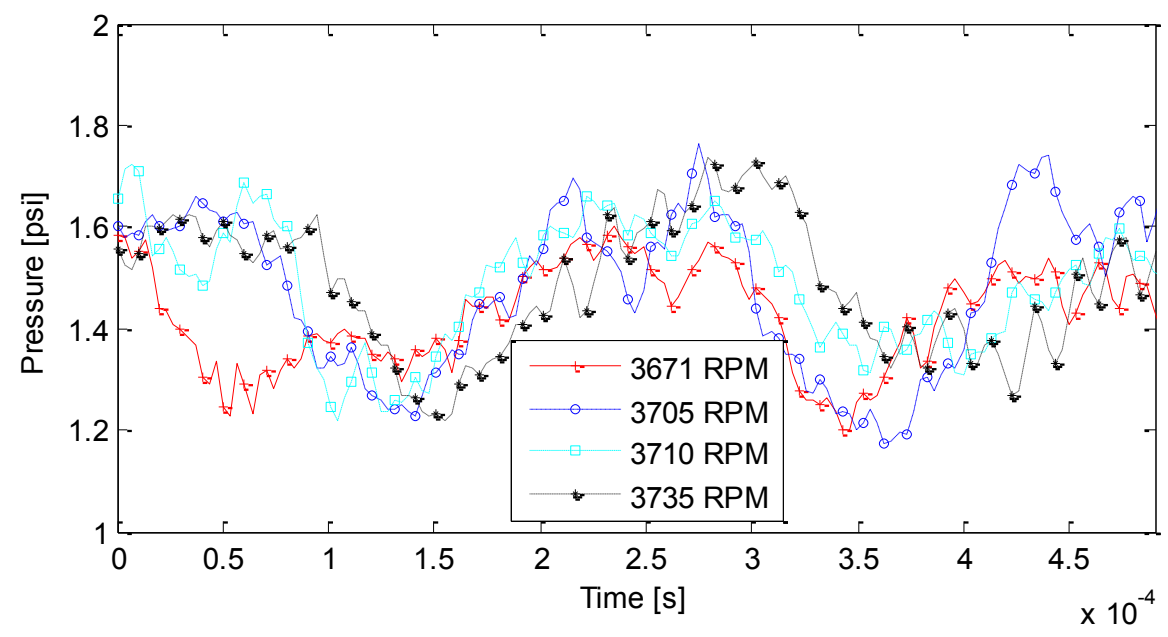

Figure 5.51: Rotor blade passage traces, Blade 26, SS 80\% Span 10\% Chord, HL.

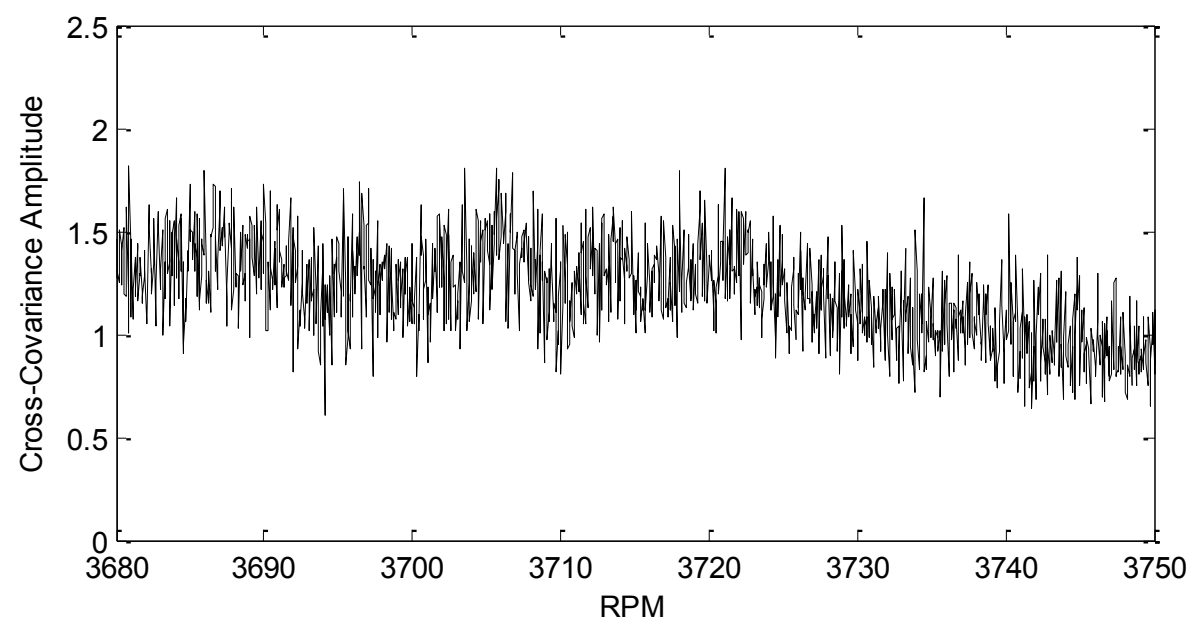

Figure 5.52: Cross Covariance, Blade 26, SS 80\% Span 10\% Chord, HL.

Turning now to the lowest responding blade, it is expected that the cross covariance term will not display any sharp changes in slope due to the fact that the lowest responding blade (13), even at high loading, has a peak-to-peak amplitude maximum of roughly $15 \%$ 
that of the highest responding blade (26). Figure 5.53 illustrates a slowly changing slope in the cross covariance of blade 13 as seen from a vane pressure side transducer, confirming these expectations. There is an inflection in the cross covariance around 3718 RPM, although this does not correspond to any change in the peak-to-peak amplitude of this blade as seen by NSMS in Figure 5.54. The blade passage waveform for blade 13 also seems to have a secondary harmonic present in the pressure rise around .0002 seconds in Figure 5.55, to go along with a negligible response in the cross covariance term.

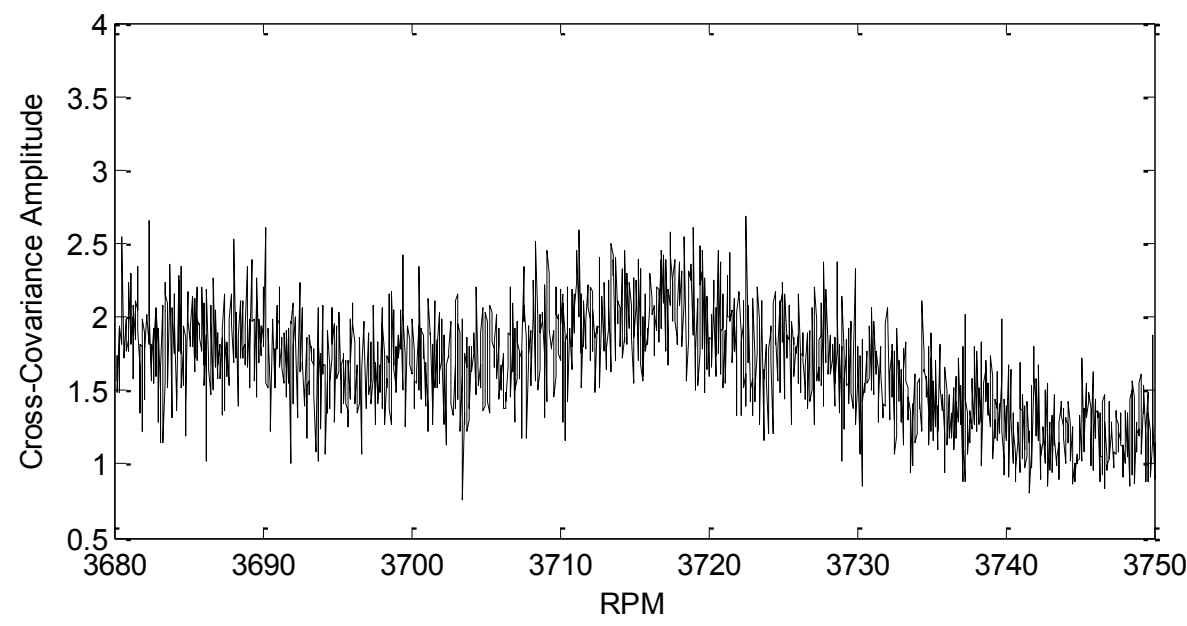

Figure 5.53: Cross Covariance, Blade 13, PS 80\% Span 20\% Chord, HL. 


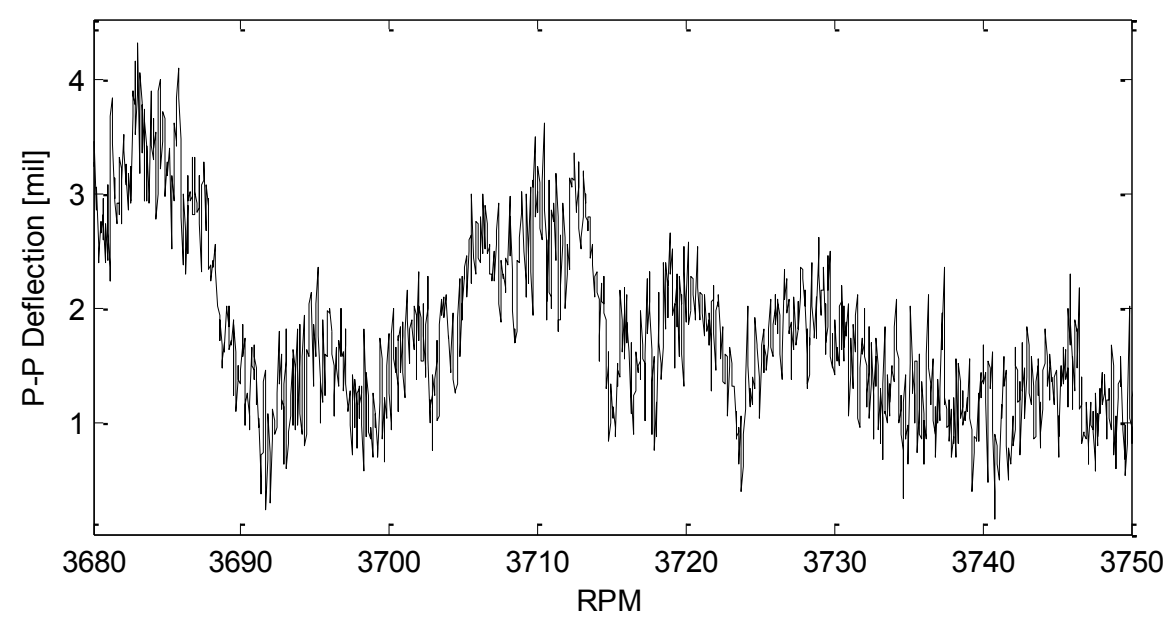

Figure 5.54: NSMS Peak to Peak Amplitude, Blade 13, HL.

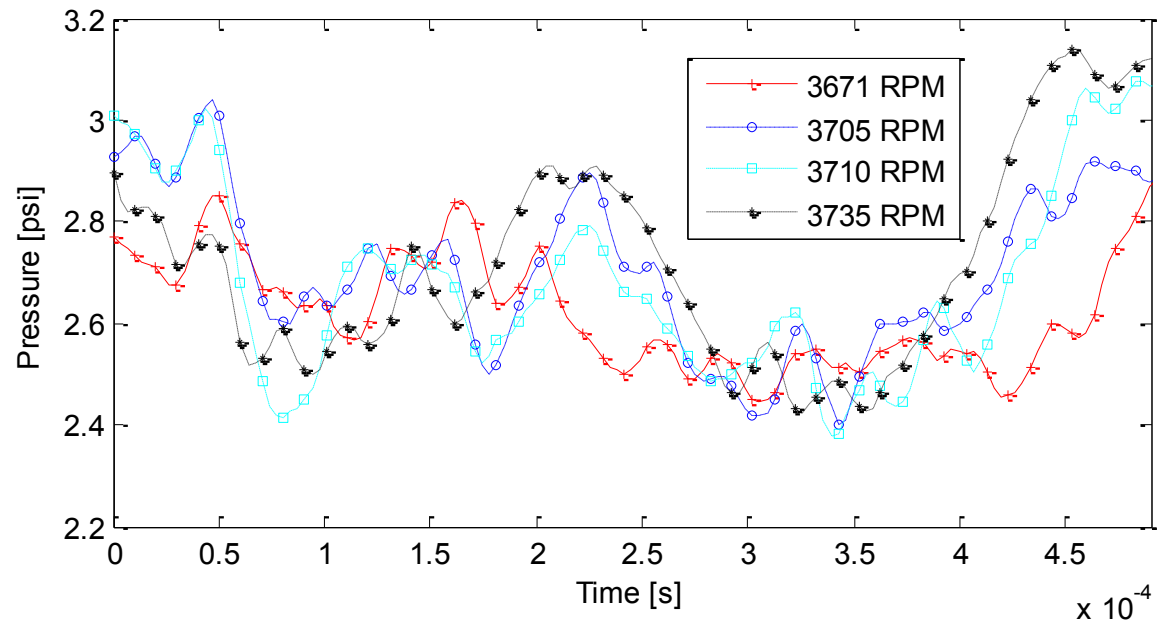

Figure 5.55: Rotor blade passage traces, Blade 13, PS 80\% Span 20\% Chord, HL.

Blade 8 at HL has a response similar in amplitude to that of blade 26 at NL, except that it is at a much higher RPM (higher frequency). Blade 8 also has a weak double-peak response, where it responds at two different frequencies due to blisk blade-to-blade and nodal diameter interactions. This causes it to respond slightly at around 3715 RPM and strongly at 3730 RPM in Figure 5.56. 


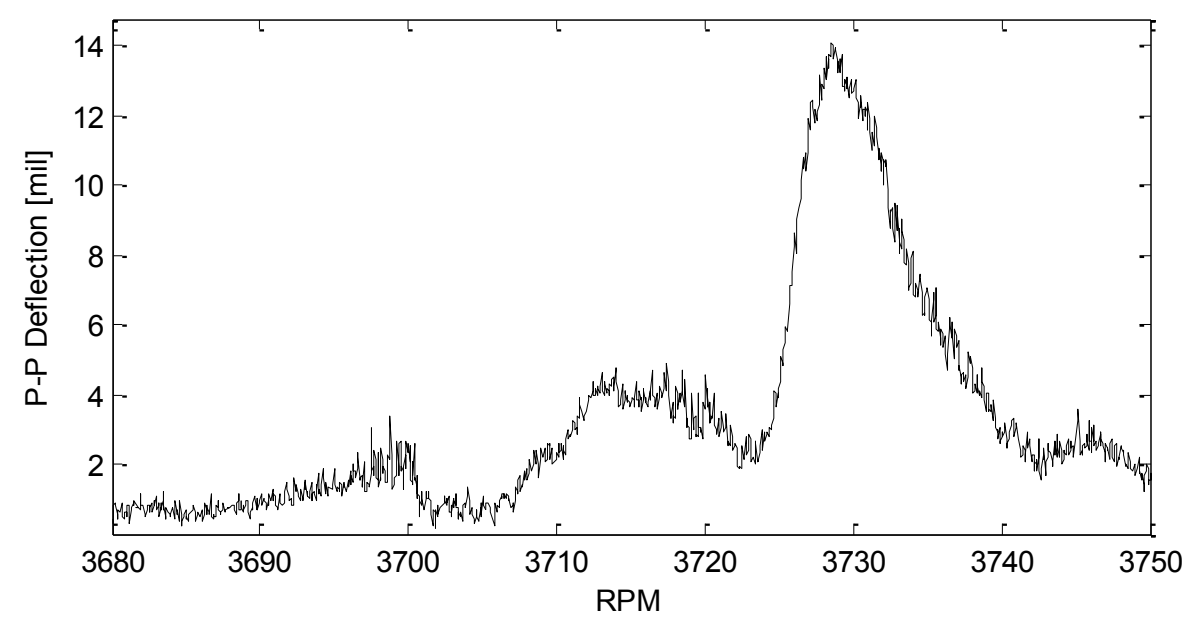

Figure 5.56: NSMS Peak to Peak Amplitude, Blade 8, HL.

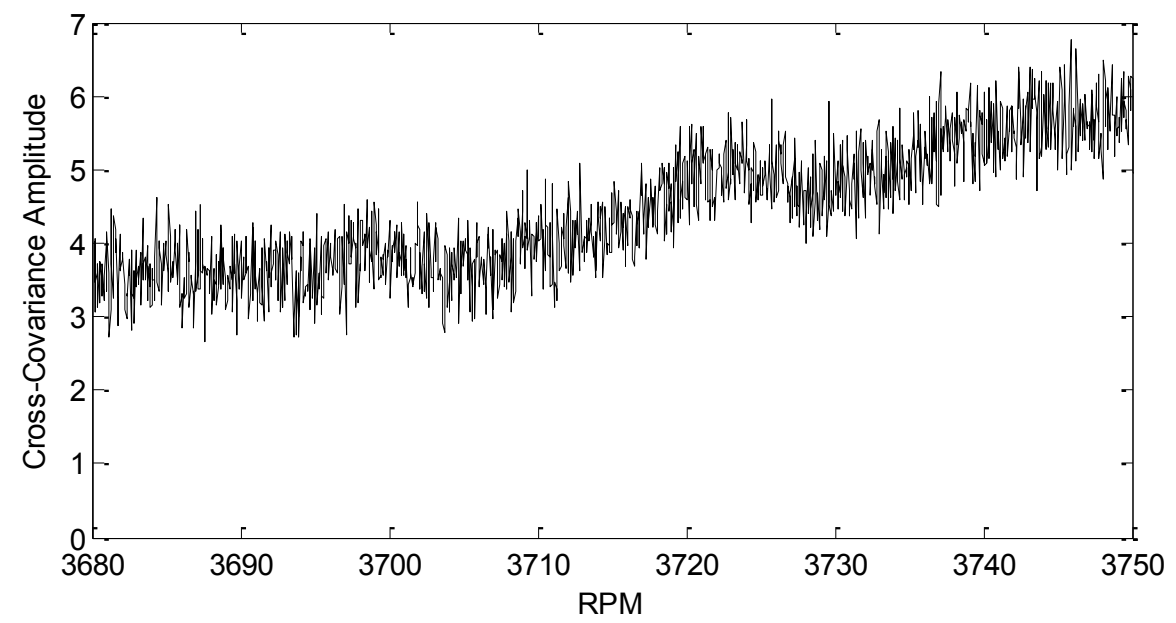

Figure 5.57: Cross Covariance, Blade 8, PS 80\% Span 20\% Chord, HL

The cross covariance the transducer measuring strongest response to blade vibration experiences a slight dip in the cross covariance term occurs around 3730 RPM in Figure 5.57. However, this dip is more gradual and less abrupt than the larger troughs seen in the pressure transducers recording the response of blade 26 at HL, although this smaller response would be expected if blade 8 is responding at less than half the peak to peak 
amplitude as blade 26. It may be too slight of a variation to draw significant conclusions from, as the dip in the covariance term at that speed may be merely coincidental.

Blade 22 is another high responder with what seems to be a similar drop in the cross covariance term, Figure 5.58, roughly equivalent to the width of the NSMS blade response profile, Figure 5.59. In this case, the covariance trough is very similar in shape to the one seen for blade 26 on the pressure side $80 \%$ span $20 \%$ chord transducer at high loading, and the trough width does not seem to be as wide as the blade response.

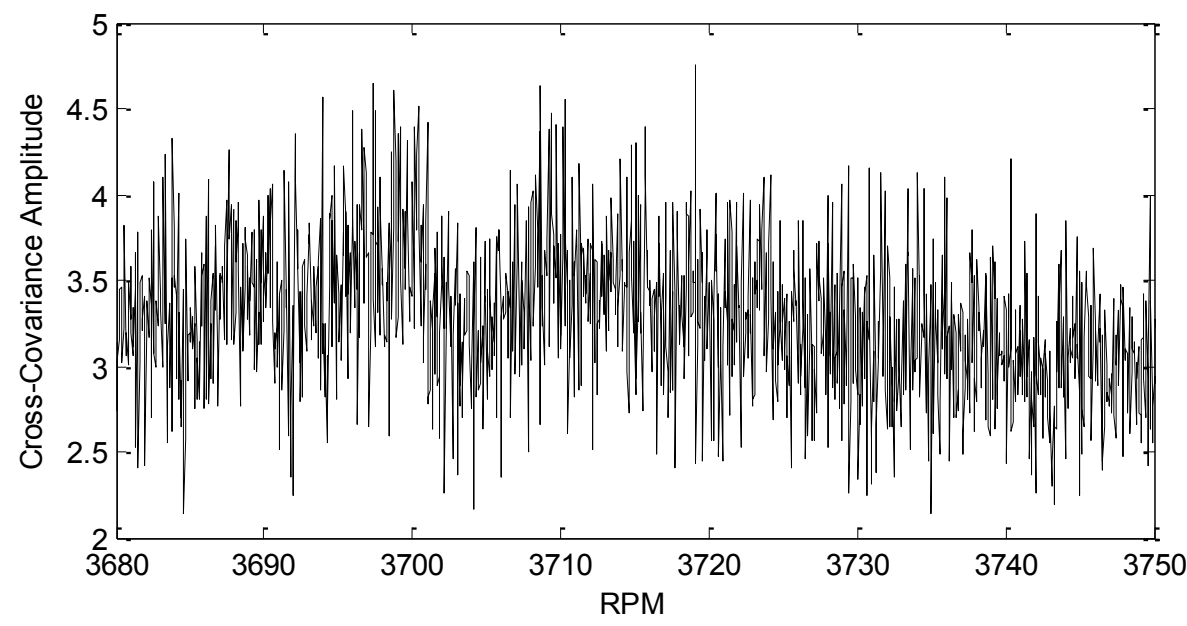

Figure 5.58: Cross Covariance, Blade 22, PS 80\% Span 20\% Chord, HL. 


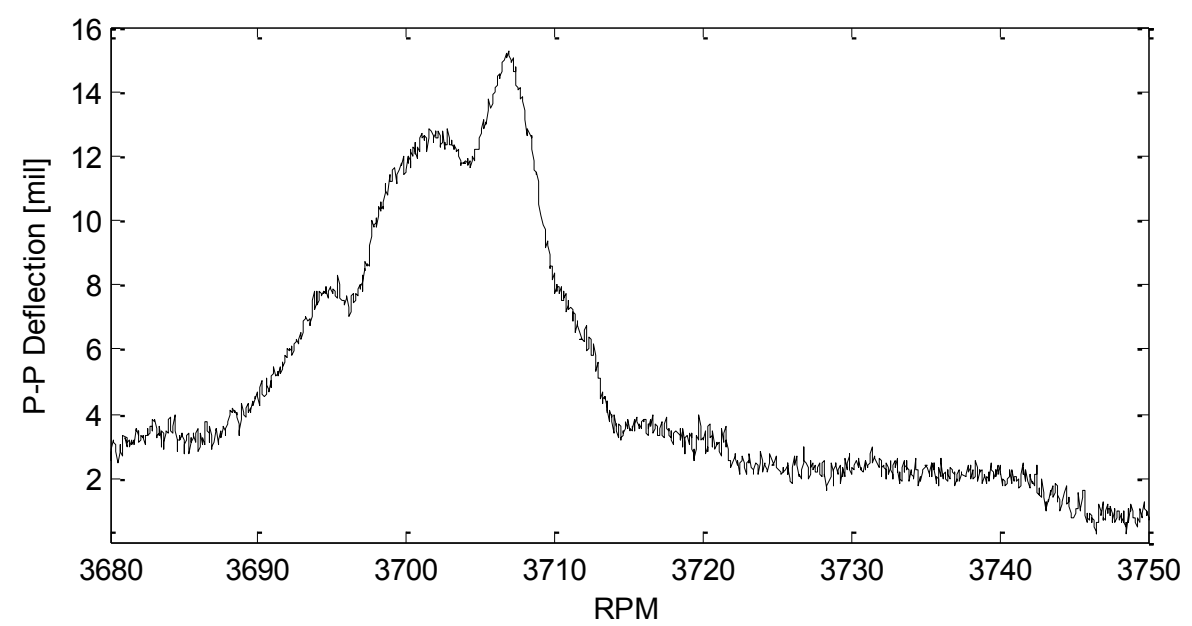

Figure 5.59: NSMS Peak to Peak Amplitude, Blade 22, HL.

Blade 27 is a blade that has an almost three-peak response, with two closely coupled responses at approximately 3697 and 3704 RPM, and a lower, more loosely coupled response at 3715 RPM in Figure 5.60. There is also a double trough in the cross covariance term at approximately the same RPM values seen in the NSMS response. However, the two troughs in Figure 5.61 seem to be more centered around 3705 and 3719 RPM, which is at a slightly higher speed than the third peak of the blade response and does not account for the first of the two closely coupled peaks. NSMS data displayed were no acquired in the same run as the stator Kulite data, which, due to some amount of non-repeatability in the NSMS data, could contribute to a slight shift in the RPM bands in which the blade responds. Also, it is worth noting that in blades that have a response less than about 10 mil, it is usually impossible to detect any noticeable change in the cross covariance term as a function of RPM that is at all attributed to blade vibratory motion. Because of this, it is more likely that the second trough in the covariance plot would be due to variation in the 
calculated covariance from other factors that coincidentally happens to be near the same RPM as the third peak in blade 27.

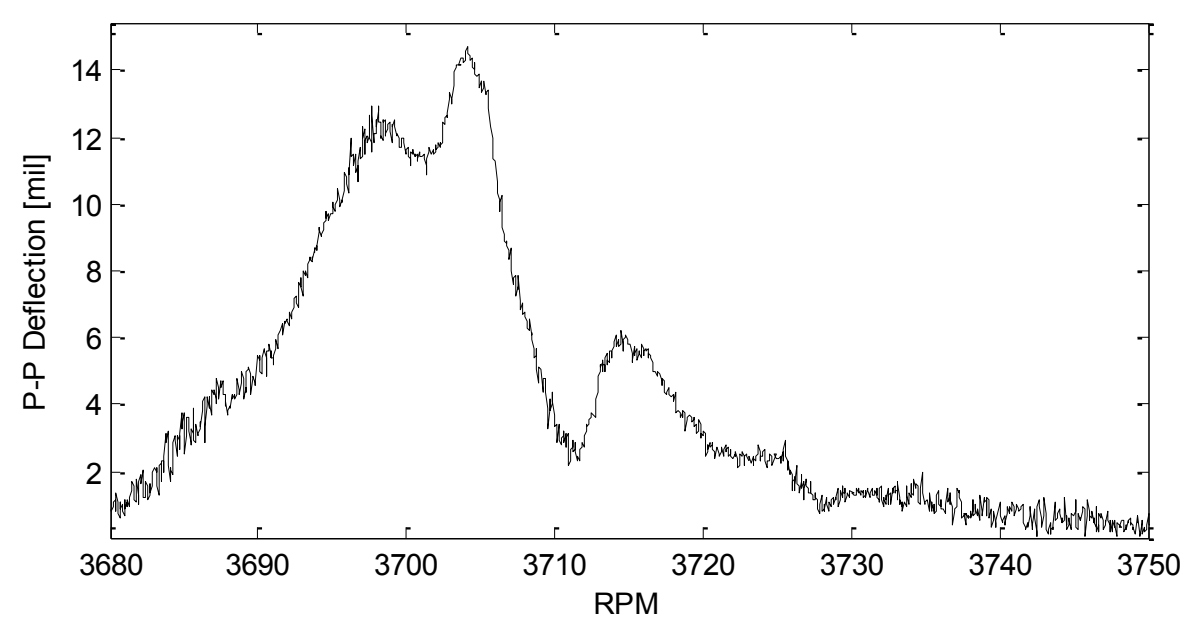

Figure 5.60: NSMS Peak to Peak Amplitude, Blade 27, HL.

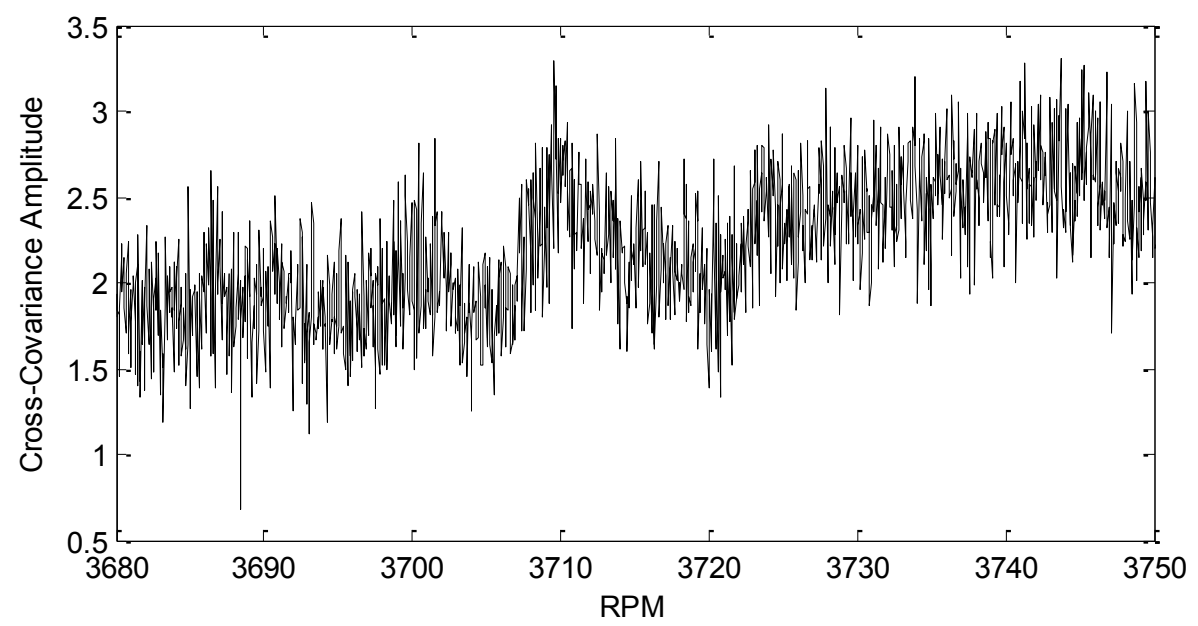

Figure 5.61: Cross Covariance, Blade 27, PS 80\% Span 20\% Chord, HL.

There is an inherent waviness, or fluctuation in the overall trend of the cross covariance (or, for that matter, standard deviation and cross correlation) that is apparent in all sensors regardless of RPM, which makes the measurements of cross covariance amplitude drops difficult to compute. 
The cross covariance analysis was conducted on casing wall Kulites, and the results were largely inconclusive. There appeared to be no direct evidence of blade vibration that was noticeably aligned with NSMS data, and it is therefore not shown. It is suspected that the lower frequency response of the Kulite sensors in the casing make this measurement difficult to detect. 


\section{CHAPTER 6. CONCLUSIONS}

The work presented in this thesis has focused on compressor aeromechanics in both a centrifugal and axial compressor. The centrifugal compressor project is at the early stages of development. A test cell layout has been created, and a LDV inlet flow experiment has been designed to help understand the effects of inlet distortion on a forced response mode in a centrifugal compressor. The axial compressor project allowed for the acquisition of aeromechanics data related to the forced response of an embedded stage in an axial compressor, from the aerodynamic forcing functions both upstream and downstream of the rotor as well as the mechanical response. It also allowed for the development of advanced data analysis techniques that allow pressure transducers to detect engine-order forced response modes. A large library of data has now been acquired that will shed insight into forced response as well as facilitate the calibration and fine-tuning of unsteady aeromechanics computer simulations.

\subsection{Setup for Centrifugal Compressor Experiments}

The facility for the centrifugal aeromechanics experiments has been outfitted with a brand new control station, custom built from wood and 80/20 aluminum strut, with ample room for expansion. It currently houses all of the electronics that have been purchased for compressor instrumentation. 
Major equipment includes Scanivalve electronics that have been purchased for steady pressure measurement and Agilent multiplexers temperature measurement. A NI-PXI chassis was purchased for health monitoring purposes as well as analog output for valve control, and a Precision filter amplification system has been purchased for unsteady strain gauge and Kulite measurements.

A supercritical air ejector that meets all airflow requirements for the APU inlet flow experiment is compatible with the high pressure air infrastructure already present at Zucrow laboratories and has been installed. It is remotely controlled by a TESCOM regulator with a $\mathrm{C}_{\mathrm{v}}$ of 12 that itself is remotely controlled by an ER-3000 electronic air regulator through LabView. The ejector can be throttled and maintains a steady suction airflow at design conditions of $5.5 \mathrm{lbm} / \mathrm{s}$ to within $+/-1 \%$.

The APU-style Honeywell inlet was modified to provide generous optical access for LDV measurements and is easily taken apart for cleaning. The windows are scratch proof and the structure so far has been able to handle the pressure loads and vibrations of flow testing with no structural issues, as expected from ANSYS predictions. It is expected to meet all structural expectations once compressor testing commences.

An air and oil system has been designed to accommodate the Honeywell SSCC compressor and Cotta gearbox that will transmit power to the compressor. The oil system has the capability to heat 30 gallons of oil to $240^{\circ} \mathrm{F}$ in less than 20 minutes, and it supplies the oil at three different pressures and flow rates to the compressor. It includes an external unistrut enclosure housing heat exchangers to dump waste heat from the compressor and gearbox. The oil system will be instrumented with pressure 
transducers and thermocouples to ensure proper flow rates and temperatures for steady compressor operation.

The secondary air system will consist of 4 ER-5000 electronic air regulators that will supply 3 different air pressures at 3 different flow rates as well as 1 suction pressure to the rig. They will be throttled through the range of required pressures and flow rates that the rig will experience during operation. Two exhaust flow rates will be measured with venturis, and three of the outgoing air flows will be throttled through the use of high-temperature globe valves. The entire system will be automated and monitored through LabView and is currently under construction at the time this thesis is written. The final design of the air and oil system may change upon final delivery of the SSCC compressor rig.

\subsection{Aeromechanics Axial Compressor Research}

Several different measurement techniques were employed on the Purdue 3-stage axial compressor around Rotor 2 to fully measure the flow field characteristics pertaining to a Campbell diagram crossing of the 44EO excitation of R2 1T vibratory mode. These data are needed for predictive tool validation and calibration.

\subsubsection{Acquisition and Characterization of Forcing Functions}

A steady compressor performance map was taken at speed lines around resonance, and 50-point vane traverses to acquire $\mathrm{P}_{\mathrm{o}}$ and $\mathrm{T}_{\mathrm{o}}$ profiles were conducted at the corrected speed corresponding to rotor $21 \mathrm{~T}$ vibration both upstream and downstream of Rotor 2. Stator wake and rotor 2 static pressure forcing functions were 
measured and quantified. Two loading lines were taken at NL and HL that span the mechanical speeds in all data. Casing temperature and detailed inlet pressure measurements were acquired, giving a detailed suite of information that can be used in CFD simulations, specifically aeromechanics simulations of interest to the GUIde IV consortium. Wake widths and depths increase significantly at HL compared to NL, and the $1^{\text {st }}$ harmonic frequency components of an FT of the wake shape increase significantly as well, and this is the driver in increasing rotor 2 response.

NSMS tip-timing data were acquired over rotor 2 at two different loadings and through acceleration and deceleration to measure R2 blade tip deflection. Damping coefficients, peak-to-peak amplitudes, phase information, and frequency response of the blades as well as the ND characteristics of the R2 blisk were measured. Blade vibration amplitudes more than double at HL compared to NL, but the frequencies at which the blades vibrate tends to remain the same. The aerodynamic damping nearly doubles as well. This can be directly attributed to the increase in magnitudes of the vortical and potential forcing functions described previously.

A 20-point cross-film hotwire traverse was conducted at 50\% and $80 \%$ span, for NL and HL, downstream of R2, and a 40 -point traverse, $50 \%$ and $80 \%$ span, for NL and HL, downstream of S1, were acquired. Each point consisted of at least 500 revolutions of data acquired at $74 \% \mathrm{~N}_{\mathrm{c}}$. Sweeps were also conducted for both loadings, spans, and locations through R2 1T. S1 wakes and S2 potential fields were measured and are useful for the implementation of CFD aeromechanics studies, and this data agreed with wake data obtained by rake measurements. 
Unsteady lift was measured through the use of Kulites installed on the suction and pressure side surfaces of a downstream stator, and showed large changes in lift shapes as loading changed. Additionally, through Fourier Transform analysis, the stator pressure fields are shown to be primarily influenced by the upstream rotor 2 but also some contribution from downstream rotor 3 exists in the form of several harmonic orders of blade pass frequency. Additionally, many combinations of blade number harmonics are detected, suggesting strong reflections as well as constructive/destructive interference from rotor-rotor interactions

Similar results were found with over rotor casing mounted Kulites, where FT spectra from all 3 rotors were detected, and varied in strength depending on axial location. The harmonics related to $\mathrm{R} 1$ and $\mathrm{R} 3$ were dependent on circumferential position, suggesting that stator potential field plays a strong role in amplifying or damping the propagation of higher order pressure harmonics throughout the compressor. Blade pass pressure signatures were analyzed for each sensor as well, showing that the R2 blade passage event was strongest at the leading edge and center chord Kulite positions, but become smoothed out and more heavily influenced by stator 2 interactions at downstream locations.

\subsubsection{Measurement of R2 Blade Vibration through Pressure Transducers}

LINSUB was used to estimate whether the Kulite pressure transducers would be able to detect acoustic signatures from R2 1T, and it showed that the acoustic pressure fluctuations would be on the order of the uncertainty in the Kulite pressure measurement. The amplitude of the pressure wave will increase with compressor 
rotational speed so while the pressure signature associated with blade vibration may be small in this research vehicle, it will be significant at actual engine operating conditions.

Fast response instrumentation have been used to characterize the unsteady flow field upstream, downstream, and above rotor 2. Casing Kulites and vane-mounted Kulites on S2 measured unsteady pressures at steady operating points just outside of R2 resonance, and throughout several different sweeps through resonance, both accelerating and decelerating. Data processing in both the frequency and time domain show that signatures of the acoustic signature generated by R $21 \mathrm{~T}$ vibration have been recorded through the use of Stator 2 mounted pressure transducers. Waterfall Fourier analysis showed no trace of R2 1T, in case-mounted Kulites, however, and it was concluded that velocity fluctuations from R2 $1 \mathrm{~T}$ are too small in comparison to blade pass signatures to stand out in a Fourier analysis for these sensors. Through the calculation of Doppler shifted vibration frequencies, spectra related to R2 $1 \mathrm{~T}$ have been detected in the $2^{\text {nd }}$ and $3^{\text {rd }}$ harmonics of BPF. Additionally, through the use of cross covariance blade-to-blade similitude analysis, individual blade vibration has been detected. It is to the author's knowledge that this is the first time in the open literature that such measurements of an Engine-Order forced response mode has been detected through the particular data processing methods employed in this study. It is possible that the detection of Engine Order forced response at part speed compressor operation through the use of pressure transducers could have an impact on the way blade vibrations are measured in turbomachinery, as this measurement method is significantly simpler than other advanced vibration measurements, such as rotating instrumentation/laser-based measurements. 


\subsubsection{Additional Aeromechanics Data}

Stator vibrometry studies were conducted at WPAFB, and were useful in determining safe operating speeds for the compressor and are useful to validate ANSYS mode analysis previously conducted. Additionally, laser scans, illustrations of which are included in the appendix, were conducted on all three rotors, and the data can be used for future mistuning analysis.

\subsection{Suggestions for Future Work}

The amount of unsteady data gathered in all of this research is tremendous, and, including the data sets that were not used for final analysis in this thesis (different sampling rates, clocking configurations, etc.) amounts to well over 50 GB in binary data. Hotwire data could be used for rotor wake variability studies, as well as sent through the full suite of similitude analysis (cross correlation/covariance, etc.). It would be interesting to tie together stator 2 unsteady loading to rotor blade variability, as well as correlate it to the different response amplitudes and characteristic shapes of Rotor 2 blades. The laser scan data has not been used yet by this lab for a mistuning analysis, but that is something that could be looked into as well.

Probably only $10 \%$ of the Agilis NSMS software has been used to analyze the data coming from Rotor 2 taken for this thesis. There also exists R3 and R1 data taken by a previous student on the same project that can be analyzed for many different things, in particular the damping modes of the blisk assembly. Damping information, combined with unsteady aerodynamics, could possibly predict the actual changes in amplitude response as a function of compressor loading. 
The cross covariance analysis could be improved further. Currently, the blade parsing, especially at the stator, is not carried out very precisely. It would be nice to tie in the hotwire rotor exit wake data to give a much better estimate of the time of arrival of blade wake interaction with S2 as a function of chord position. Also, currently, the analysis methods only consider the highest possible covariance value which is a function of lag offset. This would work against the notion that blade pressure wake time of arrival is a large component of the drops in covariance. However, currently, it doesn't always seem that the lag offset corresponding to the greatest covariance value necessarily means that the blade pass pressure vectors are perfectly aligned — this is most likely due to the incredibly "muddied" pressure fluctuation, especially at the stator suction side wall. Additionally, the fact that the covariance term used to analyze the similitude of blade passing events is only a function of the piecewise product of both blade passage vectors may mask details in the minutiae of the blade passage pressure traces that might reflect the influence of the forced response acoustic signatures. Therefore, possibly a weighted function of the blade pass, one that could still be based on the product of the two vectors, but weights the differences in blade pressure TOA in the form of a large coefficient, might make it easier to notice changes in the blade passage shape. 
LIST OF REFERENCES 


\section{LIST OF REFERENCES}

Ariga, I., Masuda, S., Ookita, A., 1987. "Inducer Stall in a Centrifugal Compressor With Inlet Distortion." ASME Journal of Turbomachinery, 109, 27-35.

Baumgartner, M., Kameier, F., Hourmouzaiadis, J., 1995. "Non-Engine Order Blade Vibration in a High Pressure Compressor," Presented at the ISABE International Symposium on Airbreathing Engines, Melbourne, Australia, September 10-15.

Belz, J., May, M., Siemann, J., Seume, J. R., Voigt, C., Böhmer, H., Grüber, B., 2013. "Excited Blade Vibration For Aeroelastic Investigations of a Rotating Blisk Using PeizoElectric Macro Fiber Composites," Proceedings of ASME Turbo Expo 2013, San Antonio, TX., June 3-7.

Brossman, J.R., 2012. An Investigation of Rotor Tip Leakage Flows in the Rear Block of a Multistage Compressor, PhD Dissertation, Purdue University, West Lafayette, IN.

Bruun, H. H., Khan, M. A., Al-Kayiem, H. H., Fardad, A. A., 1990. "Velocity Component Measurements by X Hot-Wire Anemometry,” Meas. Sci. Technol., 1, 1314-1321. 
Buffum, D. H., 1995. "Blade Row Interaction Effects on Flutter and Forced Response," AIAA Journal of Propulsion and Power, 11-2, 205-212.

Cousins, W. T., Dalton, K. K., Andersen, T. T., Bobula, G. A., 1994. "Pressure and Temperature Distortion Testing of a Two-Stage Centrifugal Compressor." ASME Journal of Engineering for Gas Turbines and Power, 116, 567-573.

Cumpsty, N. A. Compressor Aerodynamics. Malabar, FL: Krieger Pub., 2004. Print.

Engeda, A., Kim, Y., Aungier, R., Direnzi, G., 2003. "The Inlet Flow Structure of a Centrifugal Compressor Stage and Its Influence on the Compressor Performance," ASME Journal of Fluids Engineering, 125, 779-785.

Ewins, D. J., 1969. “The Effects of Detuning Upon the Forced Vibrations of Bladed Disks,” Journal of Sound and Vibration, 9, 65-79.

Freund, O., Bartelt, M., Mittelbach, M., Montgomery. M., Vogt, D. M., Seume, J. R., 2013. "Impact of the Flow on an Acoustic Excitation System for Aeroelastic Studies," ASME Journal of Turbomachinery, 135, 031033-1 - 031033-9.

Fridh, J., Laumert, B., Fransson, T., 2013. "Forced Response in Axial Turbines Under the Influence of Parial Admission," ASME Journal of Turbomachinery, 135, 041014-1 041014-9. 
Gancedo, M., Gutmark, E., Mohamed, A., Guillou, E., (2012). "PIV Measurements of Flow in Recirculation Cavities at the Inlet of a Centrifugal Compressor," 50th AIAA Aerospace Sciences Meeting, Nashville, TN, January 9-12.

Gill, J. D., Capece, V. R., 2004. "Experimental Investigation of Fllutter in a Single Stage Unshrouded Axial-Flow Fan," Presented at $42^{\text {nd }}$ AIAA Aerospace Sciences Meeting and Exhibit, Reno, NV, January 5-8.

Hall, K. C., Silkowski, P. D., 1997. “The Influence of Neighboring Blade Rows on the Unsteady Aerodynamic Response of Cascades," ASME Journal of Turbomachinery, 119, 85-93.

Hanson, D.B., 1993. "Mode Trapping in Coupled 2D Cascades-Acoustic and Aerodynamic Results," Presented at 15 th AIAA Aeroacoustics Conference, Long Beach, CA, October 25-27.

Im, H., Zha, G., 2013. "Flutter Prediction of a Transonic Fan with Travelling Wave Using Fully Coupled Fluid/Structure Interaction," Proceedings of ASME Turbo Expo 2013, San Antonio, TX, June 3-7.

Kammerer, A., Abhari, R. S., 2010. "Blade Forcing Function and Aerodynamic Work Measurements in a High Speed Centrifugal Compressor With Inlet Distortion," ASME Journal of Engineering for Gas Turbines and Power, 132, 092504-1 - 092504-11. 
Kaneko, Y., 2013. "Study on Transient Vibration of Mistuned Bladed Disk Passing Through Resonance," Proceedings of the ASME Turbo Expo 2013, San Antonio, TX, June $3-7$.

Kang, K., Shin, Y., Kim, K., and Lee, Y., 2010. "Inlet Distortion of a Centrifugal Compressor with a Circular-Sectioned 90-Degree Bend and Its Influence on the Performance." Proceedings of the ASME 2010 3rd Joint US-European Fluids Engineering Summer Meeting and 8th International Conference of Nanochannels, Microchannels, and Minichannels, Montreal, Canada, August 1-5.

Kaza, K. V., Kielb, R. E., 1981. "Flutter and Response of a Mistuned Cascade in Incompressible Flow," AIAA Journal, 20-8, 1120-1127.

Key, N.L., "Secondary Flows," ME 433 Class Notes, Lecture 28, Purdue University, November 2012.

Kim, Y., Engeda, A., Aungier, R., Direnzi, G., 2001. "The Influence of Inlet Flow Distortion on the Performance of a Centrifugal Compressor and the Development of an Improved Inlet Using Numerical Simulations," Proceedings of the Institution of Mechanical Engineers, Part A: Journal of Power and Energy, 215, 323-338. 
Kim, Y., Koch, J., 2004. "Design and Numerical Investigation of Advanced Radial Inlet for a Centrifugal Compressor Stage." Proceedings of ASME 2004 International Mechanical Engineering Congress and Exposition, Anaheim, CA, November 13-19.

Kurkov, A. P., 1981. "Flutter Spectral Measurements Using Stationary Pressure Transducers," ASME Journal of Engineering and Power, 103, 461-467.

Lawless, P.B., Fleeter, S., 1994. "Effect of Controlled Inlet Distortions on Rotating Stall Inception in a Low Speed Centrifugal Compressor." 30th AIAA/ASME/SAE/ASEE Joint Propulsion Conference, Indianapolis, IN, June 27-29.

Leichtfuß, S., Holzinger, F., Brandstetter, C., Wartzek, F., Schiffer, H. P., 2013. “Aeroelastic Investigation of a Transonic Research Compressor," Proceedings of ASME Turbo Expo 2013, San Antonio, TX, June 3-7.

Li, H. D., He, L., 2005.’Blade Aerodynamic Damping Variation with Rotor-Stator Gap: A Computational Study Using Single-Passage Approach,” ASME Journal of Turbomachinery, $127,573-579$.

Mengle, V., 1990. “Acoustic Spectra and Detection of Vibrating Rotor Blades, Including Row-to-Row Interference," Presented at the AIAA $13^{\text {th }}$ Aeroacoustics Conference, Tallahassee, FL, October 22-24. 
Morrison, E., 2013. Development of a Three Dimensional Compressible Flow Calibration Facility for Thermal Anemometry, MS Thesis, Purdue University, West Lafayette, IN.

Richards, S. K., Ramakrishnan, K., Shieh, C., 2012. "Unsteady Acoustic Forcing on an Impeller Due to Coupled Blade Row Interactions," ASME Journal of Turbomachinery, 134, 061014-1 - 061014-9.

Rodgers, C., 1988. "Effect of Inlet Geometry on the Performance of Small Centrifugal Compressors," AIAA/ASME/SAE/ASEE 24th Joint Propulsion Conference, Boston, MA, July 11-13.

Salontay, J. R., Key, N. L., Fulayter, R. D., 2011. “Investigation of Flow Physics of Vane Clocking Effects on Rotor Resonant Response," AIAA Journal of Propulsion and Power, 27-5, 1001-1007.

Schoenenborn, H., Breuer, T., 2012. “Aeroelasticity at Reversed Flow Conditions—Part II: Application to Compressor Surge," ASME Journal of Turbomachinery, 134, 061031-1 061031-8.

Srinivasan, A. V., 1997. "Flutter and Resonant Vibration Characteristics of Engine Blades," ASME Journal of Turbomachinery, 119, 742-771.

Swagelok Technical Sizing Bulletin, 2007, MS-06-84-E. 
Whitehead, D.S., 1987. "Unsteady Two-Dimensional Linearized Subsonic Flow in Cascades." AGARD Manual on Aeroelasticity in Axial-Flow Turbomachines, 1, 3-24 - 330.

Zemp, A., Kammerer, A., Abhari, R. S., 2010. "Unsteady Computational Fluid Dynamics Investigation on Inlet Distortion in a Centrifugal Compressor," ASME Journal of Turbomachinery, 132, 031015-1 - 031015-9.

Zhai, Y., Bladh, R., Dyverfeldt., G., 2012. "Aeroelastic Stability Assessment of an Industrial Compressor Blade Including Mistuning Effects," ASME Journal of Turbomachinery, 134, 060903-1 - 060903-12. 
APPENDICES 


\section{Appendix A Blisk Scan Data}

This section of the appendix will show images of the blisk scans taken in situ by Aerodyne with a portable laser scanner in Figure 6.1 through Figure 6.6. The results show relative mistuning by displaying displacement from nominal 3D CAD geometry using a best fit computer algorithm, where the 3D CAD geometry is fit to the laser scan with the resultant least overall deviation from nominal geometry. The deviations shown are measured perpendicular to the surface in millimeters. Two views, aft facing and forward facing, are shown for each rotor assembly.
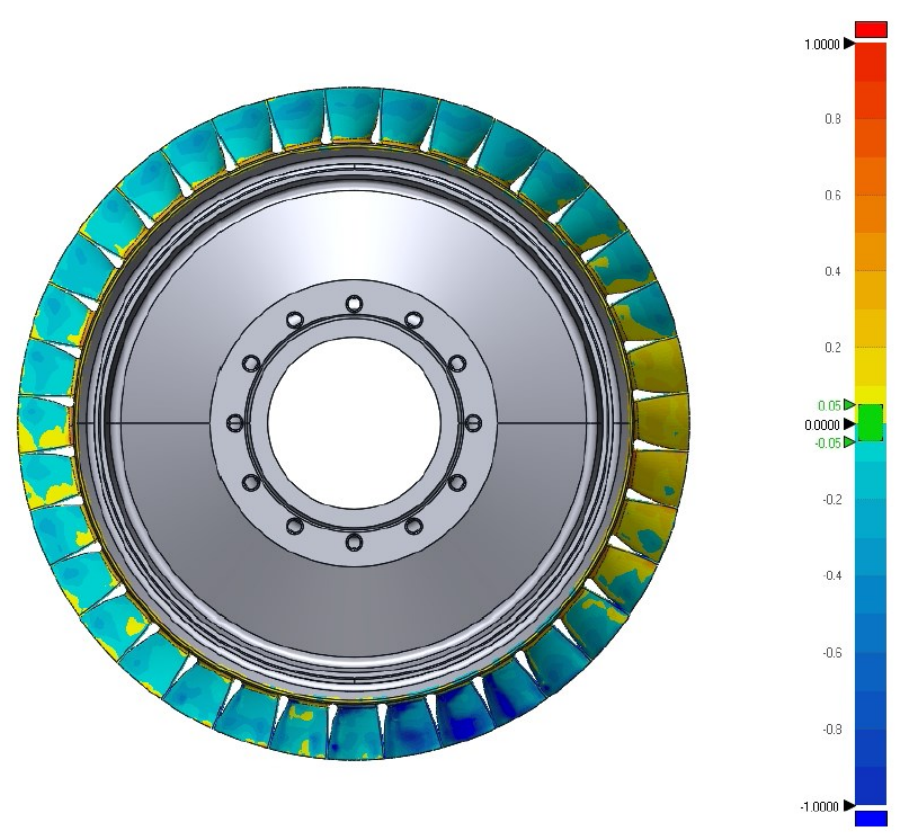

Figure 6.1: Rotor 1 forward looking aft total displacement from nominal, mm. 

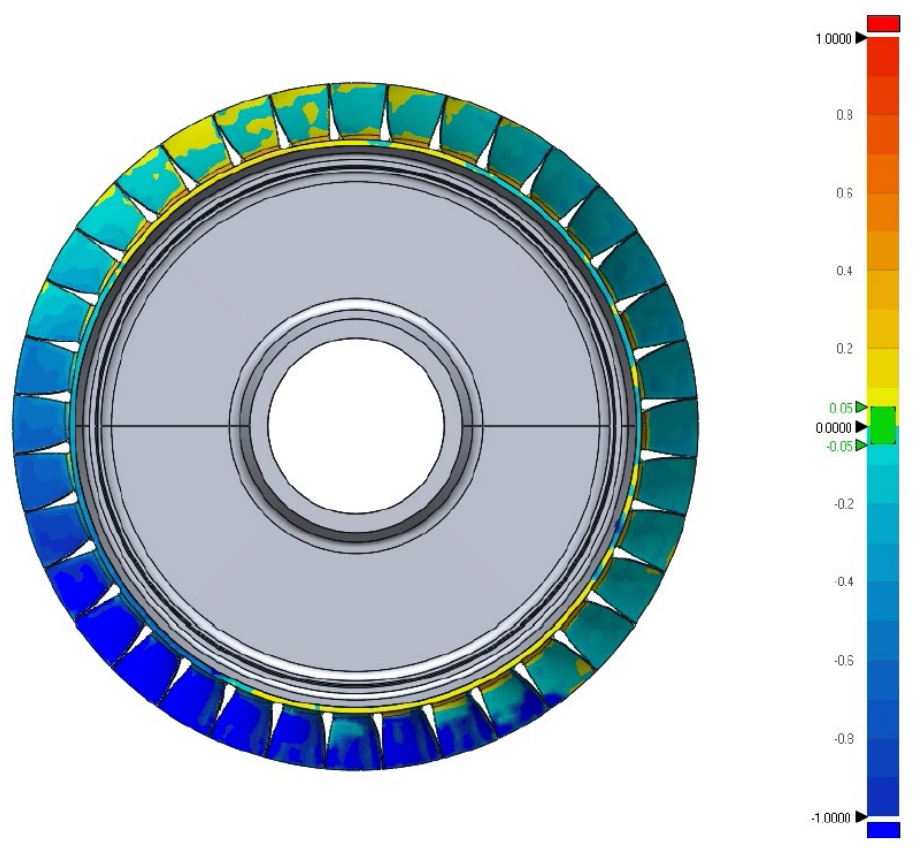

Figure 6.2: Rotor 1 Aft looking forward total displacement from nominal, mm.
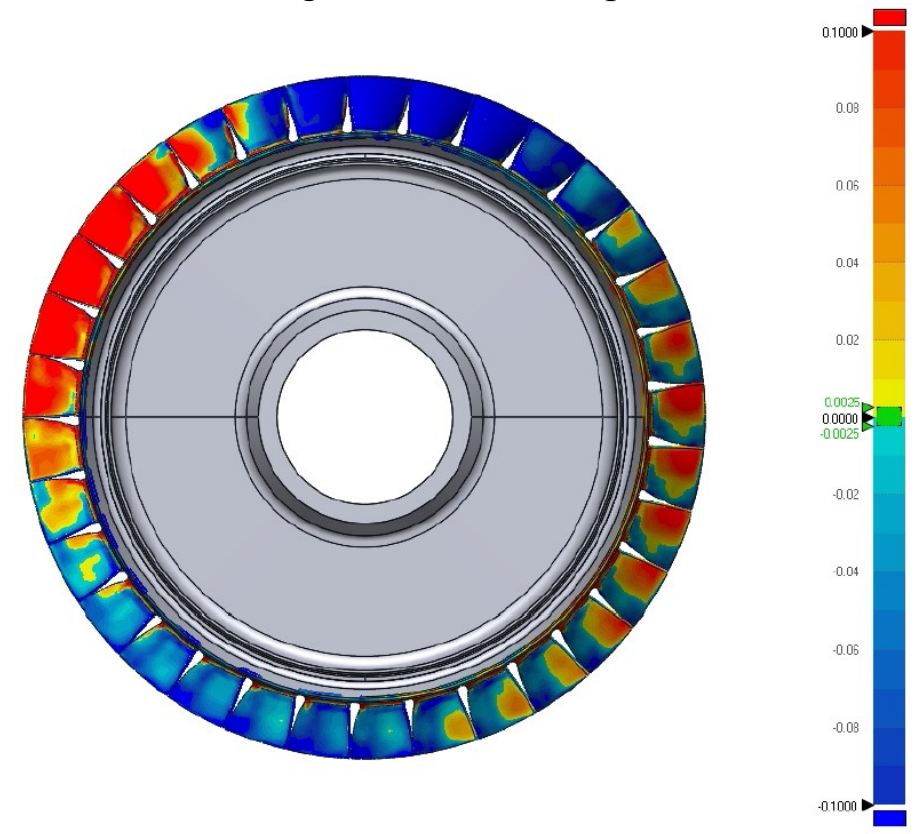

Figure 6.3: R2 forward looking aft total displacement from nominal, mm. 

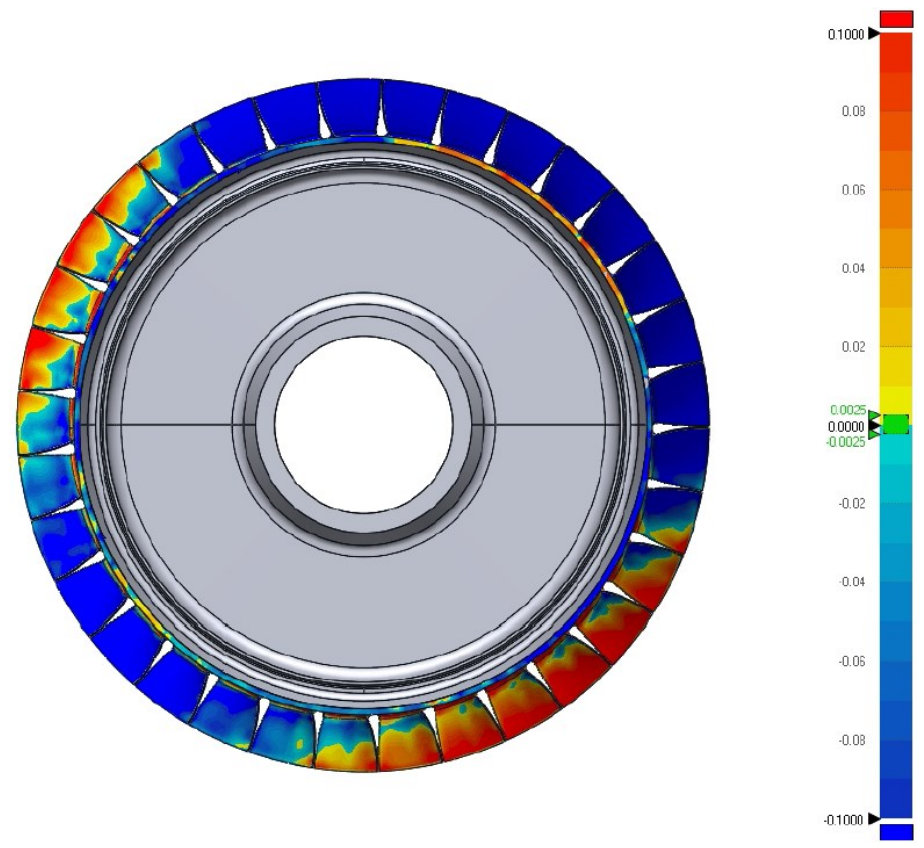

Figure 6.4: R2 Aft looking forward total displacement from nominal, mm.
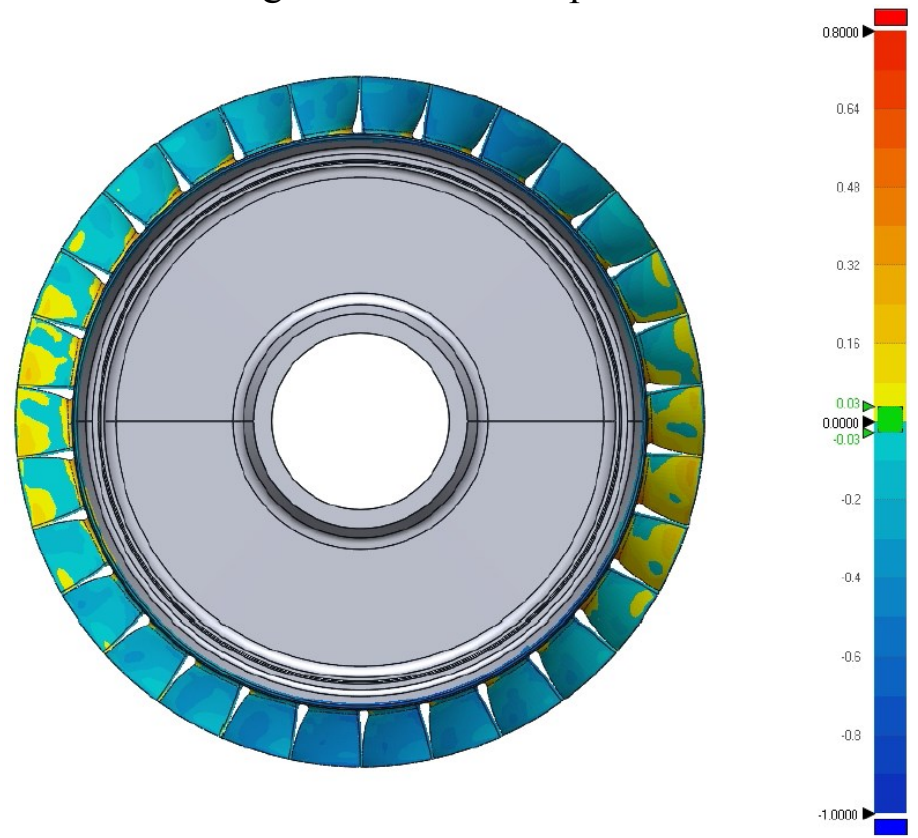

Figure 6.5: R3 Forward looking aft total displacement from nominal, mm. 

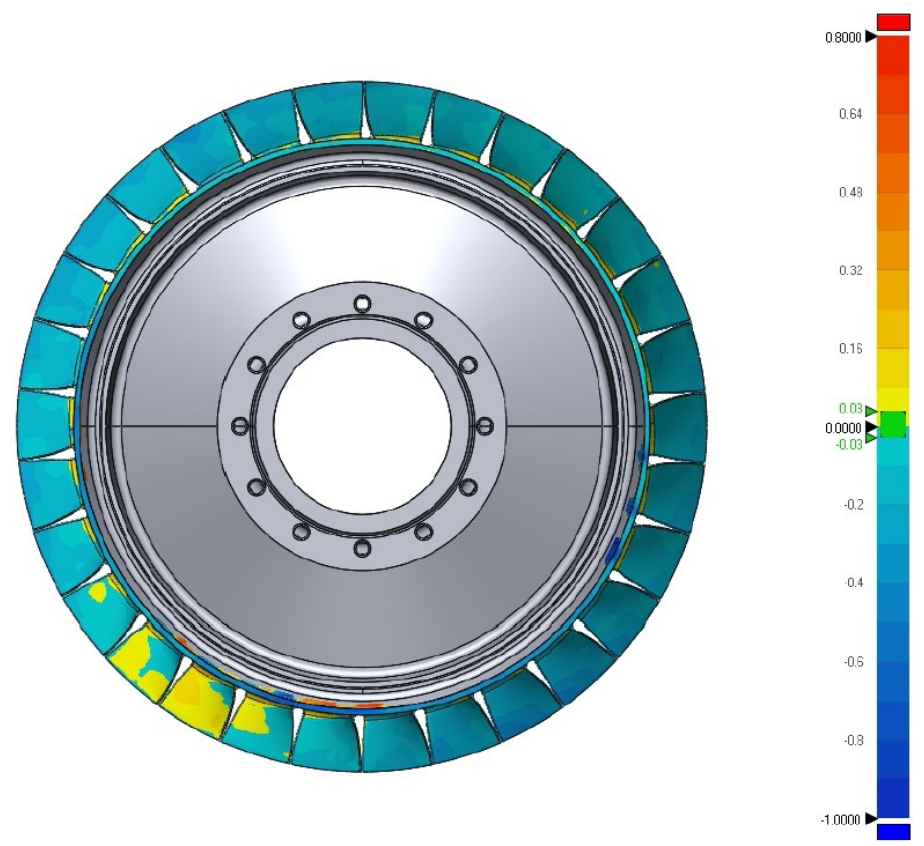

Figure 6.6: R3 Aft looking forward total displacement from nominal, mm. 


\section{Appendix B 3D Hotwire Anemometry}

Throughout the course of testing in this experiment, a total of 3 hotwire probes were used, two of which were 3-wire Dantec hotwire probes, and the other being a TSI crosswire hot film probe. Significant issues were encountered during the testing and data acquisition of the 3D probes, and they will be discussed first.

The first wire tested was a Dantec Probe Type 55 "Crossflow" sensor, similar in prong design to the probe that was used in testing by Morrison, 2013. This probe was known to have significant prong interference at yaw angles around $-20^{\circ}$, but was determined to still have a large enough range in the yaw direction to make accurate measurements. The probe was calibrated in the Purdue Hotwire Calibration Facility for different combinations of air velocity, pitch angle, and yaw angle, relative to the probe. For the calibration the jet speed was varied from $150 \mathrm{ft} /$ to $450 \mathrm{ft} / \mathrm{s}$ in increments of $50 \mathrm{ft} / \mathrm{s}$, and the probe was cycled through flow angles of $-45^{\circ}$ to $45^{\circ}$ in $5^{\circ}$ increments for both pitch and yaw at each velocity. Jet temperature was held to $100^{\circ} \mathrm{F}$ within $+/-1^{\circ} \mathrm{F}$. The same prong interference with this probe was noticed in the calibration space. Data was taken with this probe at stator 1 exit, but when placed at R2 exit, one of the wires broke while taking data.

The second 3-wire probe, a Dantec Type 55-14677501, was then calibrated. This probe was a custom designed probe meant to avoid the prong interference issues of the previous 3D hotwire probe. 


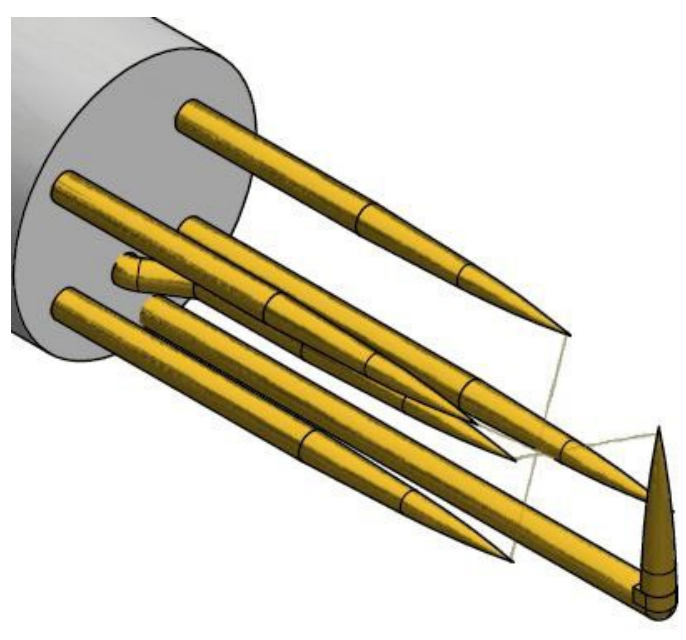

Figure 6.7: Dantec 3D Hotwire Probe Type 55-14677501. Illustration courtesy Dantec Dynamics.

The 3D calibration space, seen in Figure 6.11, has a much different shape than the calibration space for the Crossflow type probe, in

Figure 6.10. It is interesting to note that the span of voltages occupied by the yaw space is much larger than with the previous probe, and by viewing Figure 6.8 , the sensitivity in the positive pitch direction is very high in comparison to the negative pitch direction, especially at high yaw angles. This makes the probe very sensitive to positive pitch measurements, something that is certainly present in the coordinate system of the axial compressor. After testing with this probe, it was evident that the calibration space was calculating very high flow pitch (radial direction) angles. After the testing, the probe was placed back into the calibration device, and air was turned on at $250 \mathrm{ft} / \mathrm{s}$ in order to test the calibration repeatability. It was noted that voltages appeared over $0.1 \mathrm{~V}$ lower than the first calibration, conducted 3 weeks prior, at the same temperature, velocity, and angular positions. Ambient density on the day of testing was known to be higher than on the first day, meaning that if density corrections were applied to the new calibration, it 
would result in an even larger discrepancy in voltage. Before further testing on the probe could be conducted, it was damaged upon unsheathing from the LC Smith positioner that was used to hold the probe, therefore preventing any further testing. It was discovered that a slightly bent probe holder was the culprit - it caused the prongs of the probe that hold the wires to catch on the inside of the device, breaking all three wires and permanently damaging the prongs. It is still not currently known what the source of the non-repeatability in the experimentation was, although one possibility would be that the probe had possibly rubbed a prong against the inside of the LC smith at some point, bending one of the prongs slightly, which could cause the other 5 prongs to loosen as well, affecting the calibration of all three wires. By comparing Table 6-1 to Table 6-2, it is evident that, despite small variations in angle and velocity, the voltages recorded on the later date are all offset to a lower voltage in all cases.

Table 6-1: Select points from calibration of Dantec Type 55-14677501 on 2/21/2013

\begin{tabular}{|c|c|c|c|c|c|c|}
\hline Velocity & Pitch & Yaw & Wire 1 & Wire 2 & Wire 3 & Density \\
\hline$[\mathrm{ft} / \mathrm{s}]$ & {$[\mathrm{deg}]$} & {$[\mathrm{deg}]$} & {$[\mathrm{V}]$} & {$[\mathrm{V}]$} & {$[\mathrm{V}]$} & {$\left[\mathrm{lbm} / \mathrm{ft}^{3}\right]$} \\
\hline 201.6 & -30.0 & -41.9 & 3.682 & 3.528 & 3.452 & 0.070 \\
\hline 199.0 & -30.0 & -25.0 & 3.684 & 3.599 & 3.280 & 0.069 \\
\hline 197.9 & -30.0 & -19.7 & 3.689 & 3.640 & 3.319 & 0.070 \\
\hline 199.8 & -0.3 & 0.1 & 3.560 & 3.578 & 3.558 & 0.070 \\
\hline 198.0 & -0.3 & 5.0 & 3.527 & 3.616 & 3.563 & 0.070 \\
\hline 197.9 & -0.3 & 10.1 & 3.441 & 3.638 & 3.555 & 0.070 \\
\hline
\end{tabular}


Table 6-2: Select points from calibration of Dantec Type 55-1467750 on 3/10/2013

\begin{tabular}{|c|c|c|c|c|c|c|}
\hline Velocity & Pitch & Yaw & Wire 1 & Wire 2 & Wire 3 & Density \\
\hline$[\mathrm{ft} / \mathrm{s}]$ & {$[\mathrm{deg}]$} & {$[\mathrm{deg}]$} & {$[\mathrm{V}]$} & {$[\mathrm{V}]$} & {$[\mathrm{V}]$} & {$\left[\mathrm{lbm} / \mathrm{ft}^{3}\right]$} \\
\hline 204.9 & -30.0 & -40.0 & 3.609 & 3.470 & 3.464 & 0.070 \\
\hline 202.7 & -30.0 & -24.9 & 3.608 & 3.498 & 3.348 & 0.070 \\
\hline 200.8 & -30.0 & -20.0 & 3.609 & 3.526 & 3.275 & 0.070 \\
\hline 201.6 & 0.1 & 0.0 & 3.456 & 3.492 & 3.553 & 0.070 \\
\hline 198.8 & 0.1 & 4.9 & 3.437 & 3.511 & 3.567 & 0.070 \\
\hline 199.5 & 0.1 & 10.1 & 3.340 & 3.545 & 3.547 & 0.070 \\
\hline
\end{tabular}

Both Dantec probes were connected to a Dantec Dynamics StreamWare Pro chassis with a 91C10 anemometry module for each of the three channels. The voltages were recorded with a NI PXI-1073 chassis with a NI-PXIE-6358 card, and read into LabView. Data was taken at $100 \mathrm{kHz}$ for each channel, and for each point in the vane passage, 8.5 seconds of data was recorded, which, at speeds around $74 \% \mathrm{~N}_{\mathrm{c}}$, corresponds to over 500 revolutions of data.

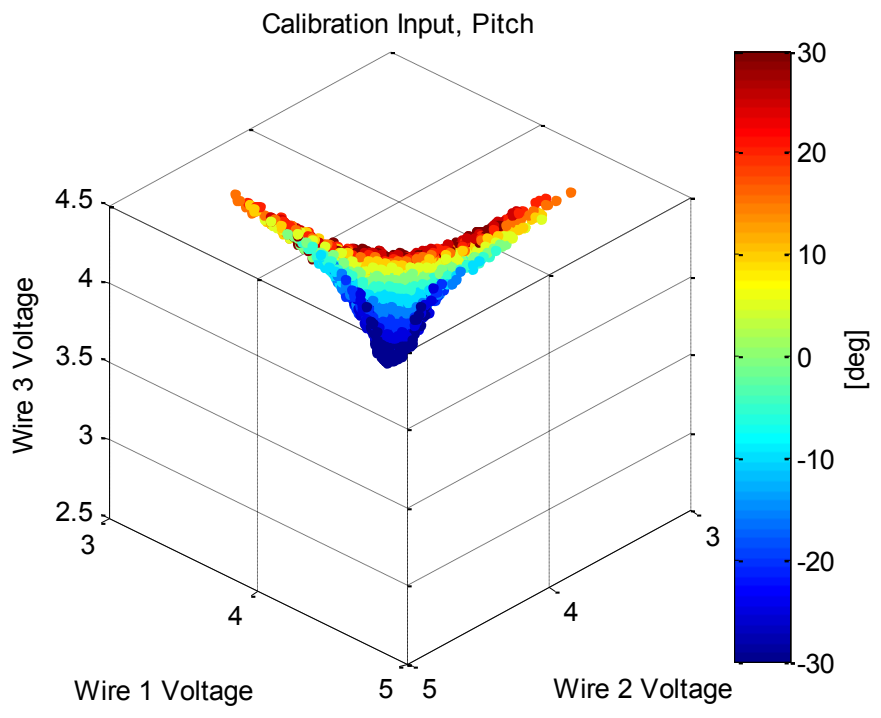

Figure 6.8: View of 3D calibration space in which pitch wise voltage variation is illustrated for Dantec Probe Type 55-14677501. 

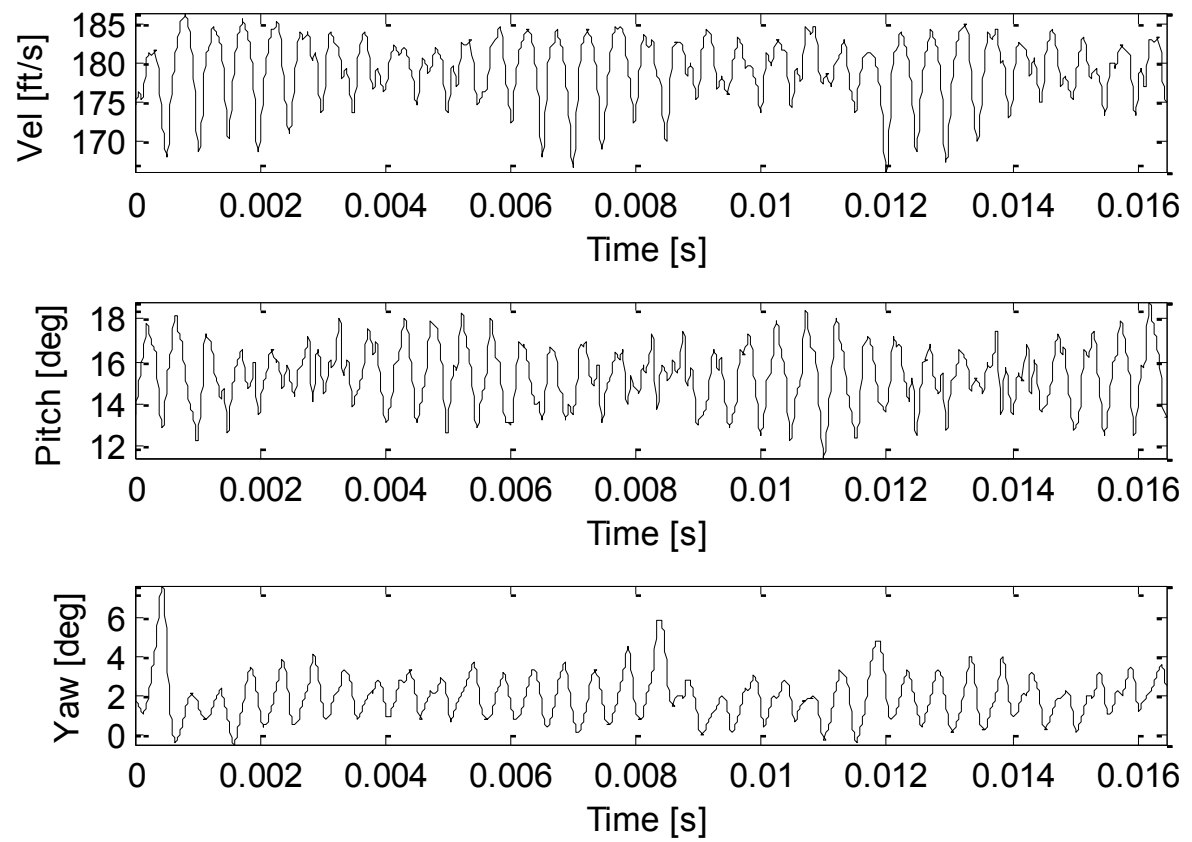

Figure 6.9: EA S1 exit data away from vane wake, NL, 50\% Span, showing unnaturally high pitch angles as a result of calibration drift in hardware. No temp or density corrections. 

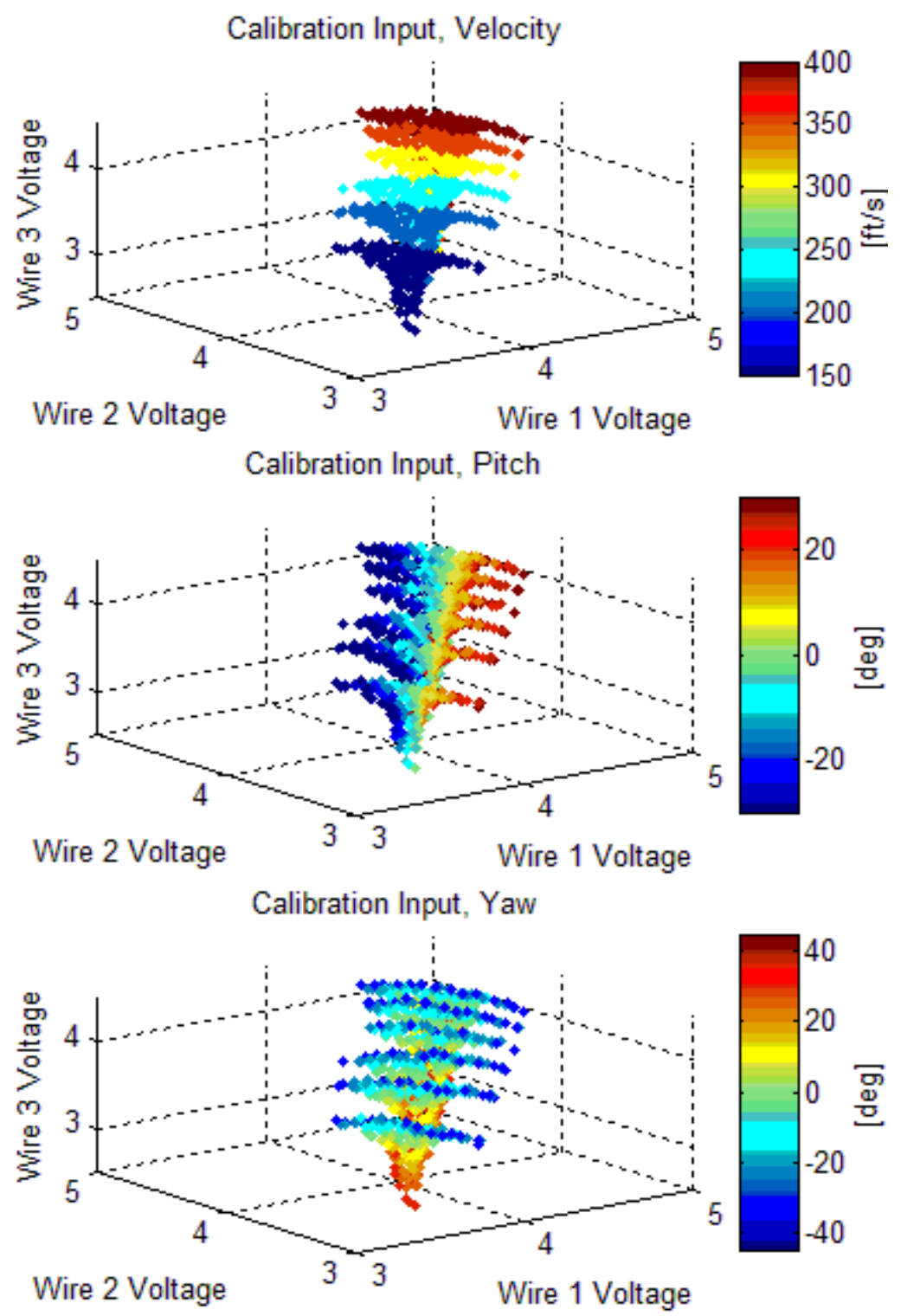

Figure 6.10: 3D Calibration space of Dantec Type 55-Crossflow probe. 

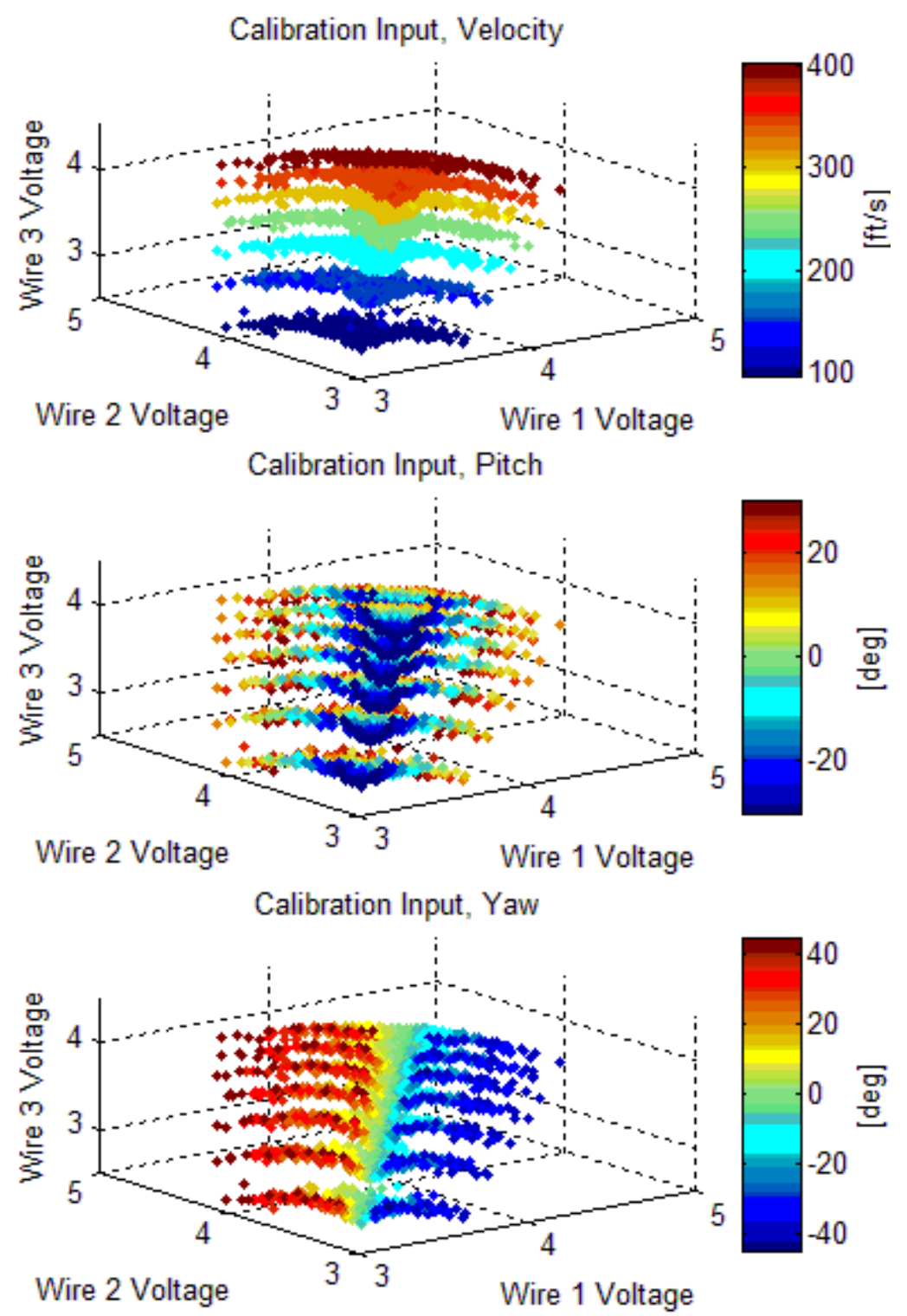

Figure 6.11: 3D Calibration space of Dantec Type 55-14677501 probe. 


\section{Appendix C Kulite Windowing Techniques}

This section will display sample images displaying a few variations on the windowing techniques investigated to display changing frequency spectra. Figure 6.12 shows the normalized windowing coefficient for the three windowing functions investigated, showing the weakest window being a Kaiser window and Hamming window as the strongest.

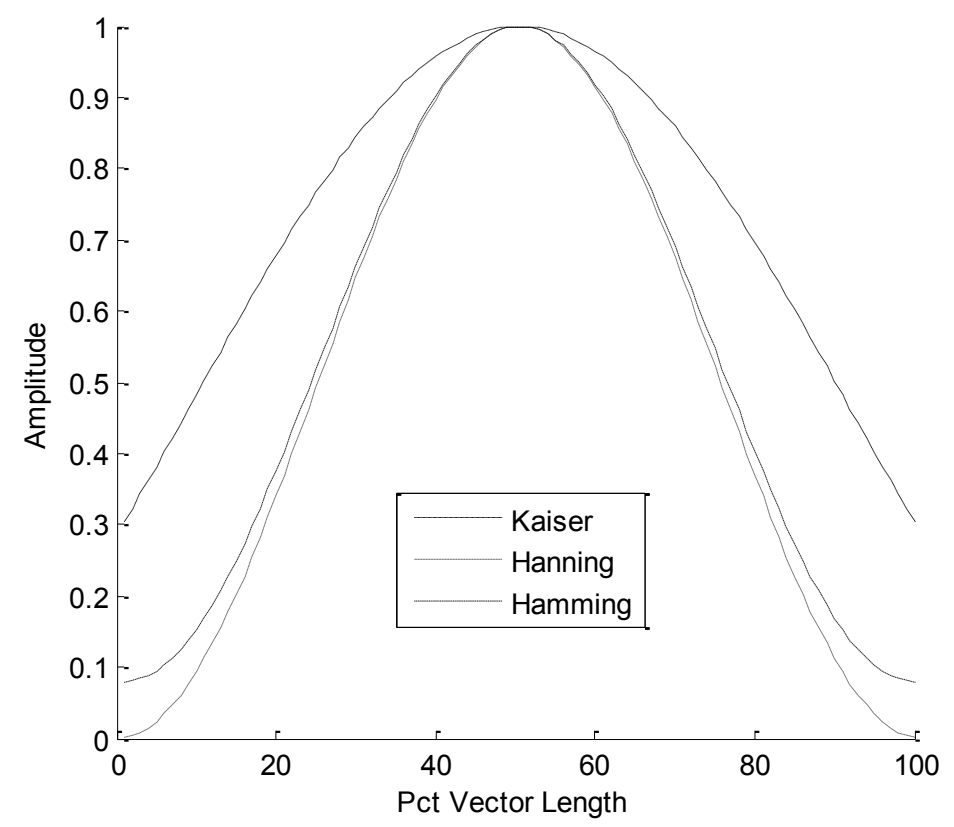

Figure 6.12: Comparison of Kaiser, Hanning, and Hamming windows.

Figure 6.13 shows data from a randomly selected Kulite - in this case, Stator Kulite at $80 \%$ span, $40 \%$ chord at NL with a very large range of frequency shown. Figure 6.14 is shows the same data as Figure 6.13 however it is a zoomed in image in the frequency domain, focusing on the first 3 harmonics of R2 BPF $(30,60$, and 90). 


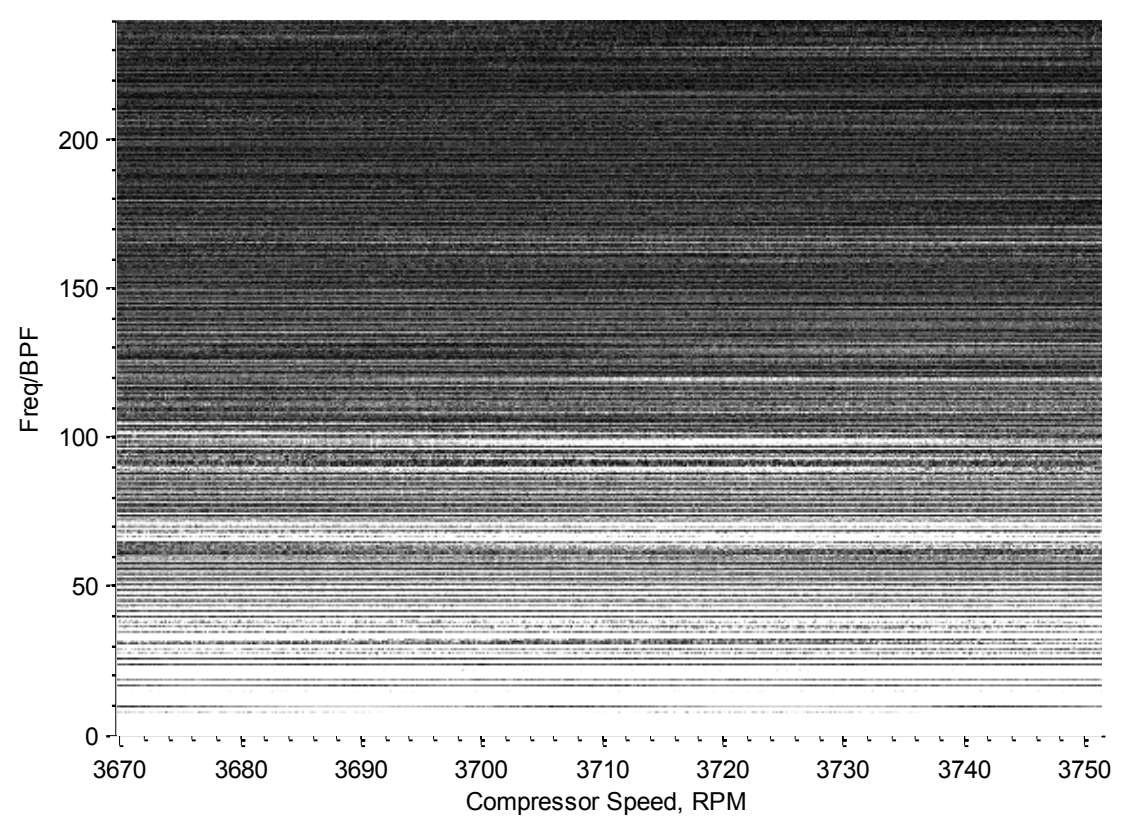

Figure 6.13: Stator Kulite 80\% Span 40\% Chord, no windowing, large range.

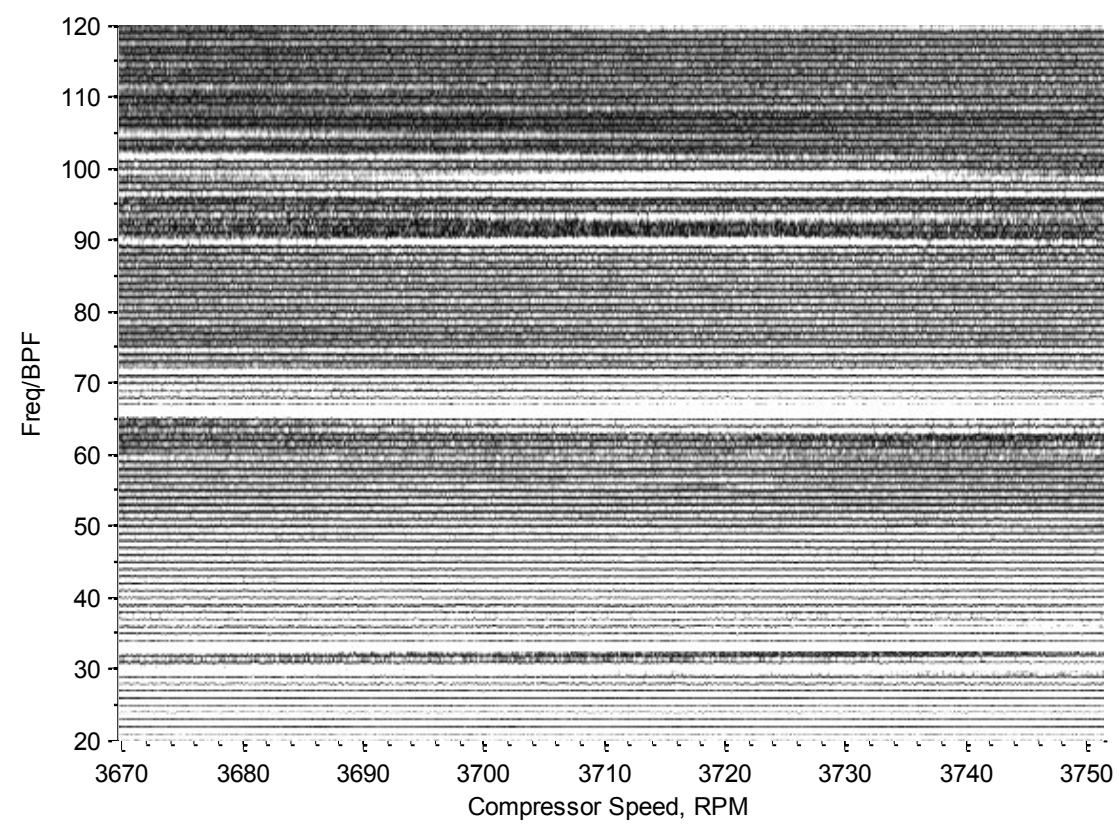

Figure 6.14: Same data as Figure 6.13, reduced range, no windowing.

As discussed in previous sections, all Fourier waterfall plots are shown after a subtraction of steady BPF spectra has been undertaken. Figure 6.15, Figure 6.16, and 
Figure 6.17 show a Kaiser, Hanning, and Hamming window, resepectively, applied to

Figure 6.14. These are all shown without subtracting steady, non-vibrating spectra.

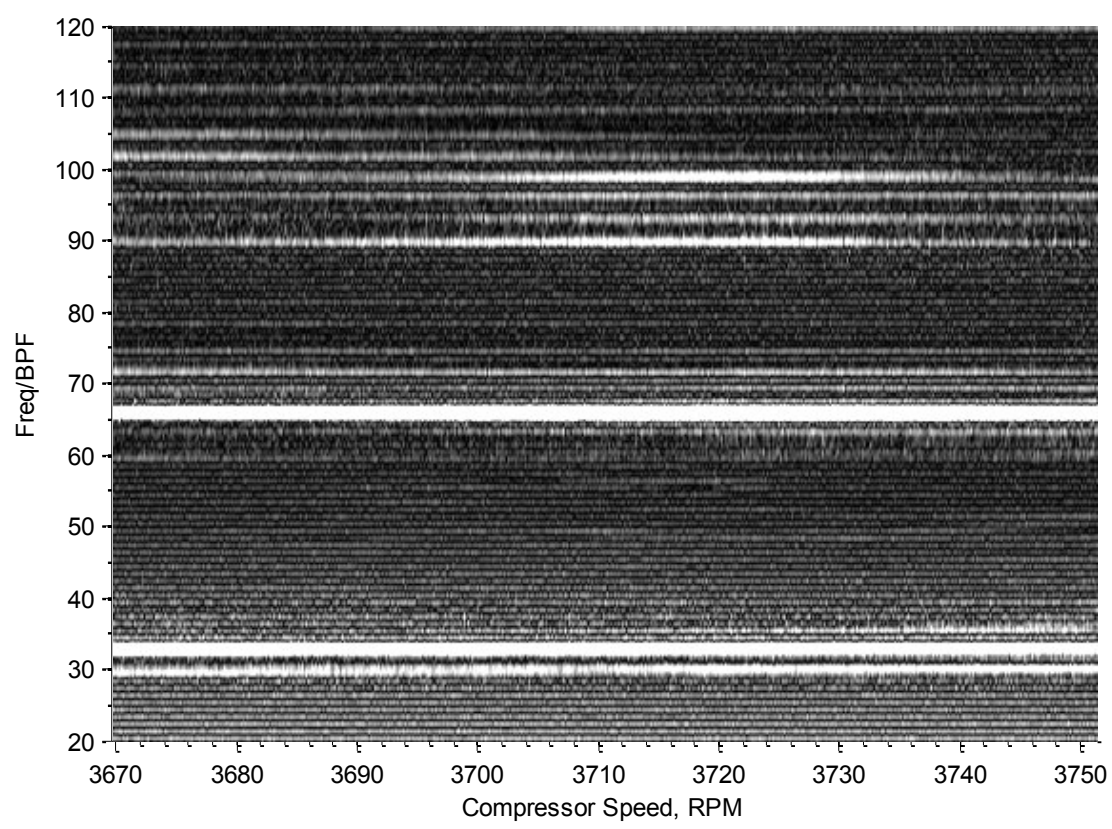

Figure 6.15: Same data as Figure 6.14, Kaiser windowing.

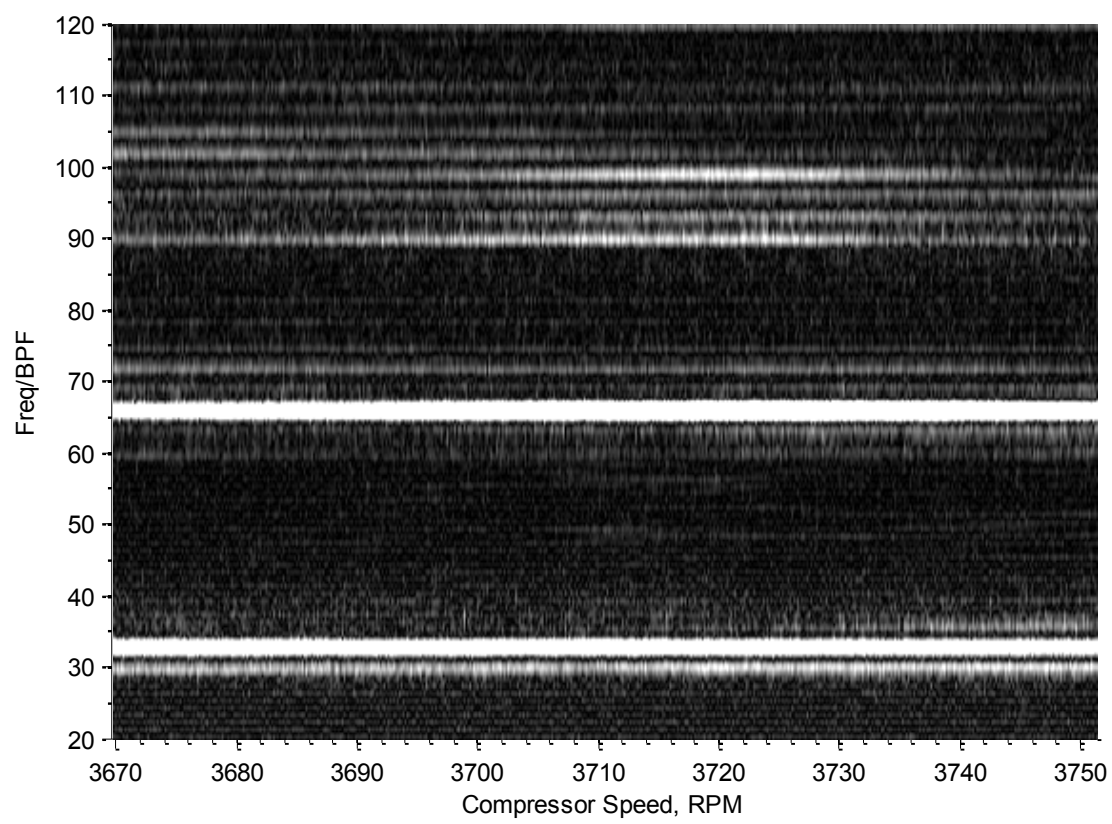

Figure 6.16: Same data as Figure 6.14, Hamming windowing. 


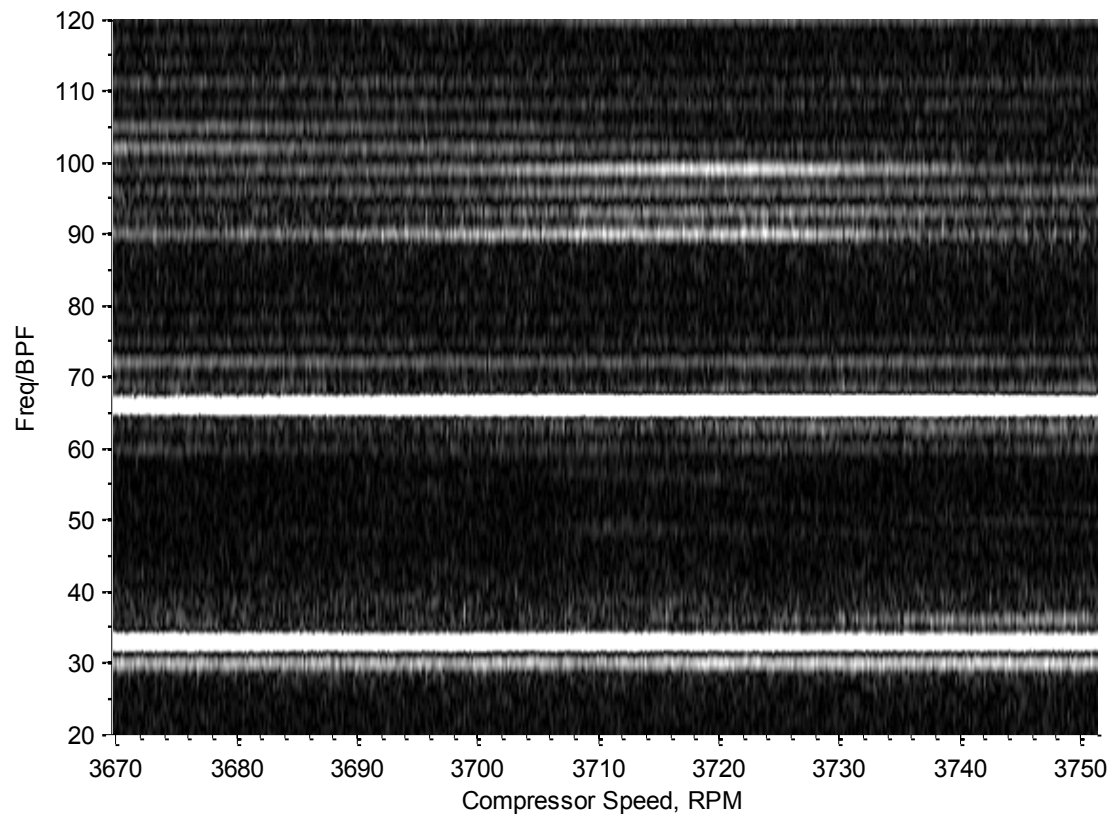

Figure 6.17: Same data as Figure 6.14, Hanning windowing.

Figure 6.18 shows the plot after steady spectra subtraction at 3670 RPM.

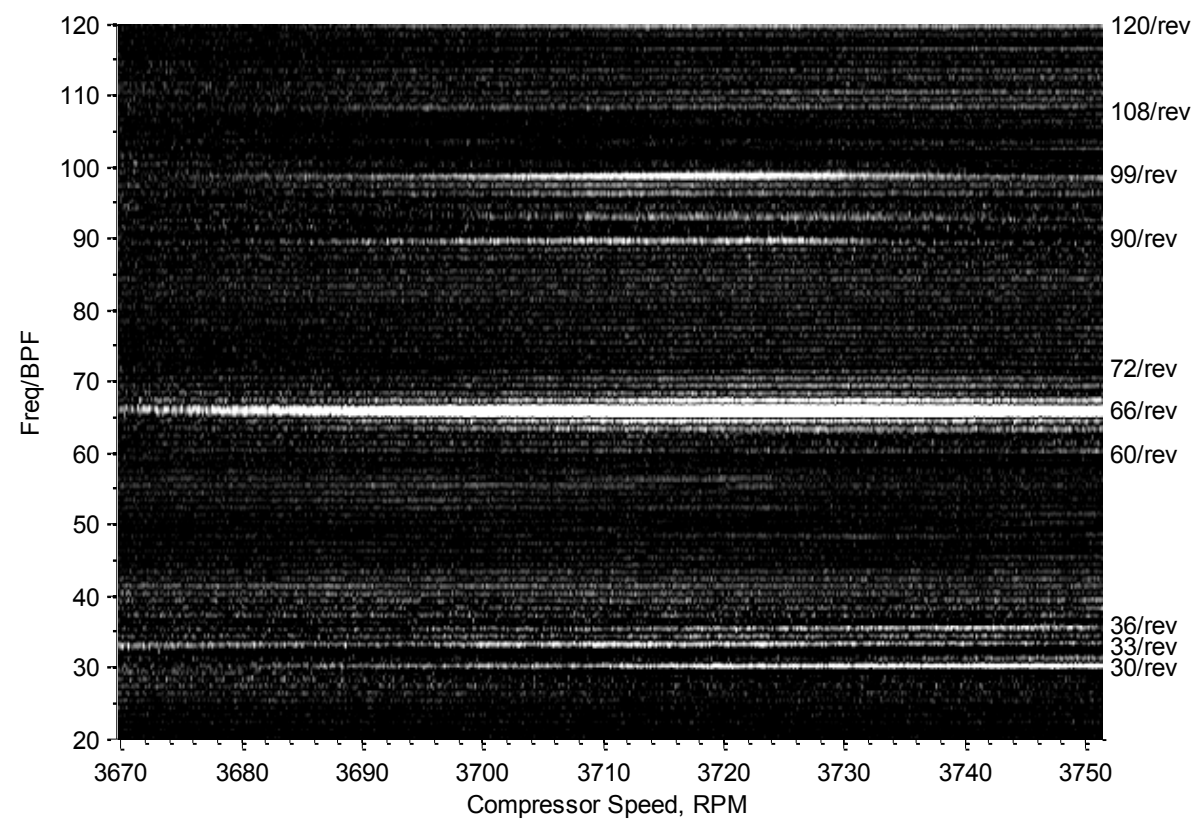

Figure 6.18: Same data as Figure 6.15, subtract out steady spectra, Kaiser windowing. 


\section{Appendix D Vibrometry Data}

This section of the appendix will show, in Figure 6.19 through Figure 6.25 the results of stator vibrometry testing conducted at Wright Patterson Air Force Base in Dayton, $\mathrm{OH}$. Each plot is of one stator section. The stator sections tested were one full shrouded half ring from all stages - IGV, S1, S2, and S3. Additionally, there were three other stator sections tested, all from the stator assembly that was used in testing the Kulite instrumented section. These are grouped in Figure 6.21 through Figure 6.23 and labeled as "small", "medium" and "large", per Figure 3.6. Each line on the plot corresponds to the response measured by one blade, and all blades from all sections are overlaid on each plot to give an idea of the overall envelope of response for each stator section tested. Amplitudes are measured in mils.

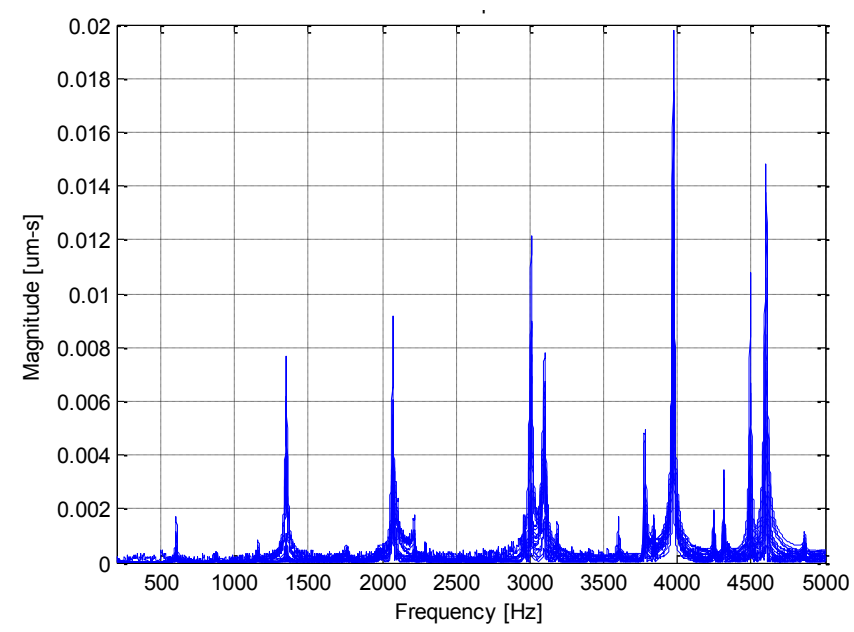

Figure 6.19: IGV Vibrometry response 


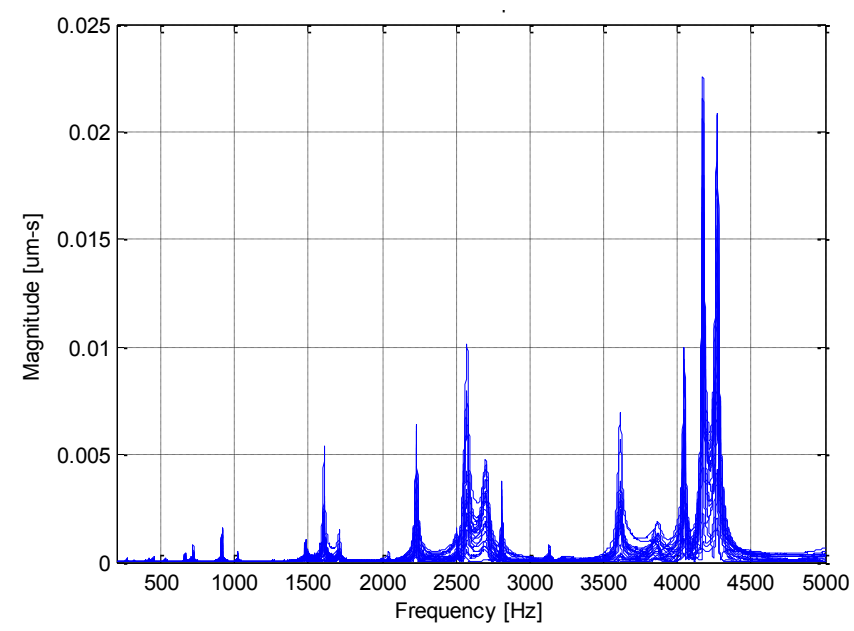

Figure 6.20: Stator 1 Vibrometry response.

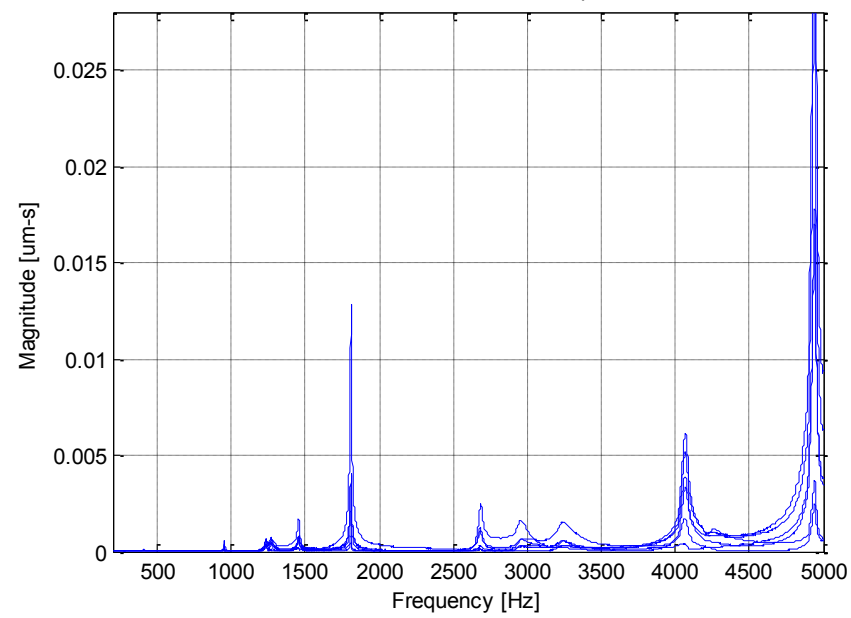

Figure 6.21: Stator 2 Medium Section Vibrometry response. 


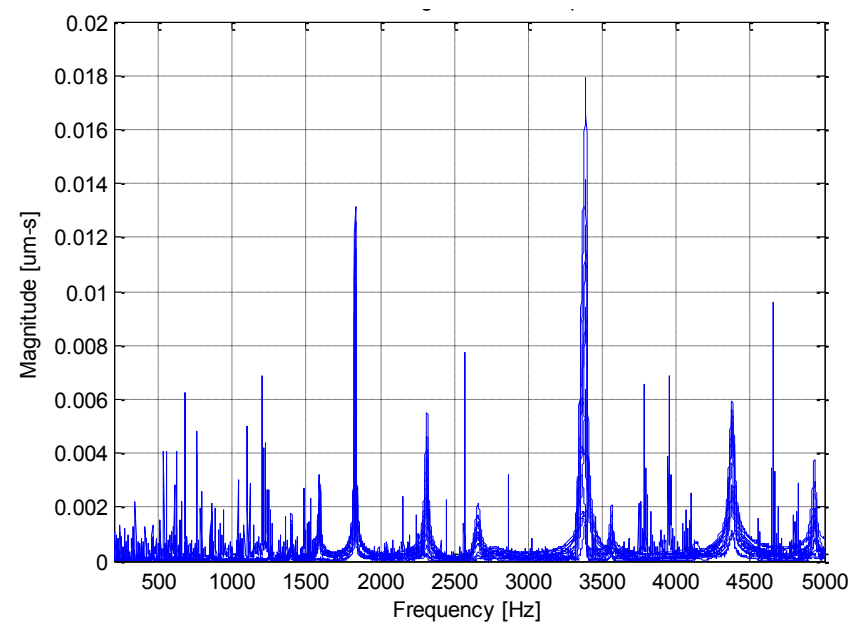

Figure 6.22: Stator 2 Large Section Response.

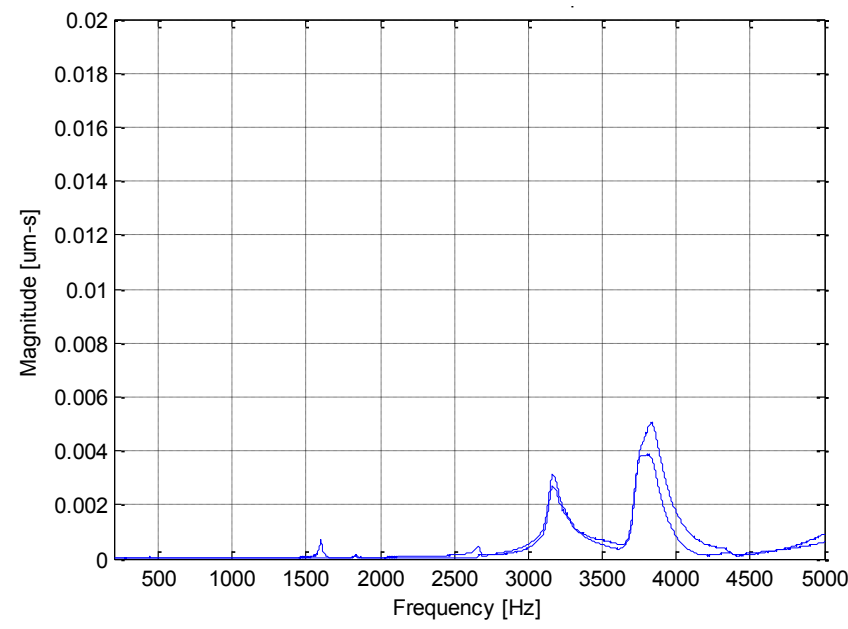

Figure 6.23: Stator 2 Small Section Response. 


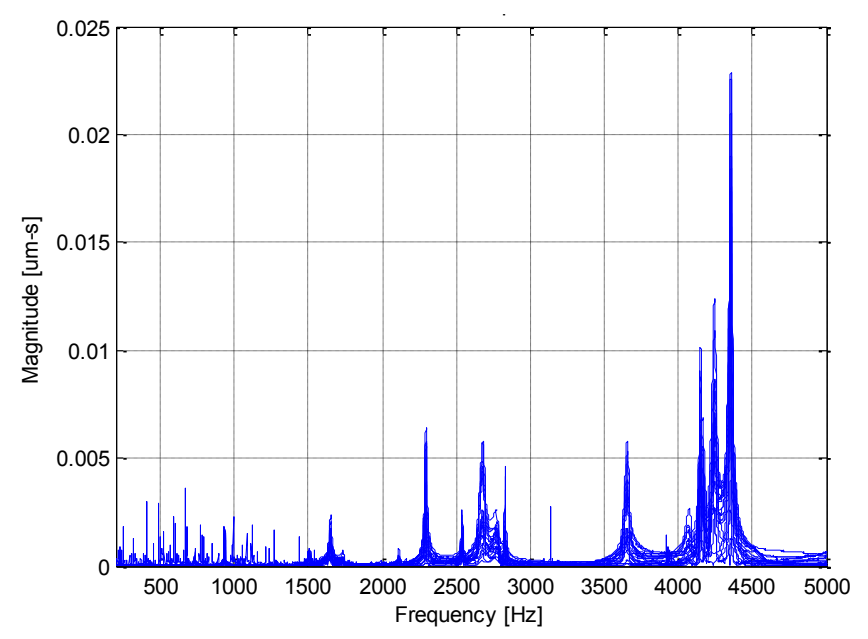

Figure 6.24: Stator 2 full half-ring response.

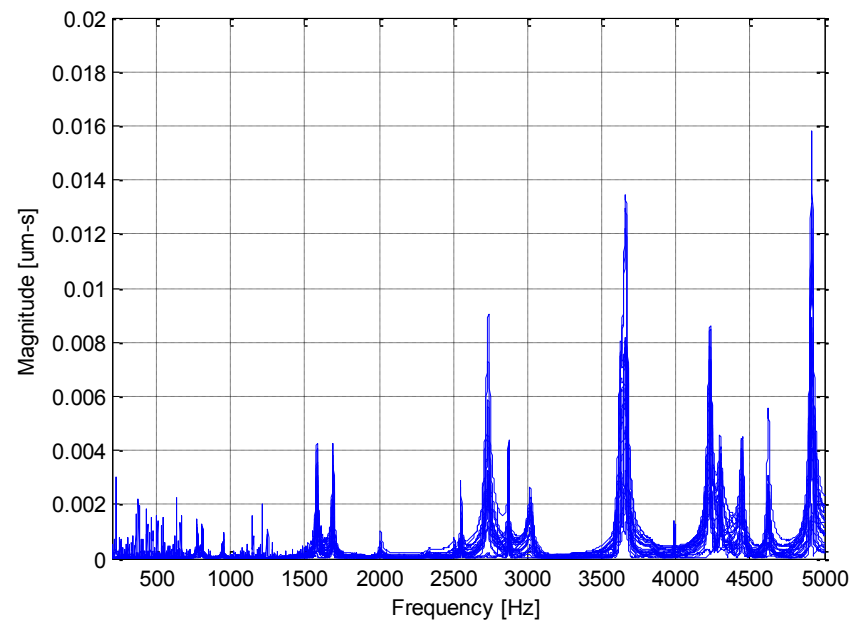

Figure 6.25: Stator 3 half-ring response. 


\section{Appendix E Error Summary}

Uncertainties in NSMS data are calculated by assuming that each blade constitutes a damped single degree of freedom mass spring damper system, and fitting phase, amplitude, and frequency data from each blade to the system using a least-squares fit. This is the principle behind what Agilis calls the Least-Squares Model Fit (LSMF) calculation module from which all deflections and damping coefficients are calculated. Eqn. 6-1 shows how the uncertainty in blade deflection is calculated. Data fit is calculated from how well the least-squares model is able to fit the data gathered.

$$
\text { Uncertaint }_{P-P}=(100-\text { data fit }) * P-P_{a m p} \quad \text { Eqn. 6-1 }
$$

Table 6-3 shows the calculated P-P deflection and corresponding Data Fit value from each blade for all 4 data runs presented in this thesis.

Table 6-3: NSMS P-P deflection uncertainty and LSMF model fit number per R2 blade \#.

\begin{tabular}{|c|c|c|c|c|c|c|c|c|}
\hline & $\begin{array}{c}\text { NL } \\
\text { Accel }\end{array}$ & & $\begin{array}{c}\text { NL } \\
\text { Decel }\end{array}$ & & $\begin{array}{c}\text { HL } \\
\text { Accel }\end{array}$ & & $\begin{array}{c}\text { HL } \\
\text { Decel }\end{array}$ & \\
\hline \# & $\begin{array}{c}+/- \\
{[\mathrm{mils}]}\end{array}$ & $\begin{array}{c}\text { Data } \\
\text { Fit }\end{array}$ & $+/-[\mathrm{mils}]$ & $\begin{array}{c}\text { Data } \\
\text { Fit }\end{array}$ & $+/-[\mathrm{mils}]$ & $\begin{array}{l}\text { Data } \\
\text { Fit }\end{array}$ & $+/-[\mathrm{mils}]$ & $\begin{array}{c}\text { Data } \\
\text { Fit }\end{array}$ \\
\hline 1 & 0.076 & 98.62 & 0.318 & 95.58 & 0.073 & 99.35 & 0.327 & 97.37 \\
\hline 2 & 0.435 & 94.62 & 0.139 & 98.46 & 0.236 & 98.07 & 0.420 & 97.00 \\
\hline 3 & 0.328 & 96.16 & 0.236 & 97.88 & 0.290 & 97.59 & 0.551 & 96.00 \\
\hline 4 & 0.296 & 96.27 & 0.631 & 94.50 & 0.333 & 96.85 & 0.821 & 94.42 \\
\hline 5 & 0.326 & 94.67 & 0.841 & 86.19 & 0.462 & 95.78 & 0.387 & 97.08 \\
\hline 6 & 0.179 & 96.15 & 0.231 & 93.22 & 0.394 & 95.56 & 0.153 & 97.78 \\
\hline 7 & 0.418 & 92.63 & 0.383 & 90.06 & 0.372 & 95.41 & 0.609 & 91.66 \\
\hline 8 & 0.134 & 96.19 & 0.186 & 97.32 & 0.912 & 93.51 & 0.319 & 97.48 \\
\hline 9 & 0.424 & 94.61 & 0.621 & 93.61 & 0.194 & 98.79 & 0.239 & 98.75 \\
\hline 10 & 0.413 & 92.34 & 0.938 & 81.52 & 1.849 & 89.31 & 1.967 & 89.50 \\
\hline 11 & 0.439 & 90.30 & 1.386 & 71.14 & 0.474 & 97.15 & 0.281 & 98.42 \\
\hline
\end{tabular}




\begin{tabular}{|c|c|c|c|c|c|c|c|c|}
\hline 12 & 0.093 & 98.02 & 0.105 & 98.78 & 0.108 & 98.41 & 0.140 & 98.80 \\
\hline 13 & 0.188 & 95.57 & 0.550 & 92.59 & 0.132 & 96.94 & 0.230 & 96.55 \\
\hline 14 & 1.295 & 86.39 & 0.687 & 90.68 & 1.270 & 88.48 & 0.780 & 92.88 \\
\hline 15 & 0.300 & 96.37 & 0.261 & 95.51 & 0.629 & 95.59 & 0.132 & 98.77 \\
\hline 16 & 1.176 & 88.33 & 1.237 & 90.28 & 0.455 & 97.87 & 0.605 & 97.08 \\
\hline 17 & 0.324 & 94.63 & 1.065 & 86.00 & 0.455 & 96.66 & 1.193 & 91.49 \\
\hline 18 & 0.343 & 92.46 & 0.380 & 92.70 & 0.887 & 92.64 & 0.559 & 95.60 \\
\hline 19 & 0.239 & 95.70 & 0.091 & 98.54 & 0.102 & 99.29 & 0.364 & 97.77 \\
\hline 20 & 0.173 & 91.47 & 0.363 & 86.10 & 0.240 & 95.49 & 0.300 & 95.30 \\
\hline 21 & 0.961 & 80.07 & 2.321 & 62.42 & 0.215 & 98.80 & 1.493 & 91.69 \\
\hline 22 & 0.256 & 96.53 & 0.320 & 94.51 & 0.057 & 99.62 & 0.271 & 98.22 \\
\hline 23 & 0.118 & 99.16 & 0.466 & 96.32 & 0.352 & 98.28 & 0.493 & 97.46 \\
\hline 24 & 1.132 & 91.01 & 1.381 & 87.50 & 1.935 & 85.91 & 3.078 & 74.52 \\
\hline 25 & 1.998 & 76.00 & 1.552 & 74.96 & 0.893 & 92.71 & 2.162 & 84.27 \\
\hline 26 & 0.528 & 96.80 & 0.407 & 97.74 & 0.761 & 97.08 & 1.436 & 95.25 \\
\hline 27 & 0.189 & 97.70 & 0.639 & 92.16 & 0.508 & 96.55 & 1.074 & 94.14 \\
\hline 28 & 0.561 & 92.88 & 0.302 & 95.66 & 0.471 & 96.88 & 0.727 & 95.16 \\
\hline 29 & 1.271 & 89.53 & 1.174 & 89.22 & 0.398 & 98.17 & 0.382 & 98.07 \\
\hline 30 & 0.741 & 90.71 & 0.961 & 86.92 & 0.453 & 97.21 & 0.391 & 98.24 \\
\hline 31 & 0.401 & 96.39 & 0.993 & 87.58 & 0.981 & 95.77 & 1.399 & 94.84 \\
\hline 32 & 0.690 & 86.56 & 0.303 & 93.91 & 0.820 & 95.11 & 0.558 & 95.55 \\
\hline 33 & 0.335 & 90.90 & 0.503 & 85.69 & 0.610 & 90.66 & 0.897 & 85.91 \\
\hline Avg & 0.508 & 92.90 & 0.665 & 90.17 & 0.550 & 95.80 & 0.751 & 94.63 \\
\hline Min & 0.076 & 76.00 & 0.091 & 62.42 & 0.057 & 85.91 & 0.132 & 74.52 \\
\hline Max & 1.998 & 99.16 & 2.321 & 98.78 & 1.935 & 99.62 & 3.078 & 98.80 \\
\hline
\end{tabular}

Kulite XTL-140 and LQ-062 pressure transducers were used to measure all fastresponse pressures. Kulite lists the combined non-linearity, hysteresis and repeatability as $+/-0.1 \%$ to $+/-0.5 \%$ of the full scale range for each transducer, which is $0-5 p s i g$. The transducer uncertainty is therefore up to $+/-.025$ psig.

The Precision Filter 28118 amplifier cards have an accuracy of $+/-0.1 \%$ of full scale range, which is $10 \mathrm{~V}$ for the Kulite output with Gain $=100$, which was used for all testing. This amounts to $+/-0.01 \mathrm{~V}$, which is an additional $+/-.005$ psig of uncertainty added to the Kulites. 
The uncertainty component from the PXI-6358/6356 A/D converters is .012\% FS. Based on a $10 \mathrm{~V}$ input range, this gives an uncertainty of $+/-0.0012 \mathrm{~V}$, which is equal to $+/-$ 0.00006 psig. By simply combining the three sources of error, the resulting accuracy in the Kulite pressure measurements amounts to approximately $+/-0.031 \mathrm{psig}$.

The mass flow rate measurements were made using a long-form ASME standard venturi, where inlet and throat pressure measurements were made with high-accuracy Setra differential pressure transducers. The REFPROP thermodynamic program is used to calculate flow density by appropriately considering humidity effects. The uncertainty in corrected mass flow rate is $+/-1.4 \%$, and $60 \%$ of this uncertainty is due to ASME-standard uncertainty in discharge coefficient. A digital hygrometer measures relative humidity to within $+/-2 \%$. Resulting design point total pressure ratio for stage 2 using these accuracies is $+/-0.00027$.

On inter stage measurements, Scanivalve Digital Sensor Arrays, containing 16 piezo-resistive pressure sensors with an uncertainty of $+/-0.05 \%$ FS were used. The pressure transducers are zeroed every 20 minutes of testing to mitigate temperature drift issues. The Scanivalve arrays were used for all rake and casing pressure measurements. Ambient reference pressure is measured with a Setra high accuracy transducer with an uncertainty of $+/-0.073 \%$ FS.

For compressor temperature measurements, special limits-of-error T-type thermocouples were used, and in conjunction with Agilent 34980A Muliplexer thermocouple modules, the resulting uncertainty is $+/-0.3^{\circ} \mathrm{C}$. Thermal bath calibrations have verified temperature rake measurement repeatability. 
The TSI hotwire probe was calibrated in the Purdue Compressible Flow Calibration Facility, detailed by Morrison (2013). He quotes a temperature variance within the jet flow core of less than $0.5 \%$, and pressure variance of $0.5 \%$. Velocity set points during calibration were set to $+/-3 \mathrm{ft} / \mathrm{s}$ and temperature within $+/-1 \%$, which is a good measure of the stability of each calibration point. The exact temperature and velocity as calculated by Setra pressure transducers, however, was recorded for each point. Morrison (2013) quotes a calibration temperature uncertainty of $+/-0.54^{\circ} \mathrm{C}$. Overall velocity uncertainty has a range from $+/-0.8 \mathrm{ft} / \mathrm{s}$ at minimum pressure, and $+/-4.1 \mathrm{ft} / \mathrm{s}$ at minimum velocity and maximum temperature. Maximum density uncertainty is $5.5 \times 10^{-5} \mathrm{lb} / \mathrm{ft}^{3}$.

ScatteredInterpolant was used as a lookup function for calculating measured velocities and flow angles. The lookup space is highly non-linear although it uses a linear approximation between nearest neighbor points in a $2 \mathrm{D}$ space to calculate calibrated values. Due to non-uniformities in the lookup space, the uncertainty in the lookup process is highly dependent on input values and difficult to quantify. However, a matrix of 10 points were input to the lookup tables where real wire voltages were known, and maximum errors from the lookup process were $5.2 \mathrm{ft} / \mathrm{s}$ in velocity and $1.6^{\circ}$ in alpha. 